\title{
Equilibrium Isotope Effects as a Probe of Nonbonding Attractions
}

\author{
Yi-Lei Zhao, ${ }^{a}$ K. N. Houk, ${ }^{* a}$ Dalit Rechavi, ${ }^{\mathrm{b}}$ Alessandro Scarso, and Julius Rebek, $\mathrm{Jr}^{\mathrm{b}}$
}

\section{SUPPORTING INFORMATION}

All structures discussed are computed with Gaussian 03 programs (Revision B.05) by:

M. J. Frisch, G. W. Trucks, H. B. Schlegel, G. E. Scuseria,

M. A. Robb, J. R. Cheeseman, J. A. Montgomery, Jr., T. Vreven,

K. N. Kudin, J. C. Burant, J. M. Millam, S. S. Iyengar, J. Tomasi,

V. Barone, B. Mennucci, M. Cossi, G. Scalmani, N. Rega,

G. A. Petersson, H. Nakatsuji, M. Hada, M. Ehara, K. Toyota,

R. Fukuda, J. Hasegawa, M. Ishida, T. Nakajima, Y. Honda, O. Kitao,

H. Nakai, M. Klene, X. Li, J. E. Knox, H. P. Hratchian, J. B. Cross,

C. Adamo, J. Jaramillo, R. Gomperts, R. E. Stratmann, O. Yazyev,

A. J. Austin, R. Cammi, C. Pomelli, J. W. Ochterski, P. Y. Ayala,

K. Morokuma, G. A. Voth, P. Salvador, J. J. Dannenberg,

V. G. Zakrzewski, S. Dapprich, A. D. Daniels, M. C. Strain,

O. Farkas, D. K. Malick, A. D. Rabuck, K. Raghavachari,

J. B. Foresman, J. V. Ortiz, Q. Cui, A. G. Baboul, S. Clifford,

J. Cioslowski, B. B. Stefanov, G. Liu, A. Liashenko, P. Piskorz,

I. Komaromi, R. L. Martin, D. J. Fox, T. Keith, M. A. Al-Laham,

C. Y. Peng, A. Nanayakkara, M. Challacombe, P. M. W. Gill,

B. Johnson, W. Chen, M. W. Wong, C. Gonzalez, and J. A. Pople,

Gaussian, Inc., Pittsburgh PA, 2003.

Contents:

I-XII Structures and Energies for the complexes and fragments. S2-S7

XII-XV

Calculated NMR properties for methane and the complexes

XVI-XXX Harmonic vibrational frequency analysis of methane and the complexes involving different isotopomers.

S12-S81

XXXI EIE Calculation method introduction on the multiple deuterium

substitutions

XXXII The distribution analysis of vibrational frequency changes in the case of

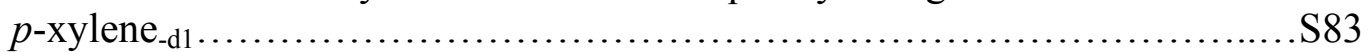

XXXIII Side-by-side table (d0/d1 models)....... 
I. MP2/6-311++G(d,p) optimized structure of methane-benzene complex:

\begin{tabular}{|c|c|c|c|c|c|}
\hline \multirow{2}{*}{$\begin{array}{l}\text { Center } \\
\text { Number }\end{array}$} & \multirow{2}{*}{$\begin{array}{l}\text { Atomic } \\
\text { Number }\end{array}$} & \multirow{2}{*}{$\begin{array}{l}\text { Atomic } \\
\text { Type }\end{array}$} & \multicolumn{3}{|c|}{ Coordinates (Angstroms) } \\
\hline & & & $\mathrm{x}$ & $\mathrm{Y}$ & Z \\
\hline 1 & 6 & 0 & .000000 & 1.400400 & -.704928 \\
\hline 2 & 1 & 0 & .000000 & 2.487200 & -.704928 \\
\hline 3 & 6 & 0 & 1.212780 & .700200 & -.704928 \\
\hline 4 & 1 & 0 & 2.153980 & 1.243600 & -.704928 \\
\hline 5 & 6 & 0 & 1.212780 & -.700200 & -.704928 \\
\hline 6 & 1 & 0 & 2.153980 & -1.243600 & -.704928 \\
\hline 7 & 6 & 0 & .000000 & -1.400400 & -.704928 \\
\hline 8 & 1 & 0 & .000000 & -2.487200 & -.704928 \\
\hline 9 & 6 & 0 & -1.212780 & -.700200 & -.704928 \\
\hline 10 & 1 & 0 & -2.153980 & -1.243600 & -.704928 \\
\hline 11 & 6 & 0 & -1.212780 & .700200 & -.704928 \\
\hline 12 & 1 & 0 & -2.153980 & 1.243600 & -.704928 \\
\hline 13 & 1 & 0 & .000000 & .000000 & 1.870572 \\
\hline 14 & 6 & 0 & .000000 & .000000 & 2.960172 \\
\hline 15 & 1 & 0 & .000000 & 1.028260 & 3.325122 \\
\hline 16 & 1 & 0 & .890500 & -.514130 & 3.325122 \\
\hline 17 & 1 & 0 & -.890500 & -.514130 & 3.325122 \\
\hline
\end{tabular}

II. MP2/6-311++G(d,p) optimized structure of methane-tetrachloromethane

\begin{tabular}{|c|c|c|c|c|c|}
\hline \multirow{2}{*}{$\begin{array}{l}\text { Center } \\
\text { Number }\end{array}$} & \multirow{2}{*}{$\begin{array}{l}\text { Atomic } \\
\text { Number }\end{array}$} & \multirow{2}{*}{$\begin{array}{l}\text { Atomic } \\
\text { Type }\end{array}$} & \multicolumn{3}{|c|}{ Coordinates (Angstroms) } \\
\hline & & & $\mathrm{X}$ & $\mathrm{Y}$ & Z \\
\hline 1 & 6 & 0 & .000000 & .000000 & -3.640747 \\
\hline 2 & 6 & 0 & .000000 & .000000 & .491653 \\
\hline 3 & 1 & 0 & 1.027696 & .000000 & -3.276312 \\
\hline 4 & 1 & 0 & -.513848 & -.890011 & -3.276312 \\
\hline 5 & 1 & 0 & -.513848 & .890011 & -3.276312 \\
\hline 6 & 17 & 0 & .846067 & 1.440255 & -.098603 \\
\hline 7 & 17 & 0 & .824264 & -1.452843 & -.098603 \\
\hline 8 & 17 & 0 & -1.670331 & .012588 & -.098603 \\
\hline 9 & 1 & 0 & .000000 & .000000 & -4.731547 \\
\hline 10 & 17 & 0 & .000000 & .000000 & 2.263753 \\
\hline
\end{tabular}


III. MP2/6-311++G(d,p) optimized structure of methane-benzene complex (reverse $\mathrm{C}_{3 \mathrm{v}}$ geometry, where three hydrogens of methane point to benzene) :

\begin{tabular}{|c|c|c|c|c|c|}
\hline \multirow{2}{*}{$\begin{array}{l}\text { Center } \\
\text { Number }\end{array}$} & \multirow{2}{*}{$\begin{array}{l}\text { Atomic } \\
\text { Number }\end{array}$} & \multirow{2}{*}{$\begin{array}{c}\text { Atomic } \\
\text { Type }\end{array}$} & \multicolumn{3}{|c|}{ Coordinates (Angstroms) } \\
\hline & & & $\mathrm{X}$ & Y & Z \\
\hline 1 & 6 & 0 & 0.000000 & 1.400236 & -0.688568 \\
\hline 2 & 1 & 0 & 0.000000 & 2.487160 & -0.688568 \\
\hline 3 & 6 & 0 & 1.212640 & 0.700118 & -0.688568 \\
\hline 4 & 1 & 0 & 2.153944 & 1.243580 & -0.688568 \\
\hline 5 & 6 & 0 & 1.212640 & -0.700118 & -0.688568 \\
\hline 6 & 1 & 0 & 2.153944 & -1.243580 & -0.688568 \\
\hline 7 & 6 & 0 & 0.000000 & -1.400236 & -0.688568 \\
\hline 8 & 1 & 0 & 0.000000 & -2.487160 & -0.688568 \\
\hline 9 & 6 & 0 & -1.212640 & -0.700118 & -0.688568 \\
\hline 10 & 1 & 0 & -2.153944 & -1.243580 & -0.688568 \\
\hline 11 & 6 & 0 & -1.212640 & 0.700118 & -0.688568 \\
\hline 12 & 1 & 0 & -2.153944 & 1.243580 & -0.688568 \\
\hline 13 & 1 & 0 & 0.000000 & 0.000000 & 3.984760 \\
\hline 14 & 6 & 0 & 0.000000 & 0.000000 & 2.893545 \\
\hline 15 & 1 & 0 & 0.000000 & 1.026128 & 2.524615 \\
\hline 16 & 1 & 0 & 0.888653 & -0.513064 & 2.524615 \\
\hline 17 & 1 & 0 & -0.888653 & -0.513064 & 2.524615 \\
\hline
\end{tabular}

EUMP2 $=\quad-0.27196733448569 \mathrm{D}+03$

IV. MP2/6-311++G(d,p) optimized structure of methane-tetrachloromethane (reverse $\mathrm{C}_{3 \mathrm{v}}$ geometry, where three hydrogens of methane point to benzene) :

\begin{tabular}{|c|c|c|c|c|c|}
\hline \multirow{2}{*}{$\begin{array}{l}\text { Center } \\
\text { Number }\end{array}$} & \multirow{2}{*}{$\begin{array}{l}\text { Atomic } \\
\text { Number }\end{array}$} & \multirow{2}{*}{$\begin{array}{l}\text { Atomic } \\
\text { Type }\end{array}$} & \multicolumn{3}{|c|}{ Coordinates (Angstroms) } \\
\hline & & & $\mathrm{X}$ & Y & Z \\
\hline 1 & 6 & 0 & 0.000000 & 0.000000 & -3.950889 \\
\hline 2 & 6 & 0 & 0.000000 & 0.000000 & 0.534236 \\
\hline 3 & 1 & 0 & 0.889959 & 0.515425 & -4.313557 \\
\hline 4 & 1 & 0 & 0.001392 & -1.028440 & -4.313557 \\
\hline 5 & 1 & 0 & -0.891351 & 0.513015 & -4.313557 \\
\hline 6 & 17 & 0 & 0.000000 & 1.670107 & -0.056982 \\
\hline 7 & 17 & 0 & 1.446355 & -0.835053 & -0.056982 \\
\hline 8 & 17 & 0 & -1.446355 & -0.835053 & -0.056982 \\
\hline 9 & 1 & 0 & 0.000000 & 0.000000 & -2.861649 \\
\hline 10 & 17 & 0 & 0.000000 & 0.000000 & 2.306372 \\
\hline
\end{tabular}

EUMP2 $=-0.19169524816025 \mathrm{D}+04$ 
V. $\quad$ MP2/6-311++G(d,p) single point energy of the methane in the methanebenzene complex

\begin{tabular}{|c|c|c|c|c|c|}
\hline Center & Atomic & Atomic & \multicolumn{3}{|c|}{ Coordinates (Angstroms) } \\
\hline Number & Number & Type & $\mathrm{X}$ & Y & Z \\
\hline 1 & 1 & 0 & 0.000000 & 0.000000 & 1.870572 \\
\hline 2 & 6 & 0 & 0.000000 & 0.000000 & 2.960172 \\
\hline 3 & 1 & 0 & 0.000000 & 1.028260 & 3.325122 \\
\hline 4 & 1 & 0 & 0.890500 & -0.514130 & 3.325122 \\
\hline 5 & 1 & 0 & -0.890500 & -0.514130 & 3.325122 \\
\hline
\end{tabular}

EUMP2 $=-0.40379635946648 \mathrm{D}+02$

EUMP2 = $-0.40380313268078 \mathrm{D}+02$ (counterpoise method)

VI. MP2/6-311++G(d,p) single point energy of the benzene in the methanebenzene complex

\begin{tabular}{|c|c|c|c|c|c|}
\hline \multirow{2}{*}{$\begin{array}{l}\text { Center } \\
\text { Number }\end{array}$} & \multirow{2}{*}{$\begin{array}{l}\text { Atomic } \\
\text { Number }\end{array}$} & \multirow{2}{*}{$\begin{array}{l}\text { Atomic } \\
\text { Type }\end{array}$} & \multicolumn{3}{|c|}{ Coordinates (Angstroms) } \\
\hline & & & $\mathrm{X}$ & $\mathrm{Y}$ & Z \\
\hline 1 & 6 & 0 & 0.000000 & 1.400400 & -0.704928 \\
\hline 2 & 1 & 0 & 0.000000 & 2.487200 & -0.704928 \\
\hline 3 & 6 & 0 & 1.212780 & 0.700200 & -0.704928 \\
\hline 4 & 1 & 0 & 2.153980 & 1.243600 & -0.704928 \\
\hline 5 & 6 & 0 & 1.212780 & -0.700200 & -0.704928 \\
\hline 6 & 1 & 0 & 2.153980 & -1.243600 & -0.704928 \\
\hline 7 & 6 & 0 & 0.000000 & -1.400400 & -0.704928 \\
\hline 8 & 1 & 0 & 0.000000 & -2.487200 & -0.704928 \\
\hline 9 & 6 & 0 & -1.212780 & -0.700200 & -0.704928 \\
\hline 10 & 1 & 0 & -2.153980 & -1.243600 & -0.704928 \\
\hline 11 & 6 & 0 & -1.212780 & 0.700200 & -0.704928 \\
\hline 12 & 1 & 0 & -2.153980 & 1.243600 & -0.704928 \\
\hline
\end{tabular}

EUMP2 $=-0.23158425732303 \mathrm{D}+03$

EUMP2 $=-0.23158645958649 \mathrm{D}+03$ (counterpoise method) 
VII. MP2/6-311++G(d,p) single point energy of the methane in the methanetetrachloromethane complex

\begin{tabular}{|c|c|c|c|c|c|}
\hline \multirow{2}{*}{$\begin{array}{l}\text { Center } \\
\text { Number }\end{array}$} & \multirow{2}{*}{$\begin{array}{l}\text { Atomic } \\
\text { Number }\end{array}$} & \multirow{2}{*}{$\begin{array}{c}\text { Atomic } \\
\text { Type }\end{array}$} & \multicolumn{3}{|c|}{ Coordinates (Angstroms) } \\
\hline & & & $\mathrm{X}$ & Y & Z \\
\hline 1 & 6 & 0 & 0.000000 & 0.000000 & -3.640747 \\
\hline 2 & 1 & 0 & 1.027696 & 0.000000 & -3.276312 \\
\hline 3 & 1 & 0 & -0.513848 & -0.890011 & -3.276312 \\
\hline 4 & 1 & 0 & -0.513848 & 0.890011 & -3.276312 \\
\hline 5 & 1 & 0 & 0.000000 & 0.000000 & -4.731547 \\
\hline UMP2 = & & $\mathrm{D}+02$ & & & \\
\hline EUMP2 = & -0.40 & $46888 D+02$ & unterpoise & thod) & \\
\hline
\end{tabular}

VIII. MP2/6-311++G(d,p) single point energy of the tetrachloromethane in the methane-tetrachloromethane complex.

\begin{tabular}{|c|c|c|c|c|c|}
\hline \multirow{2}{*}{$\begin{array}{l}\text { Center } \\
\text { Number }\end{array}$} & \multirow{2}{*}{$\begin{array}{l}\text { Atomic } \\
\text { Number }\end{array}$} & \multirow{2}{*}{$\begin{array}{c}\text { Atomic } \\
\text { Type }\end{array}$} & \multicolumn{3}{|c|}{ Coordinates (Angstroms) } \\
\hline & & & $\mathrm{X}$ & $\mathrm{Y}$ & Z \\
\hline \multicolumn{6}{|c|}{-----------------------------------------------------------------------} \\
\hline 1 & 6 & 0 & 0.000000 & 0.000000 & 0.491653 \\
\hline 2 & 17 & 0 & 0.846067 & 1.440255 & -0.098603 \\
\hline 3 & 17 & 0 & 0.824264 & -1.452843 & -0.098603 \\
\hline 4 & 17 & 0 & -1.670331 & 0.012588 & -0.098603 \\
\hline 5 & 17 & 0 & 0.000000 & 0.000000 & 2.263753 \\
\hline \multicolumn{6}{|c|}{ 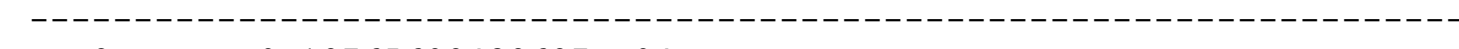 } \\
\hline $\mathrm{P} 2=$ & & $87 D+04$ & & & \\
\hline IP2 $=$ & -0.187 & $339557 \mathrm{D}+04$ & anterpoise & thod) & \\
\hline
\end{tabular}


IX. MP2/6-311++G(d,p) single point energy of the methane in the methanebenzene complex (reverse $\mathrm{C}_{3 \mathrm{v}}$ geometry)

\begin{tabular}{|c|c|c|c|c|c|}
\hline \multirow{2}{*}{$\begin{array}{l}\text { Center } \\
\text { Number }\end{array}$} & \multirow{2}{*}{$\begin{array}{l}\text { Atomic } \\
\text { Number }\end{array}$} & \multirow{2}{*}{$\begin{array}{c}\text { Atomic } \\
\text { Type }\end{array}$} & \multicolumn{3}{|c|}{ Coordinates (Angstroms) } \\
\hline & & & $\mathrm{X}$ & $\mathrm{Y}$ & Z \\
\hline 1 & 1 & 0 & 0.000000 & 0.000000 & 3.979880 \\
\hline 2 & 6 & 0 & 0.000000 & 0.000000 & 2.888761 \\
\hline 3 & 1 & 0 & 0.888572 & 0.513018 & 2.519494 \\
\hline 4 & 1 & 0 & 0.000000 & -1.026035 & 2.519494 \\
\hline 5 & 1 & 0 & -0.888572 & 0.513018 & 2.519494 \\
\hline $2=$ & -0 . & $1511 D$ & & & \\
\hline EUMP2 = & -0.403 & $8078 \mathrm{D}+$ & terpoise & (od) & \\
\hline
\end{tabular}

\begin{tabular}{|c|c|c|c|c|c|}
\hline \multirow{2}{*}{$\begin{array}{l}\text { Center } \\
\text { Number }\end{array}$} & \multirow{2}{*}{$\begin{array}{l}\text { Atomic } \\
\text { Number }\end{array}$} & \multirow{2}{*}{$\begin{array}{c}\text { Atomic } \\
\text { Type }\end{array}$} & \multicolumn{3}{|c|}{ Coordinates (Angstroms) } \\
\hline & & & $\mathrm{X}$ & $\mathrm{Y}$ & Z \\
\hline & & & ------ & -------- & ----------- \\
\hline 1 & 6 & 0 & 0.700116 & 1.212637 & -0.687403 \\
\hline 2 & 1 & 0 & 1.243579 & 2.153942 & -0.687403 \\
\hline 3 & 6 & 0 & 1.400232 & 0.000000 & -0.687403 \\
\hline 4 & 1 & 0 & 2.487158 & 0.000000 & -0.687403 \\
\hline 5 & 6 & 0 & 0.700116 & -1.212637 & -0.687403 \\
\hline 6 & 1 & 0 & 1.243579 & -2.153942 & -0.687403 \\
\hline 7 & 6 & 0 & -0.700116 & -1.212637 & -0.687403 \\
\hline 8 & 1 & 0 & -1.243579 & -2.153942 & -0.687403 \\
\hline 9 & 6 & 0 & -1.400232 & 0.000000 & -0.687403 \\
\hline 10 & 1 & 0 & -2.487158 & 0.000000 & -0.687403 \\
\hline 11 & 6 & 0 & -0.700116 & 1.212637 & -0.687403 \\
\hline 12 & 1 & 0 & -1.243579 & 2.153942 & -0.687403 \\
\hline
\end{tabular}

EUMP2 $=-0.23158425738827 \mathrm{D}+03$

EUMP2 = $\quad-0.23158645958649 \mathrm{D}+03$ (counterpoise method) 
XI. MP2/6-311++G(d,p) single point energy of the methane in the methanetetrachloromethane complex (reverse $\mathrm{C}_{3 \mathrm{v}}$ geometry)

\begin{tabular}{|c|c|c|c|c|c|}
\hline \multirow{2}{*}{$\begin{array}{l}\text { Center } \\
\text { Number }\end{array}$} & \multirow{2}{*}{$\begin{array}{l}\text { Atomic } \\
\text { Number }\end{array}$} & \multirow{2}{*}{$\begin{array}{c}\text { Atomic } \\
\text { Type }\end{array}$} & \multicolumn{3}{|c|}{ Coordinates (Angstroms) } \\
\hline & & & $\mathrm{X}$ & Y & Z \\
\hline-- & & & -- & ----- & ---------- \\
\hline 1 & 6 & 0 & 0.000000 & 0.000000 & -3.950889 \\
\hline 2 & 1 & 0 & 0.889959 & 0.515425 & -4.313557 \\
\hline 3 & 1 & 0 & 0.001392 & -1.028440 & -4.313557 \\
\hline 4 & 1 & 0 & -0.891351 & 0.513015 & -4.313557 \\
\hline 5 & 1 & 0 & 0.000000 & 0.000000 & -2.861649 \\
\hline
\end{tabular}

EUMP2 $=-0.40379636842891 \mathrm{D}+02$

EUMP2 $=\quad-0.40379969797603 \mathrm{D}+02$ (counterpoise method)

XII. MP2/6-311++G(d,p) single point energy of the tetrachloromethane in the methane-tetrachloromethane complex (reverse $\mathrm{C}_{3 \mathrm{v}}$ geometry)

\begin{tabular}{|c|c|c|c|c|c|}
\hline \multirow{2}{*}{$\begin{array}{l}\text { Center } \\
\text { Number }\end{array}$} & \multirow{2}{*}{$\begin{array}{l}\text { Atomic } \\
\text { Number }\end{array}$} & \multirow{2}{*}{$\begin{array}{c}\text { Atomic } \\
\text { Type }\end{array}$} & \multicolumn{3}{|c|}{ Coordinates (Angstroms) } \\
\hline & & & $\mathrm{X}$ & $\mathrm{Y}$ & Z \\
\hline------ & & & $2-5-2=$ & ------- & ---------- \\
\hline 1 & 6 & 0 & 0.000000 & 0.000000 & 0.534236 \\
\hline 2 & 17 & 0 & 0.000000 & 1.670107 & -0.056982 \\
\hline 3 & 17 & 0 & 1.446355 & -0.835053 & -0.056982 \\
\hline 4 & 17 & 0 & -1.446355 & -0.835053 & -0.056982 \\
\hline 5 & 17 & 0 & 0.000000 & 0.000000 & 2.306372 \\
\hline
\end{tabular}

EUMP2 $=\quad-0.18765698436483 \mathrm{D}+04$

EUMP2 = $-0.18765722269617 D+04$ (counterpoise method) 
XIII. MP2/6-311++G(d,p) optimized structure of methane and it calculated NMR properties ( $\mathrm{H}$ Isotropic $=31.6705$ )

\begin{tabular}{|c|c|c|c|c|c|}
\hline \multirow{2}{*}{$\begin{array}{l}\text { Center } \\
\text { Number }\end{array}$} & \multirow{2}{*}{$\begin{array}{l}\text { Atomic } \\
\text { Number }\end{array}$} & \multirow{2}{*}{$\begin{array}{c}\text { Atomic } \\
\text { Type }\end{array}$} & \multicolumn{3}{|c|}{ Coordinates (Angstroms) } \\
\hline & & & $\mathrm{X}$ & Y & Z \\
\hline & & & & - & --------- \\
\hline 1 & 1 & 0 & 0.000000 & 0.000000 & 0.000000 \\
\hline 2 & 6 & 0 & 0.000000 & 0.000000 & 1.089000 \\
\hline 3 & 1 & 0 & 1.026719 & 0.000000 & 1.452000 \\
\hline 4 & 1 & 0 & -0.513360 & 0.889165 & 1.452000 \\
\hline 5 & 1 & 0 & -0.513360 & -0.889165 & 1.452000 \\
\hline
\end{tabular}

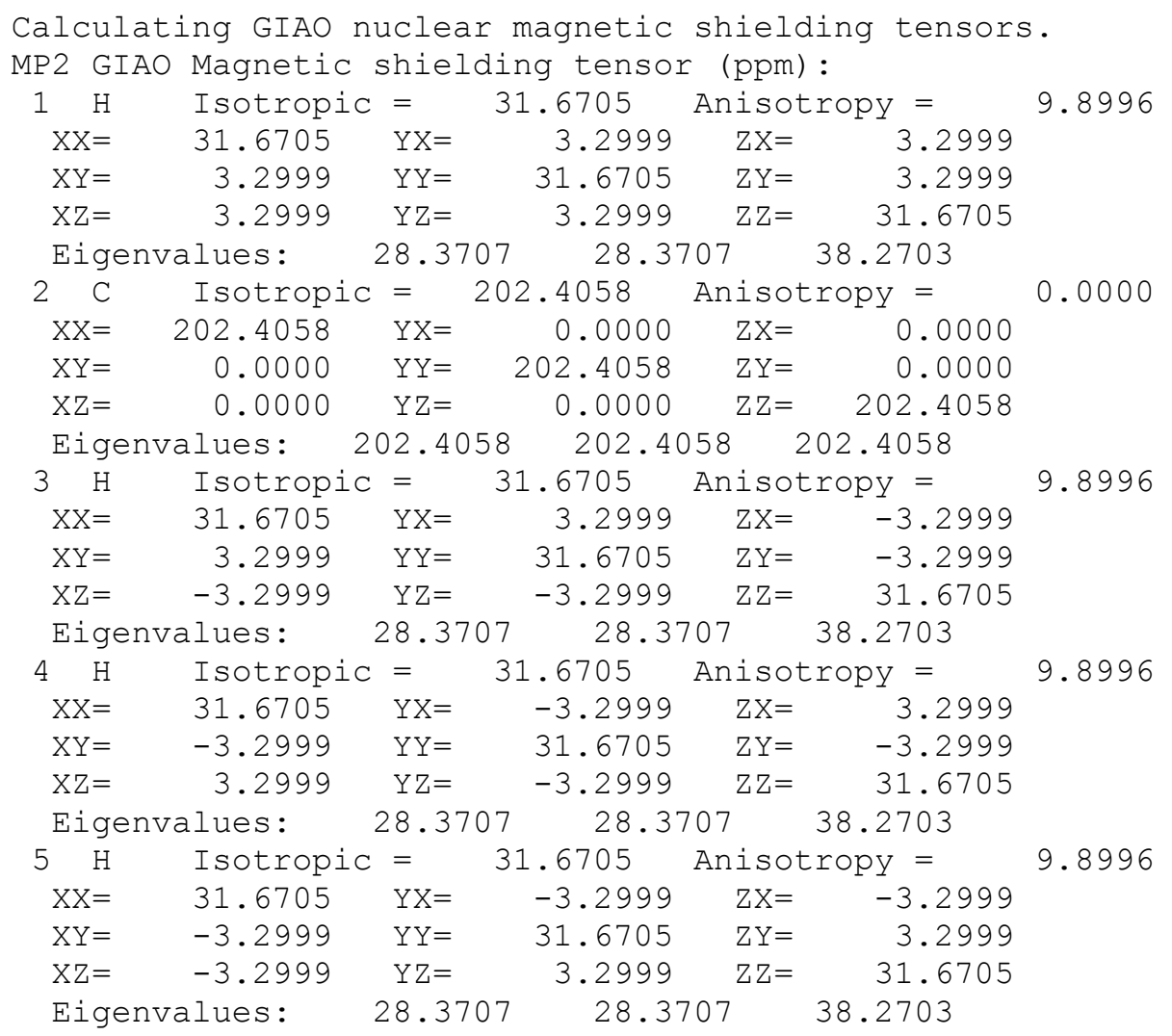


XIV. The calculated NMR properties for the MP2/6-311++G(d,P) optimized methane-benzene complex (H13 is the hydrogen facing the aromatic ring, Isotropic $=34.4928$ )

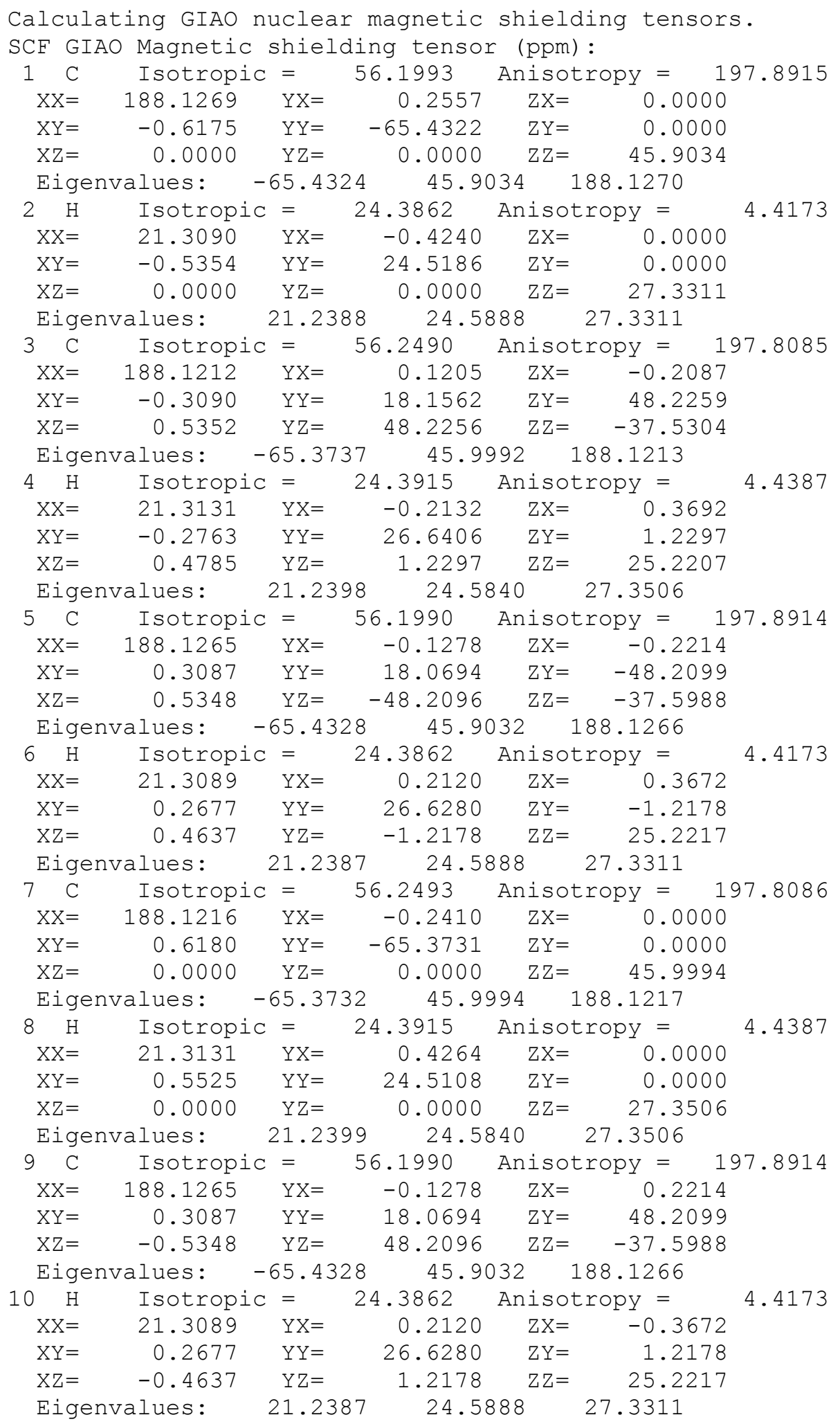




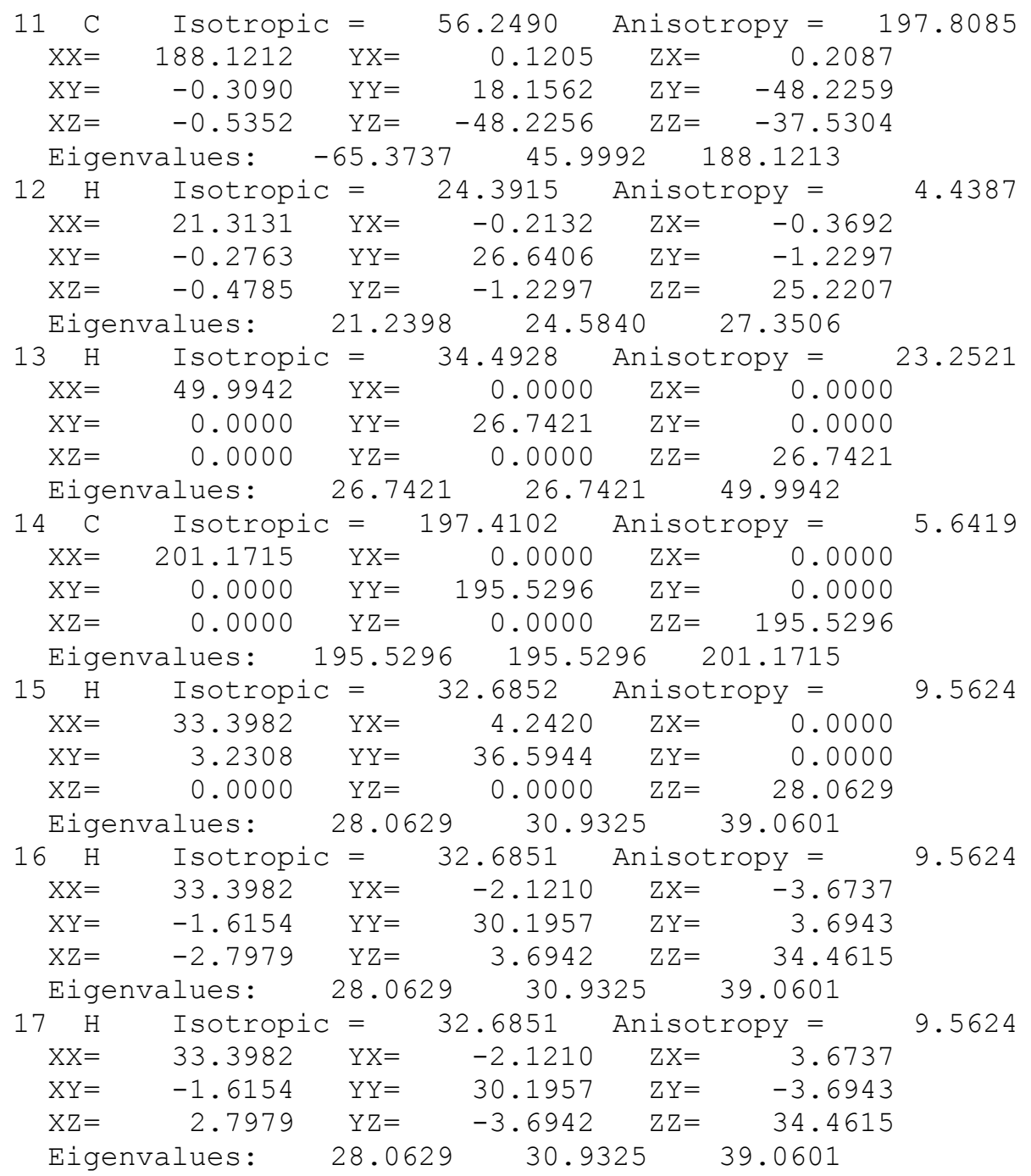


XV. The calculated NMR properties for the MP2/6-311++G(d,P) optimized methane-tetrachloromethane complex (H3, H4, and H5 are the hydrogens facing tetrachloromethane, Isotropic $=31.4511$ )

Calculating GIAO nuclear magnetic shielding tensors. SCF GIAO Magnetic shielding tensor (ppm):

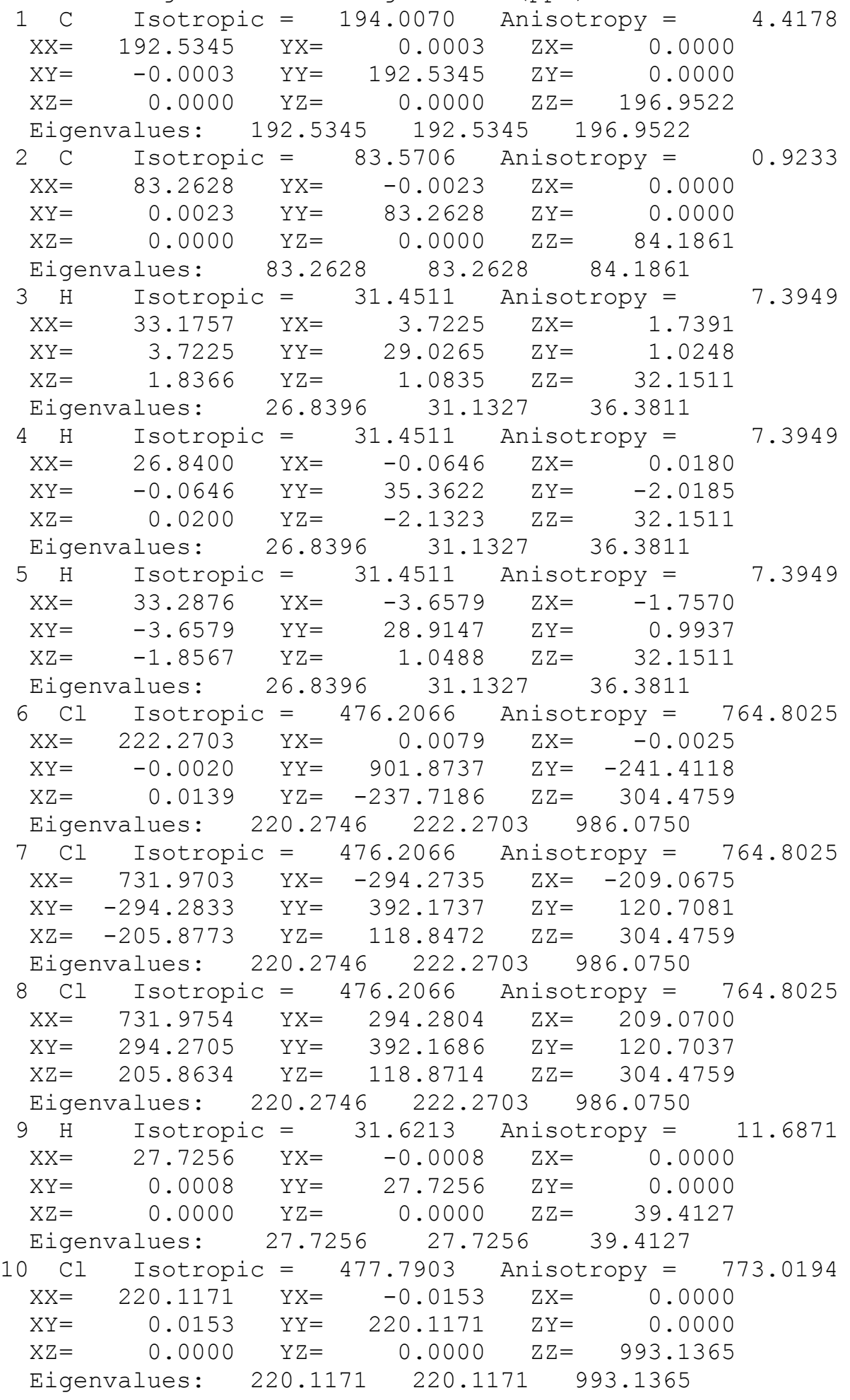


XVI. Harmonic frequency analysis and zero-point energy for non-deuterated methane with the MP2/6-311++G $(d, p)$ method:

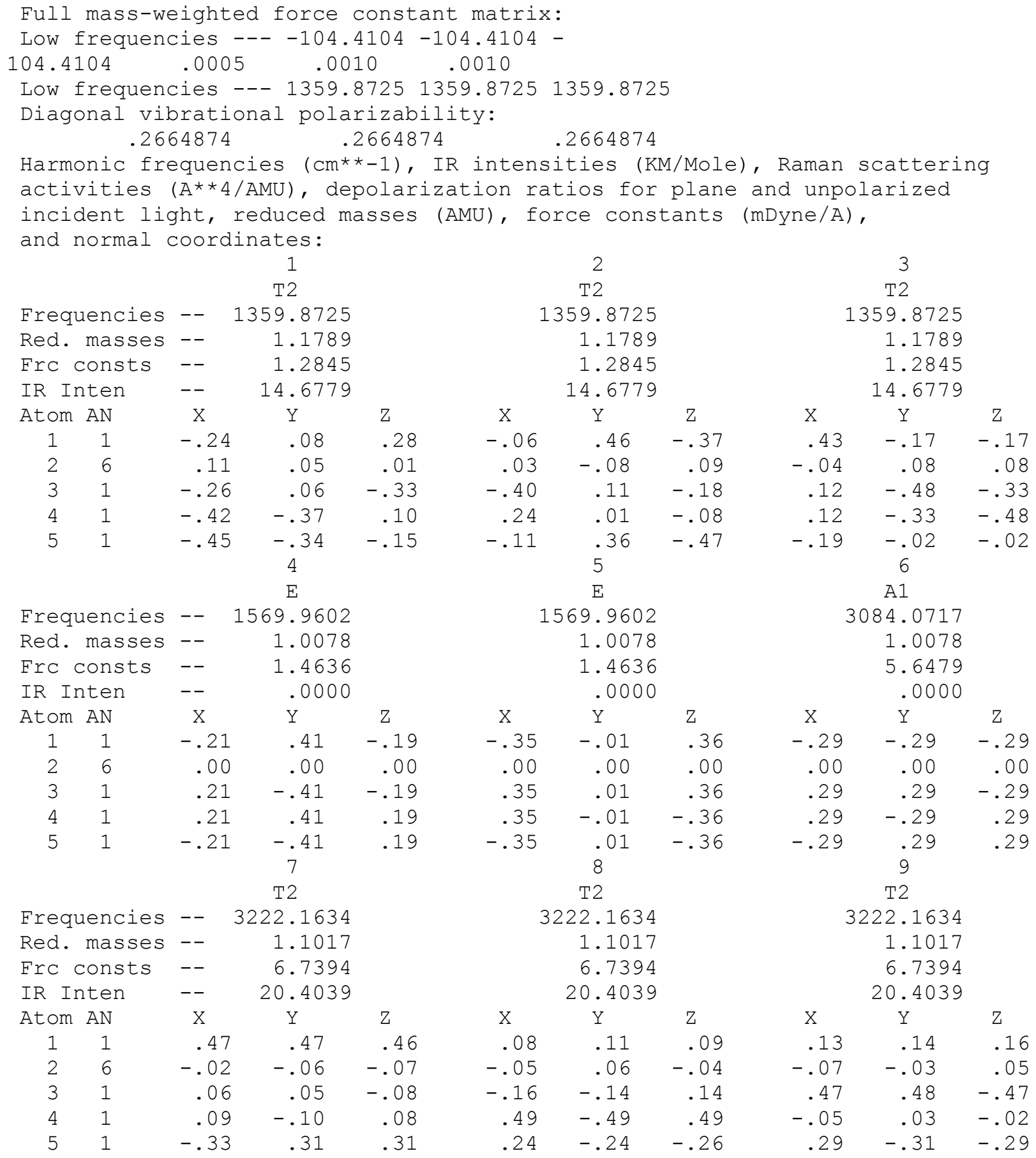

\section{- Thermochemistry -}

Temperature 298.150 Kelvin. Pressure 1.00000 Atm. Atom 1 has atomic number 1 and mass 1.00783 Atom 2 has atomic number 6 and mass 12.00000 


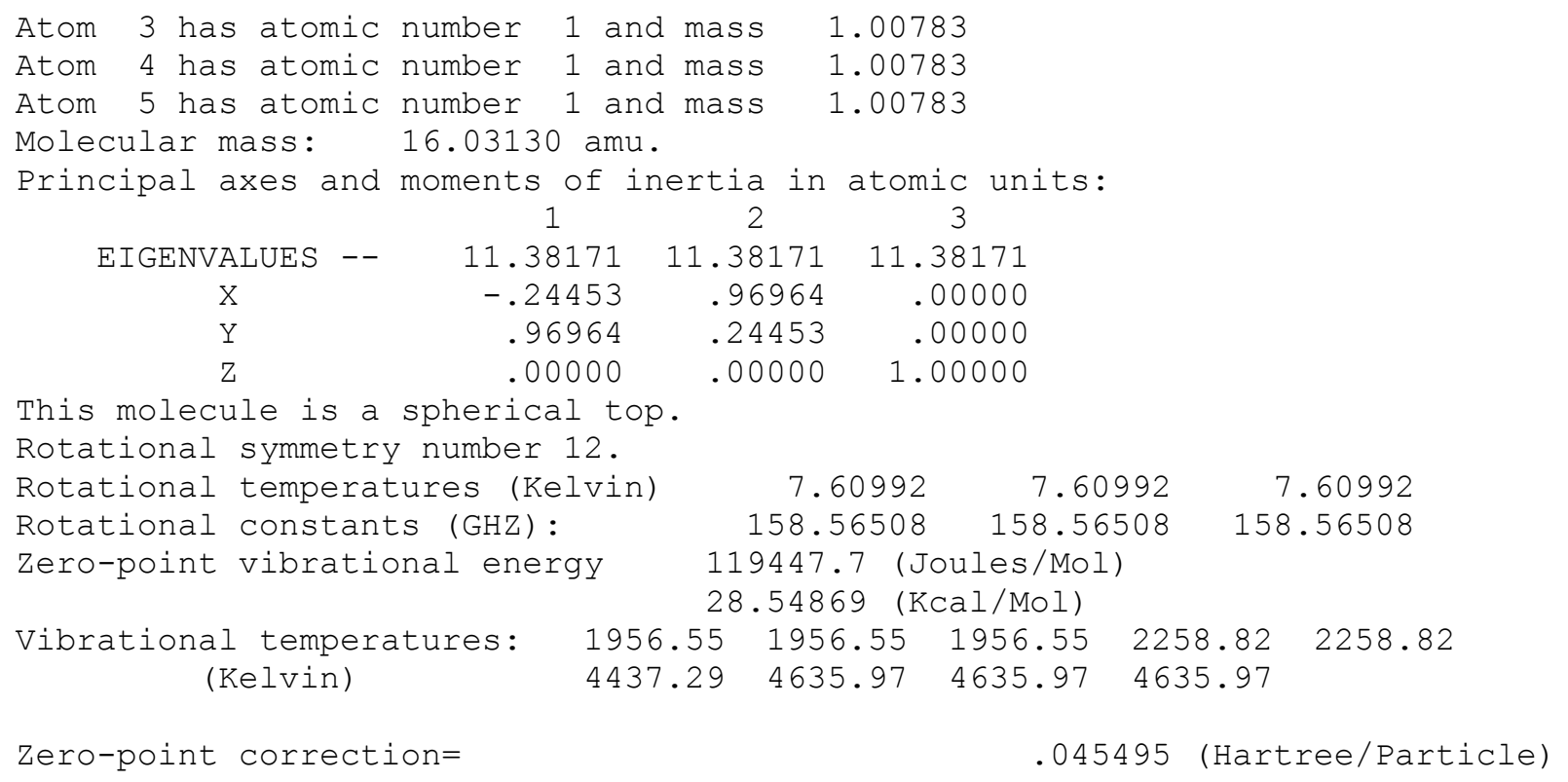


XVII. Harmonic frequency analysis and zero-point energy for monodeuterated methane with the MP2/6-311++G $(d, p)$ method:

\begin{tabular}{|c|c|c|c|c|c|c|c|c|c|}
\hline \multicolumn{10}{|c|}{ Low frequencies --- -104.4104 -89.8092 } \\
\hline \multicolumn{10}{|l|}{89.8092} \\
\hline \multicolumn{10}{|c|}{ Low frequencies --- 1198.75391198 .75391353 .9509} \\
\hline \multicolumn{10}{|c|}{ Diagonal vibrational polarizability: } \\
\hline \multirow{2}{*}{\multicolumn{10}{|c|}{$\begin{array}{l}\left.\text { Harmonic frequencies ( } \mathrm{cm}^{*}-1\right) \text {, IR intensities (KM/Mole), Raman scattering } \\
\text { activities (A**4/AMU), depolarization ratios for plane and unpolarized } \\
\text { incident light, reduced masses (AMU), force constants (mDyne/A), } \\
\text { and normal coordinates: }\end{array}$}} \\
\hline & & & & & & & & & \\
\hline \multicolumn{4}{|c|}{1} & \multicolumn{3}{|c|}{2} & \multicolumn{3}{|c|}{3} \\
\hline \multicolumn{4}{|c|}{ ?A } & \multicolumn{3}{|c|}{ ?A } & \multicolumn{3}{|c|}{ ?A } \\
\hline Frequencies & -- & 1198.752 & & \multicolumn{3}{|c|}{1198.7523} & \multicolumn{3}{|c|}{1353.9509} \\
\hline Red. masses & -- & 1.508 & & \multicolumn{3}{|c|}{1.5087} & \multicolumn{3}{|c|}{1.1645} \\
\hline \multirow{2}{*}{$\begin{array}{l}\text { Frc consts } \\
\text { IR Inten }\end{array}$} & -- & 1.277 & & \multicolumn{3}{|c|}{1.2773} & \multicolumn{3}{|c|}{1.2578} \\
\hline & -- & 9.770 & & \multicolumn{3}{|c|}{9.7705} & \multicolumn{3}{|c|}{15.1311} \\
\hline Atom AN & $\mathrm{X}$ & $\mathrm{Y}$ & Z & $\mathrm{x}$ & $\mathrm{Y}$ & Z & $\mathrm{X}$ & $\mathrm{Y}$ & Z \\
\hline 1 & -.01 & -.35 & .37 & .42 & -.22 & -.20 & .06 & .06 & .06 \\
\hline 6 & .00 & .10 & -.11 & -.12 & .06 & .06 & .07 & .07 & .07 \\
\hline 1 & .39 & .12 & .34 & .12 & -.39 & -.18 & -.22 & -.22 & -.48 \\
\hline 1 & -.40 & -.32 & -.09 & .09 & -.20 & -.40 & -.22 & -.48 & -.22 \\
\hline \multirow[t]{3}{*}{1} & -.01 & -.30 & .28 & .38 & .28 & .30 & -.48 & -.22 & -.22 \\
\hline & & 4 & & & 5 & & & 6 & \\
\hline & & ?A & & & ?A & & & ?A & \\
\hline Frequencies & -- & 1510.017 & & & 10.017 & & & 329.2764 & \\
\hline Red. masses & -- & 1.047 & & & 1.047 & & & 2.2143 & \\
\hline Frc consts & -- & 1.407 & & & 1.407 & & & 7.0783 & \\
\hline IR Inten & -- & 2.643 & & & 2.643 & & & 5.4589 & \\
\hline Atom AN & $\mathrm{x}$ & $\mathrm{Y}$ & $\mathrm{Z}$ & $\mathrm{x}$ & $\mathrm{Y}$ & Z & $\mathrm{x}$ & $\mathrm{Y}$ & $\mathrm{Z}$ \\
\hline $1 \quad 1$ & .12 & -.08 & -.04 & .02 & .09 & -.11 & .56 & .56 & .56 \\
\hline 6 & .03 & -.02 & -.01 & .01 & .03 & -.03 & -.09 & -.09 & -.09 \\
\hline 1 & -.51 & .40 & -.12 & .02 & -.31 & -.34 & -.06 & -.06 & .06 \\
\hline 1 & -.47 & -.23 & .27 & -.19 & .28 & .43 & -.06 & .06 & -.06 \\
\hline 1 & .36 & .24 & .07 & .06 & -.45 & .51 & .06 & -.06 & -.06 \\
\hline & & 7 & & & 8 & & & 9 & \\
\hline & & ?A & & & ?A & & & ?A & \\
\hline Frequencies & -- & 3124.767 & & & 21.934 & & & 221.9341 & \\
\hline Red. masses & -- & 1.041 & & & 1.102 & & & 1.1021 & \\
\hline Frc consts & -- & 5.989 & & & 6.740 & & & 6.7406 & \\
\hline IR Inten & -- & 6.271 & & & $20.20 s$ & & & 20.2097 & \\
\hline Atom AN & $\mathrm{X}$ & $\mathrm{Y}$ & Z & $\mathrm{x}$ & $\mathrm{Y}$ & Z & $\mathrm{x}$ & $\mathrm{Y}$ & $\mathrm{Z}$ \\
\hline $\begin{array}{lll}1 & 1\end{array}$ & .03 & .03 & .03 & -.01 & .01 & .00 & .00 & .00 & .01 \\
\hline 2 & -.03 & -.03 & -.03 & -.06 & .07 & -.01 & -.04 & -.03 & .08 \\
\hline 3 & .33 & .33 & -.34 & -.05 & -.02 & .03 & .47 & .47 & -.46 \\
\hline 1 & .33 & -.34 & .33 & .42 & -.42 & .43 & -.22 & .20 & -.19 \\
\hline 51 & -.34 & .33 & .33 & .39 & -.38 & -.40 & .26 & -.27 & -.25 \\
\hline
\end{tabular}

- Thermochemistry -

Temperature 298.150 Kelvin. Pressure 1.00000 Atm.

Atom 1 has atomic number 1 and mass 2.01410

Atom 2 has atomic number 6 and mass 12.00000 


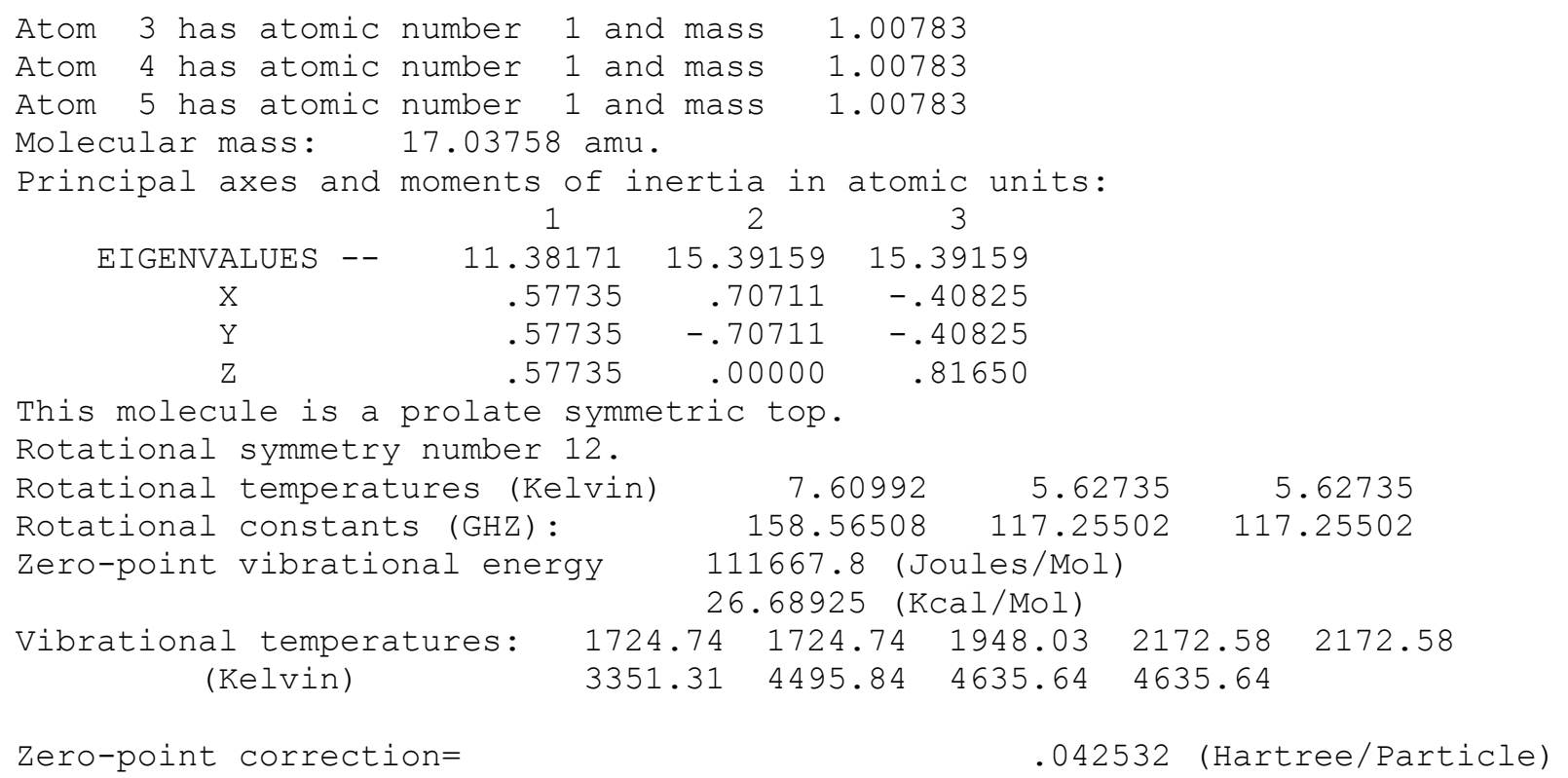


XVIII. Harmonic frequency analysis and zero-point energy for dideuterated methane with the MP2/6-311++G $(d, p)$ method:

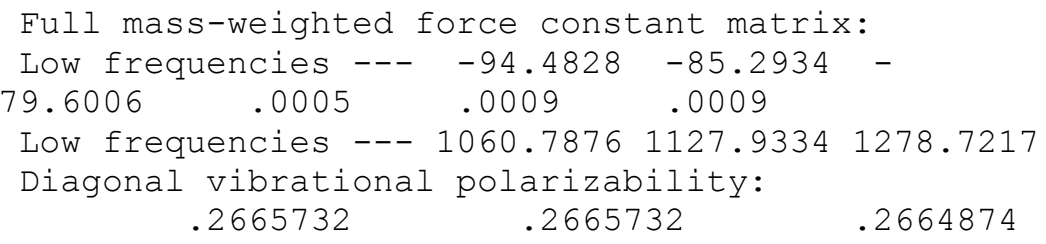

Harmonic frequencies ( $\left.\mathrm{cm}^{*} *-1\right)$, IR intensities (KM/Mole), Raman scattering activities ( $A^{* * 4 / A M U), ~ d e p o l a r i z a t i o n ~ r a t i o s ~ f o r ~ p l a n e ~ a n d ~ u n p o l a r i z e d ~}$ incident light, reduced masses (AMU), force constants (mDyne/A), and normal coordinates:

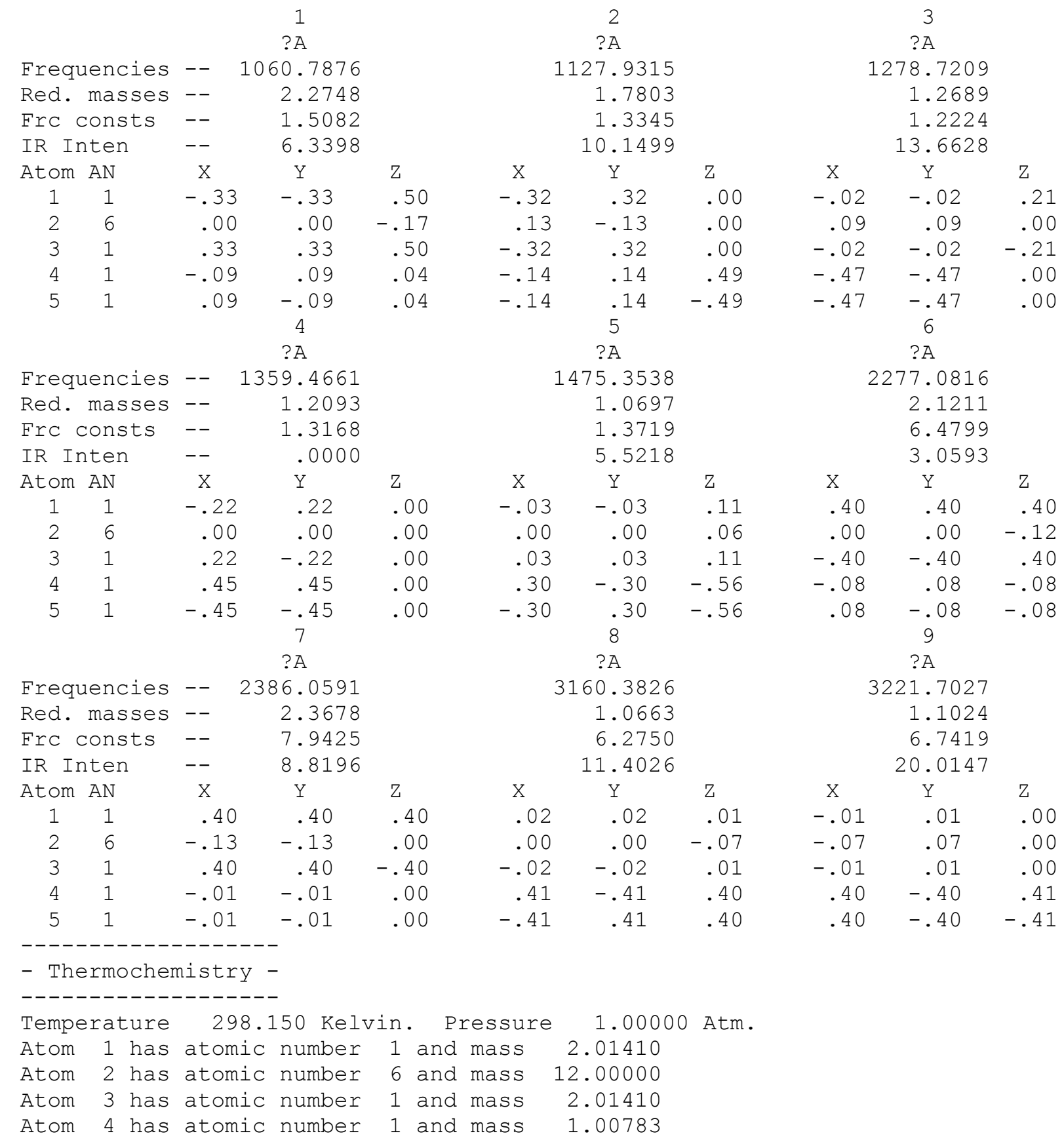




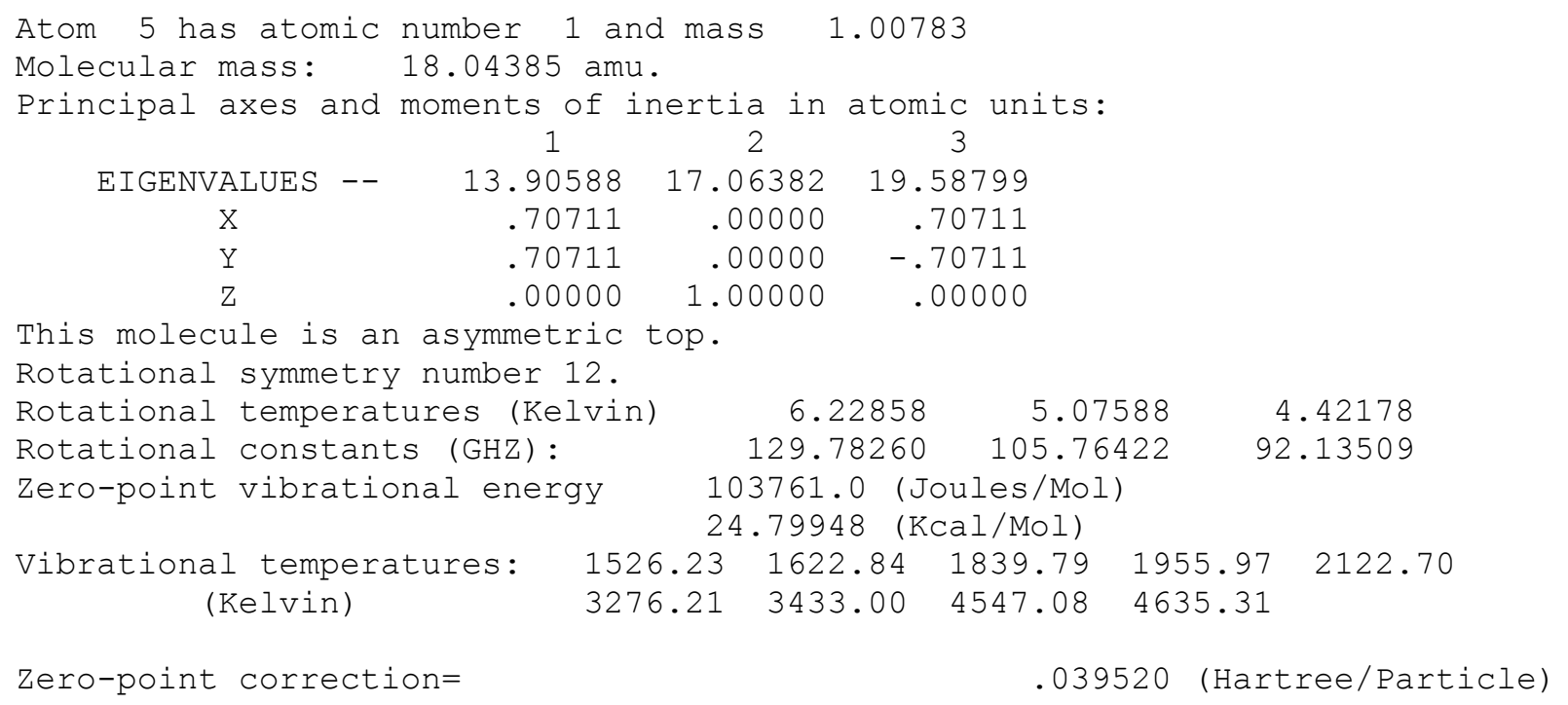


XIX. Harmonic frequency analysis and zero-point energy for tri-deuterated methane with the MP2/6-311++G $(d, p)$ method:

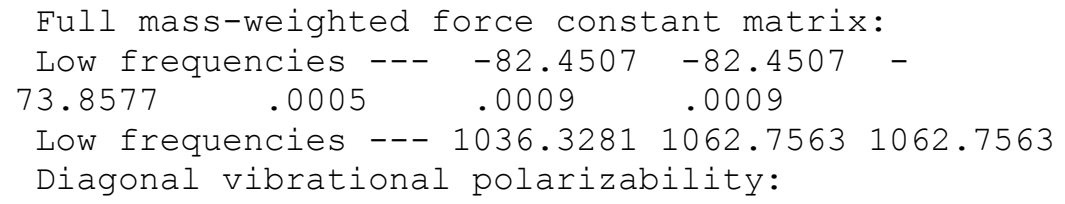




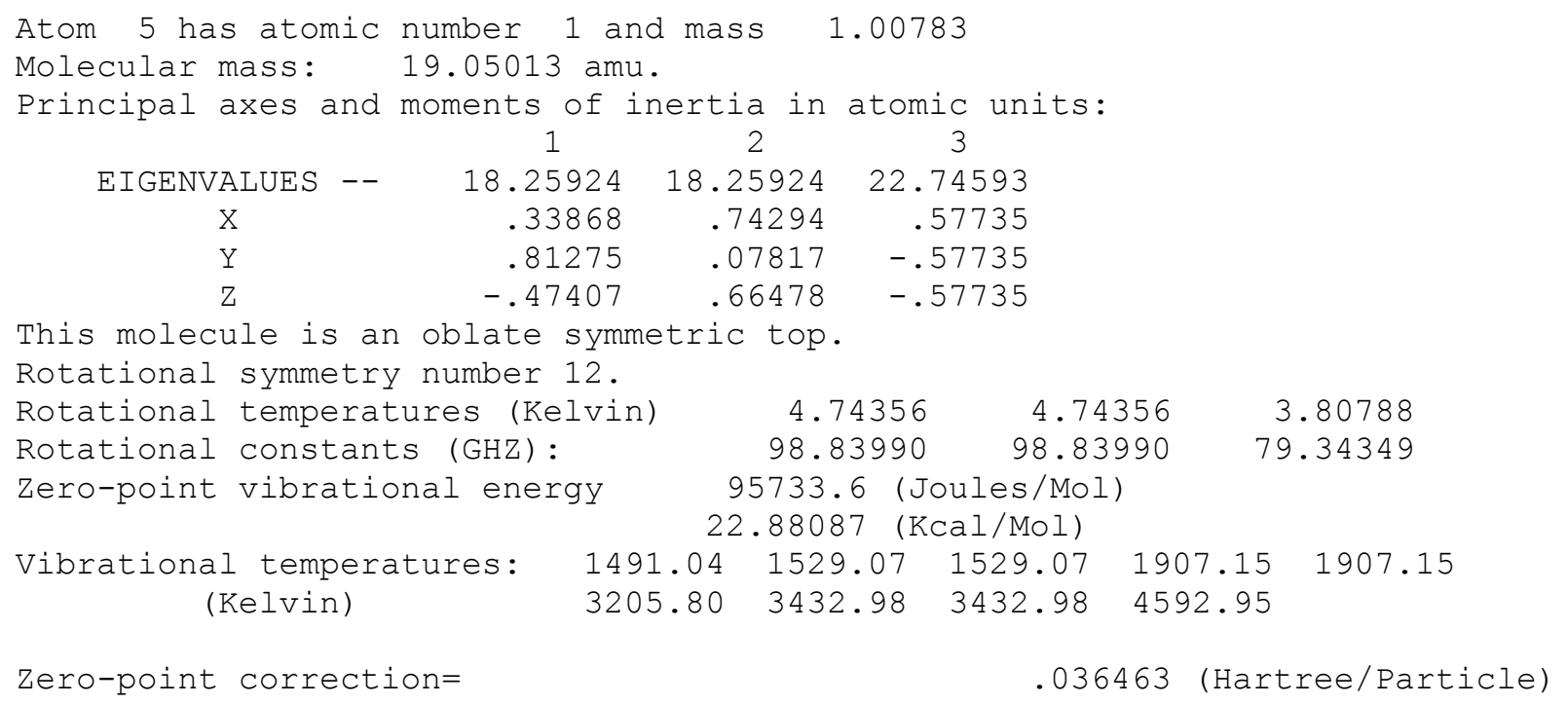


xx. Harmonic frequency analysis and zero-point energy for tetradeuterated methane with the MP2/6-311++G(d,p) method:

$\begin{array}{cccc}\text { Low frequencies }--- & -73.8577 & -73.8577 & - \\ 73.8577 \quad .0005 & .0009 & .0009 & \\ \text { Low frequencies - - } & 1027.8208 & 1027.8208 & 1027.8208 \\ \text { Diagonal vibrational polarizability: } & \\ .2664874 & .2664874 & .2664874\end{array}$

Harmonic frequencies ( $\left.\mathrm{cm}^{*} *-1\right)$, IR intensities (KM/Mole), Raman scattering activities (A**4/AMU), depolarization ratios for plane and unpolarized incident light, reduced masses (AMU), force constants (mDyne/A), and normal coordinates:

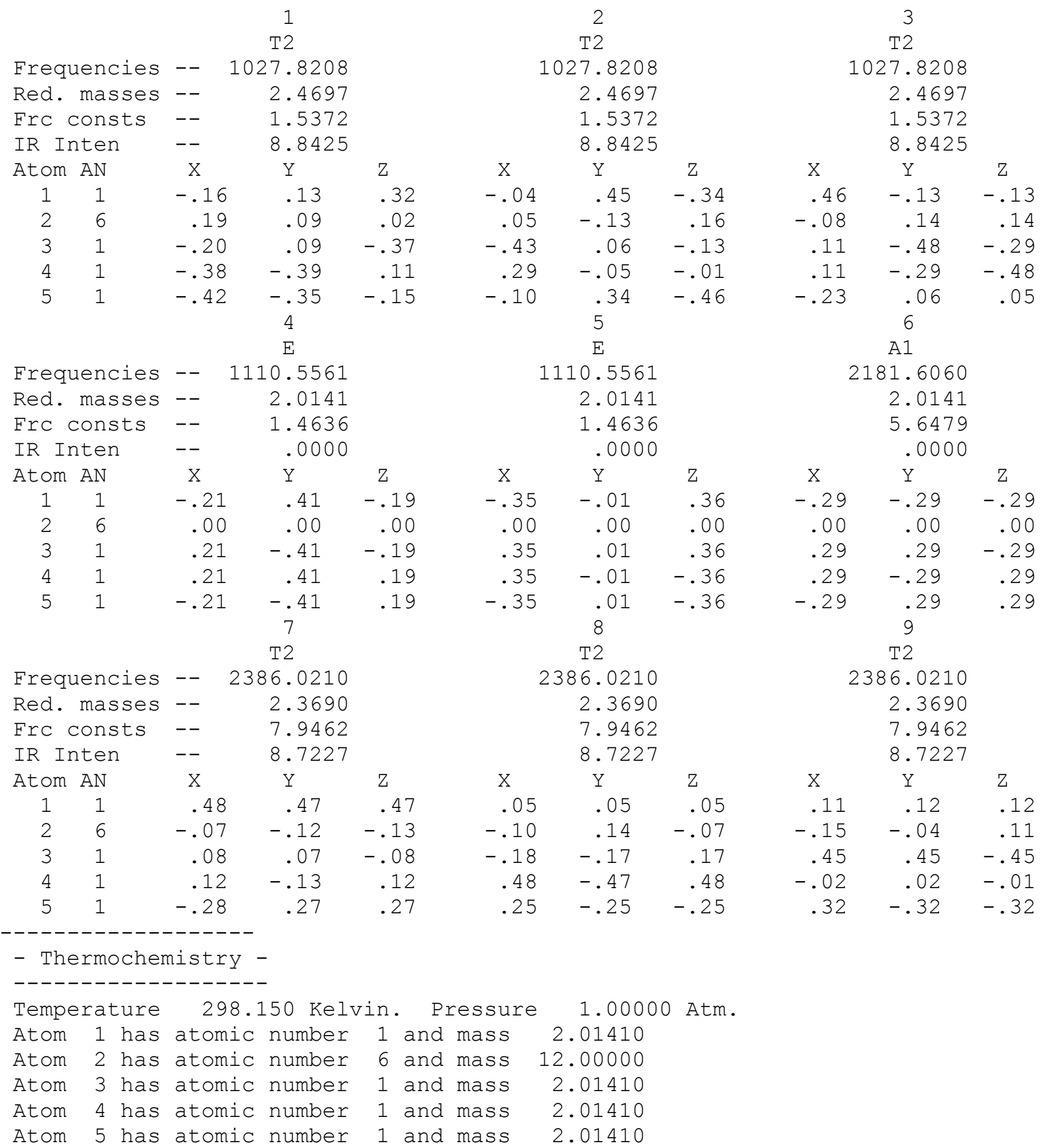


Molecular mass: 20.05641 amu.

Principal axes and moments of inertia in atomic units:

\begin{tabular}{|c|c|c|c|}
\hline & 1 & 2 & 3 \\
\hline EIGENVALUES - & 22.74593 & 22.74593 & 22.74593 \\
\hline X & .00000 & .61105 & .79159 \\
\hline Y & .00000 & .79159 & -.6110 \\
\hline Z & 1.00000 & .00000 & .0000 \\
\hline
\end{tabular}

This molecule is a spherical top.

Rotational symmetry number 12 .

Rotational temperatures (Kelvin)

Rotational constants (GHZ) :

Zero-point vibrational energy

$\begin{array}{lll}79.34349 & 79.34349 & 79.34349\end{array}$

87592.0 (Joules/Mol)

$20.93500 \quad(\mathrm{KCal} / \mathrm{Mol})$

Vibrational temperatures:

1478.80

$1478.80 \quad 1478.80$

(Kelvin)

3138.84

3432.95

3432.95

1597.84

Zero-point correction=

.033362 (Hartree/Particle) 
XXI. Harmonic frequency analysis and zero-point energy for non-deuterated methane-benzene complex with the MP2/6-311++G(d,p) method:

Full mass-weighted force constant matrix:

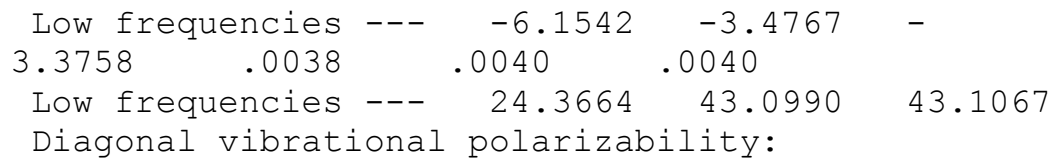

$$
8.0810770 \quad .8708317 \quad .8707219
$$

Harmonic frequencies $(\mathrm{cm} * \star-1)$, IR intensities (KM/Mole), Raman scattering activities (A**4/AMU), depolarization ratios for plane and unpolarized incident light, reduced masses (AMU), force constants (mDyne/A), and normal coordinates:

\begin{tabular}{|c|c|c|c|c|c|c|c|c|c|c|}
\hline & \multicolumn{3}{|c|}{1} & \multicolumn{3}{|c|}{2} & \multicolumn{3}{|c|}{3} \\
\hline & & \multicolumn{3}{|c|}{ A" } & \multicolumn{3}{|c|}{ A" } & \multicolumn{3}{|c|}{$A^{\prime}$} \\
\hline \multicolumn{2}{|c|}{ Frequencies } & -- & \multicolumn{2}{|c|}{24.3372} & \multicolumn{3}{|c|}{43.0961} & \multicolumn{3}{|c|}{43.1041} \\
\hline Red. & masses & -- & \multicolumn{2}{|c|}{1.0208} & \multicolumn{3}{|c|}{2.3034} & \multicolumn{3}{|c|}{2.3029} \\
\hline Frc & consts & -- & \multicolumn{2}{|c|}{.0004} & \multicolumn{3}{|c|}{.0025} & \multicolumn{3}{|c|}{.0025} \\
\hline IR I & nten & -- & \multicolumn{2}{|c|}{.0000} & \multicolumn{3}{|c|}{.0022} & \multicolumn{3}{|c|}{.0022} \\
\hline Atom & AN & $\mathrm{x}$ & $\mathrm{Y}$ & Z & $\mathrm{X}$ & $\mathrm{Y}$ & Z & $\mathrm{x}$ & $\mathrm{Y}$ & $\mathrm{Z}$ \\
\hline 1 & 6 & .00 & .00 & -.01 & .00 & .00 & .03 & .17 & .03 & .00 \\
\hline 2 & 1 & .00 & .00 & -.03 & .00 & .00 & .03 & .30 & .03 & .00 \\
\hline 3 & 6 & .00 & .01 & -.01 & .15 & .00 & .03 & .08 & .03 & .00 \\
\hline 4 & 1 & .00 & .02 & -.01 & .26 & .00 & .03 & .15 & .03 & .00 \\
\hline 5 & 6 & .00 & .01 & .01 & .15 & .00 & .03 & -.08 & .03 & .00 \\
\hline 6 & 1 & .00 & .02 & .01 & .26 & .00 & .03 & -.15 & .03 & .00 \\
\hline 7 & 6 & .00 & .00 & .01 & .00 & .00 & .03 & -.17 & .03 & .00 \\
\hline 8 & 1 & .00 & .00 & .02 & .00 & .00 & .03 & -.30 & .03 & .00 \\
\hline 9 & 6 & .00 & -.01 & .01 & -.15 & .00 & .03 & -.08 & .03 & .00 \\
\hline 10 & 1 & .00 & -.02 & .01 & -.26 & .00 & .03 & -.15 & .03 & .00 \\
\hline 11 & 6 & .00 & -.01 & -.01 & -.15 & .00 & .03 & .08 & .03 & .00 \\
\hline 12 & 1 & .00 & -.02 & -.01 & -.26 & .00 & .03 & .15 & .03 & .00 \\
\hline 13 & 1 & .00 & .00 & .00 & .00 & .00 & .27 & .00 & .27 & .00 \\
\hline 14 & 6 & .00 & .00 & .00 & .00 & .00 & -.16 & .00 & -.16 & .00 \\
\hline 15 & 1 & .00 & .00 & .58 & .00 & .00 & -.31 & -.41 & -.31 & .00 \\
\hline 16 & 1 & .00 & -.50 & -.29 & -.35 & .00 & -.31 & .21 & -.31 & .00 \\
\hline \multirow[t]{2}{*}{17} & 1 & .00 & .50 & -.29 & .36 & .00 & -.31 & .20 & -.31 & .00 \\
\hline & & \multicolumn{3}{|c|}{$A^{\prime}$} & \multicolumn{3}{|c|}{$A^{\prime}$} & & $\begin{array}{r}6 \\
A^{\prime \prime}\end{array}$ & \\
\hline Freq & dencies & -- & 84.557 & & & 109.462 & & & 109.4688 & \\
\hline Red. & masses & -- & 3.518 & & & 1.208 & & & 1.2079 & \\
\hline Frc & consts & -- & .014 & & & .008 & & & .0085 & \\
\hline IR I & nten & -- & .035 & & & .197 & & & .1978 & \\
\hline Atom & AN & X & Y & Z & $\mathrm{x}$ & Y & Z & $\mathrm{x}$ & Y & Z \\
\hline 1 & 6 & .09 & .00 & .00 & .05 & .02 & .00 & .00 & .00 & .02 \\
\hline 2 & 1 & .09 & .00 & .00 & .10 & .02 & .00 & .00 & .00 & .02 \\
\hline 3 & 6 & .09 & .00 & .00 & .03 & .02 & .00 & .05 & .00 & .02 \\
\hline 4 & 1 & .09 & .00 & .00 & .05 & .02 & .00 & .08 & .00 & .02 \\
\hline 5 & 6 & .09 & .00 & .00 & -.03 & .02 & .00 & .05 & .00 & .02 \\
\hline 6 & 1 & .09 & .00 & .00 & -.05 & .02 & .00 & .08 & .00 & .02 \\
\hline 7 & 6 & .09 & .00 & .00 & -.05 & .02 & .00 & .00 & .00 & .02 \\
\hline 8 & 1 & .09 & .00 & .00 & -.10 & .02 & .00 & .00 & .00 & .02 \\
\hline 9 & 6 & .09 & .00 & .00 & -.03 & .02 & .00 & -.05 & .00 & .02 \\
\hline 10 & 1 & .09 & .00 & .00 & -.05 & .02 & .00 & -.08 & .00 & .02 \\
\hline 11 & 6 & .09 & .00 & .00 & .03 & .02 & .00 & -.05 & .00 & .02 \\
\hline 12 & 1 & .09 & .00 & .00 & .05 & .02 & .00 & -.08 & .00 & .02 \\
\hline 13 & 1 & -.42 & .00 & .00 & .00 & -.67 & .00 & .00 & .00 & -.67 \\
\hline
\end{tabular}




\begin{tabular}{|c|c|c|c|c|c|c|c|c|c|c|}
\hline 14 & 6 & -.43 & .00 & .00 & .00 & -.09 & .00 & .00 & .00 & -.09 \\
\hline 15 & 1 & -.43 & .00 & .00 & .56 & .11 & .00 & .00 & .00 & .11 \\
\hline 16 & 1 & -.43 & .00 & .00 & -.28 & .11 & .00 & .48 & .00 & .11 \\
\hline \multirow[t]{2}{*}{17} & 1 & -.43 & .00 & .00 & -.28 & $\dot{8}^{11}$ & .00 & -.49 & $\dot{9}^{.00}$ & .11 \\
\hline & & \multicolumn{3}{|c|}{$A^{\prime}$} & \multicolumn{3}{|c|}{$A^{\prime \prime}$} & \multicolumn{3}{|c|}{$A^{\prime}$} \\
\hline \multicolumn{2}{|c|}{ Frequencies } & -- & 369.6281 & & \multicolumn{3}{|c|}{369.6293} & \multicolumn{3}{|c|}{469.8032} \\
\hline Red. & masses & -- & 3.4123 & & \multicolumn{3}{|c|}{3.4124} & \multicolumn{3}{|c|}{9.9359} \\
\hline \multicolumn{2}{|c|}{ Frc consts } & -- & .2747 & & \multicolumn{3}{|c|}{.2747} & \multicolumn{3}{|c|}{1.2921} \\
\hline \multicolumn{2}{|c|}{ IR Inten } & -- & .0003 & & \multicolumn{3}{|c|}{.0003} & \multicolumn{3}{|c|}{.0000} \\
\hline Atom & AN & $\mathrm{X}$ & $\mathrm{Y}$ & Z & $\mathrm{X}$ & Y & Z & $\mathrm{X}$ & $\mathrm{Y}$ & Z \\
\hline 1 & 6 & .27 & .00 & .00 & .00 & .00 & .00 & .37 & .00 & .00 \\
\hline 2 & 1 & .51 & .00 & .00 & .00 & .00 & .00 & .18 & .00 & .00 \\
\hline 3 & 6 & -.13 & .00 & .00 & .23 & .00 & .00 & -.37 & .00 & .00 \\
\hline 4 & 1 & -.26 & .00 & .00 & .44 & .00 & .00 & -.18 & .00 & .00 \\
\hline 5 & 6 & -.14 & .00 & .00 & -.23 & .00 & .00 & .37 & .00 & .00 \\
\hline 6 & 1 & -.25 & .00 & .00 & -.44 & .00 & .00 & .18 & .00 & .00 \\
\hline 7 & 6 & .27 & .00 & .00 & .00 & .00 & .00 & -.37 & .00 & .00 \\
\hline 8 & 1 & .51 & .00 & .00 & .00 & .00 & .00 & -.18 & .00 & .00 \\
\hline 9 & 6 & -.13 & .00 & .00 & .23 & .00 & .00 & .37 & .00 & .00 \\
\hline 10 & 1 & -.25 & .00 & .00 & .44 & .00 & .00 & .18 & .00 & .00 \\
\hline 11 & 6 & -.14 & .00 & .00 & -.23 & .00 & .00 & -.37 & .00 & .00 \\
\hline 12 & 1 & -.26 & .00 & .00 & -.44 & .00 & .00 & -.18 & .00 & .00 \\
\hline 13 & 1 & .00 & .00 & .00 & .00 & .00 & .00 & .00 & .00 & .00 \\
\hline 14 & 6 & .00 & .00 & .00 & .00 & .00 & .00 & .00 & .00 & .00 \\
\hline 15 & 1 & .00 & .00 & .00 & .00 & .00 & .00 & .00 & .00 & .00 \\
\hline 16 & 1 & .00 & .00 & .00 & .00 & .00 & .00 & .00 & .00 & .00 \\
\hline \multirow[t]{3}{*}{17} & 1 & .00 & .00 & .00 & .00 & .00 & .00 & .00 & .00 & .00 \\
\hline & & & 10 & & & 11 & & & 12 & \\
\hline & & & ?A & & & ?A & & & $A^{\prime}$ & \\
\hline Frequ & dencies & -- & 608.7810 & & & 608.7815 & & & 666.6115 & \\
\hline Red. & masses & -- & 6.0129 & & & 6.0129 & & & 1.0850 & \\
\hline Frc $C$ & yonsts & -- & 1.3130 & & & 1.3130 & & & .2841 & \\
\hline IR In & iten & -- & .0000 & & & .0000 & & & 127.8308 & \\
\hline Atom & AN & $\mathrm{X}$ & Y & Z & $\mathrm{X}$ & Y & Z & $\mathrm{X}$ & $\mathrm{Y}$ & Z \\
\hline 1 & 6 & .00 & .00 & .15 & .00 & .36 & .00 & -.03 & .00 & .00 \\
\hline 2 & 1 & .00 & .00 & -.23 & -.01 & .36 & .00 & .41 & .00 & .00 \\
\hline 3 & 6 & .00 & .22 & .23 & .00 & .03 & -.22 & -.03 & .00 & .00 \\
\hline 4 & 1 & -.01 & .05 & .33 & .00 & -.26 & -.05 & .40 & .00 & .00 \\
\hline 5 & 6 & .00 & .22 & -.23 & .00 & -.03 & -.22 & -.03 & .00 & .00 \\
\hline 6 & 1 & .01 & .05 & -.33 & .00 & .26 & -.05 & .41 & .00 & .00 \\
\hline 7 & 6 & .00 & .00 & -.15 & .00 & -.36 & .00 & -.03 & .00 & .00 \\
\hline 8 & 1 & .00 & .00 & .23 & -.01 & -.36 & .00 & .40 & .00 & .00 \\
\hline 9 & 6 & .00 & -.22 & -.23 & .00 & -.03 & .22 & -.03 & .00 & .00 \\
\hline 10 & 1 & -.01 & -.05 & -.33 & .00 & .26 & .05 & .41 & .00 & .00 \\
\hline 11 & 6 & .00 & -.22 & .23 & .00 & .03 & .22 & -.03 & .00 & .00 \\
\hline 12 & 1 & .01 & -.05 & .33 & .00 & -.26 & .05 & .40 & .00 & .00 \\
\hline 13 & 1 & .00 & .00 & .00 & .00 & .00 & .00 & .00 & .00 & .00 \\
\hline 14 & 6 & .00 & .00 & .00 & .00 & .00 & .00 & .00 & .00 & .00 \\
\hline 15 & 1 & .00 & .00 & .00 & .00 & .00 & .00 & .00 & .00 & .00 \\
\hline 16 & 1 & .00 & .00 & .00 & .00 & .00 & .00 & .00 & .00 & .00 \\
\hline 17 & 1 & .00 & .00 & .00 & .00 & .00 & .00 & .00 & .00 & .00 \\
\hline & & & 13 & & & 14 & & & 15 & \\
\hline & & & $A^{\prime}$ & & & $A^{\prime \prime}$ & & & $A^{\prime}$ & \\
\hline Frequ & dencies & -- & 834.3453 & & & 834.3542 & & & 897.4803 & \\
\hline Red. & masses & -- & 1.2494 & & & 1.2494 & & & 1.2780 & \\
\hline Frc $C$ & yonsts & -- & .5124 & & & .5124 & & & .6065 & \\
\hline
\end{tabular}




\begin{tabular}{|c|c|c|c|c|c|c|c|c|c|c|}
\hline IR I & nten & -- & .131 & & & .131 & & & .0013 & \\
\hline Atom & AN & $\mathrm{x}$ & $\mathrm{Y}$ & Z & $\mathrm{X}$ & $\mathrm{Y}$ & Z & $\mathrm{x}$ & $\mathrm{Y}$ & Z \\
\hline 1 & 6 & -.09 & .00 & .00 & .00 & .00 & .00 & -.09 & .00 & .00 \\
\hline 2 & 1 & .60 & .00 & .00 & .00 & .00 & .00 & .54 & .00 & .00 \\
\hline 3 & 6 & -.04 & .00 & .00 & .07 & .00 & .00 & .05 & .00 & .00 \\
\hline 4 & 1 & .27 & .00 & .00 & -.47 & .00 & .00 & -.30 & .00 & .00 \\
\hline 5 & 6 & .04 & .00 & .00 & .08 & .00 & .00 & .04 & .00 & .00 \\
\hline 6 & 1 & -.30 & .00 & .00 & -.52 & .00 & .00 & -.27 & .00 & .00 \\
\hline 7 & 6 & .08 & .00 & .00 & .00 & .00 & .00 & -.09 & .00 & .00 \\
\hline 8 & 1 & -.54 & .00 & .00 & .00 & .00 & .00 & .60 & .00 & .00 \\
\hline 9 & 6 & .04 & .00 & .00 & -.08 & .00 & .00 & .04 & .00 & .00 \\
\hline 10 & 1 & -.30 & .00 & .00 & .52 & .00 & .00 & -.27 & .00 & .00 \\
\hline 11 & 6 & -.04 & .00 & .00 & -.07 & .00 & .00 & .05 & .00 & .00 \\
\hline 12 & 1 & .27 & .00 & .00 & .47 & .00 & .00 & -.30 & .00 & .00 \\
\hline 13 & 1 & .00 & .00 & .00 & .00 & .00 & .00 & .00 & .00 & .00 \\
\hline 14 & 6 & .00 & .00 & .00 & .00 & .00 & .00 & .00 & .00 & .00 \\
\hline 15 & 1 & .00 & .00 & .00 & .00 & .00 & .00 & .00 & .00 & .00 \\
\hline 16 & 1 & .00 & .00 & .00 & .00 & .00 & .00 & .00 & .00 & .00 \\
\hline 17 & 1 & .00 & .00 & .00 & .00 & .00 & .00 & .00 & .00 & .00 \\
\hline & & & 16 & & & 17 & & & 18 & \\
\hline & & & A" & & & $A^{\prime}$ & & & $A^{\prime}$ & \\
\hline Freq & uencies & -- & 897.488 & & & .7 .656 & & & 008.5332 & \\
\hline Red. & masses & -- & 1.278 & & & 1.025 & & & 5.9699 & \\
\hline Frc & consts & -- & .606 & & & .520 & & & 3.5776 & \\
\hline IR I & nten & -- & .001 & & & .003 & & & .0335 & \\
\hline Atom & AN & $\mathrm{x}$ & $\mathrm{Y}$ & Z & $\mathrm{x}$ & $\mathrm{Y}$ & Z & $\mathrm{x}$ & $\mathrm{Y}$ & Z \\
\hline 1 & 6 & .00 & .00 & .00 & .02 & .00 & .00 & .00 & .27 & .00 \\
\hline 2 & 1 & .00 & .00 & .00 & -.41 & .00 & .00 & .00 & .30 & .00 \\
\hline 3 & 6 & -.08 & .00 & .00 & -.02 & .00 & .00 & .00 & .14 & .24 \\
\hline 4 & 1 & .52 & .00 & .00 & .41 & .00 & .00 & .00 & .15 & .26 \\
\hline 5 & 6 & .07 & .00 & .00 & .02 & .00 & .00 & .00 & -.14 & .24 \\
\hline 6 & 1 & -.47 & .00 & .00 & -.41 & .00 & .00 & .00 & -.15 & .26 \\
\hline 7 & 6 & .00 & .00 & .00 & -.02 & .00 & .00 & .00 & -.28 & .00 \\
\hline 8 & 1 & .00 & .00 & .00 & .41 & .00 & .00 & .00 & -.30 & .00 \\
\hline 9 & 6 & -.07 & .00 & .00 & .02 & .00 & .00 & .00 & -.14 & -.24 \\
\hline 10 & 1 & .47 & .00 & .00 & -.41 & .00 & .00 & .00 & -.15 & -.26 \\
\hline 11 & 6 & .08 & .00 & .00 & -.02 & .00 & .00 & .00 & .14 & -.24 \\
\hline 12 & 1 & -.52 & .00 & .00 & .41 & .00 & .00 & .00 & .15 & -.26 \\
\hline 13 & 1 & .00 & .00 & .00 & .00 & .00 & .00 & .00 & .00 & .00 \\
\hline 14 & 6 & .00 & .00 & .00 & .00 & .00 & .00 & .00 & .00 & .00 \\
\hline 15 & 1 & .00 & .00 & .00 & .00 & .00 & .00 & .00 & .00 & .00 \\
\hline 16 & 1 & .00 & .00 & .00 & .00 & .00 & .00 & .00 & .00 & .00 \\
\hline 17 & 1 & .00 & .00 & .00 & .00 & .00 & .00 & .00 & .00 & .00 \\
\hline & & & 19 & & & 20 & & & 21 & \\
\hline & & & $A^{\prime}$ & & & A" & & & $A^{\prime}$ & \\
\hline Freq & uencies & -- & 1011.172 & & & 9.445 & & & 059.4503 & \\
\hline Red. & masses & -- & 6.536 & & & 1.698 & & & 1.6984 & \\
\hline Frc & consts & -- & 3.937 & & & 1.123 & & & 1.1232 & \\
\hline IR I & nten & -- & .000 & & & 4.531 & & & 4.5311 & \\
\hline Atom & AN & $\mathrm{x}$ & Y & Z & $\mathrm{X}$ & Y & Z & $\mathrm{X}$ & Y & Z \\
\hline 1 & 6 & .00 & .29 & .00 & .00 & .00 & .08 & .00 & -.12 & .00 \\
\hline 2 & 1 & .00 & .29 & .00 & .00 & .00 & .54 & .00 & -.13 & .00 \\
\hline 3 & 6 & .00 & -.14 & -.25 & .00 & -.09 & -.07 & .00 & .03 & -.09 \\
\hline 4 & 1 & .00 & -.14 & -.25 & .00 & -.29 & .04 & .00 & .38 & -.29 \\
\hline 5 & 6 & .00 & -.15 & .25 & .00 & .09 & -.07 & .00 & .03 & .09 \\
\hline 6 & 1 & .00 & -.15 & .25 & .00 & .29 & .04 & .00 & .38 & .29 \\
\hline 7 & 6 & .00 & .29 & .00 & .00 & .00 & .08 & .00 & -.12 & .00 \\
\hline
\end{tabular}




\begin{tabular}{|c|c|c|c|c|c|c|c|c|c|c|}
\hline 8 & 1 & .00 & .29 & .00 & .00 & .00 & .54 & .00 & -.13 & .00 \\
\hline 9 & 6 & .00 & -.15 & -.25 & .00 & -.09 & -.07 & .00 & .03 & -.09 \\
\hline 10 & 1 & .00 & -.15 & -.25 & .00 & -.29 & .04 & .00 & .38 & -.29 \\
\hline 11 & 6 & .00 & -.14 & .25 & .00 & .09 & -.07 & .00 & .03 & .09 \\
\hline 12 & 1 & .00 & -.14 & .25 & .00 & .29 & .04 & .00 & .38 & .29 \\
\hline 13 & 1 & .00 & .00 & .00 & .00 & .00 & .00 & .00 & .00 & .00 \\
\hline 14 & 6 & .00 & .00 & .00 & .00 & .00 & .00 & .00 & .00 & .00 \\
\hline 15 & 1 & .00 & .00 & .00 & .00 & .00 & .00 & .00 & .00 & .00 \\
\hline 16 & 1 & .00 & .00 & .00 & .00 & .00 & .00 & .00 & .00 & .00 \\
\hline \multirow[t]{3}{*}{17} & 1 & .00 & .00 & .00 & .00 & .00 & .00 & .00 & .00 & .00 \\
\hline & & & 22 & & & 23 & & & 24 & \\
\hline & & & $\mathrm{A}^{\prime \prime}$ & & & A" & & \multirow{2}{*}{\multicolumn{3}{|c|}{1198.2932}} \\
\hline \multicolumn{2}{|c|}{ Frequencies } & -- & \multicolumn{2}{|c|}{1173.5073} & \multicolumn{3}{|c|}{1198.2899} & & & \\
\hline \multicolumn{2}{|c|}{ Red. masses } & -- & \multicolumn{2}{|l|}{1.0313} & \multicolumn{3}{|c|}{1.1288} & \multicolumn{3}{|c|}{1.1288} \\
\hline \multirow{2}{*}{\multicolumn{2}{|c|}{$\begin{array}{l}\text { Frc consts } \\
\text { IR Inten }\end{array}$}} & -- & \multicolumn{2}{|l|}{$\begin{array}{r}.8368 \\
0000\end{array}$} & \multicolumn{3}{|c|}{.9549} & \multicolumn{3}{|c|}{.9549} \\
\hline & & -- & \multicolumn{2}{|l|}{.0000} & & .000 & & & .0000 & \\
\hline Atom & AN & $\mathrm{x}$ & Y & Z & $\mathrm{x}$ & Y & Z & $\mathrm{x}$ & $\mathrm{Y}$ & Z \\
\hline 1 & 6 & .00 & .00 & .02 & .00 & .00 & .06 & .00 & .01 & .00 \\
\hline 2 & 1 & .00 & .00 & .41 & .00 & .00 & .57 & .00 & .00 & .00 \\
\hline 3 & 6 & .00 & .02 & -.01 & .00 & .03 & -.01 & .00 & .04 & -.03 \\
\hline 4 & 1 & .00 & .35 & -.20 & .00 & .25 & -.14 & .00 & .43 & -.25 \\
\hline 5 & 6 & .00 & -.02 & -.01 & .00 & .03 & .01 & .00 & -.04 & -.0 \\
\hline 6 & 1 & .00 & -.35 & -.20 & .00 & .25 & .14 & .00 & -.43 & -.2 \\
\hline 7 & 6 & .00 & .00 & .02 & .00 & .00 & -.06 & .00 & -.01 & .0 \\
\hline 8 & 1 & .00 & .00 & .41 & .00 & .00 & -.57 & .00 & .00 & .00 \\
\hline 9 & 6 & .00 & .02 & -.01 & .00 & -.03 & .01 & .00 & -.04 & .03 \\
\hline 10 & 1 & .00 & .35 & -.20 & .00 & -.25 & .14 & .00 & -.43 & .2 \\
\hline 11 & 6 & .00 & -.02 & -.01 & .00 & -.03 & -.01 & .00 & .04 & .0 \\
\hline 12 & 1 & .00 & -.35 & -.20 & .00 & -.25 & -.14 & .00 & .43 & .2 \\
\hline 13 & 1 & .00 & .00 & .00 & .00 & .00 & .00 & .00 & .00 & .0 \\
\hline 14 & 6 & .00 & .00 & .00 & .00 & .00 & .00 & .00 & .00 & .00 \\
\hline 15 & 1 & .00 & .00 & .00 & .00 & .00 & .00 & .00 & .00 & .00 \\
\hline 16 & 1 & .00 & .00 & .00 & .00 & .00 & .00 & .00 & .00 & .0 \\
\hline 17 & 1 & .00 & .00 & .00 & .00 & .00 & .00 & .00 & .00 & .0 \\
\hline & & & 25 & & & 26 & & & 27 & \\
\hline & & & $A^{\prime}$ & & & A" & & & $A^{\prime}$ & \\
\hline Frequ & encies & -- & 1353.7761 & & & 61.710 & & & 61.7108 & \\
\hline Red. & masses & -- & 1.1784 & & & 1.178 & & & 1.1785 & \\
\hline Frc & onsts & -- & 1.2725 & & & 1.287 & & & 1.2875 & \\
\hline IR In & ten & -- & 11.8969 & & & 4.005 & & & 4.0056 & \\
\hline Atom & AN & $\mathrm{x}$ & $\mathrm{Y}$ & Z & $\mathrm{x}$ & $\mathrm{Y}$ & Z & $\mathrm{x}$ & $\mathrm{Y}$ & Z \\
\hline 1 & 6 & .00 & .00 & .00 & .00 & .00 & .00 & .00 & .00 & .00 \\
\hline 2 & 1 & .00 & .00 & .00 & .00 & .00 & .01 & .00 & .00 & .0 \\
\hline 3 & 6 & .00 & .00 & .00 & .00 & .00 & .00 & .00 & .00 & .0 \\
\hline 4 & 1 & .00 & .00 & .00 & .00 & .00 & .00 & .00 & .00 & .0 \\
\hline 5 & 6 & .00 & .00 & .00 & .00 & .00 & .00 & .00 & .00 & .0 \\
\hline 6 & 1 & .00 & .00 & .00 & .00 & .00 & .00 & .00 & .01 & .0 \\
\hline 7 & 6 & .00 & .00 & .00 & .00 & .00 & .00 & .00 & .00 & .0 \\
\hline 8 & 1 & .00 & .00 & .00 & .00 & .00 & .01 & .00 & .00 & .0 \\
\hline 9 & 6 & .00 & .00 & .00 & .00 & .00 & .00 & .00 & .00 & .0 \\
\hline 10 & 1 & .00 & .00 & .00 & .00 & .00 & .00 & .00 & .01 & .0 \\
\hline 11 & 6 & .00 & .00 & .00 & .00 & .00 & .00 & .00 & .00 & .0 \\
\hline 12 & 1 & .00 & .00 & .00 & .00 & .00 & .00 & .00 & .00 & .0 \\
\hline 13 & 1 & .10 & .00 & .00 & .00 & .00 & .61 & .00 & .61 & .0 \\
\hline 14 & 6 & .12 & .00 & .00 & .00 & .00 & -.12 & .00 & -.12 & .0 \\
\hline 15 & 1 & -.53 & -.22 & .00 & .00 & .00 & .61 & .21 & -.02 & .0 \\
\hline 16 & 1 & -.53 & .11 & -.19 & .18 & .27 & .14 & -.10 & .45 & .2 \\
\hline
\end{tabular}




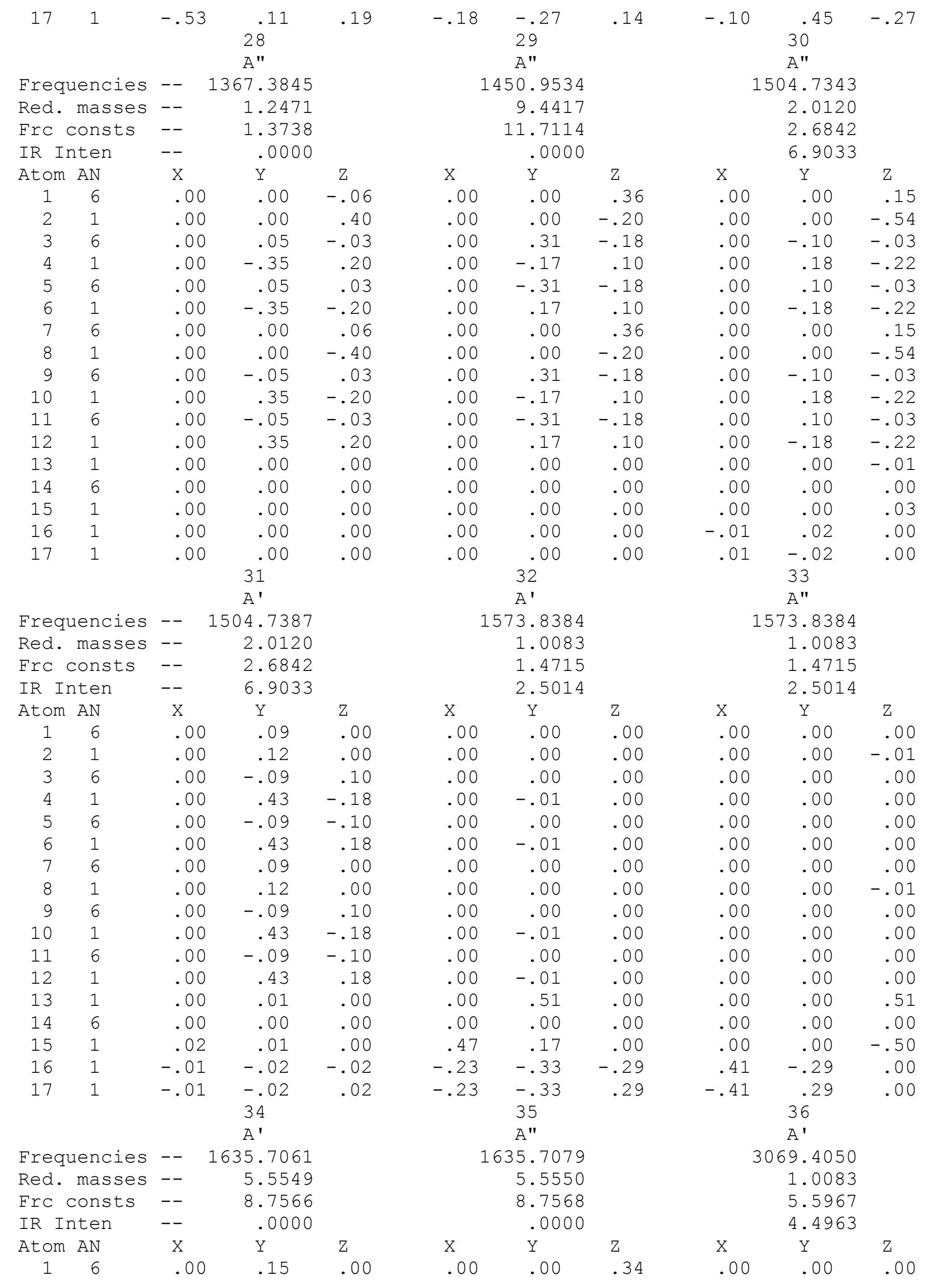




\begin{tabular}{|c|c|c|c|c|c|c|c|c|c|c|}
\hline 2 & 1 & .00 & .17 & .00 & .00 & .00 & -.41 & .00 & .00 & .00 \\
\hline 3 & 6 & .00 & -.29 & .08 & .00 & .08 & -.20 & .00 & .00 & .00 \\
\hline 4 & 1 & .00 & .26 & -.25 & .00 & -.25 & -.03 & .00 & .00 & .00 \\
\hline 5 & 6 & .00 & .29 & .08 & .00 & .08 & .20 & .00 & .00 & .00 \\
\hline 6 & 1 & .00 & -.26 & -.25 & .00 & -.25 & .03 & .00 & .00 & .00 \\
\hline 7 & 6 & .00 & -.15 & .00 & .00 & .00 & -.34 & .00 & .00 & .00 \\
\hline 8 & 1 & .00 & -.17 & .00 & .00 & .00 & .41 & .00 & .00 & .00 \\
\hline 9 & 6 & .00 & .29 & -.08 & .00 & -.08 & .20 & .00 & .00 & .00 \\
\hline 10 & 1 & .00 & -.26 & .25 & .00 & .25 & .03 & .00 & .00 & .00 \\
\hline 11 & 6 & .00 & -.29 & -.08 & .00 & -.08 & -.20 & .00 & .00 & .0 \\
\hline 12 & 1 & .00 & .26 & .25 & .00 & .25 & -.03 & .00 & .00 & .0 \\
\hline 13 & 1 & .00 & .00 & .00 & .00 & .00 & .00 & .44 & .00 & .0 \\
\hline 14 & 6 & .00 & .00 & .00 & .00 & .00 & .00 & .01 & .00 & .0 \\
\hline 15 & 1 & .00 & .00 & .00 & .00 & .00 & .00 & -.17 & .49 & .0 \\
\hline 16 & 1 & .00 & .00 & .00 & .00 & .00 & .00 & -.17 & -.24 & .4 \\
\hline \multirow[t]{3}{*}{17} & 1 & .00 & .00 & .00 & .00 & .00 & .00 & -.17 & -.24 & -.4 \\
\hline & & & 37 & & & 38 & & & 39 & \\
\hline & & & $A^{\prime}$ & & & A" & & & $A^{\prime}$ & \\
\hline \multicolumn{2}{|c|}{ Frequencies } & -- & \multicolumn{2}{|l|}{3195.5719} & \multicolumn{3}{|c|}{3200.8193} & \multicolumn{3}{|c|}{3200.8226} \\
\hline Red. & masses & -- & 1.0839 & & \multicolumn{3}{|c|}{1.1012} & \multicolumn{3}{|c|}{1.1012} \\
\hline \multicolumn{2}{|c|}{ Frc consts } & -- & 6.5215 & & \multicolumn{3}{|c|}{6.6474} & \multicolumn{3}{|c|}{6.6475} \\
\hline \multicolumn{2}{|c|}{ IR Inten } & -- & .0000 & & \multicolumn{3}{|c|}{17.3286} & \multicolumn{3}{|c|}{17.3290} \\
\hline Atom & AN & $\mathrm{X}$ & $\mathrm{Y}$ & Z & $\mathrm{x}$ & Y & Z & $\mathrm{x}$ & $\mathrm{Y}$ & Z \\
\hline 1 & 6 & .00 & -.03 & .00 & .00 & .00 & .00 & .00 & .00 & .00 \\
\hline 2 & 1 & .00 & .41 & .00 & .00 & .00 & .00 & .00 & .01 & .0 \\
\hline 3 & 6 & .00 & .02 & .03 & .00 & .00 & .00 & .00 & .00 & .0 \\
\hline 4 & 1 & .00 & -.20 & -.35 & .00 & .00 & .00 & .00 & .00 & .0 \\
\hline 5 & 6 & .00 & .02 & -.03 & .00 & .00 & .00 & .00 & .00 & .0 \\
\hline 6 & 1 & .00 & -.20 & .35 & .00 & -.01 & .01 & .00 & .00 & -.0 \\
\hline 7 & 6 & .00 & -.03 & .00 & .00 & .00 & .00 & .00 & .00 & .0 \\
\hline 8 & 1 & .00 & .41 & .00 & .00 & .00 & .00 & .00 & .00 & .0 \\
\hline 9 & 6 & .00 & .02 & .03 & .00 & .00 & .00 & .00 & .00 & .00 \\
\hline 10 & 1 & .00 & -.20 & -.35 & .00 & .01 & .01 & .00 & .00 & .0 \\
\hline 11 & 6 & .00 & .02 & -.03 & .00 & .00 & .00 & .00 & .00 & .0 \\
\hline 12 & 1 & .00 & -.20 & .35 & .00 & .00 & .00 & .00 & .00 & .0 \\
\hline 13 & 1 & .00 & .00 & .00 & .00 & .00 & .02 & .00 & .02 & .00 \\
\hline 14 & 6 & .00 & .00 & .00 & .00 & .00 & .09 & .00 & .09 & .0 \\
\hline 15 & 1 & .00 & .00 & .00 & .00 & .00 & .02 & .28 & -.76 & .0 \\
\hline 16 & 1 & .00 & .00 & .00 & .24 & .34 & -.57 & -.14 & -.18 & .3 \\
\hline \multirow[t]{3}{*}{17} & 1 & .00 & .00 & .00 & -.24 & -.34 & -.57 & -.14 & -.18 & -.3 \\
\hline & & & 40 & & & 41 & & & 42 & \\
\hline & & & A" & & & $A^{\prime}$ & & & $\mathrm{A}^{\prime \prime}$ & \\
\hline \multirow{2}{*}{\multicolumn{2}{|c|}{$\begin{array}{l}\text { Frequencies } \\
\text { Red. masses }\end{array}$}} & -- & 3206.1043 & & & 206.1232 & & & 221.9830 & \\
\hline & & -- & 1.0883 & & & 1.0883 & & & 1.0964 & \\
\hline Frc & onsts & -- & 6.5908 & & & 6.5909 & & & 6.7058 & \\
\hline IR Ir & ten & -- & .0035 & & & .0035 & & & 26.1254 & \\
\hline Atom & AN & X & $\mathrm{Y}$ & Z & $\mathrm{x}$ & Y & Z & $\mathrm{x}$ & Y & Z \\
\hline 1 & 6 & .00 & .00 & .00 & .00 & -.05 & .00 & .00 & .00 & .00 \\
\hline 2 & 1 & .00 & .00 & .00 & .00 & .58 & .00 & .00 & .00 & .0 \\
\hline 3 & 6 & .00 & .02 & .04 & .00 & .01 & .02 & .00 & -.02 & -.0 \\
\hline 4 & 1 & .00 & -.25 & -.43 & .00 & -.15 & -.25 & .00 & .25 & .4 \\
\hline 5 & 6 & .00 & .02 & -.04 & .00 & -.01 & .02 & .00 & .02 & -.0 \\
\hline 6 & 1 & .00 & -.25 & .43 & .00 & .15 & -.25 & .00 & -.25 & .4 \\
\hline 7 & 6 & .00 & .00 & .00 & .00 & .05 & .00 & .00 & .00 & .0 \\
\hline 8 & 1 & .00 & .00 & .00 & .00 & -.57 & .00 & .00 & .00 & .0 \\
\hline 9 & 6 & .00 & -.02 & -.04 & .00 & -.01 & -.02 & .00 & -.02 & -.0 \\
\hline 10 & 1 & .00 & .25 & .43 & .00 & .15 & .25 & .00 & .25 & .4 \\
\hline
\end{tabular}




\begin{tabular}{|c|c|c|c|c|c|c|c|c|c|c|}
\hline 11 & 6 & .00 & -.02 & .04 & .00 & .01 & -.02 & .00 & .02 & -.04 \\
\hline 12 & 1 & .00 & .25 & -.43 & .00 & -.15 & .25 & .00 & -.25 & .43 \\
\hline 13 & 1 & .00 & .00 & .00 & .00 & .00 & .00 & .00 & .00 & .00 \\
\hline 14 & 6 & .00 & .00 & .00 & .00 & .00 & .00 & .00 & .00 & .00 \\
\hline 15 & 1 & .00 & .00 & .00 & .00 & .01 & .00 & .00 & .00 & .00 \\
\hline 16 & 1 & .00 & .00 & .01 & .00 & .00 & .00 & .00 & .00 & .01 \\
\hline 17 & 1 & .00 & .00 & .01 & .00 & .00 & .00 & .00 & .00 & .01 \\
\hline & & & 43 & & & 44 & & & 45 & \\
\hline & & & $A^{\prime}$ & & & $A^{\prime}$ & & & $A^{\prime}$ & \\
\hline \multicolumn{2}{|c|}{ Frequencies } & -- & 3222.0020 & & \multicolumn{3}{|c|}{3222.3328} & \multicolumn{3}{|c|}{3231.5209} \\
\hline \multicolumn{2}{|c|}{ Red. masses } & -- & 1.0964 & & \multicolumn{3}{|c|}{1.1005} & \multicolumn{3}{|c|}{1.1012} \\
\hline \multicolumn{2}{|c|}{ Frc consts } & -- & 6.7059 & & \multicolumn{3}{|c|}{6.7326} & \multicolumn{3}{|c|}{6.7756} \\
\hline \multicolumn{2}{|c|}{ IR Inten } & -- & 26.1243 & & \multicolumn{3}{|c|}{6.0508} & \multicolumn{3}{|c|}{.1638} \\
\hline Atom & AN & $\mathrm{x}$ & $\mathrm{Y}$ & Z & $\mathrm{x}$ & $\mathrm{Y}$ & Z & $\mathrm{x}$ & $\mathrm{Y}$ & Z \\
\hline 1 & 6 & .00 & -.05 & .00 & .00 & .00 & .00 & .00 & -.04 & .00 \\
\hline 2 & 1 & .00 & .57 & .00 & .00 & -.01 & .00 & .00 & .41 & .00 \\
\hline 3 & 6 & .00 & -.01 & -.02 & .00 & .00 & .00 & .00 & -.02 & -.03 \\
\hline 4 & 1 & .00 & .14 & .25 & .00 & -.01 & -.01 & .00 & .20 & .35 \\
\hline 5 & 6 & .00 & -.01 & .02 & .00 & .00 & .00 & .00 & .02 & -.03 \\
\hline 6 & 1 & .00 & .14 & -.25 & .00 & .01 & -.01 & .00 & -.20 & .35 \\
\hline 7 & 6 & .00 & -.05 & .00 & .00 & .00 & .00 & .00 & .04 & .00 \\
\hline 8 & 1 & .00 & .58 & .00 & .00 & .01 & .00 & .00 & -.41 & .00 \\
\hline 9 & 6 & .00 & -.01 & -.02 & .00 & .00 & .00 & .00 & .02 & .03 \\
\hline 10 & 1 & .00 & .14 & .25 & .00 & .01 & .01 & .00 & -.20 & -.35 \\
\hline 11 & 6 & .00 & -.01 & .02 & .00 & .00 & .00 & .00 & -.02 & .03 \\
\hline 12 & 1 & .00 & .14 & -.25 & .00 & -.01 & .01 & .00 & .20 & -.35 \\
\hline 13 & 1 & .00 & .00 & .00 & .90 & .00 & .00 & .03 & .00 & .00 \\
\hline 14 & 6 & .00 & .00 & .00 & -.09 & .00 & .00 & .00 & .00 & .00 \\
\hline 15 & 1 & .00 & .01 & .00 & .07 & -.24 & .00 & .00 & -.01 & .00 \\
\hline 16 & 1 & .00 & .00 & .00 & .07 & .12 & -.21 & .00 & .00 & -.01 \\
\hline 17 & 1 & .00 & .00 & .00 & .07 & .12 & .21 & .00 & .00 & .01 \\
\hline
\end{tabular}

- Thermochemistry -

Temperature 298.150 Kelvin. Pressure 1.00000 Atm.

Atom 1 has atomic number 6 and mass 12.00000

Atom 2 has atomic number 1 and mass 1.00783

Atom 3 has atomic number 6 and mass 12.00000

Atom 4 has atomic number 1 and mass 1.00783

Atom 5 has atomic number 6 and mass 12.00000

Atom 6 has atomic number 1 and mass 1.00783

Atom 7 has atomic number 6 and mass 12.00000

Atom 8 has atomic number 1 and mass 1.00783

Atom 9 has atomic number 6 and mass 12.00000

Atom 10 has atomic number 1 and mass 1.00783

Atom 11 has atomic number 6 and mass 12.00000

Atom 12 has atomic number 1 and mass 1.00783

Atom 13 has atomic number 1 and mass 1.00783

Atom 14 has atomic number 6 and mass 12.00000

Atom 15 has atomic number 1 and mass 1.00783

Atom 16 has atomic number 1 and mass 1.00783

Atom 17 has atomic number 1 and mass 1.00783

Molecular mass: 94.07825 amu.

Principal axes and moments of inertia in atomic units:

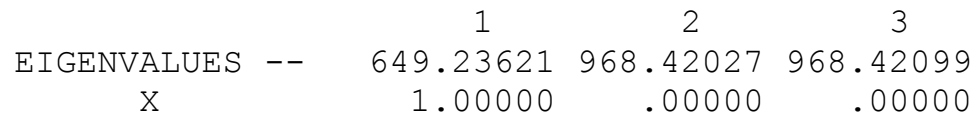




$\begin{array}{lrrr}Y & .00000 & 1.00000 & .00000 \\ Z & .00000 & .00000 & 1.00000\end{array}$

This molecule is an asymmetric top.

Rotational symmetry number 1 .

Warning -- assumption of classical behavior for rotation may cause significant error

$\begin{array}{llll}\text { Rotational temperatures (Kelvin) } & .13341 & .08944 & .08944\end{array}$

$\begin{array}{llll}\text { Rotational constants (GHZ) : } & 2.77979 & 1.86359 & 1.86359\end{array}$

Zero-point vibrational energy 382255.1 (Joules/Mol)

91.36116 (Kcal/Mol)

Warning -- explicit consideration of 11 degrees of freedom as vibrations may cause significant error (Kelvin)

$\begin{array}{rrrr}157.50 & 531.81 & 531.81 & 675.94\end{array}$$$
\begin{array}{lllll}
875.90 & 959.10 & 1200.44 & 1200.45 & 1291.27
\end{array}
$$$$
\begin{array}{lllll}
1291.28 & 1334.69 & 1451.05 & 1454.85 & 1524.30
\end{array}
$$$$
\begin{array}{llllll}
1524.31 & 1688.41 & 1724.07 & 1724.07 & 1947.78
\end{array}
$$$$
\begin{array}{lllll}
1959.20 & 1959.20 & 1967.36 & 2087.60 & 2164.97
\end{array}
$$

$\begin{array}{lllll}2164.98 & 2264.40 & 2264.40 & 2353.41 & 2353.42\end{array}$

$\begin{array}{lllll}4416.18 & 4597.71 & 4605.26 & 4605.26 & 4612.86\end{array}$

$\begin{array}{llllll}4612.89 & 4635.71 & 4635.74 & 4636.21 & 4649.43\end{array}$

Zero-point correction=

.145593 (Hartree/Particle) 
XXII. Harmonic frequency analysis and zero-point energy for 1-monodeuterated methane-benzene complex with the MP2/6-311++G(d,p) method:

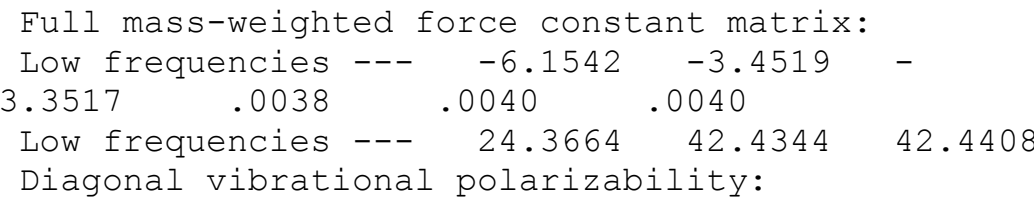

$$
8.0810770 \quad .8594665 \quad .8593569
$$

Harmonic frequencies $\left(\mathrm{cm}^{*}{ }^{*}-1\right)$, IR intensities (KM/Mole), Raman scattering activities ( $A^{* * 4 / A M U), ~ d e p o l a r i z a t i o n ~ r a t i o s ~ f o r ~ p l a n e ~ a n d ~ u n p o l a r i z e d ~}$ incident light, reduced masses (AMU), force constants (mDyne/A), and normal coordinates:

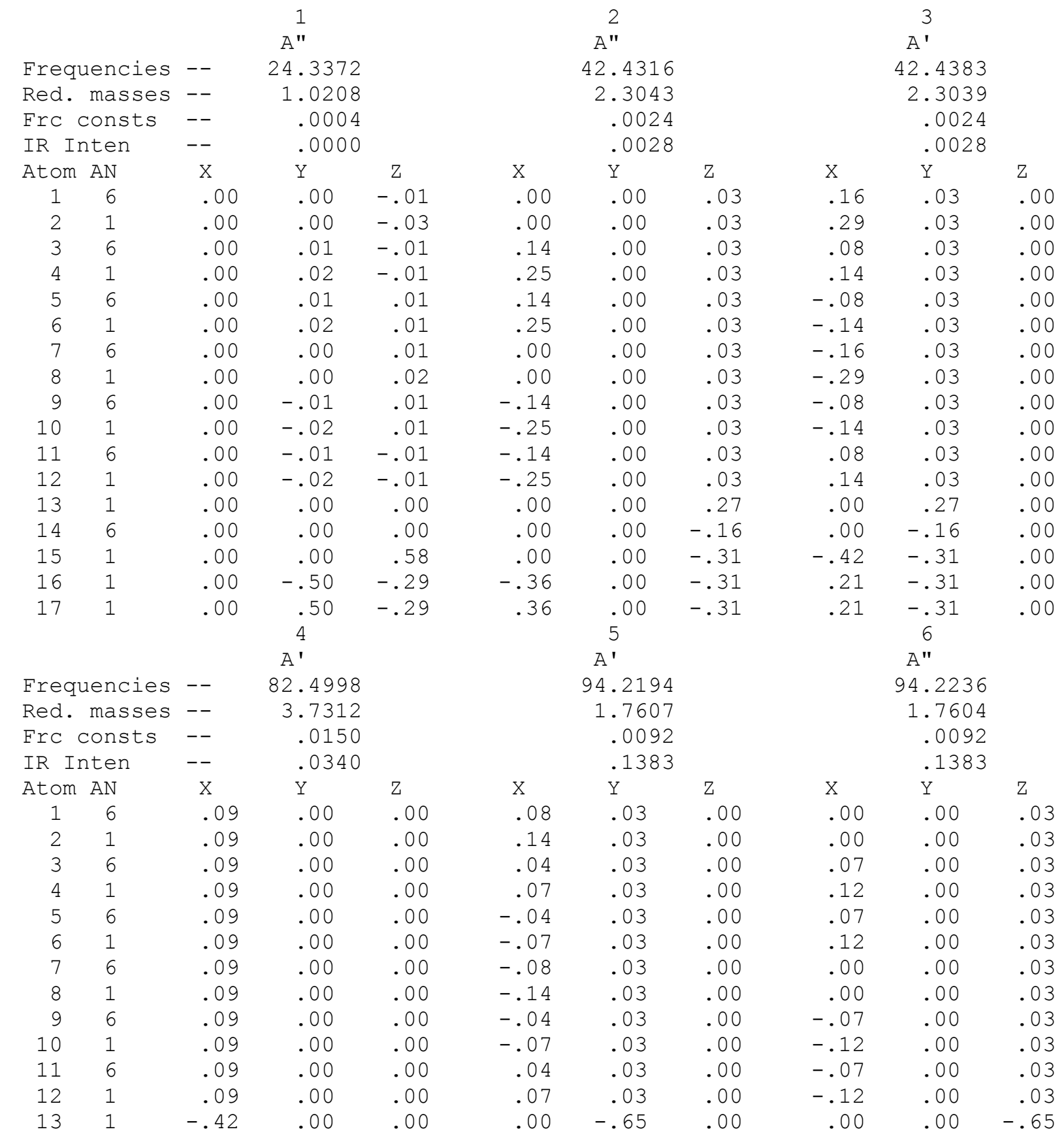




\begin{tabular}{|c|c|c|c|c|c|c|c|c|c|c|}
\hline 14 & 6 & -.42 & .00 & .00 & .00 & -.09 & .00 & .00 & .00 & -.09 \\
\hline 15 & 1 & -.42 & .00 & .00 & .55 & .11 & .00 & .00 & .00 & .11 \\
\hline 16 & 1 & -.42 & .00 & .00 & -.27 & .11 & .00 & .47 & .00 & .11 \\
\hline \multirow[t]{2}{*}{17} & 1 & -.42 & .00 & .00 & -.27 & $\dot{8}^{11}$ & .00 & -.47 & $\dot{9}^{.00}$ & .11 \\
\hline & & & $A^{\prime}$ & & & $\mathrm{A}^{\prime \prime}$ & & \multicolumn{3}{|c|}{$A^{\prime}$} \\
\hline \multicolumn{2}{|c|}{ Frequencies } & -- & \multicolumn{2}{|l|}{369.6277} & \multicolumn{3}{|c|}{369.6289} & \multicolumn{3}{|c|}{469.8032} \\
\hline Red. & masses & -- & \multicolumn{2}{|l|}{3.4123} & \multicolumn{3}{|c|}{3.4124} & & 9.9359 & \\
\hline \multicolumn{2}{|c|}{ Frc consts } & -- & \multicolumn{2}{|l|}{.2747} & \multicolumn{3}{|c|}{.2747} & \multicolumn{3}{|c|}{1.2921} \\
\hline \multicolumn{2}{|c|}{ IR Inten } & -- & .0004 & & \multicolumn{3}{|c|}{.0004} & \multicolumn{3}{|c|}{.0000} \\
\hline Atom & AN & $\mathrm{X}$ & $\mathrm{Y}$ & Z & $\mathrm{X}$ & $\mathrm{Y}$ & Z & $\mathrm{X}$ & $\mathrm{Y}$ & Z \\
\hline 1 & 6 & .27 & .00 & .00 & .00 & .00 & .00 & .37 & .00 & .00 \\
\hline 2 & 1 & .51 & .00 & .00 & .00 & .00 & .00 & .18 & .00 & .00 \\
\hline 3 & 6 & -.13 & .00 & .00 & .23 & .00 & .00 & -.37 & .00 & .00 \\
\hline 4 & 1 & -.26 & .00 & .00 & .44 & .00 & .00 & -.18 & .00 & .00 \\
\hline 5 & 6 & -.14 & .00 & .00 & -.23 & .00 & .00 & .37 & .00 & .00 \\
\hline 6 & 1 & -.25 & .00 & .00 & -.44 & .00 & .00 & .18 & .00 & .00 \\
\hline 7 & 6 & .27 & .00 & .00 & .00 & .00 & .00 & -.37 & .00 & .00 \\
\hline 8 & 1 & .51 & .00 & .00 & .00 & .00 & .00 & -.18 & .00 & .00 \\
\hline 9 & 6 & -.13 & .00 & .00 & .23 & .00 & .00 & .37 & .00 & .00 \\
\hline 10 & 1 & -.25 & .00 & .00 & .44 & .00 & .00 & .18 & .00 & .00 \\
\hline 11 & 6 & -.14 & .00 & .00 & -.23 & .00 & .00 & -.37 & .00 & .00 \\
\hline 12 & 1 & -.26 & .00 & .00 & -.44 & .00 & .00 & -.18 & .00 & .00 \\
\hline 13 & 1 & .00 & .00 & .00 & .00 & .00 & .00 & .00 & .00 & .00 \\
\hline 14 & 6 & .00 & .00 & .00 & .00 & .00 & .00 & .00 & .00 & .00 \\
\hline 15 & 1 & .00 & .00 & .00 & .00 & .00 & .00 & .00 & .00 & .00 \\
\hline 16 & 1 & .00 & .00 & .00 & .00 & .00 & .00 & .00 & .00 & .00 \\
\hline \multirow[t]{3}{*}{17} & 1 & .00 & .00 & .00 & .00 & .00 & .00 & .00 & .00 & .00 \\
\hline & & & 10 & & & 11 & & & 12 & \\
\hline & & & ?A & & & ?A & & & $A^{\prime}$ & \\
\hline Frequ & dencies & -- & 608.7809 & & & 608.7815 & & & 666.6114 & \\
\hline Red. & masses & -- & 6.0129 & & & 6.0129 & & & 1.0850 & \\
\hline Frc $C$ & yonsts & -- & 1.3130 & & & 1.3130 & & & .2841 & \\
\hline IR In & iten & -- & .0000 & & & .0000 & & & 127.8333 & \\
\hline Atom & AN & $\mathrm{X}$ & Y & Z & $\mathrm{X}$ & $\mathrm{Y}$ & Z & $\mathrm{X}$ & $\mathrm{Y}$ & Z \\
\hline 1 & 6 & .00 & .00 & .15 & .00 & .36 & .00 & -.03 & .00 & .00 \\
\hline 2 & 1 & .00 & .00 & -.23 & -.01 & .36 & .00 & .41 & .00 & .00 \\
\hline 3 & 6 & .00 & .22 & .23 & .00 & .03 & -.22 & -.03 & .00 & .00 \\
\hline 4 & 1 & -.01 & .05 & .33 & .00 & -.26 & -.05 & .40 & .00 & .00 \\
\hline 5 & 6 & .00 & .22 & -.23 & .00 & -.03 & -.22 & -.03 & .00 & .00 \\
\hline 6 & 1 & .01 & .05 & -.33 & .00 & .26 & -.05 & .41 & .00 & .00 \\
\hline 7 & 6 & .00 & .00 & -.15 & .00 & -.36 & .00 & -.03 & .00 & .00 \\
\hline 8 & 1 & .00 & .00 & .23 & -.01 & -.36 & .00 & .40 & .00 & .00 \\
\hline 9 & 6 & .00 & -.22 & -.23 & .00 & -.03 & .22 & -.03 & .00 & .00 \\
\hline 10 & 1 & -.01 & -.05 & -.33 & .00 & .26 & .05 & .41 & .00 & .00 \\
\hline 11 & 6 & .00 & -.22 & .23 & .00 & .03 & .22 & -.03 & .00 & .00 \\
\hline 12 & 1 & .01 & -.05 & .33 & .00 & -.26 & .05 & .40 & .00 & .00 \\
\hline 13 & 1 & .00 & .00 & .00 & .00 & .00 & .00 & .00 & .00 & .00 \\
\hline 14 & 6 & .00 & .00 & .00 & .00 & .00 & .00 & .00 & .00 & .00 \\
\hline 15 & 1 & .00 & .00 & .00 & .00 & .00 & .00 & .00 & .00 & .00 \\
\hline 16 & 1 & .00 & .00 & .00 & .00 & .00 & .00 & .00 & .00 & .00 \\
\hline 17 & 1 & .00 & .00 & .00 & .00 & .00 & .00 & .00 & .00 & .00 \\
\hline & & & 13 & & & 14 & & & 15 & \\
\hline & & & $A^{\prime}$ & & & $A^{\prime \prime}$ & & & $A^{\prime}$ & \\
\hline Frequ & dencies & -- & 834.3381 & & & 834.3470 & & & 897.4799 & \\
\hline Red. & masses & -- & 1.2494 & & & 1.2494 & & & 1.2780 & \\
\hline Frc $C$ & yonsts & -- & .5124 & & & .5124 & & & .6065 & \\
\hline
\end{tabular}




\begin{tabular}{|c|c|c|c|c|c|c|c|c|c|c|}
\hline IR I & nten & -- & .132 & & & .132 & & & .0013 & \\
\hline Atom & AN & $\mathrm{x}$ & $\mathrm{Y}$ & Z & $\mathrm{X}$ & $\mathrm{Y}$ & Z & $\mathrm{x}$ & $\mathrm{Y}$ & Z \\
\hline 1 & 6 & -.09 & .00 & .00 & .00 & .00 & .00 & -.09 & .00 & .00 \\
\hline 2 & 1 & .60 & .00 & .00 & .00 & .00 & .00 & .54 & .00 & .00 \\
\hline 3 & 6 & -.04 & .00 & .00 & .07 & .00 & .00 & .05 & .00 & .00 \\
\hline 4 & 1 & .27 & .00 & .00 & -.47 & .00 & .00 & -.30 & .00 & .00 \\
\hline 5 & 6 & .04 & .00 & .00 & .08 & .00 & .00 & .04 & .00 & .00 \\
\hline 6 & 1 & -.30 & .00 & .00 & -.52 & .00 & .00 & -.27 & .00 & .00 \\
\hline 7 & 6 & .08 & .00 & .00 & .00 & .00 & .00 & -.09 & .00 & .00 \\
\hline 8 & 1 & -.54 & .00 & .00 & .00 & .00 & .00 & .60 & .00 & .00 \\
\hline 9 & 6 & .04 & .00 & .00 & -.08 & .00 & .00 & .04 & .00 & .00 \\
\hline 10 & 1 & -.30 & .00 & .00 & .52 & .00 & .00 & -.27 & .00 & .00 \\
\hline 11 & 6 & -.04 & .00 & .00 & -.07 & .00 & .00 & .05 & .00 & .00 \\
\hline 12 & 1 & .27 & .00 & .00 & .47 & .00 & .00 & -.30 & .00 & .00 \\
\hline 13 & 1 & .00 & .00 & .00 & .00 & .00 & .00 & .00 & .00 & .00 \\
\hline 14 & 6 & .00 & .00 & .00 & .00 & .00 & .00 & .00 & .00 & .00 \\
\hline 15 & 1 & .00 & .00 & .00 & .00 & .00 & .00 & .00 & .00 & .00 \\
\hline 16 & 1 & .00 & .00 & .00 & .00 & .00 & .00 & .00 & .00 & .00 \\
\hline 17 & 1 & .00 & .00 & .00 & .00 & .00 & .00 & .00 & .00 & .00 \\
\hline & & & 16 & & & 17 & & & 18 & \\
\hline & & & A" & & & $A^{\prime}$ & & & $A^{\prime}$ & \\
\hline Freq & uencies & -- & 897.488 & & & .7 .656 & & & 008.5331 & \\
\hline Red. & masses & -- & 1.278 & & & 1.025 & & & 5.9699 & \\
\hline Frc & consts & -- & .606 & & & .520 & & & 3.5776 & \\
\hline IR I & nten & -- & .001 & & & .003 & & & .0334 & \\
\hline Atom & AN & $\mathrm{x}$ & $\mathrm{Y}$ & Z & $\mathrm{x}$ & $\mathrm{Y}$ & Z & $\mathrm{x}$ & $\mathrm{Y}$ & Z \\
\hline 1 & 6 & .00 & .00 & .00 & .02 & .00 & .00 & .00 & .27 & .00 \\
\hline 2 & 1 & .00 & .00 & .00 & -.41 & .00 & .00 & .00 & .30 & .00 \\
\hline 3 & 6 & -.08 & .00 & .00 & -.02 & .00 & .00 & .00 & .14 & .24 \\
\hline 4 & 1 & .52 & .00 & .00 & .41 & .00 & .00 & .00 & .15 & .26 \\
\hline 5 & 6 & .07 & .00 & .00 & .02 & .00 & .00 & .00 & -.14 & .24 \\
\hline 6 & 1 & -.47 & .00 & .00 & -.41 & .00 & .00 & .00 & -.15 & .26 \\
\hline 7 & 6 & .00 & .00 & .00 & -.02 & .00 & .00 & .00 & -.28 & .00 \\
\hline 8 & 1 & .00 & .00 & .00 & .41 & .00 & .00 & .00 & -.30 & .00 \\
\hline 9 & 6 & -.07 & .00 & .00 & .02 & .00 & .00 & .00 & -.14 & -.24 \\
\hline 10 & 1 & .47 & .00 & .00 & -.41 & .00 & .00 & .00 & -.15 & -.26 \\
\hline 11 & 6 & .08 & .00 & .00 & -.02 & .00 & .00 & .00 & .14 & -.24 \\
\hline 12 & 1 & -.52 & .00 & .00 & .41 & .00 & .00 & .00 & .15 & -.26 \\
\hline 13 & 1 & .00 & .00 & .00 & .00 & .00 & .00 & .00 & .00 & .00 \\
\hline 14 & 6 & .00 & .00 & .00 & .00 & .00 & .00 & .00 & .00 & .00 \\
\hline 15 & 1 & .00 & .00 & .00 & .00 & .00 & .00 & .00 & .00 & .00 \\
\hline 16 & 1 & .00 & .00 & .00 & .00 & .00 & .00 & .00 & .00 & .00 \\
\hline 17 & 1 & .00 & .00 & .00 & .00 & .00 & .00 & .00 & .00 & .00 \\
\hline & & & 19 & & & 20 & & & 21 & \\
\hline & & & $A^{\prime}$ & & & A" & & & $A^{\prime}$ & \\
\hline Freq & uencies & -- & 1011.172 & & & 9.436 & & & 059.4415 & \\
\hline Red. & masses & -- & 6.536 & & & 1.698 & & & 1.6985 & \\
\hline Frc & consts & -- & 3.937 & & & 1.123 & & & 1.1232 & \\
\hline IR I & nten & -- & .000 & & & 4.503 & & & 4.5033 & \\
\hline Atom & AN & $\mathrm{x}$ & Y & Z & $\mathrm{X}$ & Y & Z & $\mathrm{X}$ & Y & Z \\
\hline 1 & 6 & .00 & .29 & .00 & .00 & .00 & .08 & .00 & -.12 & .00 \\
\hline 2 & 1 & .00 & .29 & .00 & .00 & .00 & .54 & .00 & -.13 & .00 \\
\hline 3 & 6 & .00 & -.14 & -.25 & .00 & -.09 & -.07 & .00 & .03 & -.09 \\
\hline 4 & 1 & .00 & -.14 & -.25 & .00 & -.29 & .04 & .00 & .38 & -.29 \\
\hline 5 & 6 & .00 & -.15 & .25 & .00 & .09 & -.07 & .00 & .03 & .09 \\
\hline 6 & 1 & .00 & -.15 & .25 & .00 & .29 & .04 & .00 & .38 & .29 \\
\hline 7 & 6 & .00 & .29 & .00 & .00 & .00 & .08 & .00 & -.12 & .00 \\
\hline
\end{tabular}




\begin{tabular}{|c|c|c|c|c|c|c|c|c|c|c|}
\hline 8 & 1 & .00 & .29 & .00 & .00 & .00 & .54 & .00 & -.13 & .00 \\
\hline 9 & 6 & .00 & -.15 & -.25 & .00 & -.09 & -.07 & .00 & .03 & -.09 \\
\hline 10 & 1 & .00 & -.15 & -.25 & .00 & -.29 & .04 & .00 & .38 & -.29 \\
\hline 11 & 6 & .00 & -.14 & .25 & .00 & .09 & -.07 & .00 & .03 & .09 \\
\hline 12 & 1 & .00 & -.14 & .25 & .00 & .29 & .04 & .00 & .38 & .29 \\
\hline 13 & 1 & .00 & .00 & .00 & .00 & .00 & -.01 & .00 & -.01 & .00 \\
\hline 14 & 6 & .00 & .00 & .00 & .00 & .00 & .00 & .00 & .00 & .00 \\
\hline 15 & 1 & .00 & .00 & .00 & .00 & .00 & .00 & -.01 & .00 & .00 \\
\hline 16 & 1 & .00 & .00 & .00 & -.01 & .00 & .00 & .00 & .00 & .00 \\
\hline \multirow[t]{3}{*}{17} & 1 & .00 & .00 & .00 & .01 & .00 & .00 & .00 & .00 & .00 \\
\hline & & & 22 & & & 23 & & & 24 & \\
\hline & & & $\mathrm{A}^{\prime \prime}$ & & & A" & & \multirow{2}{*}{\multicolumn{3}{|c|}{1198.0247}} \\
\hline \multicolumn{2}{|c|}{ Frequencies } & -- & \multicolumn{2}{|c|}{1173.5073} & \multicolumn{3}{|c|}{1198.0234} & & & \\
\hline \multirow{2}{*}{\multicolumn{2}{|c|}{$\begin{array}{l}\text { Red. masses } \\
\text { Frc consts }\end{array}$}} & -- & 1.0313 & & \multicolumn{3}{|c|}{1.3268} & \multicolumn{3}{|c|}{1.3283} \\
\hline & & -- & .8368 & & \multicolumn{3}{|c|}{1.1220} & \\
\hline & -- & .0000 & & \multicolumn{3}{|c|}{.9894} & \multicolumn{3}{|c|}{.9956} \\
\hline \multicolumn{2}{|c|}{$\begin{array}{l}\text { IR Inten } \\
\text { Atom AN }\end{array}$} & $\mathrm{x}$ & $\mathrm{Y}$ & Z & $\mathrm{x}$ & Y & Z & $\mathrm{x}$ & $\mathrm{Y}$ & Z \\
\hline 1 & 6 & .00 & .00 & .02 & .00 & .00 & -.04 & .00 & .00 & .00 \\
\hline 2 & 1 & .00 & .00 & .41 & .00 & .00 & -.40 & .00 & .00 & .00 \\
\hline 3 & 6 & .00 & .02 & -.01 & .00 & -.02 & .01 & .00 & .03 & -.02 \\
\hline 4 & 1 & .00 & .35 & -.20 & .00 & -.18 & .10 & .00 & .30 & -.18 \\
\hline 5 & 6 & .00 & -.02 & -.01 & .00 & -.02 & -.01 & .00 & -.03 & -.0 \\
\hline 6 & 1 & .00 & -.35 & -.20 & .00 & -.17 & -.10 & .00 & -.30 & -.17 \\
\hline 7 & 6 & .00 & .00 & .02 & .00 & .00 & .04 & .00 & .00 & .00 \\
\hline 8 & 1 & .00 & .00 & .41 & .00 & .00 & .41 & .00 & .00 & .00 \\
\hline 9 & 6 & .00 & .02 & -.01 & .00 & .02 & -.01 & .00 & -.03 & .02 \\
\hline 10 & 1 & .00 & .35 & -.20 & .00 & .17 & -.10 & .00 & -.30 & .17 \\
\hline 11 & 6 & .00 & -.02 & -.01 & .00 & .02 & .01 & .00 & .03 & .02 \\
\hline 12 & 1 & .00 & -.35 & -.20 & .00 & .18 & .10 & .00 & .30 & .18 \\
\hline 13 & 1 & .00 & .00 & .00 & .00 & .00 & .37 & .00 & .37 & .00 \\
\hline 14 & 6 & .00 & .00 & .00 & .00 & .00 & -.11 & .00 & -.11 & .00 \\
\hline 15 & 1 & .00 & .00 & .00 & .00 & .00 & .29 & .39 & .05 & .00 \\
\hline 16 & 1 & .00 & .00 & .00 & .34 & .10 & .11 & -.20 & .23 & .10 \\
\hline \multirow[t]{3}{*}{17} & 1 & .00 & .00 & .00 & -.34 & -.10 & .11 & -.20 & .23 & -.1 \\
\hline & & & 25 & & & 26 & & & 27 & \\
\hline & & & A" & & & $A^{\prime}$ & & & $A^{\prime}$ & \\
\hline Frequ & encies & -- & 1198.4832 & & & 98.485 & & & 347.5856 & \\
\hline Red. & masses & -- & 1.2647 & & & 1.263 & & & 1.1642 & \\
\hline Frc & onsts & -- & 1.0703 & & & 1.069 & & & 1.2457 & \\
\hline IR In & ten & -- & .7167 & & & .710 & & & 11.9421 & \\
\hline Atom & AN & $\mathrm{x}$ & $\mathrm{Y}$ & Z & $\mathrm{x}$ & $\mathrm{Y}$ & Z & $\mathrm{x}$ & $\mathrm{Y}$ & Z \\
\hline 1 & 6 & .00 & .00 & .05 & .00 & .00 & .00 & .00 & .00 & .00 \\
\hline 2 & 1 & .00 & .00 & .47 & .00 & .00 & .00 & .00 & .00 & .00 \\
\hline 3 & 6 & .00 & .02 & -.01 & .00 & -.04 & .02 & .00 & .00 & .0 \\
\hline 4 & 1 & .00 & .20 & -.11 & .00 & -.34 & .20 & .00 & .00 & .0 \\
\hline 5 & 6 & .00 & .02 & .01 & .00 & .04 & .02 & .00 & .00 & .0 \\
\hline 6 & 1 & .00 & .20 & .11 & .00 & .35 & .21 & .00 & .00 & .0 \\
\hline 7 & 6 & .00 & .00 & -.05 & .00 & .00 & .00 & .00 & .00 & .00 \\
\hline 8 & 1 & .00 & .00 & -.46 & .00 & .00 & .00 & .00 & .00 & .0 \\
\hline 9 & 6 & .00 & -.02 & .01 & .00 & .04 & -.02 & .00 & .00 & .0 \\
\hline 10 & 1 & .00 & -.20 & .11 & .00 & .35 & -.21 & .00 & .00 & .0 \\
\hline 11 & 6 & .00 & -.02 & -.01 & .00 & -.04 & -.02 & .00 & .00 & .0 \\
\hline 12 & 1 & .00 & -.20 & -.11 & .00 & -.34 & -.20 & .00 & .00 & .0 \\
\hline 13 & 1 & .00 & .00 & .31 & .00 & .31 & .00 & .11 & .00 & .00 \\
\hline 14 & 6 & .00 & .00 & -.09 & .00 & -.09 & .00 & .11 & .00 & .0 \\
\hline 15 & 1 & .00 & .00 & .24 & .32 & .04 & .00 & -.53 & -.22 & .0 \\
\hline 16 & 1 & .28 & .08 & .09 & -.16 & .19 & .08 & -.53 & .11 & -.1 \\
\hline
\end{tabular}




\begin{tabular}{|c|c|c|c|c|c|c|c|c|c|c|}
\hline 17 & 1 & -.28 & $\begin{array}{l}-.09 \\
28 \\
\text { A" }\end{array}$ & .09 & -.16 & $\begin{array}{l}29^{19} \\
\text { A" }\end{array}$ & -.08 & -.53 & $\begin{array}{l}.11 \\
30^{\prime \prime}\end{array}$ & .19 \\
\hline \multicolumn{2}{|c|}{ Frequencies } & -- & \multicolumn{2}{|l|}{1367.3845} & \multicolumn{3}{|c|}{1450.9534} & \\
\hline \multicolumn{2}{|c|}{ Red. masses } & -- & \multicolumn{2}{|l|}{1.2471} & \multicolumn{3}{|c|}{9.4417} & \multicolumn{3}{|c|}{$\begin{array}{r}1504.6698 \\
1.9863\end{array}$} \\
\hline \multicolumn{2}{|c|}{ Frc consts } & -- & \multicolumn{2}{|l|}{1.3738} & \multicolumn{3}{|c|}{11.7114} & \multicolumn{3}{|c|}{2.6496} \\
\hline \multicolumn{2}{|c|}{ IR Inten } & -- & \multicolumn{2}{|l|}{.0000} & \multicolumn{3}{|c|}{.0000} & & 5.8039 & \\
\hline Atom & AN & $\mathrm{x}$ & $\mathrm{Y}$ & Z & $\mathrm{x}$ & $\mathrm{Y}$ & $\mathrm{Z}$ & $\mathrm{x}$ & $\mathrm{Y}$ & Z \\
\hline 1 & 6 & .00 & .00 & -.06 & .00 & .00 & .36 & .00 & .00 & .15 \\
\hline 2 & 1 & .00 & .00 & .40 & .00 & .00 & -.20 & .00 & .00 & -.53 \\
\hline 3 & 6 & .00 & .05 & -.03 & .00 & .31 & -.18 & .00 & -.10 & -.03 \\
\hline 4 & 1 & .00 & -.35 & .20 & .00 & -.17 & .10 & .00 & .18 & -.22 \\
\hline 5 & 6 & .00 & .05 & .03 & .00 & -.31 & -.18 & .00 & .10 & -.03 \\
\hline 6 & 1 & .00 & -.35 & -.20 & .00 & .17 & .10 & .00 & -.18 & -.22 \\
\hline 7 & 6 & .00 & .00 & .06 & .00 & .00 & .36 & .00 & .00 & .15 \\
\hline 8 & 1 & .00 & .00 & -.40 & .00 & .00 & -.20 & .00 & .00 & -.53 \\
\hline 9 & 6 & .00 & -.05 & .03 & .00 & .31 & -.18 & .00 & -.10 & -.03 \\
\hline 10 & 1 & .00 & .35 & -.20 & .00 & -.17 & .10 & .00 & .18 & -.22 \\
\hline 11 & 6 & .00 & -.05 & -.03 & .00 & -.31 & -.18 & .00 & .10 & -.03 \\
\hline 12 & 1 & .00 & .35 & .20 & .00 & .17 & .10 & .00 & -.18 & -.22 \\
\hline 13 & 1 & .00 & .00 & .00 & .00 & .00 & .00 & .00 & .00 & -.02 \\
\hline 14 & 6 & .00 & .00 & .00 & .00 & .00 & .00 & .00 & .00 & -.01 \\
\hline 15 & 1 & .00 & .00 & .00 & .00 & .00 & .00 & .00 & .00 & .12 \\
\hline 16 & 1 & .00 & .00 & .00 & .00 & .00 & .00 & -.05 & .06 & .01 \\
\hline 17 & 1 & .00 & .00 & .00 & .00 & .00 & .00 & .05 & -.06 & .01 \\
\hline & & & 31 & & & 32 & & & 33 & \\
\hline & & & $A^{\prime}$ & & & A" & & & $A^{\prime}$ & \\
\hline Frequ & dencies & -- & 1504.6742 & & & 11.890 & & & 511.8910 & \\
\hline Red. & masses & -- & 1.9863 & & & 1.055 & & & 1.0559 & \\
\hline Frc & consts & -- & 2.6496 & & & 1.422 & & & 1.4221 & \\
\hline IR In & nten & -- & 5.8030 & & & 5.983 & & & 5.9848 & \\
\hline Atom & AN & $\mathrm{x}$ & $\mathrm{Y}$ & Z & $\mathrm{X}$ & $\mathrm{Y}$ & Z & $\mathrm{x}$ & Y & Z \\
\hline 1 & 6 & .00 & .09 & .00 & .00 & .00 & -.01 & .00 & -.01 & .00 \\
\hline 2 & 1 & .00 & .11 & .00 & .00 & .00 & .05 & .00 & -.01 & .00 \\
\hline 3 & 6 & .00 & -.09 & .10 & .00 & .01 & .00 & .00 & .01 & -.01 \\
\hline 4 & 1 & .00 & .43 & -.18 & .00 & -.02 & .02 & .00 & -.04 & .02 \\
\hline 5 & 6 & .00 & -.09 & -.10 & .00 & -.01 & .00 & .00 & .01 & .01 \\
\hline 6 & 1 & .00 & .43 & .18 & .00 & .02 & .02 & .00 & -.04 & -.02 \\
\hline 7 & 6 & .00 & .09 & .00 & .00 & .00 & -.01 & .00 & -.01 & .00 \\
\hline 8 & 1 & .00 & .11 & .00 & .00 & .00 & .05 & .00 & -.01 & .00 \\
\hline 9 & 6 & .00 & -.09 & .10 & .00 & .01 & .00 & .00 & .01 & -.01 \\
\hline 10 & 1 & .00 & .43 & -.18 & .00 & -.02 & .02 & .00 & -.04 & .02 \\
\hline 11 & 6 & .00 & -.09 & -.10 & .00 & -.01 & .00 & .00 & .01 & .01 \\
\hline 12 & 1 & .00 & .43 & .18 & .00 & .02 & .02 & .00 & -.04 & -.02 \\
\hline 13 & 1 & .00 & .02 & .00 & .00 & .00 & -.15 & .00 & .15 & .00 \\
\hline 14 & 6 & .00 & .01 & .00 & .00 & .00 & -.04 & .00 & .04 & .00 \\
\hline 15 & 1 & .06 & .03 & .00 & .00 & .00 & .68 & .39 & .17 & .00 \\
\hline 16 & 1 & -.03 & -.08 & -.06 & -.34 & .37 & .05 & -.19 & -.47 & -.37 \\
\hline 17 & 1 & -.03 & -.08 & .06 & .34 & -.37 & .05 & -.19 & -.47 & .37 \\
\hline & & & 34 & & & 35 & & & 36 & \\
\hline & & & $A^{\prime}$ & & & $\mathrm{A}^{\prime \prime}$ & & & $A^{\prime}$ & \\
\hline Frequ & dencies & -- & 1635.7060 & & & 35.707 & & & 2332.4337 & \\
\hline Red. & masses & -- & 5.5549 & & & 5.555 & & & 2.2107 & \\
\hline Frc & consts & -- & 8.7566 & & & 8.756 & & & 7.0859 & \\
\hline IR Ir & nten & -- & .0000 & & & .000 & & & .1704 & \\
\hline Atom & AN & $\mathrm{X}$ & $\mathrm{Y}$ & Z & $\mathrm{x}$ & Y & Z & $\mathrm{x}$ & $\mathrm{Y}$ & Z \\
\hline 1 & 6 & .00 & .15 & .00 & .00 & .00 & .34 & .00 & .00 & .00 \\
\hline
\end{tabular}




\begin{tabular}{|c|c|c|c|c|c|c|c|c|c|c|}
\hline 2 & 1 & .00 & .17 & .00 & .00 & .00 & -.41 & .00 & .00 & .00 \\
\hline 3 & 6 & .00 & -.29 & .08 & .00 & .08 & -.20 & .00 & .00 & .00 \\
\hline 4 & 1 & .00 & .26 & -.25 & .00 & -.25 & -.03 & .00 & .00 & .00 \\
\hline 5 & 6 & .00 & .29 & .08 & .00 & .08 & .20 & .00 & .00 & .00 \\
\hline 6 & 1 & .00 & -.26 & -.25 & .00 & -.25 & .03 & .00 & .00 & .00 \\
\hline 7 & 6 & .00 & -.15 & .00 & .00 & .00 & -.34 & .00 & .00 & .00 \\
\hline 8 & 1 & .00 & -.17 & .00 & .00 & .00 & .41 & .00 & .00 & .00 \\
\hline 9 & 6 & .00 & .29 & -.08 & .00 & -.08 & .20 & .00 & .00 & .00 \\
\hline 10 & 1 & .00 & -.26 & .25 & .00 & .25 & .03 & .00 & .00 & .00 \\
\hline 11 & 6 & .00 & -.29 & -.08 & .00 & -.08 & -.20 & .00 & .00 & .0 \\
\hline 12 & 1 & .00 & .26 & .25 & .00 & .25 & -.03 & .00 & .00 & .0 \\
\hline 13 & 1 & .00 & .00 & .00 & .00 & .00 & .00 & .97 & .00 & .0 \\
\hline 14 & 6 & .00 & .00 & .00 & .00 & .00 & .00 & -.15 & .00 & .00 \\
\hline 15 & 1 & .00 & .00 & .00 & .00 & .00 & .00 & -.04 & .10 & .00 \\
\hline 16 & 1 & .00 & .00 & .00 & .00 & .00 & .00 & -.04 & -.05 & .0 \\
\hline \multirow[t]{3}{*}{17} & 1 & .00 & .00 & .00 & .00 & .00 & .00 & -.04 & -.05 & -.0 \\
\hline & & & 37 & & & 38 & & & 39 & \\
\hline & & & $A^{\prime}$ & & & $A^{\prime}$ & & & A" & \\
\hline \multicolumn{2}{|c|}{ Frequencies } & -- & 3105.0381 & & \multicolumn{3}{|c|}{3195.5720} & \multicolumn{3}{|c|}{3200.5536} \\
\hline \multirow{2}{*}{\multicolumn{2}{|c|}{$\begin{array}{l}\text { Red. masses } \\
\text { Frc consts }\end{array}$}} & -- & 1.0415 & & \multicolumn{3}{|c|}{1.0839} & \multicolumn{3}{|c|}{1.1016} \\
\hline & & -- & 5.9163 & & \multicolumn{3}{|c|}{6.5215} & \multicolumn{3}{|c|}{6.6488} \\
\hline & -- & 9.3241 & & \multicolumn{3}{|c|}{.0000} & \multicolumn{3}{|c|}{17.3394} \\
\hline Atom & IR Inten & $x$ & $\mathrm{Y}$ & Z & $\mathrm{x}$ & $\mathrm{Y}$ & Z & $\mathrm{x}$ & $\mathrm{Y}$ & Z \\
\hline 1 & 6 & .00 & .00 & .00 & .00 & -.03 & .00 & .00 & .00 & .00 \\
\hline 2 & 1 & .00 & .00 & .00 & .00 & .41 & .00 & .00 & .00 & .0 \\
\hline 3 & 6 & .00 & .00 & .00 & .00 & .02 & .03 & .00 & .00 & .00 \\
\hline 4 & 1 & .00 & .00 & .00 & .00 & -.20 & -.35 & .00 & .00 & .0 \\
\hline 5 & 6 & .00 & .00 & .00 & .00 & .02 & -.03 & .00 & .00 & .0 \\
\hline 6 & 1 & .00 & .00 & .00 & .00 & -.20 & .35 & .00 & -.01 & .0 \\
\hline 7 & 6 & .00 & .00 & .00 & .00 & -.03 & .00 & .00 & .00 & .0 \\
\hline 8 & 1 & .00 & .00 & .00 & .00 & .41 & .00 & .00 & .00 & .0 \\
\hline 9 & 6 & .00 & .00 & .00 & .00 & .02 & .03 & .00 & .00 & .00 \\
\hline 10 & 1 & .00 & .00 & .00 & .00 & -.20 & -.35 & .00 & .01 & .0 \\
\hline 11 & 6 & .00 & .00 & .00 & .00 & .02 & -.03 & .00 & .00 & .0 \\
\hline 12 & 1 & .00 & .00 & .00 & .00 & -.20 & .35 & .00 & .00 & .0 \\
\hline 13 & 1 & -.05 & .00 & .00 & .00 & .00 & .00 & .00 & .00 & .0 \\
\hline 14 & 6 & .05 & .00 & .00 & .00 & .00 & .00 & .00 & .00 & .0 \\
\hline 15 & 1 & -.18 & .55 & .00 & .00 & .00 & .00 & .00 & .00 & .0 \\
\hline 16 & 1 & -.18 & -.27 & .47 & .00 & .00 & .00 & .24 & .34 & -.5 \\
\hline \multirow[t]{3}{*}{17} & 1 & -.18 & -.27 & -.47 & .00 & .00 & .00 & -.24 & -.34 & -.5 \\
\hline & & & 40 & & & 41 & & & 42 & \\
\hline & & & $A^{\prime}$ & & & $\mathrm{A}^{\prime \prime}$ & & & $A^{\prime}$ & \\
\hline \multirow{2}{*}{\multicolumn{2}{|c|}{$\begin{array}{l}\text { Frequencies } \\
\text { Red. masses }\end{array}$}} & -- & 3200.5570 & & & 06.104 & & & 206.1231 & \\
\hline & & -- & 1.1016 & & & 1.088 & & & 1.0883 & \\
\hline Frc & onsts & -- & 6.6488 & & & 6.590 & & & 6.5909 & \\
\hline IR Ir & ten & -- & 17.3398 & & & .003 & & & .0032 & \\
\hline Atom & AN & $\mathrm{X}$ & $\mathrm{Y}$ & Z & $\mathrm{X}$ & $\mathrm{Y}$ & Z & $\mathrm{x}$ & $\mathrm{Y}$ & Z \\
\hline 1 & 6 & .00 & .00 & .00 & .00 & .00 & .00 & .00 & -.05 & .00 \\
\hline 2 & 1 & .00 & .01 & .00 & .00 & .00 & .00 & .00 & .58 & .0 \\
\hline 3 & 6 & .00 & .00 & .00 & .00 & .02 & .04 & .00 & .01 & .0 \\
\hline 4 & 1 & .00 & .00 & .00 & .00 & -.25 & -.43 & .00 & -.15 & -.2 \\
\hline 5 & 6 & .00 & .00 & .00 & .00 & .02 & -.04 & .00 & -.01 & .0 \\
\hline 6 & 1 & .00 & .00 & -.01 & .00 & -.25 & .43 & .00 & .15 & -.2 \\
\hline 7 & 6 & .00 & .00 & .00 & .00 & .00 & .00 & .00 & .05 & .0 \\
\hline 8 & 1 & .00 & .00 & .00 & .00 & .00 & .00 & .00 & -.57 & .0 \\
\hline 9 & 6 & .00 & .00 & .00 & .00 & -.02 & -.04 & .00 & -.01 & -.0 \\
\hline 10 & 1 & .00 & .00 & .01 & .00 & .25 & .43 & .00 & .15 & .2 \\
\hline
\end{tabular}




\begin{tabular}{|c|c|c|c|c|c|c|c|c|c|c|}
\hline 11 & 6 & .00 & .00 & .00 & .00 & -.02 & .04 & .00 & .01 & -.02 \\
\hline 12 & 1 & .00 & .00 & .00 & .00 & .25 & -.43 & .00 & -.15 & .25 \\
\hline 13 & 1 & .00 & .01 & .00 & .00 & .00 & .00 & .00 & .00 & .00 \\
\hline 14 & 6 & .00 & .09 & .00 & .00 & .00 & .00 & .00 & .00 & .00 \\
\hline 15 & 1 & .28 & -.76 & .00 & .00 & .00 & .00 & .00 & .01 & .00 \\
\hline 16 & 1 & -.14 & -.18 & .34 & .00 & .00 & .01 & .00 & .00 & \\
\hline \multirow[t]{3}{*}{17} & 1 & -.14 & -.18 & -.34 & .00 & .00 & .01 & .00 & .00 & .0 \\
\hline & & & 43 & & & 44 & & & 45 & \\
\hline & & & A" & & & $A^{\prime}$ & & & $A^{\prime}$ & \\
\hline \multicolumn{2}{|c|}{ Frequencies } & -- & 3221.9828 & & \multicolumn{3}{|c|}{3222.0017} & \multicolumn{3}{|c|}{3231.5112} \\
\hline \multicolumn{2}{|c|}{ Red. masses } & -- & 1.0964 & & \multicolumn{3}{|c|}{1.0964} & \multicolumn{3}{|c|}{1.1012} \\
\hline \multicolumn{2}{|c|}{ Frc consts } & -- & 6.7058 & & \multicolumn{3}{|c|}{6.7059} & \multicolumn{3}{|c|}{6.7755} \\
\hline \multicolumn{2}{|c|}{ IR Inten } & -- & 26.1091 & & \multicolumn{3}{|c|}{26.1080} & \multicolumn{3}{|c|}{.1071} \\
\hline Atom & AN & $\mathrm{X}$ & $\mathrm{Y}$ & $\mathrm{Z}$ & $\mathrm{X}$ & $\mathrm{Y}$ & Z & $\mathrm{x}$ & $\mathrm{Y}$ & Z \\
\hline 1 & 6 & .00 & .00 & .00 & .00 & -.05 & .00 & .00 & -.04 & ${ }^{\circ}$ \\
\hline 2 & 1 & .00 & .00 & .00 & .00 & .57 & .00 & .00 & .41 & .00 \\
\hline 3 & 6 & .00 & -.02 & -.04 & .00 & -.01 & -.02 & .00 & -.02 & -.0 \\
\hline 4 & 1 & .00 & .25 & .43 & .00 & .14 & .25 & .00 & .20 & .3 \\
\hline 5 & 6 & .00 & .02 & -.04 & .00 & -.01 & .02 & .00 & .02 & -.0 \\
\hline 6 & 1 & .00 & -.25 & .43 & .00 & .14 & -.25 & .00 & -.20 &. \\
\hline 7 & 6 & .00 & .00 & .00 & .00 & -.05 & .00 & .00 & .04 & .0 \\
\hline 8 & 1 & .00 & .00 & .00 & .00 & .58 & .00 & .00 & -.41 & .0 \\
\hline 9 & 6 & .00 & -.02 & -.04 & .00 & -.01 & -.02 & .00 & .02 & .0 \\
\hline 10 & 1 & .00 & .25 & .43 & .00 & .14 & .25 & .00 & -.20 & -.3 \\
\hline 11 & 6 & .00 & .02 & -.04 & .00 & -.01 & .02 & .00 & -.02 & .0 \\
\hline 12 & 1 & .00 & -.25 & .43 & .00 & .14 & -.25 & .00 & .20 & -.3 \\
\hline 13 & 1 & .00 & .00 & .00 & .00 & .00 & .00 & .00 & .00 & .0 \\
\hline 14 & 6 & .00 & .00 & .00 & .00 & .00 & .00 & .00 & .00 & \\
\hline 15 & 1 & .00 & .00 & .00 & .00 & .01 & .00 & .00 & .00 & .0 \\
\hline 16 & 1 & .00 & .00 & .01 & .00 & .00 & .00 & .00 & .00 & .0 \\
\hline 17 & 1 & .00 & .00 & .01 & .00 & .00 & .00 & .00 & .00 & \\
\hline
\end{tabular}

- Thermochemistry -

Temperature 298.150 Kelvin. Pressure 1.00000 Atm.

Atom 1 has atomic number 6 and mass 12.00000

Atom 2 has atomic number 1 and mass 1.00783

Atom 3 has atomic number 6 and mass 12.00000

Atom 4 has atomic number 1 and mass 1.00783

Atom 5 has atomic number 6 and mass 12.00000

Atom 6 has atomic number 1 and mass 1.00783

Atom 7 has atomic number 6 and mass 12.00000

Atom 8 has atomic number 1 and mass 1.00783

Atom 9 has atomic number 6 and mass 12.00000

Atom 10 has atomic number 1 and mass 1.00783

Atom 11 has atomic number 6 and mass 12.00000

Atom 12 has atomic number 1 and mass 1.00783

Atom 13 has atomic number 1 and mass 2.01410

Atom 14 has atomic number 6 and mass 12.00000

Atom 15 has atomic number 1 and mass 1.00783

Atom 16 has atomic number 1 and mass 1.00783

Atom 17 has atomic number 1 and mass 1.00783

Molecular mass: 95.08453 amu.

Principal axes and moments of inertia in atomic units:

$$
\begin{array}{crrr}
\text { EIGENVALUES -- } & 649.23621 & 981.95231 & 981.95302 \\
\text { X } & 1.00000 & .00000 & .00000
\end{array}
$$




$\begin{array}{lrrr}Y & .00000 & 1.00000 & .00000 \\ Z & .00000 & .00000 & 1.00000\end{array}$

This molecule is an asymmetric top.

Rotational symmetry number 1 .

Warning -- assumption of classical behavior for rotation may cause significant error

$\begin{array}{llll}\text { Rotational temperatures (Kelvin) } & .13341 & .08821 & .08821\end{array}$

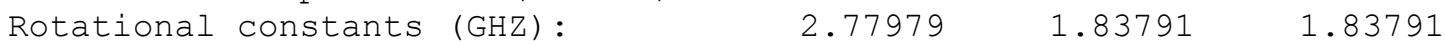

Zero-point vibrational energy $\quad 374204.7$ (Joules/Mol)

89.43707 (Kcal/Mol)

Warning -- explicit consideration of 11 degrees of freedom as vibrations may cause significant error (Kelvin)

$\begin{array}{lll}135.57 & 531.81 & 531.81\end{array}$

$675.94 \quad 875.90$

$\begin{array}{lllll}875.90 & 959.10 & 1200.42 & 1200.44 & 1291.27\end{array}$

$\begin{array}{lllll}1291.28 & 1334.69 & 1451.05 & 1454.85 & 1524.29\end{array}$

$\begin{array}{lllll}1524.30 & 1688.41 & 1723.69 & 1723.69 & 1724.35\end{array}$

$\begin{array}{lllll}1724.35 & 1938.87 & 1967.36 & 2087.60 & 2164.88\end{array}$

$\begin{array}{lllll}2164.89 & 2175.27 & 2175.27 & 2353.41 & 2353.42\end{array}$

$\begin{array}{lllll}3355.85 & 4467.45 & 4597.71 & 4604.88 & 4604.88\end{array}$

$\begin{array}{llllll}4612.86 & 4612.89 & 4635.71 & 4635.74 & 4649.42\end{array}$

Zero-point correction=

.142527 (Hartree/Particle) 
XXIII. Harmonic frequency analysis and zero-point energy for 1,2-dideuterated methane-benzene complex with the MP2/6-311++G(d,p) method:

Full mass-weighted force constant matrix:

$\begin{array}{ccccc}\text { Low frequencies } & -- & -6.1474 & -3.3843 & - \\ 3.2802 \quad .0038 & .0039 & .0040 & \\ \text { Low frequencies }--- & 21.2208 & 40.0678 & 41.8471 \\ \text { Diagonal vibrational polarizability: } & \end{array}$

Diagonal vibrational polarizability:

$$
8.0810771 \quad .8717475 \quad .8695268
$$

Harmonic frequencies ( $\left.\mathrm{cm}^{\star} \star-1\right)$, IR intensities (KM/Mole), Raman scattering activities ( $A^{* * 4 / A M U), ~ d e p o l a r i z a t i o n ~ r a t i o s ~ f o r ~ p l a n e ~ a n d ~ u n p o l a r i z e d ~}$ incident light, reduced masses (AMU), force constants (mDyne/A), and normal coordinates:

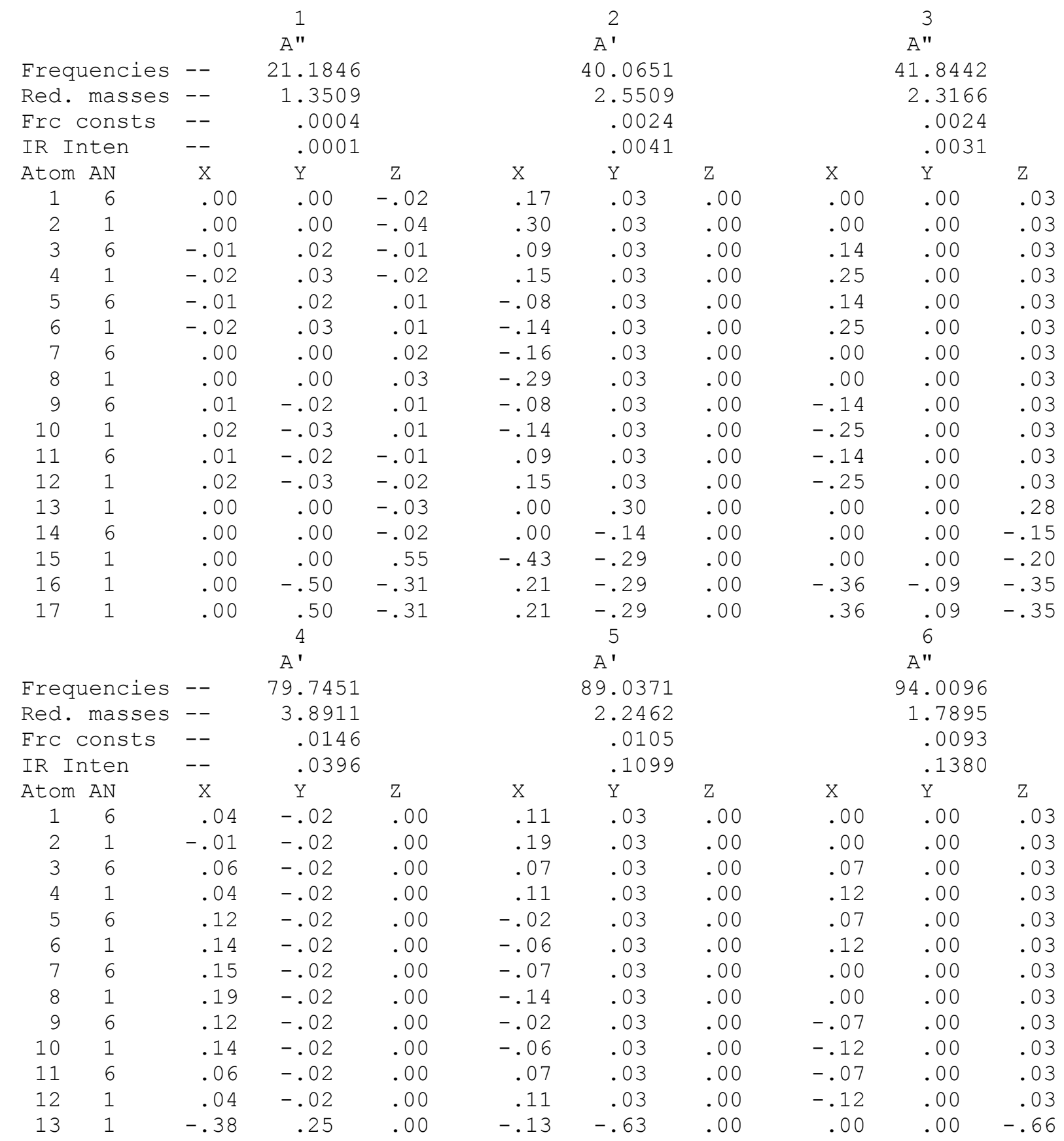




\begin{tabular}{|c|c|c|c|c|c|c|c|c|c|c|}
\hline 14 & 6 & -.39 & .07 & .00 & -.13 & -.11 & .00 & .00 & .00 & -.09 \\
\hline 15 & 1 & -.57 & .00 & .00 & .37 & .07 & .00 & .00 & .00 & .07 \\
\hline 16 & 1 & -.29 & .00 & .00 & -.38 & .07 & .00 & .47 & .02 & .11 \\
\hline \multirow[t]{2}{*}{17} & 1 & -.29 & .00 & .00 & -.38 & $\dot{8}^{.07}$ & .00 & -.47 & $\begin{array}{c}-.02 \\
9\end{array}$ & .11 \\
\hline & & \multicolumn{3}{|c|}{$A^{\prime}$} & \multicolumn{3}{|c|}{$A^{\prime \prime}$} & \multicolumn{3}{|c|}{$A^{\prime}$} \\
\hline \multicolumn{2}{|c|}{ Frequencies } & -- & 369.6277 & & \multicolumn{3}{|c|}{369.6289} & \multicolumn{3}{|c|}{469.8032} \\
\hline Red. & masses & -- & 3.4123 & & \multicolumn{3}{|c|}{3.4124} & \multicolumn{3}{|c|}{9.9359} \\
\hline \multicolumn{2}{|c|}{ Frc consts } & -- & .2747 & & \multicolumn{3}{|c|}{.2747} & \multicolumn{3}{|c|}{1.2921} \\
\hline \multicolumn{2}{|c|}{ IR Inten } & -- & .0004 & & \multicolumn{3}{|c|}{.0004} & \multicolumn{3}{|c|}{.0000} \\
\hline Atom & AN & $\mathrm{X}$ & Y & Z & $\mathrm{X}$ & $\mathrm{Y}$ & Z & $\mathrm{X}$ & Y & Z \\
\hline 1 & 6 & .27 & .00 & .00 & .00 & .00 & .00 & .37 & .00 & .00 \\
\hline 2 & 1 & .51 & .00 & .00 & .00 & .00 & .00 & .18 & .00 & .00 \\
\hline 3 & 6 & -.13 & .00 & .00 & .23 & .00 & .00 & -.37 & .00 & .00 \\
\hline 4 & 1 & -.26 & .00 & .00 & .44 & .00 & .00 & -.18 & .00 & .00 \\
\hline 5 & 6 & -.14 & .00 & .00 & -.23 & .00 & .00 & .37 & .00 & .00 \\
\hline 6 & 1 & -.25 & .00 & .00 & -.44 & .00 & .00 & .18 & .00 & .00 \\
\hline 7 & 6 & .27 & .00 & .00 & .00 & .00 & .00 & -.37 & .00 & .00 \\
\hline 8 & 1 & .51 & .00 & .00 & .00 & .00 & .00 & -.18 & .00 & .00 \\
\hline 9 & 6 & -.13 & .00 & .00 & .23 & .00 & .00 & .37 & .00 & .00 \\
\hline 10 & 1 & -.25 & .00 & .00 & .44 & .00 & .00 & .18 & .00 & .00 \\
\hline 11 & 6 & -.14 & .00 & .00 & -.23 & .00 & .00 & -.37 & .00 & .00 \\
\hline 12 & 1 & -.26 & .00 & .00 & -.44 & .00 & .00 & -.18 & .00 & .00 \\
\hline 13 & 1 & .00 & .00 & .00 & .00 & .00 & .00 & .00 & .00 & .00 \\
\hline 14 & 6 & .00 & .00 & .00 & .00 & .00 & .00 & .00 & .00 & .00 \\
\hline 15 & 1 & .00 & .00 & .00 & .00 & .00 & .00 & .00 & .00 & .00 \\
\hline 16 & 1 & .00 & .00 & .00 & .00 & .00 & .00 & .00 & .00 & .00 \\
\hline \multirow[t]{3}{*}{17} & 1 & .00 & .00 & .00 & .00 & .00 & .00 & .00 & .00 & .00 \\
\hline & & & 10 & & & 11 & & & 12 & \\
\hline & & & ?A & & & ?A & & & $A^{\prime}$ & \\
\hline Frequ & dencies & -- & 608.7809 & & & 608.7815 & & & 666.6108 & \\
\hline Red. & masses & -- & 6.0129 & & & 6.0129 & & & 1.0850 & \\
\hline Frc $C$ & yonsts & -- & 1.3130 & & & 1.3130 & & & .2841 & \\
\hline IR In & iten & -- & .0000 & & & .0000 & & & 127.8483 & \\
\hline Atom & AN & $\mathrm{X}$ & Y & Z & $\mathrm{X}$ & Y & Z & $\mathrm{X}$ & $\mathrm{Y}$ & Z \\
\hline 1 & 6 & .00 & .00 & .15 & .00 & .36 & .00 & -.03 & .00 & .00 \\
\hline 2 & 1 & .00 & .00 & -.23 & -.01 & .36 & .00 & .41 & .00 & .00 \\
\hline 3 & 6 & .00 & .22 & .23 & .00 & .03 & -.22 & -.03 & .00 & .00 \\
\hline 4 & 1 & -.01 & .05 & .33 & .00 & -.26 & -.05 & .40 & .00 & .00 \\
\hline 5 & 6 & .00 & .22 & -.23 & .00 & -.03 & -.22 & -.03 & .00 & .00 \\
\hline 6 & 1 & .01 & .05 & -.33 & .00 & .26 & -.05 & .41 & .00 & .00 \\
\hline 7 & 6 & .00 & .00 & -.15 & .00 & -.36 & .00 & -.03 & .00 & .00 \\
\hline 8 & 1 & .00 & .00 & .23 & -.01 & -.36 & .00 & .40 & .00 & .00 \\
\hline 9 & 6 & .00 & -.22 & -.23 & .00 & -.03 & .22 & -.03 & .00 & .00 \\
\hline 10 & 1 & -.01 & -.05 & -.33 & .00 & .26 & .05 & .41 & .00 & .00 \\
\hline 11 & 6 & .00 & -.22 & .23 & .00 & .03 & .22 & -.03 & .00 & .00 \\
\hline 12 & 1 & .01 & -.05 & .33 & .00 & -.26 & .05 & .40 & .00 & .00 \\
\hline 13 & 1 & .00 & .00 & .00 & .00 & .00 & .00 & .00 & .00 & .00 \\
\hline 14 & 6 & .00 & .00 & .00 & .00 & .00 & .00 & .00 & .00 & .00 \\
\hline 15 & 1 & .00 & .00 & .00 & .00 & .00 & .00 & .00 & .00 & .00 \\
\hline 16 & 1 & .00 & .00 & .00 & .00 & .00 & .00 & .00 & .00 & .00 \\
\hline 17 & 1 & .00 & .00 & .00 & .00 & .00 & .00 & .00 & .00 & .00 \\
\hline & & & 13 & & & 14 & & & 15 & \\
\hline & & & $A^{\prime}$ & & & $\mathrm{A}^{\prime \prime}$ & & & $A^{\prime}$ & \\
\hline Frequ & dencies & -- & 834.3355 & & & 834.3465 & & & 897.4795 & \\
\hline Red. & masses & -- & 1.2494 & & & 1.2494 & & & 1.2780 & \\
\hline Frc $C$ & yonsts & -- & .5124 & & & .5124 & & & .6065 & \\
\hline
\end{tabular}




\begin{tabular}{|c|c|c|c|c|c|c|c|c|c|c|}
\hline IR I & nten & -- & .1330 & & & .13 & & & .0013 & \\
\hline Atom & AN & $\mathrm{x}$ & $\mathrm{Y}$ & Z & $\mathrm{X}$ & $\mathrm{Y}$ & Z & $\mathrm{x}$ & $\mathrm{Y}$ & Z \\
\hline 1 & 6 & -.09 & .00 & .00 & .00 & .00 & .00 & -.09 & .00 & .00 \\
\hline 2 & 1 & .60 & .00 & .00 & .00 & .00 & .00 & .54 & .00 & .00 \\
\hline 3 & 6 & -.04 & .00 & .00 & .07 & .00 & .00 & .05 & .00 & .00 \\
\hline 4 & 1 & .27 & .00 & .00 & -.47 & .00 & .00 & -.30 & .00 & .00 \\
\hline 5 & 6 & .04 & .00 & .00 & .08 & .00 & .00 & .04 & .00 & .00 \\
\hline 6 & 1 & -.30 & .00 & .00 & -.52 & .00 & .00 & -.27 & .00 & .00 \\
\hline 7 & 6 & .08 & .00 & .00 & .00 & .00 & .00 & -.09 & .00 & .00 \\
\hline 8 & 1 & -.54 & .00 & .00 & .00 & .00 & .00 & .60 & .00 & .00 \\
\hline 9 & 6 & .04 & .00 & .00 & -.08 & .00 & .00 & .04 & .00 & .00 \\
\hline 10 & 1 & -.30 & .00 & .00 & .52 & .00 & .00 & -.27 & .00 & .00 \\
\hline 11 & 6 & -.04 & .00 & .00 & -.07 & .00 & .00 & .05 & .00 & .00 \\
\hline 12 & 1 & .27 & .00 & .00 & .47 & .00 & .00 & -.30 & .00 & .00 \\
\hline 13 & 1 & .00 & .01 & .00 & .00 & .00 & .00 & .00 & .00 & .00 \\
\hline 14 & 6 & .00 & .00 & .00 & .00 & .00 & .00 & .00 & .00 & .00 \\
\hline 15 & 1 & .00 & .00 & .00 & .00 & .00 & .00 & .00 & .00 & .00 \\
\hline 16 & 1 & .00 & .00 & .00 & .00 & .00 & .00 & .00 & .00 & .00 \\
\hline 17 & 1 & .00 & .00 & .00 & .00 & .00 & .00 & .00 & .00 & .00 \\
\hline & & & 16 & & & 17 & & & 18 & \\
\hline & & & A" & & & $A^{\prime}$ & & & $A^{\prime}$ & \\
\hline Freq & uencies & -- & 897.487 & & & .7 .65 & & & 008.5302 & \\
\hline Red. & masses & -- & 1.278 & & & 1.02 & & & 5.9693 & \\
\hline Frc & consts & -- & .606 & & & .52 & & & 3.5773 & \\
\hline IR I & nten & -- & .001 & & & .00 & & & .0303 & \\
\hline Atom & AN & $\mathrm{x}$ & $\mathrm{Y}$ & Z & $\mathrm{x}$ & $\mathrm{Y}$ & Z & $\mathrm{x}$ & $\mathrm{Y}$ & Z \\
\hline 1 & 6 & .00 & .00 & .00 & .02 & .00 & .00 & .00 & .27 & .00 \\
\hline 2 & 1 & .00 & .00 & .00 & -.41 & .00 & .00 & .00 & .30 & .00 \\
\hline 3 & 6 & -.08 & .00 & .00 & -.02 & .00 & .00 & .00 & .14 & .24 \\
\hline 4 & 1 & .52 & .00 & .00 & .41 & .00 & .00 & .00 & .15 & .26 \\
\hline 5 & 6 & .07 & .00 & .00 & .02 & .00 & .00 & .00 & -.14 & .24 \\
\hline 6 & 1 & -.47 & .00 & .00 & -.41 & .00 & .00 & .00 & -.15 & .26 \\
\hline 7 & 6 & .00 & .00 & .00 & -.02 & .00 & .00 & .00 & -.28 & .00 \\
\hline 8 & 1 & .00 & .00 & .00 & .41 & .00 & .00 & .00 & -.30 & .00 \\
\hline 9 & 6 & -.07 & .00 & .00 & .02 & .00 & .00 & .00 & -.14 & -.24 \\
\hline 10 & 1 & .47 & .00 & .00 & -.41 & .00 & .00 & .00 & -.15 & -.26 \\
\hline 11 & 6 & .08 & .00 & .00 & -.02 & .00 & .00 & .00 & .14 & -.24 \\
\hline 12 & 1 & -.52 & .00 & .00 & .41 & .00 & .00 & .00 & .15 & -.26 \\
\hline 13 & 1 & .00 & .00 & .00 & .00 & .00 & .00 & .00 & -.01 & .00 \\
\hline 14 & 6 & .00 & .00 & .00 & .00 & .00 & .00 & .00 & .00 & .00 \\
\hline 15 & 1 & .00 & .00 & .00 & .00 & .00 & .00 & -.01 & .00 & .00 \\
\hline 16 & 1 & .00 & .00 & .00 & .00 & .00 & .00 & .00 & .00 & .00 \\
\hline 17 & 1 & .00 & .00 & .00 & .00 & .00 & .00 & .00 & .00 & .00 \\
\hline & & & 19 & & & 20 & & & 21 & \\
\hline & & & $A^{\prime}$ & & & $A^{\prime}$ & & & A" & \\
\hline Freq & uencies & -- & 1011.172 & & & 8.80 & & & 059.4285 & \\
\hline Red. & masses & -- & 6.536 & & & 1.77 & & & 1.6986 & \\
\hline Frc & consts & -- & 3.937 & & & 1.17 & & & 1.1233 & \\
\hline IR I & nten & -- & .000 & & & 3.25 & & & 4.4409 & \\
\hline Atom & AN & $\mathrm{x}$ & Y & Z & $\mathrm{X}$ & Y & Z & $\mathrm{X}$ & Y & Z \\
\hline 1 & 6 & .00 & .29 & .00 & .00 & -.11 & .00 & .00 & .00 & .08 \\
\hline 2 & 1 & .00 & .29 & .00 & .00 & -.12 & .00 & .00 & .00 & .54 \\
\hline 3 & 6 & .00 & -.14 & -.25 & .00 & .03 & -.08 & .00 & -.09 & -.07 \\
\hline 4 & 1 & .00 & -.14 & -.25 & .00 & .35 & -.27 & .00 & -.29 & .04 \\
\hline 5 & 6 & .00 & -.15 & .25 & .00 & .03 & .08 & .00 & .09 & -.07 \\
\hline 6 & 1 & .00 & -.15 & .25 & .00 & .35 & .27 & .00 & .29 & .04 \\
\hline 7 & 6 & .00 & .29 & .00 & .00 & -.11 & .00 & .00 & .00 & .08 \\
\hline
\end{tabular}




\begin{tabular}{|c|c|c|c|c|c|c|c|c|c|c|}
\hline 8 & 1 & .00 & .29 & .00 & .00 & -.12 & .00 & .00 & .00 & .54 \\
\hline 9 & 6 & .00 & -.15 & -.25 & .00 & .03 & -.08 & .00 & -.09 & -.07 \\
\hline 10 & 1 & .00 & -.15 & -.25 & .00 & .35 & -.27 & .00 & -.29 & .04 \\
\hline 11 & 6 & .00 & -.14 & .25 & .00 & .03 & .08 & .00 & .09 & -.07 \\
\hline 12 & 1 & .00 & -.14 & .25 & .00 & .35 & .27 & .00 & .29 & .04 \\
\hline 13 & 1 & .00 & .00 & .00 & .04 & -.25 & .00 & .00 & .00 & -.01 \\
\hline 14 & 6 & .00 & .00 & .00 & .04 & .05 & .00 & .00 & .00 & .00 \\
\hline 15 & 1 & .00 & .00 & .00 & -.24 & -.05 & .00 & .00 & .00 & -.01 \\
\hline 16 & 1 & .00 & .00 & .00 & -.01 & -.01 & -.05 & -.01 & -.01 & .00 \\
\hline \multirow[t]{3}{*}{17} & 1 & .00 & .00 & .00 & -.01 & -.01 & .05 & .01 & .01 & .00 \\
\hline & & & 22 & & & 23 & & & 24 & \\
\hline & & & $A^{\prime}$ & & & A" & & \multirow{2}{*}{\multicolumn{3}{|c|}{1173.5073}} \\
\hline \multicolumn{2}{|c|}{ Frequencies } & -- & \multicolumn{2}{|c|}{1062.5826} & \multicolumn{3}{|c|}{1128.0695} & & & \\
\hline Red. & masses & -- & 2.1420 & & \multicolumn{3}{|c|}{1.7873} & \multicolumn{3}{|c|}{1.0313} \\
\hline \multicolumn{2}{|c|}{ Frc consts } & -- & 1.4249 & & \multicolumn{3}{|c|}{1.3401} & \multicolumn{3}{|c|}{.8368} \\
\hline \multicolumn{2}{|c|}{ IR Inten } & -- & 3.2122 & & \multicolumn{3}{|c|}{3.0605} & \multicolumn{3}{|c|}{.0000} \\
\hline Atom & AN & $\mathrm{x}$ & $\mathrm{Y}$ & Z & $\mathrm{x}$ & $\mathrm{Y}$ & Z & $\mathrm{x}$ & $\mathrm{Y}$ & Z \\
\hline 1 & 6 & .00 & -.05 & .00 & .00 & .00 & .00 & .00 & .00 & .02 \\
\hline 2 & 1 & -.01 & -.06 & .00 & .00 & .00 & .01 & .00 & .00 & .41 \\
\hline 3 & 6 & .00 & .02 & -.04 & .00 & .00 & .00 & .00 & .02 & -.01 \\
\hline 4 & 1 & .00 & .18 & -.14 & .00 & -.01 & .00 & .00 & .35 & -.20 \\
\hline 5 & 6 & .00 & .01 & .04 & .00 & .00 & .00 & .00 & -.02 & -.0 \\
\hline 6 & 1 & .00 & .18 & .14 & .00 & .00 & .00 & .00 & -.35 & -.20 \\
\hline 7 & 6 & .00 & -.06 & .00 & .00 & .00 & .00 & .00 & .00 & .02 \\
\hline 8 & 1 & .01 & -.06 & .00 & .00 & .00 & .02 & .00 & .00 & .41 \\
\hline 9 & 6 & .00 & .01 & -.04 & .00 & .00 & .00 & .00 & .02 & -.01 \\
\hline 10 & 1 & .00 & .18 & -.14 & .00 & .00 & .00 & .00 & .35 & -.20 \\
\hline 11 & 6 & .00 & .02 & .04 & .00 & .00 & .00 & .00 & -.02 & -.01 \\
\hline 12 & 1 & .00 & .18 & .14 & .00 & .01 & .00 & .00 & -.35 & -.20 \\
\hline 13 & 1 & -.09 & .60 & .00 & .00 & .00 & .45 & .00 & .00 & .00 \\
\hline 14 & 6 & -.09 & -.12 & .00 & .00 & .00 & -.18 & .00 & .00 & .00 \\
\hline 15 & 1 & .59 & .12 & .00 & .00 & .00 & .45 & .00 & .00 & .00 \\
\hline 16 & 1 & .03 & .03 & .12 & .28 & .40 & .20 & .00 & .00 & .00 \\
\hline \multirow[t]{3}{*}{17} & 1 & .03 & .03 & -.12 & -.28 & -.40 & .20 & .00 & .00 & .0 \\
\hline & & & 25 & & \multicolumn{3}{|c|}{26} & & 27 & \\
\hline & & & ?A & & & ?A & & & $A^{\prime}$ & \\
\hline Frequ & encies & -- & 1198.2927 & & & 98.293 & & & 74.3388 & \\
\hline Red. & masses & -- & 1.1288 & & & 1.128 & & & 1.2708 & \\
\hline Frc $C$ & onsts & -- & .9550 & & & .954 & & & 1.2159 & \\
\hline IR In & ten & -- & .0001 & & & .000 & & & 8.6325 & \\
\hline Atom & AN & $\mathrm{x}$ & Y & Z & $\mathrm{x}$ & $\mathrm{Y}$ & z & $\mathrm{x}$ & $\mathrm{Y}$ & Z \\
\hline 1 & 6 & .00 & .00 & .06 & .00 & .01 & .00 & .00 & .00 & .00 \\
\hline 2 & 1 & .00 & .00 & .57 & .00 & .00 & .00 & .00 & .00 & .00 \\
\hline 3 & 6 & .00 & .03 & -.01 & .00 & .04 & -.03 & .00 & .00 & .00 \\
\hline 4 & 1 & .00 & .25 & -.14 & .00 & .43 & -.25 & .00 & .00 & .0 \\
\hline 5 & 6 & .00 & .03 & .01 & .00 & -.04 & -.03 & .00 & .00 & .0 \\
\hline 6 & 1 & .00 & .25 & .14 & .00 & -.43 & -.25 & .00 & .00 & .0 \\
\hline 7 & 6 & .00 & .00 & -.06 & .00 & -.01 & .00 & .00 & .00 & .00 \\
\hline 8 & 1 & .00 & .00 & -.57 & .00 & .00 & .00 & .00 & .00 & .00 \\
\hline 9 & 6 & .00 & -.03 & .01 & .00 & -.04 & .03 & .00 & .00 & .0 \\
\hline 10 & 1 & .00 & -.25 & .14 & .00 & -.43 & .25 & .00 & .00 & .0 \\
\hline 11 & 6 & .00 & -.03 & -.01 & .00 & .04 & .03 & .00 & .00 & .0 \\
\hline 12 & 1 & .00 & -.25 & -.14 & .00 & .43 & .25 & .00 & .00 & .0 \\
\hline 13 & 1 & .00 & .00 & .00 & .00 & .00 & .00 & .10 & .20 & .00 \\
\hline 14 & 6 & .00 & .00 & .00 & .00 & .00 & .00 & .10 & -.07 & .0 \\
\hline 15 & 1 & .00 & .00 & .00 & .00 & .00 & .00 & -.15 & -.15 & .0 \\
\hline 16 & 1 & .00 & .00 & .00 & .00 & .00 & .00 & -.55 & .38 & .0 \\
\hline
\end{tabular}




\begin{tabular}{|c|c|c|c|c|c|c|c|c|c|c|}
\hline \multicolumn{2}{|l|}{17} & .00 & $\begin{array}{l}.00 \\
28 \\
\text { A" }\end{array}$ & .00 & \multicolumn{3}{|c|}{$\begin{array}{l}29 \\
\text { A" }\end{array}$} & -.55 & $\begin{array}{l}30^{.38} \\
\mathrm{Al}\end{array}$ & .00 \\
\hline \multicolumn{2}{|c|}{ Frequencies } & -- & \multicolumn{2}{|l|}{1360.8192} & \multicolumn{3}{|c|}{1367.3845} & \multicolumn{3}{|c|}{$\begin{array}{c}\text { A" } \\
1450.9534\end{array}$} \\
\hline \multicolumn{2}{|c|}{ Red. masses } & -- & \multicolumn{2}{|l|}{1.2123} & \multicolumn{3}{|c|}{1.2471} & \multicolumn{3}{|c|}{$\begin{array}{r}1450.9534 \\
9.4417\end{array}$} \\
\hline \multicolumn{2}{|c|}{ Frc consts } & -- & & & \multicolumn{3}{|c|}{1.3738} & \multicolumn{3}{|c|}{11.7114} \\
\hline \multicolumn{2}{|c|}{ IR Inten } & -- & 1.4286 & & & .000 & & & .0000 & \\
\hline Atom & AN & $\mathrm{x}$ & $\mathrm{Y}$ & Z & $\mathrm{x}$ & $\mathrm{Y}$ & Z & $\mathrm{x}$ & $\mathrm{Y}$ & Z \\
\hline 1 & 6 & .00 & .00 & .00 & .00 & .00 & -.06 & .00 & .00 & .36 \\
\hline 2 & 1 & .00 & .00 & .00 & .00 & .00 & .40 & .00 & .00 & -.20 \\
\hline 3 & 6 & .00 & .00 & .00 & .00 & .05 & -.03 & .00 & .31 & -.18 \\
\hline 4 & 1 & .00 & .00 & .00 & .00 & -.35 & .20 & .00 & -.17 & .10 \\
\hline 5 & 6 & .00 & .00 & .00 & .00 & .05 & .03 & .00 & -.31 & -.18 \\
\hline 6 & 1 & .00 & .00 & .00 & .00 & -.35 & -.20 & .00 & .17 & .10 \\
\hline 7 & 6 & .00 & .00 & .00 & .00 & .00 & .06 & .00 & .00 & .36 \\
\hline 8 & 1 & .00 & .00 & .01 & .00 & .00 & -.40 & .00 & .00 & -.20 \\
\hline 9 & 6 & .00 & .00 & .00 & .00 & -.05 & .03 & .00 & .31 & -.18 \\
\hline 10 & 1 & .00 & .00 & .00 & .00 & .35 & -.20 & .00 & -.17 & .10 \\
\hline 11 & 6 & .00 & .00 & .00 & .00 & -.05 & -.03 & .00 & -.31 & -.18 \\
\hline 12 & 1 & .00 & .00 & .00 & .00 & .35 & .20 & .00 & .17 & .10 \\
\hline 13 & 1 & .00 & .00 & .32 & .00 & .00 & .00 & .00 & .00 & .00 \\
\hline 14 & 6 & .00 & .00 & .00 & .00 & .00 & .00 & .00 & .00 & .00 \\
\hline 15 & 1 & .00 & .00 & -.32 & .00 & .00 & .00 & .00 & .00 & .00 \\
\hline 16 & 1 & .51 & -.37 & .00 & .00 & .00 & .00 & .00 & .00 & .00 \\
\hline 17 & 1 & -.51 & .37 & .00 & .00 & .00 & .00 & .00 & .00 & .00 \\
\hline & & & 31 & & & 32 & & & 33 & \\
\hline & & & $A^{\prime}$ & & & $A^{\prime \prime}$ & & & $A^{\prime}$ & \\
\hline Freq & dencies & -- & 1476.2085 & & & 04.786 & & & 504.7926 & \\
\hline Red. & masses & -- & 1.0702 & & & 2.014 & & & 2.0134 & \\
\hline FrC & consts & -- & 1.3740 & & & 2.687 & & & 2.6861 & \\
\hline IR I & nten & -- & 5.8589 & & & 7.309 & & & 7.4854 & \\
\hline Atom & AN & $\mathrm{X}$ & Y & Z & $\mathrm{x}$ & Y & Z & $\mathrm{X}$ & Y & Z \\
\hline 1 & 6 & .00 & .00 & .00 & .00 & .00 & .15 & .00 & .09 & .00 \\
\hline 2 & 1 & .00 & .00 & .00 & .00 & .00 & -.54 & .00 & .12 & .00 \\
\hline 3 & 6 & .00 & .00 & .00 & .00 & -.10 & -.03 & .00 & -.09 & .10 \\
\hline 4 & 1 & .00 & -.01 & .00 & .00 & .18 & -.22 & .00 & .43 & -.18 \\
\hline 5 & 6 & .00 & .00 & .00 & .00 & .10 & -.03 & .00 & -.09 & -.10 \\
\hline 6 & 1 & .00 & -.01 & .00 & .00 & -.18 & -.22 & .00 & .43 & .18 \\
\hline 7 & 6 & .00 & .00 & .00 & .00 & .00 & .15 & .00 & .09 & .00 \\
\hline 8 & 1 & .00 & .00 & .00 & .00 & .00 & -.54 & .00 & .12 & .00 \\
\hline 9 & 6 & .00 & .00 & .00 & .00 & -.10 & -.03 & .00 & -.09 & .10 \\
\hline 10 & 1 & .00 & -.01 & .00 & .00 & .18 & -.22 & .00 & .43 & -.18 \\
\hline 11 & 6 & .00 & .00 & .00 & .00 & .10 & -.03 & .00 & -.09 & -.10 \\
\hline 12 & 1 & .00 & -.01 & .00 & .00 & -.18 & -.22 & .00 & .43 & .18 \\
\hline 13 & 1 & -.03 & -.11 & .00 & .00 & .00 & .01 & .00 & -.01 & .00 \\
\hline 14 & 6 & -.03 & -.05 & .00 & .00 & .00 & .00 & .00 & .00 & .00 \\
\hline 15 & 1 & -.09 & -.07 & .00 & .00 & .00 & .00 & .00 & .00 & .00 \\
\hline 16 & 1 & .32 & .46 & .42 & .01 & .00 & .00 & .01 & .01 & .01 \\
\hline 17 & 1 & .32 & .46 & -.42 & -.01 & .00 & .00 & .01 & .01 & -.01 \\
\hline & & & 34 & & & 35 & & & 36 & \\
\hline & & & $A^{\prime}$ & & & $\mathrm{A}^{\prime \prime}$ & & & $A^{\prime}$ & \\
\hline Freq & dencies & -- & 1635.7060 & & & 35.707 & & & 270.1970 & \\
\hline Red. & masses & -- & 5.5549 & & & 5.555 & & & 2.1214 & \\
\hline Frc & consts & -- & 8.7566 & & & 8.756 & & & 6.4417 & \\
\hline IR I & hten & -- & .0001 & & & .000 & & & 2.8728 & \\
\hline Atom & AN & $\mathrm{x}$ & $\mathrm{Y}$ & Z & $\mathrm{x}$ & Y & Z & $\mathrm{X}$ & Y & Z \\
\hline 1 & 6 & .00 & .15 & .00 & .00 & .00 & .34 & .00 & .00 & .0 \\
\hline
\end{tabular}




\begin{tabular}{|c|c|c|c|c|c|c|c|c|c|c|}
\hline 2 & 1 & .00 & .17 & .00 & .00 & .00 & -.41 & .00 & .00 & .00 \\
\hline 3 & 6 & .00 & -.29 & .08 & .00 & .08 & -.20 & .00 & .00 & .00 \\
\hline 4 & 1 & .00 & .26 & -.25 & .00 & -.25 & -.03 & .00 & .00 & .00 \\
\hline 5 & 6 & .00 & .29 & .08 & .00 & .08 & .20 & .00 & .00 & .00 \\
\hline 6 & 1 & .00 & -.26 & -.25 & .00 & -.25 & .03 & .00 & .00 & .00 \\
\hline 7 & 6 & .00 & -.15 & .00 & .00 & .00 & -.34 & .00 & .00 & .00 \\
\hline 8 & 1 & .00 & -.17 & .00 & .00 & .00 & .41 & .00 & .00 & .00 \\
\hline 9 & 6 & .00 & .29 & -.08 & .00 & -.08 & .20 & .00 & .00 & .00 \\
\hline 10 & 1 & .00 & -.26 & .25 & .00 & .25 & .03 & .00 & .00 & .00 \\
\hline 11 & 6 & .00 & -.29 & -.08 & .00 & -.08 & -.20 & .00 & .00 & .0 \\
\hline 12 & 1 & .00 & .26 & .25 & .00 & .25 & -.03 & .00 & .00 & .0 \\
\hline 13 & 1 & .00 & .00 & .00 & .00 & .00 & .00 & .63 & .00 & .0 \\
\hline 14 & 6 & .00 & .00 & .00 & .00 & .00 & .00 & -.06 & -.11 & .00 \\
\hline 15 & 1 & .00 & .00 & .00 & .00 & .00 & .00 & -.25 & .70 & .00 \\
\hline 16 & 1 & .00 & .00 & .00 & .00 & .00 & .00 & -.04 & -.07 & .1 \\
\hline \multirow[t]{3}{*}{17} & 1 & .00 & .00 & .00 & .00 & .00 & .00 & -.04 & -.07 & -.1 \\
\hline & & & 37 & & & 38 & & & 39 & \\
\hline & & & $A^{\prime}$ & & & $A^{\prime}$ & & & $A^{\prime}$ & \\
\hline \multicolumn{2}{|c|}{ Frequencies } & -- & 2380.6745 & & \multicolumn{3}{|c|}{3139.9947} & \multicolumn{3}{|c|}{3195.5720} \\
\hline Red. & masses & -- & 2.3636 & & \multicolumn{3}{|c|}{1.0663} & \multicolumn{3}{|c|}{1.0839} \\
\hline \multicolumn{2}{|c|}{ Frc consts } & -- & 7.8925 & & \multicolumn{3}{|c|}{6.1941} & \multicolumn{3}{|c|}{6.5215} \\
\hline \multicolumn{2}{|c|}{ IR Inten } & -- & 4.0407 & & \multicolumn{3}{|c|}{12.4313} & \multicolumn{3}{|c|}{.0000} \\
\hline Atom & AN & $x$ & $\mathrm{Y}$ & Z & $\mathrm{x}$ & $\mathrm{Y}$ & Z & $\mathrm{x}$ & Y & Z \\
\hline 1 & 6 & .00 & .00 & .00 & .00 & .00 & .00 & .00 & -.03 & .00 \\
\hline 2 & 1 & .00 & .00 & .00 & .00 & .00 & .00 & .00 & .41 & .0 \\
\hline 3 & 6 & .00 & .00 & .00 & .00 & .00 & .00 & .00 & .02 & .0 \\
\hline 4 & 1 & .00 & .00 & .00 & .00 & .00 & .00 & .00 & -.20 & -.3 \\
\hline 5 & 6 & .00 & .00 & .00 & .00 & .00 & .00 & .00 & .02 & -.0 \\
\hline 6 & 1 & .00 & .00 & .00 & .00 & .00 & .00 & .00 & -.20 & .3 \\
\hline 7 & 6 & .00 & .00 & .00 & .00 & .00 & .00 & .00 & -.03 & .0 \\
\hline 8 & 1 & .00 & .00 & .00 & .00 & .00 & .00 & .00 & .41 & .0 \\
\hline 9 & 6 & .00 & .00 & .00 & .00 & .00 & .00 & .00 & .02 & .0 \\
\hline 10 & 1 & .00 & .00 & .00 & .00 & .00 & .00 & .00 & -.20 & -.3 \\
\hline 11 & 6 & .00 & .00 & .00 & .00 & .00 & .00 & .00 & .02 & -.0 \\
\hline 12 & 1 & .00 & .00 & .00 & .00 & .00 & .00 & .00 & -.20 & .3 \\
\hline 13 & 1 & .75 & .00 & .00 & -.03 & .01 & .00 & .00 & .00 & .0 \\
\hline 14 & 6 & -.16 & .10 & .00 & .04 & .06 & .00 & .00 & .00 & .0 \\
\hline 15 & 1 & .21 & -.59 & .00 & .02 & -.03 & .00 & .00 & .00 & .0 \\
\hline 16 & 1 & -.01 & .00 & .01 & -.23 & -.32 & .58 & .00 & .00 & .0 \\
\hline \multirow[t]{3}{*}{17} & 1 & -.01 & .00 & -.01 & -.23 & -.32 & -.58 & .00 & .00 & .0 \\
\hline & & & 40 & & & 41 & & & 42 & \\
\hline & & & A" & & & A" & & & $A^{\prime}$ & \\
\hline \multicolumn{2}{|c|}{ Frequencies } & -- & \multicolumn{2}{|l|}{3200.3064} & & 206.1042 & & & 206.1226 & \\
\hline Red. & masses & -- & 1.1020 & & & 1.0883 & & & 1.0883 & \\
\hline Frc & onsts & -- & 6.6500 & & & $6.590 \varepsilon$ & & & 6.5909 & \\
\hline IR Ir & ten & -- & 17.1762 & & & .0032 & & & .0003 & \\
\hline Atom & AN & $\mathrm{X}$ & $\mathrm{Y}$ & Z & $\mathrm{X}$ & $\mathrm{Y}$ & Z & $\mathrm{x}$ & $\mathrm{Y}$ & Z \\
\hline 1 & 6 & .00 & .00 & .00 & .00 & .00 & .00 & .00 & -.05 & .0 \\
\hline 2 & 1 & .00 & .00 & .00 & .00 & .00 & .00 & .00 & .58 & .0 \\
\hline 3 & 6 & .00 & .00 & .00 & .00 & .02 & .04 & .00 & .01 & .0 \\
\hline 4 & 1 & .00 & .00 & .00 & .00 & -.25 & -.43 & .00 & -.15 & -.2 \\
\hline 5 & 6 & .00 & .00 & .00 & .00 & .02 & -.04 & .00 & -.01 & .0 \\
\hline 6 & 1 & .00 & -.01 & .01 & .00 & -.25 & .43 & .00 & .15 & -.2 \\
\hline 7 & 6 & .00 & .00 & .00 & .00 & .00 & .00 & .00 & .05 & .0 \\
\hline 8 & 1 & .00 & .00 & .00 & .00 & .00 & .00 & .00 & -.57 & .0 \\
\hline 9 & 6 & .00 & .00 & .00 & .00 & -.02 & -.04 & .00 & -.01 & -.0 \\
\hline 10 & 1 & .00 & .01 & .01 & .00 & .25 & .43 & .00 & .15 & .2 \\
\hline
\end{tabular}




\begin{tabular}{|c|c|c|c|c|c|c|c|c|c|c|}
\hline 11 & 6 & .00 & .00 & .00 & .00 & -.02 & .04 & .00 & .01 & -.02 \\
\hline 12 & 1 & .00 & .00 & .00 & .00 & .25 & -.43 & .00 & -.15 & .25 \\
\hline 13 & 1 & .00 & .00 & .01 & .00 & .00 & .00 & .00 & .00 & .00 \\
\hline 14 & 6 & .00 & .00 & .09 & .00 & .00 & .00 & .00 & .00 & .00 \\
\hline 15 & 1 & .00 & .00 & .01 & .00 & .00 & .00 & .00 & .00 & .00 \\
\hline 16 & 1 & .24 & .34 & -.57 & .00 & .00 & .01 & .00 & .00 & \\
\hline \multirow[t]{3}{*}{17} & 1 & -.24 & -.34 & -.57 & .00 & .00 & .01 & .00 & .00 & .0 \\
\hline & & & 43 & & & 44 & & & 45 & \\
\hline & & & A" & & & $A^{\prime}$ & & & $A^{\prime}$ & \\
\hline \multicolumn{2}{|c|}{ Frequencies } & -- & 3221.9827 & & \multicolumn{3}{|c|}{3221.9986} & \multicolumn{3}{|c|}{3231.5111} \\
\hline \multicolumn{2}{|c|}{ Red. masses } & -- & 1.0964 & & \multicolumn{3}{|c|}{1.0964} & \multicolumn{3}{|c|}{1.1012} \\
\hline \multicolumn{2}{|c|}{ Frc consts } & -- & 6.7058 & & \multicolumn{3}{|c|}{6.7059} & \multicolumn{3}{|c|}{6.7755} \\
\hline \multicolumn{2}{|c|}{ IR Inten } & -- & 26.0995 & & \multicolumn{3}{|c|}{25.6250} & \multicolumn{3}{|c|}{.1074} \\
\hline Atom & AN & $\mathrm{x}$ & $\mathrm{Y}$ & $\mathrm{Z}$ & $\mathrm{X}$ & $\mathrm{Y}$ & Z & $\mathrm{x}$ & $\mathrm{Y}$ & Z \\
\hline 1 & 6 & .00 & .00 & .00 & .00 & -.05 & .00 & .00 & -.04 & . \\
\hline 2 & 1 & .00 & .00 & .00 & .00 & .57 & .00 & .00 & .41 & .00 \\
\hline 3 & 6 & .00 & -.02 & -.04 & .00 & -.01 & -.02 & .00 & -.02 & -.0 \\
\hline 4 & 1 & .00 & .25 & .43 & .00 & .14 & .25 & .00 & .20 & .3 \\
\hline 5 & 6 & .00 & .02 & -.04 & .00 & -.01 & .02 & .00 & .02 & -.0 \\
\hline 6 & 1 & .00 & -.25 & .43 & .00 & .14 & -.25 & .00 & -.20 & .3 \\
\hline 7 & 6 & .00 & .00 & .00 & .00 & -.05 & .00 & .00 & .04 & .0 \\
\hline 8 & 1 & .00 & .00 & .00 & .00 & .58 & .00 & .00 & -.41 & .0 \\
\hline 9 & 6 & .00 & -.02 & -.04 & .00 & -.01 & -.02 & .00 & .02 & .0 \\
\hline 10 & 1 & .00 & .25 & .43 & .00 & .14 & .25 & .00 & -.20 & -.3 \\
\hline 11 & 6 & .00 & .02 & -.04 & .00 & -.01 & .02 & .00 & -.02 & .0 \\
\hline 12 & 1 & .00 & -.25 & .43 & .00 & .14 & -.25 & .00 & .20 & -.3 \\
\hline 13 & 1 & .00 & .00 & .00 & .00 & .00 & .00 & .00 & .00 & .0 \\
\hline 14 & 6 & .00 & .00 & .00 & .00 & .00 & .00 & .00 & .00 & \\
\hline 15 & 1 & .00 & .00 & .00 & .00 & .00 & .00 & .00 & .00 & .0 \\
\hline 16 & 1 & .00 & .00 & .01 & .00 & .00 & .00 & .00 & .00 & .0 \\
\hline 17 & 1 & .00 & .00 & .01 & .00 & .00 & .00 & .00 & .00 & \\
\hline
\end{tabular}

- Thermochemistry -

Temperature 298.150 Kelvin. Pressure 1.00000 Atm.

Atom 1 has atomic number 6 and mass 12.00000

Atom 2 has atomic number 1 and mass 1.00783

Atom 3 has atomic number 6 and mass 12.00000

Atom 4 has atomic number 1 and mass 1.00783

Atom 5 has atomic number 6 and mass 12.00000

Atom 6 has atomic number 1 and mass 1.00783

Atom 7 has atomic number 6 and mass 12.00000

Atom 8 has atomic number 1 and mass 1.00783

Atom 9 has atomic number 6 and mass 12.00000

Atom 10 has atomic number 1 and mass 1.00783

Atom 11 has atomic number 6 and mass 12.00000

Atom 12 has atomic number 1 and mass 1.00783

Atom 13 has atomic number 1 and mass 2.01410

Atom 14 has atomic number 6 and mass 12.00000

Atom 15 has atomic number 1 and mass 2.01410

Atom 16 has atomic number 1 and mass 1.00783

Atom 17 has atomic number 1 and mass 1.00783

Molecular mass: $96.09080 \mathrm{amu}$.

Principal axes and moments of inertia in atomic units:

$$
\begin{array}{cccc}
\text { EIGENVALUES -- } & 652.582041023 .105041026 .45158 \\
X & .99944 & .03342 & .00000
\end{array}
$$




\begin{tabular}{|c|c|c|c|c|c|}
\hline-.0 & 342 & 9944 & 00000 & & \\
\hline .0 & 00 & 0000 & 00000 & & \\
\hline This molecule is an asymme & fic top. & & & & \\
\hline Rotational symmetry number & & & & & \\
\hline $\begin{array}{r}\text { Warning -- assumption of } \\
\text { may cause signif }\end{array}$ & $\begin{array}{l}\text { assical b } \\
\text { tant erro }\end{array}$ & $\begin{array}{l}\text { ehavior } f \\
r\end{array}$ & or rotati & & \\
\hline Rotational temperatures (K & (vin) & .13272 & .08 & 466 & .08438 \\
\hline Rotational constants (GHZ) & & 2.76554 & 1.76 & 398 & 1.75823 \\
\hline Zero-point vibrational ene & & $6262.3(\mathrm{~J}$ & oules/Mol & & \\
\hline & & $.53878)(\mathrm{K}$ & $\mathrm{Cal} / \mathrm{Mol}$ ) & & \\
\hline $\begin{array}{r}\text { Warning - } \text { explicit consid } \\
\text { vibrations may c }\end{array}$ & $\begin{array}{l}\text { lation of } \\
\text { ise signi }\end{array}$ & $\begin{array}{l}11 \text { degr } \\
\text { ficant er }\end{array}$ & $\begin{array}{l}\text { ees of } \mathrm{fr} \\
\text { ror }\end{array}$ & eedom as & \\
\hline Vibrational temperatures: & 30.48 & 57.64 & 60.20 & 114.74 & 128.10 \\
\hline (Kelvin) & 135.26 & 531.81 & 531.81 & 675.94 & 875.90 \\
\hline & 875.90 & 959.10 & 1200.42 & 1200.44 & 1291.27 \\
\hline & 1291.28 & 1334.69 & 1451.05 & 1454.85 & 1523.39 \\
\hline & 1524.28 & 1528.82 & 1623.04 & 1688.41 & 1724.07 \\
\hline & 1724.07 & 1833.49 & 1957.91 & 1967.36 & 2087.60 \\
\hline & 2123.93 & 2165.05 & 2165.06 & 2353.41 & 2353.42 \\
\hline & 3266.30 & 3425.26 & 4517.75 & 4597.71 & 4604.52 \\
\hline & 4612.86 & 4612.89 & 4635.71 & 4635.73 & 4649.42 \\
\hline Zero-point correction= & & & .13 & 502 ( Ha & tree/Par \\
\hline
\end{tabular}


XXIV. Harmonic frequency analysis and zero-point energy for 1,2,3-trideuterated methane-benzene complex with the MP2/6-311++G(d,p) method:

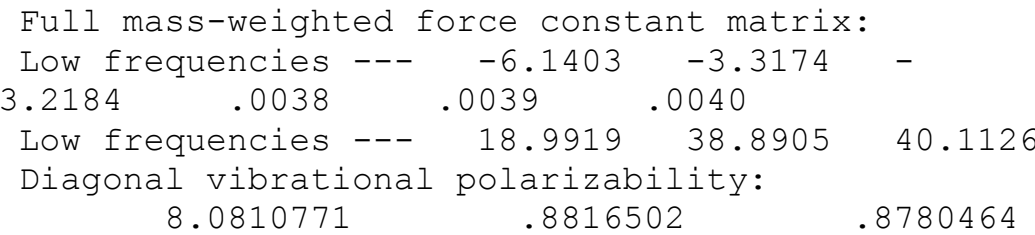

Harmonic frequencies ( $\left.\mathrm{cm}^{*} *-1\right)$, IR intensities (KM/Mole), Raman scattering activities ( $A^{* * 4 / A M U), ~ d e p o l a r i z a t i o n ~ r a t i o s ~ f o r ~ p l a n e ~ a n d ~ u n p o l a r i z e d ~}$ incident light, reduced masses (AMU), force constants (mDyne/A), and normal coordinates:

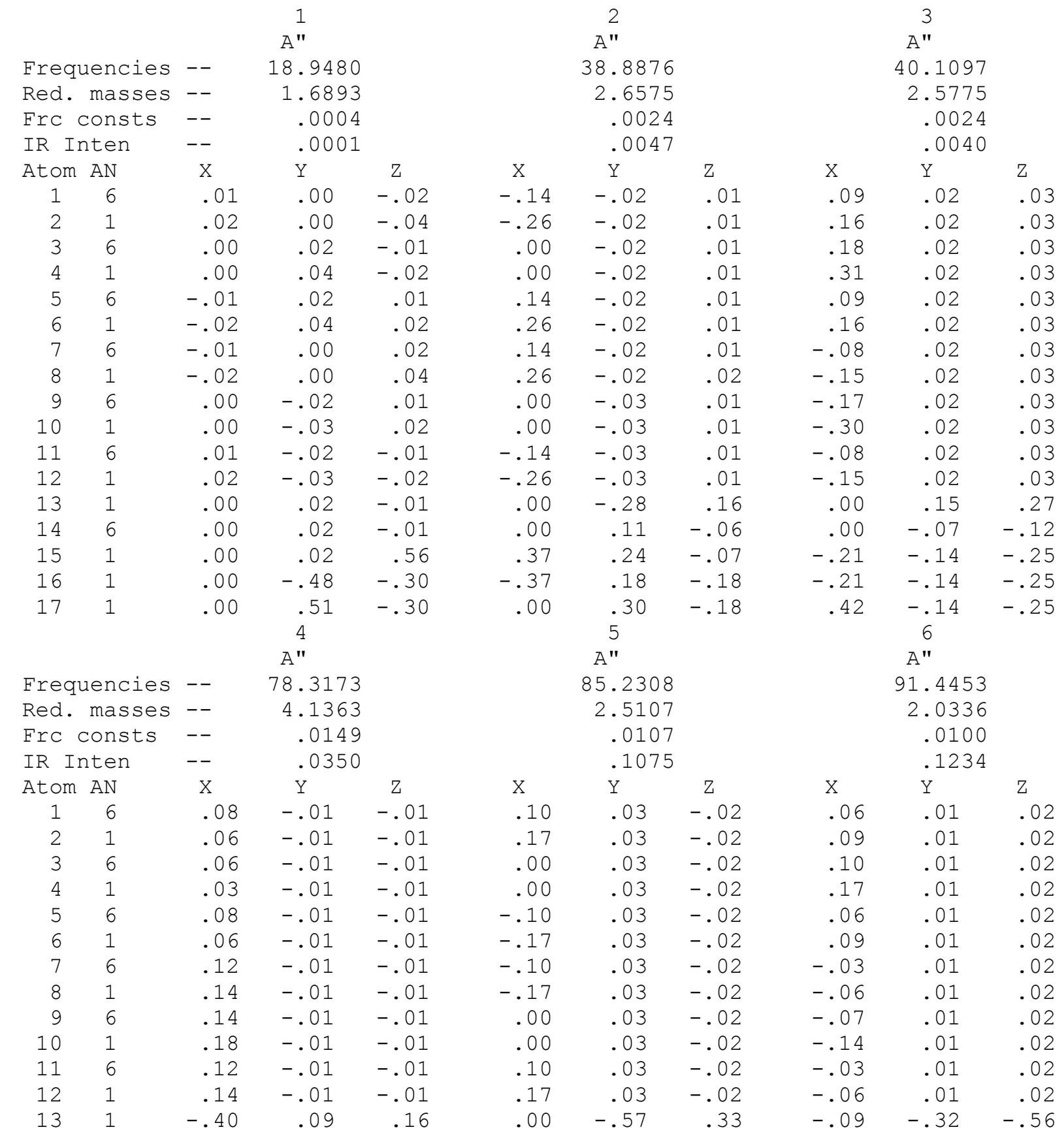




\begin{tabular}{|c|c|c|c|c|c|c|c|c|c|c|}
\hline 14 & 6 & -.40 & .03 & .04 & .00 & -.12 & .07 & -.09 & -.05 & -.09 \\
\hline 15 & 1 & -.46 & .00 & .01 & .43 & .04 & -.01 & .17 & .04 & .07 \\
\hline 16 & 1 & -.46 & .00 & .01 & -.43 & .03 & -.02 & .17 & .04 & .07 \\
\hline \multirow[t]{2}{*}{17} & 1 & -.28 & .00 & .01 & .00 & $\dot{8}^{.04}$ & -.02 & -.61 &.$_{9}^{.04}$ & .07 \\
\hline & & & ?A & & & ?A & & \multicolumn{3}{|c|}{$A^{\prime}$} \\
\hline \multicolumn{2}{|c|}{ Frequencies } & -- & \multicolumn{2}{|l|}{369.6277} & \multicolumn{3}{|c|}{369.6288} & \multicolumn{3}{|c|}{469.8032} \\
\hline \multicolumn{2}{|c|}{ Red. masses } & -- & \multicolumn{2}{|l|}{3.4123} & \multicolumn{3}{|c|}{3.4124} & \multicolumn{3}{|c|}{9.9359} \\
\hline \multicolumn{2}{|c|}{ Frc consts } & -- & \multicolumn{2}{|l|}{.2747} & \multicolumn{3}{|c|}{.2747} & \multicolumn{3}{|c|}{1.2921} \\
\hline \multicolumn{2}{|c|}{ IR Inten } & -- & .0004 & & \multicolumn{3}{|c|}{.0004} & & .0000 & \\
\hline Atom & AN & X & $\mathrm{Y}$ & Z & $\mathrm{X}$ & $\mathrm{Y}$ & $\mathrm{Z}$ & $\mathrm{X}$ & $\mathrm{Y}$ & Z \\
\hline 1 & 6 & .27 & .00 & .00 & .01 & .00 & .00 & .37 & .00 & .00 \\
\hline 2 & 1 & .51 & .00 & .00 & .01 & .00 & .00 & .18 & .00 & .00 \\
\hline 3 & 6 & -.14 & .00 & .00 & .23 & .00 & .00 & -.37 & .00 & .00 \\
\hline 4 & 1 & -.27 & .00 & .00 & .44 & .00 & .00 & -.18 & .00 & .00 \\
\hline 5 & 6 & -.13 & .00 & .00 & -.24 & .00 & .00 & .37 & .00 & .00 \\
\hline 6 & 1 & -.25 & .00 & .00 & -.44 & .00 & .00 & .18 & .00 & .00 \\
\hline 7 & 6 & .27 & .00 & .00 & .01 & .00 & .00 & -.37 & .00 & .00 \\
\hline 8 & 1 & .51 & .00 & .00 & .01 & .00 & .00 & -.18 & .00 & .00 \\
\hline 9 & 6 & -.14 & .00 & .00 & .23 & .00 & .00 & .37 & .00 & .00 \\
\hline 10 & 1 & -.26 & .00 & .00 & .43 & .00 & .00 & .18 & .00 & .00 \\
\hline 11 & 6 & -.13 & .00 & .00 & -.24 & .00 & .00 & -.37 & .00 & .00 \\
\hline 12 & 1 & -.25 & .00 & .00 & -.45 & .00 & .00 & -.18 & .00 & .00 \\
\hline 13 & 1 & .00 & .00 & .00 & .00 & .00 & .00 & .00 & .00 & .00 \\
\hline 14 & 6 & .00 & .00 & .00 & .00 & .00 & .00 & .00 & .00 & .00 \\
\hline 15 & 1 & .00 & .00 & .00 & .00 & .00 & .00 & .00 & .00 & .00 \\
\hline 16 & 1 & .00 & .00 & .00 & .00 & .00 & .00 & .00 & .00 & .00 \\
\hline 17 & 1 & .00 & .00 & .00 & .00 & .00 & .00 & .00 & .00 & .00 \\
\hline & & & 10 & & & 11 & & & 12 & \\
\hline & & & ?A & & & ?A & & & $A^{\prime}$ & \\
\hline Frequ & dencies & -- & 608.7809 & & & 608.7815 & & & 666.6101 & \\
\hline Red. & masses & -- & 6.0129 & & & 6.0129 & & & 1.0850 & \\
\hline Frc $C$ & consts & -- & 1.3130 & & & 1.3130 & & & .2841 & \\
\hline IR In & tten & -- & .0000 & & & .0000 & & & 127.8678 & \\
\hline Atom & AN & X & Y & Z & $\mathrm{X}$ & Y & Z & $\mathrm{X}$ & $\mathrm{Y}$ & Z \\
\hline 1 & 6 & .00 & .00 & .15 & .00 & .36 & .00 & -.03 & .00 & .00 \\
\hline 2 & 1 & .00 & .00 & -.23 & -.01 & .36 & .00 & .41 & .00 & .00 \\
\hline 3 & 6 & .00 & .22 & .23 & .00 & .02 & -.22 & -.03 & .00 & .00 \\
\hline 4 & 1 & -.01 & .05 & .33 & .00 & -.26 & -.06 & .40 & .00 & .00 \\
\hline 5 & 6 & .00 & .22 & -.23 & .00 & -.03 & -.22 & -.03 & .00 & .00 \\
\hline 6 & 1 & .01 & .05 & -.33 & .00 & .26 & -.05 & .41 & .00 & .00 \\
\hline 7 & 6 & .00 & .00 & -.15 & .00 & -.36 & .00 & -.03 & .00 & .00 \\
\hline 8 & 1 & .00 & .00 & .23 & -.01 & -.36 & .00 & .40 & .00 & .00 \\
\hline 9 & 6 & .00 & -.22 & -.23 & .00 & -.02 & .22 & -.03 & .00 & .00 \\
\hline 10 & 1 & -.01 & -.05 & -.33 & .00 & .26 & .06 & .41 & .00 & .00 \\
\hline 11 & 6 & .00 & -.22 & .23 & .00 & .03 & .22 & -.03 & .00 & .00 \\
\hline 12 & 1 & .01 & -.05 & .33 & .00 & -.26 & .05 & .40 & .00 & .00 \\
\hline 13 & 1 & .00 & .00 & .00 & .00 & .00 & .00 & .00 & .00 & .00 \\
\hline 14 & 6 & .00 & .00 & .00 & .00 & .00 & .00 & .00 & .00 & .00 \\
\hline 15 & 1 & .00 & .00 & .00 & .00 & .00 & .00 & .00 & .00 & .00 \\
\hline 16 & 1 & .00 & .00 & .00 & .00 & .00 & .00 & .00 & .00 & .00 \\
\hline 17 & 1 & .00 & .00 & .00 & .00 & .00 & .00 & .00 & .00 & .00 \\
\hline & & & 13 & & & 14 & & & 15 & \\
\hline Fregu & dencies & -- & $\begin{array}{c}\text { ?A } \\
834.3348\end{array}$ & & & $\begin{array}{c}\text { ?A } \\
834.3444\end{array}$ & & & $\begin{array}{c}\text { ?A } \\
897.4793\end{array}$ & \\
\hline Red. & masses & -- & 1.2494 & & & 1. 2494 & & & 1.2780 & \\
\hline Frc $C$ & zonsts & -- & .5124 & & & .5124 & & & .6065 & \\
\hline
\end{tabular}




\begin{tabular}{|c|c|c|c|c|c|c|c|c|c|c|}
\hline IR I & nten & -- & .133 & & & .134 & & & .0014 & \\
\hline Atom & AN & $\mathrm{x}$ & $\mathrm{Y}$ & Z & $\mathrm{X}$ & $\mathrm{Y}$ & Z & $\mathrm{x}$ & $\mathrm{Y}$ & Z \\
\hline 1 & 6 & -.09 & .00 & .00 & .00 & .00 & .00 & -.09 & .00 & .00 \\
\hline 2 & 1 & .60 & .00 & .00 & -.03 & .00 & .00 & .54 & .00 & .00 \\
\hline 3 & 6 & -.04 & .00 & .00 & .07 & .00 & .00 & .05 & .00 & .00 \\
\hline 4 & 1 & .24 & .00 & .00 & -.48 & .00 & .00 & -.30 & .00 & .00 \\
\hline 5 & 6 & .05 & .00 & .00 & .08 & .00 & .00 & .04 & .00 & .00 \\
\hline 6 & 1 & -.33 & .00 & .00 & -.50 & .00 & .00 & -.27 & .00 & .00 \\
\hline 7 & 6 & .08 & .00 & .00 & .00 & .00 & .00 & -.09 & .00 & .00 \\
\hline 8 & 1 & -.54 & .00 & .00 & .03 & .00 & .00 & .60 & .00 & .00 \\
\hline 9 & 6 & .04 & .00 & .00 & -.08 & .00 & .00 & .04 & .00 & .00 \\
\hline 10 & 1 & -.27 & .00 & .00 & .54 & .00 & .00 & -.27 & .00 & .00 \\
\hline 11 & 6 & -.04 & .00 & .00 & -.07 & .00 & .00 & .05 & .00 & .00 \\
\hline 12 & 1 & .30 & .00 & .00 & .45 & .00 & .00 & -.30 & .00 & .00 \\
\hline 13 & 1 & .00 & .01 & .00 & .00 & .00 & -.01 & .00 & .00 & .00 \\
\hline 14 & 6 & .00 & .00 & .00 & .00 & .00 & .00 & .00 & .00 & .00 \\
\hline 15 & 1 & .00 & .00 & .00 & .00 & .00 & .00 & .00 & .00 & .00 \\
\hline 16 & 1 & .00 & .00 & .00 & .00 & .00 & .00 & .00 & .00 & .00 \\
\hline 17 & 1 & .00 & .00 & .00 & .00 & .00 & .00 & .00 & .00 & .00 \\
\hline & & & 16 & & & 17 & & & 18 & \\
\hline & & & ?A & & & $A^{\prime}$ & & & A" & \\
\hline Freq & uencies & -- & 897.487 & & & .7 .656 & & & 008.5241 & \\
\hline Red. & masses & -- & 1.278 & & & 1.025 & & & 5.9676 & \\
\hline Frc & consts & -- & .606 & & & .520 & & & 3.5762 & \\
\hline IR I & nten & -- & .001 & & & .003 & & & .0241 & \\
\hline Atom & AN & $\mathrm{x}$ & $\mathrm{Y}$ & Z & $\mathrm{x}$ & $\mathrm{Y}$ & Z & $\mathrm{x}$ & $\mathrm{Y}$ & Z \\
\hline 1 & 6 & .00 & .00 & .00 & .02 & .00 & .00 & .00 & .27 & .00 \\
\hline 2 & 1 & .00 & .00 & .00 & -.41 & .00 & .00 & .00 & .30 & .00 \\
\hline 3 & 6 & -.08 & .00 & .00 & -.02 & .00 & .00 & .00 & .14 & .24 \\
\hline 4 & 1 & .52 & .00 & .00 & .41 & .00 & .00 & .00 & .15 & .26 \\
\hline 5 & 6 & .07 & .00 & .00 & .02 & .00 & .00 & .00 & -.14 & .24 \\
\hline 6 & 1 & -.47 & .00 & .00 & -.41 & .00 & .00 & .00 & -.15 & .26 \\
\hline 7 & 6 & .00 & .00 & .00 & -.02 & .00 & .00 & .00 & -.28 & .00 \\
\hline 8 & 1 & .00 & .00 & .00 & .41 & .00 & .00 & .00 & -.30 & .00 \\
\hline 9 & 6 & -.07 & .00 & .00 & .02 & .00 & .00 & .00 & -.14 & -.24 \\
\hline 10 & 1 & .47 & .00 & .00 & -.41 & .00 & .00 & .00 & -.15 & -.26 \\
\hline 11 & 6 & .08 & .00 & .00 & -.02 & .00 & .00 & .00 & .14 & -.24 \\
\hline 12 & 1 & -.52 & .00 & .00 & .41 & .00 & .00 & .00 & .15 & -.26 \\
\hline 13 & 1 & .00 & .00 & .00 & .00 & .00 & .00 & .00 & -.01 & -.01 \\
\hline 14 & 6 & .00 & .00 & .00 & .00 & .00 & .00 & .00 & .00 & .00 \\
\hline 15 & 1 & .00 & .00 & .00 & .00 & .00 & .00 & -.01 & .00 & .00 \\
\hline 16 & 1 & .00 & .00 & .00 & .00 & .00 & .00 & -.01 & .00 & .00 \\
\hline 17 & 1 & .00 & .00 & .00 & .00 & .00 & .00 & -.01 & .00 & .00 \\
\hline & & & 19 & & & 20 & & & 21 & \\
\hline & & & $A^{\prime}$ & & & A" & & & A" & \\
\hline Freq & uencies & -- & 1011.172 & & & 6.768 & & & 059.0482 & \\
\hline Red. & masses & -- & 6.536 & & & 2.498 & & & 1.7254 & \\
\hline Frc & consts & -- & 3.937 & & & 1.582 & & & 1.1402 & \\
\hline IR I & nten & -- & .000 & & & 2.583 & & & 3.5692 & \\
\hline Atom & AN & $\mathrm{x}$ & Y & Z & $\mathrm{X}$ & Y & Z & $\mathrm{X}$ & Y & Z \\
\hline 1 & 6 & .00 & .29 & .00 & .00 & .01 & -.01 & .00 & -.10 & -.04 \\
\hline 2 & 1 & .00 & .29 & .00 & .00 & .01 & -.03 & .00 & -.11 & -.27 \\
\hline 3 & 6 & .00 & -.14 & -.25 & .00 & .00 & .01 & .00 & .07 & -.04 \\
\hline 4 & 1 & .00 & -.14 & -.25 & -.01 & .01 & .01 & .00 & .46 & -.26 \\
\hline 5 & 6 & .00 & -.15 & .25 & .00 & -.01 & .00 & .00 & -.02 & .11 \\
\hline 6 & 1 & .00 & -.15 & .25 & .00 & -.03 & -.01 & .00 & .17 & .23 \\
\hline 7 & 6 & .00 & .29 & .00 & .00 & .00 & .00 & .00 & -.10 & -.04 \\
\hline
\end{tabular}




\begin{tabular}{|c|c|c|c|c|c|c|c|c|c|c|}
\hline 8 & 1 & .00 & .29 & .00 & .00 & .00 & -.03 & .00 & -.11 & -.27 \\
\hline 9 & 6 & .00 & -.15 & -.25 & .00 & .00 & .00 & .00 & .07 & -.04 \\
\hline 10 & 1 & .00 & -.15 & -.25 & .00 & .00 & .01 & .00 & .46 & -.27 \\
\hline 11 & 6 & .00 & -.14 & .25 & .00 & -.01 & .00 & .00 & -.02 & .11 \\
\hline 12 & 1 & .00 & -.14 & .25 & .00 & -.02 & -.01 & .00 & .17 & .23 \\
\hline 13 & 1 & .00 & .00 & .00 & -.08 & .28 & .48 & .00 & -.14 & .08 \\
\hline 14 & 6 & .00 & .00 & .00 & -.08 & -.11 & -.19 & .00 & .03 & -.02 \\
\hline 15 & 1 & .00 & .00 & .00 & .29 & .03 & .46 & -.09 & .00 & .01 \\
\hline 16 & 1 & .00 & .00 & .00 & .29 & .38 & .25 & .09 & -.01 & .00 \\
\hline \multirow[t]{3}{*}{17} & 1 & .00 & .00 & .00 & -.05 & -.09 & -.16 & .00 & -.04 & .02 \\
\hline & & & 22 & & & 23 & & & 24 & \\
\hline & & & $\mathrm{A}^{\prime \prime}$ & & & A" & & \multirow{2}{*}{\multicolumn{3}{|c|}{1065.8905}} \\
\hline \multicolumn{2}{|c|}{ Frequencies } & -- & \multicolumn{2}{|c|}{1059.4596} & \multicolumn{3}{|c|}{1060.7709} & & & \\
\hline \multicolumn{2}{|c|}{ Red. masses } & -- & 1.7156 & & \multicolumn{3}{|c|}{2.1875} & \multicolumn{3}{|c|}{2.1727} \\
\hline \multirow{2}{*}{\multicolumn{2}{|c|}{$\begin{array}{l}\text { Frc consts } \\
\text { IR Inten }\end{array}$}} & -- & 1.1346 & & \multicolumn{3}{|c|}{1.4502} & \multicolumn{3}{|c|}{1.4543} \\
\hline & & -- & 5.7848 & & \multicolumn{3}{|c|}{4.4931} & \multicolumn{3}{|c|}{1.5410} \\
\hline \multicolumn{2}{|c|}{$\begin{array}{l}\text { IR Inten } \\
\text { Atom AN }\end{array}$} & $\mathrm{x}$ & $\mathrm{Y}$ & Z & $\mathrm{x}$ & $\mathrm{Y}$ & Z & $\mathrm{x}$ & Y & Z \\
\hline 1 & 6 & .00 & -.06 & .07 & .00 & .01 & -.02 & .00 & -.03 & -.01 \\
\hline 2 & 1 & .00 & -.06 & .46 & .00 & .01 & -.11 & .00 & -.03 & -.08 \\
\hline 3 & 6 & .00 & -.06 & -.10 & .00 & .01 & .02 & .00 & .02 & -.01 \\
\hline 4 & 1 & .00 & -.06 & -.11 & .00 & .01 & .02 & .00 & .13 & -.08 \\
\hline 5 & 6 & .00 & .09 & -.01 & .00 & -.02 & .00 & .00 & .00 & .03 \\
\hline 6 & 1 & .00 & .43 & .18 & .00 & -.10 & -.04 & .00 & .05 & .0 \\
\hline 7 & 6 & .00 & -.06 & .07 & .00 & .02 & -.02 & .00 & -.03 & -.01 \\
\hline 8 & 1 & .00 & -.06 & .46 & .00 & .02 & -.10 & .01 & -.03 & -.08 \\
\hline 9 & 6 & .00 & -.06 & -.10 & .00 & .01 & .02 & .00 & .02 & -.01 \\
\hline 10 & 1 & .00 & -.06 & -.11 & .00 & .02 & .03 & .00 & .13 & -.08 \\
\hline 11 & 6 & .00 & .09 & -.01 & .00 & -.02 & .01 & .00 & .00 & .0 \\
\hline 12 & 1 & .00 & .43 & .18 & .00 & -.10 & -.04 & -.01 & .05 & .0 \\
\hline 13 & 1 & .02 & .00 & .00 & .14 & -.08 & -.14 & .00 & .63 & -.36 \\
\hline 14 & 6 & .02 & -.01 & -.02 & .15 & -.03 & -.05 & .00 & -.13 & .08 \\
\hline 15 & 1 & -.07 & -.04 & .09 & -.45 & -.23 & .40 & .40 & .02 & -.05 \\
\hline 16 & 1 & -.07 & .10 & .01 & -.45 & .46 & .00 & -.40 & .05 & .0 \\
\hline \multirow[t]{3}{*}{17} & 1 & -.04 & .00 & .00 & -.22 & .04 & .06 & .00 & .18 & -.1 \\
\hline & & & 25 & & & 26 & & & 27 & \\
\hline & & & A" & & & ?A & & & ?A & \\
\hline Frequ & encies & -- & 1173.5072 & & & 98.290 & & & 198.2942 & \\
\hline Red. & masses & -- & 1.0313 & & & 1.128 & & & 1.1288 & \\
\hline Frc & onsts & -- & .8368 & & & .954 & & & .9549 & \\
\hline IR Ir & ten & -- & .0000 & & & .000 & & & .0001 & \\
\hline Atom & AN & $\mathrm{X}$ & Y & Z & $\mathrm{x}$ & $\mathrm{Y}$ & Z & $\mathrm{x}$ & Y & Z \\
\hline 1 & 6 & .00 & .00 & .02 & .00 & .00 & .05 & .00 & .00 & .0 \\
\hline 2 & 1 & .00 & .00 & .41 & .00 & .00 & .49 & .00 & .00 & .30 \\
\hline 3 & 6 & .00 & .02 & -.01 & .00 & .05 & -.02 & .00 & -.02 & .02 \\
\hline 4 & 1 & .00 & .35 & -.20 & .00 & .44 & -.25 & .00 & -.24 & .1 \\
\hline 5 & 6 & .00 & -.02 & -.01 & .00 & .00 & -.01 & .00 & .05 & .0 \\
\hline 6 & 1 & .00 & -.35 & -.20 & .00 & -.01 & -.01 & .00 & .50 & .2 \\
\hline 7 & 6 & .00 & .00 & .02 & .00 & .00 & -.05 & .00 & .00 & -.0 \\
\hline 8 & 1 & .00 & .00 & .41 & .00 & .00 & -.49 & .00 & .00 & -.30 \\
\hline 9 & 6 & .00 & .02 & -.01 & .00 & -.05 & .02 & .00 & .02 & -.02 \\
\hline 10 & 1 & .00 & .35 & -.20 & .00 & -.44 & .25 & .00 & .24 & -.1 \\
\hline 11 & 6 & .00 & -.02 & -.01 & .00 & .00 & .01 & .00 & -.05 & -.0 \\
\hline 12 & 1 & .00 & -.35 & -.20 & .00 & .01 & .01 & .00 & -.50 & -.2 \\
\hline 13 & 1 & .00 & .00 & .00 & .00 & .00 & .00 & .00 & .00 & .0 \\
\hline 14 & 6 & .00 & .00 & .00 & .00 & .00 & .00 & .00 & .00 & .0 \\
\hline 15 & 1 & .00 & .00 & .00 & .00 & .00 & .00 & .00 & .00 & .0 \\
\hline 16 & 1 & .00 & .00 & .00 & .00 & .00 & .00 & .00 & .00 & .0 \\
\hline
\end{tabular}




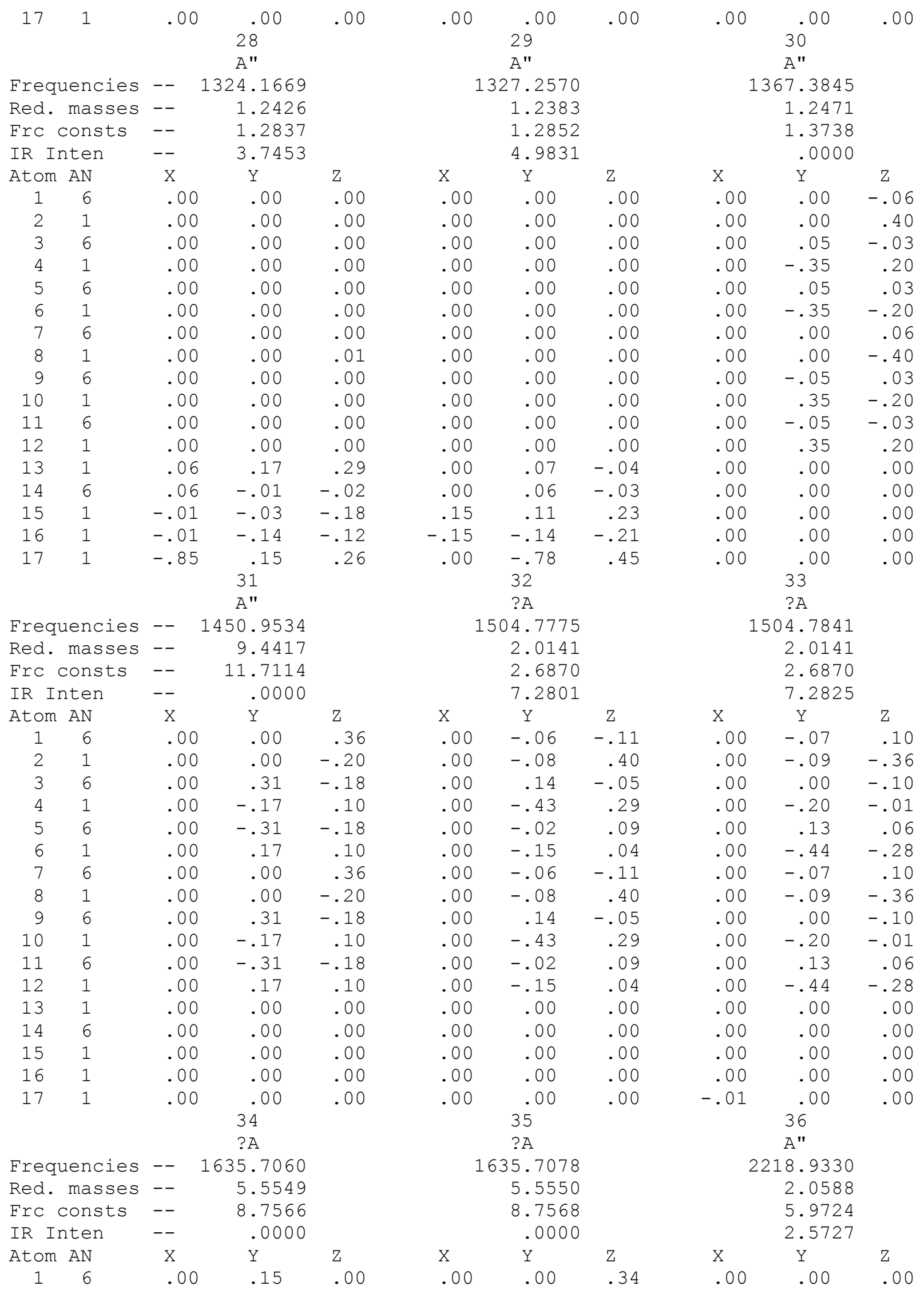




\begin{tabular}{|c|c|c|c|c|c|c|c|c|c|c|}
\hline 2 & 1 & .00 & .17 & .00 & .00 & .00 & -.41 & .00 & .00 & .00 \\
\hline 3 & 6 & .00 & -.29 & .08 & .00 & .08 & -.20 & .00 & .00 & .00 \\
\hline 4 & 1 & .00 & .26 & -.25 & .00 & -.25 & -.03 & .00 & .00 & .00 \\
\hline 5 & 6 & .00 & .29 & .08 & .00 & .08 & .20 & .00 & .00 & .00 \\
\hline 6 & 1 & .00 & -.26 & -.25 & .00 & -.25 & .03 & .00 & .00 & .00 \\
\hline 7 & 6 & .00 & -.15 & .00 & .00 & .00 & -.34 & .00 & .00 & .00 \\
\hline 8 & 1 & .00 & -.17 & .00 & .00 & .00 & .41 & .00 & .00 & .00 \\
\hline 9 & 6 & .00 & .29 & -.08 & .00 & -.08 & .20 & .00 & .00 & .00 \\
\hline 10 & 1 & .00 & -.26 & .25 & .00 & .25 & .03 & .00 & .00 & .00 \\
\hline 11 & 6 & .00 & -.29 & -.08 & .00 & -.08 & -.20 & .00 & .00 & .0 \\
\hline 12 & 1 & .00 & .26 & .25 & .00 & .25 & -.03 & .00 & .00 & .0 \\
\hline 13 & 1 & .00 & .00 & .00 & .00 & .00 & .00 & .53 & .00 & .0 \\
\hline 14 & 6 & .00 & .00 & .00 & .00 & .00 & .00 & -.02 & -.04 & -.07 \\
\hline 15 & 1 & .00 & .00 & .00 & .00 & .00 & .00 & -.19 & .55 & .00 \\
\hline 16 & 1 & .00 & .00 & .00 & .00 & .00 & .00 & -.19 & -.28 & .4 \\
\hline \multirow[t]{3}{*}{17} & 1 & .00 & .00 & .00 & .00 & .00 & .00 & -.05 & -.07 & -.1 \\
\hline & & & 37 & & & 38 & & & 39 & \\
\hline & & & A" & & & A" & & & A" & \\
\hline \multicolumn{2}{|c|}{ Frequencies } & -- & 2369.8218 & & \multicolumn{3}{|c|}{2383.6548} & \multicolumn{3}{|c|}{3171.3193} \\
\hline Red. & masses & -- & 2.3670 & & \multicolumn{3}{|c|}{2.3650} & \multicolumn{3}{|c|}{1.0862} \\
\hline \multicolumn{2}{|c|}{ Frc consts } & -- & 7.8321 & & \multirow{2}{*}{\multicolumn{3}{|c|}{$\begin{array}{l}7.9171 \\
2.9875\end{array}$}} & \multicolumn{3}{|c|}{6.4361} \\
\hline & -- & 8.4426 & & & & & \multicolumn{3}{|c|}{14.9583} \\
\hline \multicolumn{2}{|c|}{$\begin{array}{l}\text { IR Inten } \\
\text { Atom AN }\end{array}$} & $\mathrm{X}$ & $\mathrm{Y}$ & Z & $\mathrm{x}$ & $\mathrm{Y}$ & Z & $\mathrm{x}$ & $\mathrm{Y}$ & Z \\
\hline 1 & 6 & .00 & .00 & .00 & .00 & .00 & .00 & .00 & .00 & .00 \\
\hline 2 & 1 & .00 & .00 & .00 & .00 & .00 & .00 & .00 & .00 & .0 \\
\hline 3 & 6 & .00 & .00 & .00 & .00 & .00 & .00 & .00 & .00 & .00 \\
\hline 4 & 1 & .00 & .00 & .00 & .00 & .00 & .00 & .00 & .00 & .0 \\
\hline 5 & 6 & .00 & .00 & .00 & .00 & .00 & .00 & .00 & .00 & .0 \\
\hline 6 & 1 & .00 & .00 & .00 & .00 & .00 & .00 & .00 & .00 & .0 \\
\hline 7 & 6 & .00 & .00 & .00 & .00 & .00 & .00 & .00 & .00 & .0 \\
\hline 8 & 1 & .00 & .00 & .00 & .00 & .00 & .00 & .00 & .00 & .0 \\
\hline 9 & 6 & .00 & .00 & .00 & .00 & .00 & .00 & .00 & .00 & .00 \\
\hline 10 & 1 & .00 & .00 & .00 & .00 & .00 & .00 & .00 & .00 & .0 \\
\hline 11 & 6 & .00 & .00 & .00 & .00 & .00 & .00 & .00 & .00 & .0 \\
\hline 12 & 1 & .00 & .00 & .00 & .00 & .00 & .00 & .00 & .00 & .0 \\
\hline 13 & 1 & .00 & .00 & .00 & .83 & .00 & .00 & -.02 & .00 & .0 \\
\hline 14 & 6 & .00 & .16 & -.09 & -.18 & .03 & .05 & .03 & .04 & .0 \\
\hline 15 & 1 & .23 & -.65 & .00 & .12 & -.35 & .00 & .01 & -.02 & .0 \\
\hline 16 & 1 & -.23 & -.32 & .57 & .12 & .17 & -.30 & .01 & .01 & -.0 \\
\hline \multirow[t]{3}{*}{17} & 1 & .00 & .01 & -.01 & -.01 & .00 & -.01 & -.33 & -.47 & -.8 \\
\hline & & & 40 & & & 41 & & & 42 & \\
\hline & & & $A^{\prime}$ & & & A" & & & $A^{\prime}$ & \\
\hline \multirow{2}{*}{\multicolumn{2}{|c|}{$\begin{array}{l}\text { Frequencies } \\
\text { Red masses }\end{array}$}} & -- & \multicolumn{2}{|l|}{3195.5720} & & 06.103 & & & 206.1226 & \\
\hline Red. & & -- & 1.0839 & & & 1.088 & & & 1.0883 & \\
\hline Frc & onsts & -- & 6.5215 & & & 6.590 & & & 6.5909 & \\
\hline IR Ir & ten & -- & .0000 & & & .000 & & & .0003 & \\
\hline Atom & AN & X & $\mathrm{Y}$ & Z & $\mathrm{X}$ & $\mathrm{Y}$ & Z & $\mathrm{x}$ & $\mathrm{Y}$ & Z \\
\hline 1 & 6 & .00 & -.03 & .00 & .00 & .00 & .00 & .00 & -.05 & .00 \\
\hline 2 & 1 & .00 & .41 & .00 & .00 & .00 & .00 & .00 & .58 & .0 \\
\hline 3 & 6 & .00 & .02 & .03 & .00 & .02 & .04 & .00 & .01 & .0 \\
\hline 4 & 1 & .00 & -.20 & -.35 & .00 & -.25 & -.43 & .00 & -.15 & -.2 \\
\hline 5 & 6 & .00 & .02 & -.03 & .00 & .02 & -.04 & .00 & -.01 & .0 \\
\hline 6 & 1 & .00 & -.20 & .35 & .00 & -.25 & .43 & .00 & .15 & -.2 \\
\hline 7 & 6 & .00 & -.03 & .00 & .00 & .00 & .00 & .00 & .05 & .0 \\
\hline 8 & 1 & .00 & .41 & .00 & .00 & .00 & .00 & .00 & -.57 & .0 \\
\hline 9 & 6 & .00 & .02 & .03 & .00 & -.02 & -.04 & .00 & -.01 & -.0 \\
\hline 10 & 1 & .00 & -.20 & -.35 & .00 & .25 & .43 & .00 & .15 & .2 \\
\hline
\end{tabular}




\begin{tabular}{|c|c|c|c|c|c|c|c|c|c|c|}
\hline 11 & 6 & .00 & .02 & -.03 & .00 & -.02 & .04 & .00 & .01 & -.02 \\
\hline 12 & 1 & .00 & -.20 & .35 & .00 & .25 & -.43 & .00 & -.14 & .25 \\
\hline 13 & 1 & .00 & .00 & .00 & .00 & .00 & .00 & .00 & .00 & .00 \\
\hline 14 & 6 & .00 & .00 & .00 & .00 & .00 & .00 & .00 & .00 & .00 \\
\hline 15 & 1 & .00 & .00 & .00 & .00 & .00 & .00 & .00 & .00 & .00 \\
\hline 16 & 1 & .00 & .00 & .00 & .00 & .00 & .00 & .00 & .00 & \\
\hline \multirow[t]{3}{*}{17} & 1 & .00 & .00 & .00 & .00 & .00 & .00 & .00 & .00 & .0 \\
\hline & & & 43 & & & 44 & & & 45 & \\
\hline & & & ?A & & & ?A & & & $A^{\prime}$ & \\
\hline \multicolumn{2}{|c|}{ Frequencies } & -- & 3221.9801 & & \multicolumn{3}{|c|}{3221.9986} & \multicolumn{3}{|c|}{3231.5110} \\
\hline \multicolumn{2}{|c|}{ Red. masses } & -- & 1.0964 & & \multicolumn{3}{|c|}{1.0964} & \multicolumn{3}{|c|}{1.1012} \\
\hline \multicolumn{2}{|c|}{ Frc consts } & -- & 6.7058 & & \multicolumn{3}{|c|}{6.7059} & \multicolumn{3}{|c|}{6.7755} \\
\hline \multicolumn{2}{|c|}{ IR Inten } & -- & 25.6879 & & \multicolumn{3}{|c|}{25.6165} & \multicolumn{3}{|c|}{.1081} \\
\hline Atom & AN & $\mathrm{x}$ & $\mathrm{Y}$ & $\mathrm{Z}$ & $\mathrm{X}$ & $\mathrm{Y}$ & Z & $\mathrm{x}$ & $\mathrm{Y}$ & Z \\
\hline 1 & 6 & .00 & .00 & .00 & .00 & -.05 & .00 & .00 & -.04 & . \\
\hline 2 & 1 & .00 & -.01 & .00 & .00 & .57 & .00 & .00 & .41 & .00 \\
\hline 3 & 6 & .00 & -.02 & -.04 & .00 & -.01 & -.02 & .00 & -.02 & -.0 \\
\hline 4 & 1 & .00 & .25 & .43 & .00 & .15 & .26 & .00 & .20 & .3 \\
\hline 5 & 6 & .00 & .02 & -.04 & .00 & -.01 & .02 & .00 & .02 & -.0 \\
\hline 6 & 1 & .00 & -.25 & .43 & .00 & .13 & -.24 & .00 & -.20 & .3 \\
\hline 7 & 6 & .00 & .00 & .00 & .00 & -.05 & .00 & .00 & .04 & .0 \\
\hline 8 & 1 & .00 & -.01 & .00 & .00 & .58 & .00 & .00 & -.41 & .0 \\
\hline 9 & 6 & .00 & -.02 & -.04 & .00 & -.01 & -.02 & .00 & .02 & .0 \\
\hline 10 & 1 & .00 & .25 & .42 & .00 & .15 & .26 & .00 & -.20 & -.3 \\
\hline 11 & 6 & .00 & .02 & -.04 & .00 & -.01 & .02 & .00 & -.02 & .0 \\
\hline 12 & 1 & .00 & -.26 & .44 & .00 & .13 & -.24 & .00 & .20 & -.3 \\
\hline 13 & 1 & .00 & .00 & .00 & .00 & .00 & .00 & .00 & .00 & .0 \\
\hline 14 & 6 & .00 & .00 & .00 & .00 & .00 & .00 & .00 & .00 & \\
\hline 15 & 1 & .00 & .00 & .00 & .00 & .00 & .00 & .00 & .00 & .0 \\
\hline 16 & 1 & .00 & .00 & .00 & .00 & .00 & .00 & .00 & .00 & .0 \\
\hline 17 & 1 & .00 & .00 & .00 & .00 & .00 & .00 & .00 & .00 & \\
\hline
\end{tabular}

- Thermochemistry -

Temperature 298.150 Kelvin. Pressure 1.00000 Atm.

Atom 1 has atomic number 6 and mass 12.00000

Atom 2 has atomic number 1 and mass 1.00783

Atom 3 has atomic number 6 and mass 12.00000

Atom 4 has atomic number 1 and mass 1.00783

Atom 5 has atomic number 6 and mass 12.00000

Atom 6 has atomic number 1 and mass 1.00783

Atom 7 has atomic number 6 and mass 12.00000

Atom 8 has atomic number 1 and mass 1.00783

Atom 9 has atomic number 6 and mass 12.00000

Atom 10 has atomic number 1 and mass 1.00783

Atom 11 has atomic number 6 and mass 12.00000

Atom 12 has atomic number 1 and mass 1.00783

Atom 13 has atomic number 1 and mass 2.01410

Atom 14 has atomic number 6 and mass 12.00000

Atom 15 has atomic number 1 and mass 2.01410

Atom 16 has atomic number 1 and mass 2.01410

Atom 17 has atomic number 1 and mass 1.00783

Molecular mass: 97.09708 amu.

Principal axes and moments of inertia in atomic units:

$\begin{array}{cccc} & 1 & 2 & 3 \\ \text { EIGENVALUES } & -- & 656.431521064 .446231068 .64963 \\ X & .99956 & .00000 & .02972\end{array}$




\begin{tabular}{|c|c|c|c|c|c|}
\hline-.01 & 486 & 6606 & .49972 & & \\
\hline-.02 & -.4 & 9994 & .86568 & & \\
\hline This molecule is an asymmet & fic top. & & & & \\
\hline Rotational symmetry number & & & & & \\
\hline $\begin{array}{r}\text { Warning -- assumption of } \mathrm{Cl} \\
\text { may cause signifi }\end{array}$ & $\begin{array}{l}\text { assical b } \\
\text { tant erro }\end{array}$ & $\begin{array}{l}\text { ehavior } \\
\text { r }\end{array}$ & for rotati & & \\
\hline Rotational temperatures (Ke & (vin) & .1319 & $5 \quad .08$ & 137 & .08105 \\
\hline Rotational constants (GHZ): & & 2.7493 & 1.69 & 547 & 1.68881 \\
\hline Zero-point vibrational ener & & 8203.5 & Joules/Mol & & \\
\hline & & .61270 & Kcal/Mol) & & \\
\hline Warning -- explicit conside & cation of & 11 deg & rees of fr & eedom as & \\
\hline Vibrational temperatures: & 27.26 & 55.95 & 57.71 & 112.68 & 122.63 \\
\hline (Kelvin) & 131.57 & 531.81 & 531.81 & 675.94 & 875.90 \\
\hline & 875.90 & 959.10 & 1200.42 & 1200.43 & 1291.27 \\
\hline & 1291.28 & 1334.69 & 1451.04 & 1454.85 & 1491.68 \\
\hline & 1523.73 & 1524.32 & 1526.21 & 1533.58 & 1688.41 \\
\hline & 1724.07 & 1724.08 & 1905.18 & 1909.62 & 1967.36 \\
\hline & 2087.60 & 2165.04 & 2165.05 & 2353.41 & 2353.42 \\
\hline & 3192.55 & 3409.64 & 3429.54 & 4562.82 & 4597.71 \\
\hline & 4612.86 & 4612.89 & 4635.71 & 4635.73 & 4649.42 \\
\hline Zero-point correction= & & & .13 & 433 (Ha & tree/Par \\
\hline
\end{tabular}


XXV. Harmonic frequency analysis and zero-point energy for 1,2,3,4-tetradeuterated methane-benzene complex with the MP2/6-311++G(d,p) method:

Full mass-weighted force constant matrix:

$\begin{array}{ccccc}\text { Low frequencies }--- & -6.1332 & -3.2555 & - \\ 3.1608 \quad .0038 & .0039 & .0040 & \\ \text { Low frequencies }--- & 17.2955 & 38.2020 & 38.2093 \\ \text { Diagonal vibrational polarizability: } & \end{array}$

Diagonal vibrational polarizability:

$$
8.0810770 \quad .8875698 \quad .8874588
$$

Harmonic frequencies ( $\left.\mathrm{cm}^{\star} \star-1\right)$, IR intensities (KM/Mole), Raman scattering activities (A**4/AMU), depolarization ratios for plane and unpolarized incident light, reduced masses (AMU), force constants (mDyne/A), and normal coordinates:

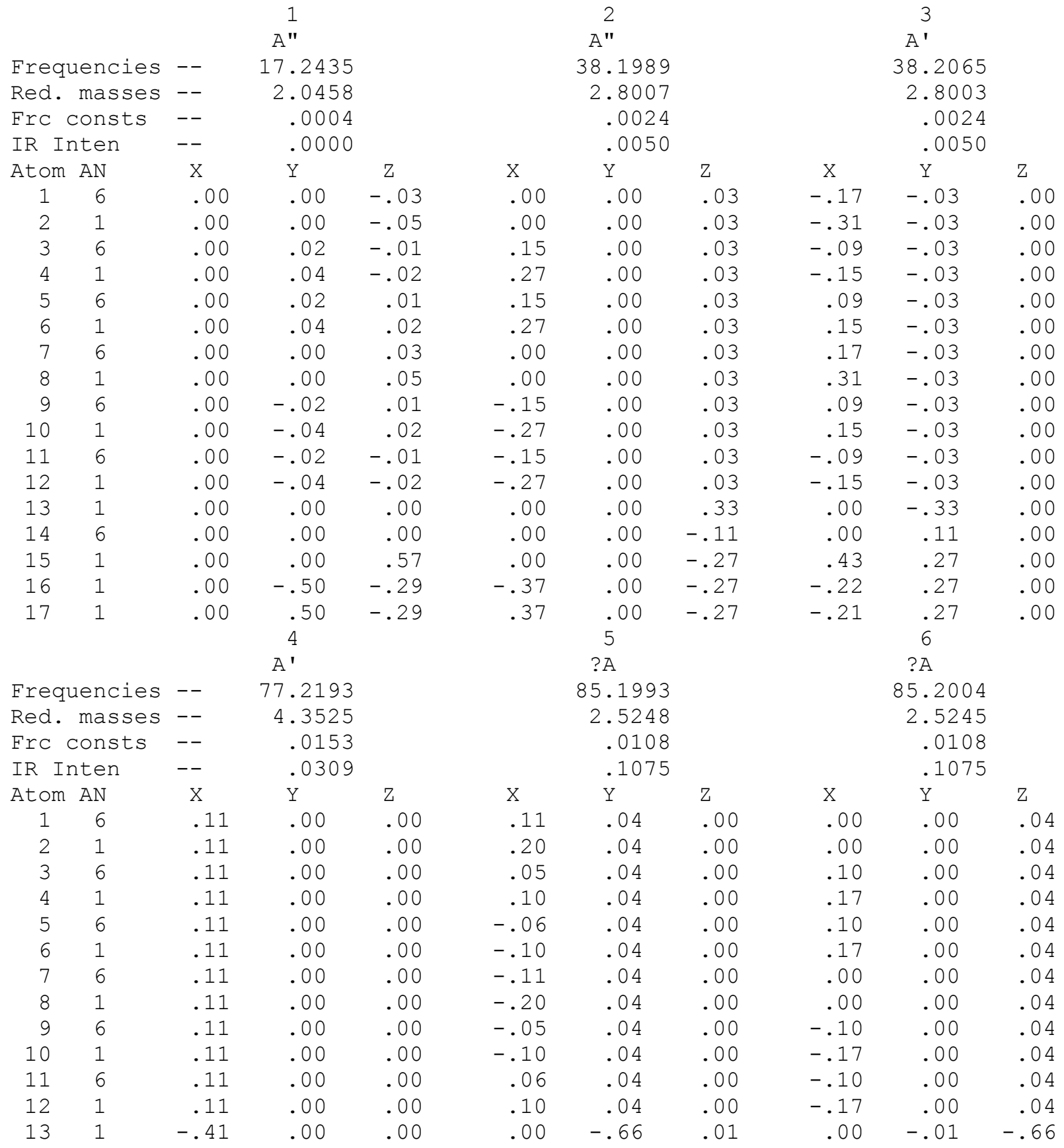




\begin{tabular}{|c|c|c|c|c|c|c|c|c|c|c|}
\hline 14 & 6 & -.42 & .00 & .00 & .00 & -.14 & .00 & .00 & .00 & -.14 \\
\hline 15 & 1 & -.42 & .00 & .00 & .50 & .04 & .00 & .00 & .00 & .04 \\
\hline 16 & 1 & -.42 & .00 & .00 & -.25 & .04 & .00 & .43 & .00 & .04 \\
\hline \multirow[t]{2}{*}{17} & 1 & -.42 & .00 & .00 & -.25 & $\dot{8}^{.04}$ & .00 & -.43 & $\dot{9}^{.00}$ & .04 \\
\hline & & & $A^{\prime}$ & & & $\mathrm{A}^{\prime \prime}$ & & \multicolumn{3}{|c|}{$A^{\prime}$} \\
\hline \multicolumn{2}{|c|}{ Frequencies } & -- & \multicolumn{2}{|l|}{369.6276} & \multicolumn{3}{|c|}{369.6288} & \multicolumn{3}{|c|}{469.8032} \\
\hline Red. & masses & -- & \multicolumn{2}{|l|}{3.4124} & \multicolumn{3}{|c|}{3.4124} & \multicolumn{3}{|c|}{9.9360} \\
\hline \multicolumn{2}{|c|}{ Frc consts } & -- & \multicolumn{2}{|l|}{.2747} & \multicolumn{3}{|c|}{.2747} & \multicolumn{3}{|c|}{1.2921} \\
\hline \multicolumn{2}{|c|}{ IR Inten } & -- & \multicolumn{2}{|l|}{.0004} & \multicolumn{3}{|c|}{.0004} & & .0000 & \\
\hline Atom & AN & $\mathrm{X}$ & Y & Z & $\mathrm{X}$ & $\mathrm{Y}$ & Z & $\mathrm{X}$ & $\mathrm{Y}$ & Z \\
\hline 1 & 6 & .27 & .00 & .00 & .00 & .00 & .00 & .37 & .00 & .00 \\
\hline 2 & 1 & .51 & .00 & .00 & .00 & .00 & .00 & .18 & .00 & .00 \\
\hline 3 & 6 & -.13 & .00 & .00 & .23 & .00 & .00 & -.37 & .00 & .00 \\
\hline 4 & 1 & -.26 & .00 & .00 & .44 & .00 & .00 & -.18 & .00 & .00 \\
\hline 5 & 6 & -.14 & .00 & .00 & -.23 & .00 & .00 & .37 & .00 & .00 \\
\hline 6 & 1 & -.25 & .00 & .00 & -.44 & .00 & .00 & .18 & .00 & .00 \\
\hline 7 & 6 & .27 & .00 & .00 & .00 & .00 & .00 & -.37 & .00 & .00 \\
\hline 8 & 1 & .51 & .00 & .00 & .00 & .00 & .00 & -.18 & .00 & .00 \\
\hline 9 & 6 & -.13 & .00 & .00 & .23 & .00 & .00 & .37 & .00 & .00 \\
\hline 10 & 1 & -.25 & .00 & .00 & .44 & .00 & .00 & .18 & .00 & .00 \\
\hline 11 & 6 & -.14 & .00 & .00 & -.23 & .00 & .00 & -.37 & .00 & .00 \\
\hline 12 & 1 & -.26 & .00 & .00 & -.44 & .00 & .00 & -.18 & .00 & .00 \\
\hline 13 & 1 & .00 & .00 & .00 & .00 & .00 & .00 & .00 & .00 & .00 \\
\hline 14 & 6 & .00 & .00 & .00 & .00 & .00 & .00 & .00 & .00 & .00 \\
\hline 15 & 1 & .00 & .00 & .00 & .00 & .00 & .00 & .00 & .00 & .00 \\
\hline 16 & 1 & .00 & .00 & .00 & .00 & .00 & .00 & .00 & .00 & .00 \\
\hline 17 & 1 & .00 & .00 & .00 & .00 & .00 & .00 & .00 & .00 & .00 \\
\hline & & & 10 & & & 11 & & & 12 & \\
\hline & & & ?A & & & ?A & & & $A^{\prime}$ & \\
\hline Frequ & dencies & -- & 608.7809 & & & 608.7815 & & & 666.6092 & \\
\hline Red. & masses & -- & 6.0129 & & & 6.0129 & & & 1.0850 & \\
\hline Frc $C$ & yonsts & -- & 1.3130 & & & 1.3130 & & & .2841 & \\
\hline IR In & iten & -- & .0000 & & & .0000 & & & 127.8922 & \\
\hline Atom & AN & $\mathrm{X}$ & Y & Z & $\mathrm{X}$ & Y & Z & $\mathrm{X}$ & $\mathrm{Y}$ & Z \\
\hline 1 & 6 & .00 & .00 & .15 & .00 & .36 & .00 & -.03 & .00 & .00 \\
\hline 2 & 1 & .00 & .00 & -.23 & -.01 & .36 & .00 & .41 & .00 & .00 \\
\hline 3 & 6 & .00 & .22 & .23 & .00 & .03 & -.22 & -.03 & .00 & .00 \\
\hline 4 & 1 & -.01 & .05 & .33 & .00 & -.26 & -.05 & .40 & .00 & .00 \\
\hline 5 & 6 & .00 & .22 & -.23 & .00 & -.03 & -.22 & -.03 & .00 & .00 \\
\hline 6 & 1 & .01 & .05 & -.33 & .00 & .26 & -.05 & .41 & .00 & .00 \\
\hline 7 & 6 & .00 & .00 & -.15 & .00 & -.36 & .00 & -.03 & .00 & .00 \\
\hline 8 & 1 & .00 & .00 & .23 & -.01 & -.36 & .00 & .40 & .00 & .00 \\
\hline 9 & 6 & .00 & -.22 & -.23 & .00 & -.03 & .22 & -.03 & .00 & .00 \\
\hline 10 & 1 & -.01 & -.05 & -.33 & .00 & .26 & .05 & .41 & .00 & .00 \\
\hline 11 & 6 & .00 & -.22 & .23 & .00 & .03 & .22 & -.03 & .00 & .00 \\
\hline 12 & 1 & .01 & -.05 & .33 & .00 & -.26 & .05 & .40 & .00 & .00 \\
\hline 13 & 1 & .00 & .00 & .00 & .00 & .00 & .00 & .00 & .00 & .00 \\
\hline 14 & 6 & .00 & .00 & .00 & .00 & .00 & .00 & .00 & .00 & .00 \\
\hline 15 & 1 & .00 & .00 & .00 & .00 & .00 & .00 & .00 & .00 & .00 \\
\hline 16 & 1 & .00 & .00 & .00 & .00 & .00 & .00 & .00 & .00 & .00 \\
\hline 17 & 1 & .00 & .00 & .00 & .00 & .00 & .00 & .00 & .00 & .00 \\
\hline & & & 13 & & & 14 & & & 15 & \\
\hline & & & $A^{\prime}$ & & & $\mathrm{A}^{\prime \prime}$ & & & $A^{\prime}$ & \\
\hline Frequ & dencies & -- & 834.3340 & & & 834.3429 & & & 897.4790 & \\
\hline Red. & masses & -- & 1.2494 & & & 1.2494 & & & 1.2780 & \\
\hline Frc $C$ & yonsts & -- & .5124 & & & .5124 & & & .6065 & \\
\hline
\end{tabular}




\begin{tabular}{|c|c|c|c|c|c|c|c|c|c|c|}
\hline IR I & nten & -- & .134 & & & .134 & & & .0015 & \\
\hline Atom & AN & $\mathrm{x}$ & $\mathrm{Y}$ & Z & $\mathrm{X}$ & $\mathrm{Y}$ & Z & $\mathrm{x}$ & $\mathrm{Y}$ & Z \\
\hline 1 & 6 & -.09 & .00 & .00 & .00 & .00 & .00 & -.09 & .00 & .00 \\
\hline 2 & 1 & .60 & .00 & .00 & .00 & .00 & .00 & .54 & .00 & .00 \\
\hline 3 & 6 & -.04 & .00 & .00 & .07 & .00 & .00 & .05 & .00 & .00 \\
\hline 4 & 1 & .27 & .00 & .00 & -.47 & .00 & .00 & -.30 & .00 & .00 \\
\hline 5 & 6 & .04 & .00 & .00 & .08 & .00 & .00 & .04 & .00 & .00 \\
\hline 6 & 1 & -.30 & .00 & .00 & -.52 & .00 & .00 & -.27 & .00 & .00 \\
\hline 7 & 6 & .08 & .00 & .00 & .00 & .00 & .00 & -.09 & .00 & .00 \\
\hline 8 & 1 & -.54 & .00 & .00 & .00 & .00 & .00 & .60 & .00 & .00 \\
\hline 9 & 6 & .04 & .00 & .00 & -.08 & .00 & .00 & .04 & .00 & .00 \\
\hline 10 & 1 & -.30 & .00 & .00 & .52 & .00 & .00 & -.27 & .00 & .00 \\
\hline 11 & 6 & -.04 & .00 & .00 & -.07 & .00 & .00 & .05 & .00 & .00 \\
\hline 12 & 1 & .27 & .00 & .00 & .47 & .00 & .00 & -.30 & .00 & .00 \\
\hline 13 & 1 & .00 & .01 & .00 & .00 & .00 & -.01 & .00 & .00 & .00 \\
\hline 14 & 6 & .00 & .00 & .00 & .00 & .00 & .00 & .00 & .00 & .00 \\
\hline 15 & 1 & .00 & .00 & .00 & .00 & .00 & .00 & .00 & .00 & .00 \\
\hline 16 & 1 & .00 & .00 & .00 & .00 & .00 & .00 & .00 & .00 & .00 \\
\hline 17 & 1 & .00 & .00 & .00 & .00 & .00 & .00 & .00 & .00 & .00 \\
\hline & & & 16 & & & 17 & & & 18 & \\
\hline & & & A" & & & $A^{\prime}$ & & & $A^{\prime}$ & \\
\hline Freq & uencies & -- & 897.487 & & & .7 .656 & & & 008.4913 & \\
\hline Red. & masses & -- & 1.278 & & & 1.025 & & & 5.9451 & \\
\hline Frc & consts & -- & .606 & & & .520 & & & 3.5625 & \\
\hline IR I & nten & -- & .001 & & & .003 & & & .0026 & \\
\hline Atom & AN & $\mathrm{x}$ & $\mathrm{Y}$ & Z & $\mathrm{x}$ & $\mathrm{Y}$ & Z & $\mathrm{x}$ & $\mathrm{Y}$ & Z \\
\hline 1 & 6 & .00 & .00 & .00 & .02 & .00 & .00 & .00 & .27 & .00 \\
\hline 2 & 1 & .00 & .00 & .00 & -.41 & .00 & .00 & .00 & .30 & .00 \\
\hline 3 & 6 & -.08 & .00 & .00 & -.02 & .00 & .00 & .00 & .14 & .24 \\
\hline 4 & 1 & .52 & .00 & .00 & .41 & .00 & .00 & .00 & .15 & .26 \\
\hline 5 & 6 & .07 & .00 & .00 & .02 & .00 & .00 & .00 & -.14 & .23 \\
\hline 6 & 1 & -.47 & .00 & .00 & -.41 & .00 & .00 & .00 & -.15 & .26 \\
\hline 7 & 6 & .00 & .00 & .00 & -.02 & .00 & .00 & .00 & -.28 & .00 \\
\hline 8 & 1 & .00 & .00 & .00 & .41 & .00 & .00 & .00 & -.30 & .00 \\
\hline 9 & 6 & -.07 & .00 & .00 & .02 & .00 & .00 & .00 & -.14 & -.23 \\
\hline 10 & 1 & .47 & .00 & .00 & -.41 & .00 & .00 & .00 & -.15 & -.26 \\
\hline 11 & 6 & .08 & .00 & .00 & -.02 & .00 & .00 & .00 & .14 & -.24 \\
\hline 12 & 1 & -.52 & .00 & .00 & .41 & .00 & .00 & .00 & .15 & -.26 \\
\hline 13 & 1 & .00 & .00 & .00 & .00 & .00 & .00 & .02 & .00 & .00 \\
\hline 14 & 6 & .00 & .00 & .00 & .00 & .00 & .00 & .02 & .00 & .00 \\
\hline 15 & 1 & .00 & .00 & .00 & .00 & .00 & .00 & -.04 & -.02 & .00 \\
\hline 16 & 1 & .00 & .00 & .00 & .00 & .00 & .00 & -.04 & .01 & -.02 \\
\hline 17 & 1 & .00 & .00 & .00 & .00 & .00 & .00 & -.04 & .01 & .02 \\
\hline & & & 19 & & & 20 & & & 21 & \\
\hline & & & $A^{\prime}$ & & & $A^{\prime}$ & & & A" & \\
\hline Freq & uencies & -- & 1011.172 & & & 3.283 & & & 029.0708 & \\
\hline Red. & masses & -- & 6.536 & & & 2.475 & & & 2.4678 & \\
\hline Frc & consts & -- & 3.937 & & & 1.527 & & & 1.5397 & \\
\hline IR I & nten & -- & .000 & & & $6.71 \varepsilon$ & & & 2.2979 & \\
\hline Atom & AN & $\mathrm{x}$ & Y & Z & $\mathrm{X}$ & $\mathrm{Y}$ & Z & $\mathrm{X}$ & Y & Z \\
\hline 1 & 6 & .00 & .29 & .00 & .00 & -.01 & .00 & .00 & .00 & .00 \\
\hline 2 & 1 & .00 & .29 & .00 & .00 & -.01 & .00 & .00 & .00 & -.03 \\
\hline 3 & 6 & .00 & -.14 & -.25 & .00 & .00 & -.01 & .00 & .00 & .00 \\
\hline 4 & 1 & .00 & -.14 & -.25 & .00 & -.01 & -.01 & -.01 & .01 & .00 \\
\hline 5 & 6 & .00 & -.15 & .25 & .00 & .00 & -.01 & .00 & .00 & .00 \\
\hline 6 & 1 & .00 & -.15 & .25 & .00 & .01 & -.01 & .00 & -.01 & .00 \\
\hline 7 & 6 & .00 & .29 & .00 & .00 & .01 & .00 & .00 & .00 & .00 \\
\hline
\end{tabular}




\begin{tabular}{|c|c|c|c|c|c|c|c|c|c|c|}
\hline 8 & 1 & .00 & .29 & .00 & .00 & .01 & .00 & .00 & .00 & -.02 \\
\hline 9 & 6 & .00 & -.15 & -.25 & .00 & .00 & .01 & .00 & .00 & .00 \\
\hline 10 & 1 & .00 & -.15 & -.25 & .00 & .01 & .01 & .00 & .01 & .00 \\
\hline 11 & 6 & .00 & -.14 & .25 & .00 & .00 & .01 & .00 & .00 & .00 \\
\hline 12 & 1 & .00 & -.14 & .25 & .00 & -.01 & .01 & .01 & -.01 & .00 \\
\hline 13 & 1 & .00 & .00 & .00 & .21 & .00 & .00 & .00 & .00 & .58 \\
\hline 14 & 6 & .00 & .00 & .00 & .21 & .00 & .00 & .00 & .00 & -.21 \\
\hline 15 & 1 & .00 & .00 & .00 & -.49 & -.25 & .00 & .00 & .00 & .59 \\
\hline 16 & 1 & .00 & .00 & .00 & -.49 & .12 & -.21 & .20 & .31 & .06 \\
\hline \multirow{3}{*}{17} & 1 & .00 & .00 & .00 & -.49 & .12 & .21 & -.20 & -.31 & .06 \\
\hline & & & 22 & & & 23 & & & 24 & \\
\hline & & & $A^{\prime}$ & & & A" & & & $A^{\prime}$ & \\
\hline \multicolumn{2}{|c|}{ Frequencies } & -- & \multicolumn{2}{|c|}{1029.0711} & \multicolumn{3}{|c|}{1059.4806} & \multicolumn{3}{|c|}{1059.4855} \\
\hline \multicolumn{2}{|c|}{ Red. masses } & -- & 2.4678 & & \multicolumn{3}{|c|}{1.6992} & \multicolumn{3}{|c|}{1.6992} \\
\hline \multicolumn{2}{|c|}{ Frc consts } & -- & 1.5397 & & \multicolumn{3}{|c|}{1.1238} & \multicolumn{3}{|c|}{1.1238} \\
\hline \multicolumn{2}{|c|}{ IR Inten } & -- & 2.2979 & & \multicolumn{3}{|c|}{4.9163} & \multicolumn{3}{|c|}{4.9163} \\
\hline Atom & AN & $\mathrm{x}$ & $\mathrm{Y}$ & Z & $\mathrm{x}$ & $\mathrm{Y}$ & $\mathrm{Z}$ & $\mathrm{x}$ & $\mathrm{Y}$ & $\mathrm{Z}$ \\
\hline 1 & 6 & .00 & .01 & .00 & .00 & .00 & .08 & .00 & -.12 & .00 \\
\hline 2 & 1 & .00 & .01 & .00 & .00 & .00 & .54 & .00 & -.13 & .00 \\
\hline 3 & 6 & .00 & .00 & .00 & .00 & -.09 & -.07 & .00 & .03 & -.09 \\
\hline 4 & 1 & .00 & -.01 & .01 & .00 & -.29 & .04 & .00 & .38 & -.29 \\
\hline 5 & 6 & .00 & .00 & .00 & .00 & .09 & -.07 & .00 & .03 & .09 \\
\hline 6 & 1 & .00 & -.02 & -.01 & .00 & .29 & .04 & .00 & .38 & .29 \\
\hline 7 & 6 & .00 & .01 & .00 & .00 & .00 & .08 & .00 & -.12 & .00 \\
\hline 8 & 1 & .01 & .01 & .00 & .00 & .00 & .54 & .00 & -.13 & .00 \\
\hline 9 & 6 & .00 & .00 & .00 & .00 & -.09 & -.07 & .00 & .03 & -.09 \\
\hline 10 & 1 & .00 & -.02 & .01 & .00 & -.29 & .04 & .00 & .38 & -.29 \\
\hline 11 & 6 & .00 & .00 & .00 & .00 & .09 & -.07 & .00 & .03 & .09 \\
\hline 12 & 1 & .00 & -.01 & -.01 & .00 & .29 & .04 & .00 & .38 & .29 \\
\hline 13 & 1 & .00 & .58 & .00 & .00 & .00 & .01 & .00 & .01 & .00 \\
\hline 14 & 6 & .00 & -.21 & .00 & .00 & .00 & -.01 & .00 & -.01 & .00 \\
\hline 15 & 1 & .23 & -.12 & .00 & .00 & .00 & .03 & .00 & -.01 & .00 \\
\hline 16 & 1 & -.12 & .41 & .31 & .00 & .02 & .00 & .00 & .02 & .02 \\
\hline \multirow[t]{3}{*}{17} & 1 & -.12 & .41 & -.31 & .00 & -.02 & .00 & .00 & .02 & -.02 \\
\hline & & & 25 & & & 26 & & & 27 & \\
\hline & & & $A^{\prime}$ & & & A" & & & $\mathrm{A}^{\prime \prime}$ & \\
\hline Frequ & lencies & -- & 1113.2847 & & & 13.284 & & & 173.5072 & \\
\hline Red. & masses & -- & 2.0137 & & & 2.013 & & & 1.0313 & \\
\hline Frc & onsts & -- & 1.4705 & & & 1.470 & & & .8368 & \\
\hline IR In & ten & -- & 1.0073 & & & 1.007 & & & .0000 & \\
\hline Atom & AN & $\mathrm{x}$ & $\mathrm{Y}$ & Z & $\mathrm{x}$ & $\mathrm{Y}$ & Z & $\mathrm{x}$ & $\mathrm{Y}$ & Z \\
\hline 1 & 6 & .00 & .00 & .00 & .00 & .00 & .00 & .00 & .00 & .02 \\
\hline 2 & 1 & .00 & .00 & .00 & .00 & .00 & .02 & .00 & .00 & .41 \\
\hline 3 & 6 & .00 & .00 & .00 & .00 & .00 & .00 & .00 & .02 & -.01 \\
\hline 4 & 1 & .00 & .01 & -.01 & .00 & -.01 & .00 & .00 & .35 & -.20 \\
\hline 5 & 6 & .00 & .00 & .00 & .00 & .00 & .00 & .00 & -.02 & -.01 \\
\hline 6 & 1 & .00 & .01 & .01 & .00 & .01 & .00 & .00 & -.35 & -.20 \\
\hline 7 & 6 & .00 & .00 & .00 & .00 & .00 & .00 & .00 & .00 & .02 \\
\hline 8 & 1 & .00 & .00 & .00 & .00 & .00 & .01 & .00 & .00 & .41 \\
\hline 9 & 6 & .00 & .00 & .00 & .00 & .00 & .00 & .00 & .02 & -.01 \\
\hline 10 & 1 & .00 & .01 & -.01 & .00 & -.01 & .00 & .00 & .35 & -.20 \\
\hline 11 & 6 & .00 & .00 & .00 & .00 & .00 & .00 & .00 & -.02 & -.01 \\
\hline 12 & 1 & .00 & .01 & .01 & .00 & .01 & .00 & .00 & -.35 & -.20 \\
\hline 13 & 1 & .00 & .51 & .00 & .00 & .00 & .51 & .00 & .00 & .00 \\
\hline 14 & 6 & .00 & .00 & .00 & .00 & .00 & .00 & .00 & .00 & .00 \\
\hline 15 & 1 & .47 & .16 & .00 & .00 & .00 & -.49 & .00 & .00 & .00 \\
\hline 16 & 1 & -.24 & -.33 & -.28 & .41 & -.29 & .00 & .00 & .00 & .00 \\
\hline
\end{tabular}




\begin{tabular}{|c|c|c|c|c|c|c|c|c|c|c|}
\hline 17 & 1 & -.23 & $\begin{array}{l}-.33 \\
28 \\
\text { A" }\end{array}$ & .28 & -.41 & $\begin{array}{l}2 \dot{9}^{28} \\
\mathrm{~A}^{\prime}\end{array}$ & .00 & .00 & $\begin{array}{l}.00 \\
30 \\
\text { A" }\end{array}$ & .00 \\
\hline \multicolumn{2}{|c|}{ Frequencies } & --1 & & 1198.2940 & \multicolumn{3}{|c|}{1198.2973} & \multicolumn{3}{|c|}{1367.3845} \\
\hline \multicolumn{2}{|c|}{ Red. masses } & -- & \multirow{2}{*}{\multicolumn{2}{|c|}{$\begin{array}{r}1.1288 \\
.9550\end{array}$}} & \multicolumn{3}{|c|}{1.1288} & \multicolumn{3}{|c|}{1.2471} \\
\hline \multicolumn{2}{|c|}{ Frc consts } & -- & & & \multirow{2}{*}{\multicolumn{3}{|c|}{$\begin{array}{l}.9550 \\
.0002\end{array}$}} & \multicolumn{3}{|c|}{$\begin{array}{r}1.3738 \\
0000\end{array}$} \\
\hline \multicolumn{2}{|c|}{ IR Inten } & -- & \multicolumn{2}{|l|}{.0002} & & & & & .0000 & \\
\hline Atom & AN & X & $\mathrm{Y}$ & $\mathrm{Z}$ & $\mathrm{x}$ & Y & Z & $\mathrm{x}$ & $\mathrm{Y}$ & $\mathrm{Z}$ \\
\hline 1 & 6 & .00 & .00 & .06 & .00 & .01 & .00 & .00 & .00 & -.06 \\
\hline 2 & 1 & .00 & .00 & .57 & .00 & .00 & .00 & .00 & .00 & .40 \\
\hline 3 & 6 & .00 & .03 & -.01 & .00 & .04 & -.03 & .00 & .05 & -.03 \\
\hline 4 & 1 & .00 & .25 & -.14 & .00 & .43 & -.25 & .00 & -.35 & .20 \\
\hline 5 & 6 & .00 & .03 & .01 & .00 & -.04 & -.03 & .00 & .05 & .03 \\
\hline 6 & 1 & .00 & .25 & .14 & .00 & -.43 & -.25 & .00 & -.35 & -.20 \\
\hline 7 & 6 & .00 & .00 & -.06 & .00 & -.01 & .00 & .00 & .00 & .06 \\
\hline 8 & 1 & .00 & .00 & -.57 & .00 & .00 & .00 & .00 & .00 & -.40 \\
\hline 9 & 6 & .00 & -.03 & .01 & .00 & -.04 & .03 & .00 & -.05 & .03 \\
\hline 10 & 1 & .00 & -.25 & .14 & .00 & -.43 & .25 & .00 & .35 & -.20 \\
\hline 11 & 6 & .00 & -.03 & -.01 & .00 & .04 & .03 & .00 & -.05 & -.03 \\
\hline 12 & 1 & .00 & -.25 & -.14 & .00 & .43 & .25 & .00 & .35 & .20 \\
\hline 13 & 1 & .00 & .00 & .00 & .00 & .00 & .00 & .00 & .00 & .00 \\
\hline 14 & 6 & .00 & .00 & .00 & .00 & .00 & .00 & .00 & .00 & .00 \\
\hline 15 & 1 & .00 & .00 & .00 & .00 & .00 & .00 & .00 & .00 & .00 \\
\hline 16 & 1 & .00 & .00 & .00 & .00 & .00 & .00 & .00 & .00 & .00 \\
\hline 17 & 1 & .00 & .00 & .00 & .00 & .00 & .00 & .00 & .00 & .00 \\
\hline & & & 31 & & & 32 & & & 33 & \\
\hline & & & A" & & & A" & & & $A^{\prime}$ & \\
\hline Frequ & lencies & --1 & 1450.9534 & & & 04.7746 & & & 504.7791 & \\
\hline Red. & masses & -- & 9.4417 & & & 2.0147 & & & 2.0141 & \\
\hline Frc c & onsts & -- & 11.7114 & & & $2.687 C$ & & & 2.6871 & \\
\hline IR In & ten & -- & .0000 & & & 7.2691 & & & 7.2691 & \\
\hline Atom & AN & $\mathrm{X}$ & $\mathrm{Y}$ & Z & $\mathrm{x}$ & $\mathrm{Y}$ & Z & $\mathrm{x}$ & Y & Z \\
\hline 1 & 6 & .00 & .00 & .36 & .00 & .00 & .15 & .00 & .09 & .00 \\
\hline 2 & 1 & .00 & .00 & -.20 & .00 & .00 & -.54 & .00 & .12 & .00 \\
\hline 3 & 6 & .00 & .31 & -.18 & .00 & -.10 & -.03 & .00 & -.09 & .10 \\
\hline 4 & 1 & .00 & -.17 & .10 & .00 & .18 & -.22 & .00 & .43 & -.18 \\
\hline 5 & 6 & .00 & -.31 & -.18 & .00 & .10 & -.03 & .00 & -.09 & -.10 \\
\hline 6 & 1 & .00 & .17 & .10 & .00 & -.18 & -.22 & .00 & .43 & .18 \\
\hline 7 & 6 & .00 & .00 & .36 & .00 & .00 & .15 & .00 & .09 & .00 \\
\hline 8 & 1 & .00 & .00 & -.20 & .00 & .00 & -.54 & .00 & .12 & .00 \\
\hline 9 & 6 & .00 & .31 & -.18 & .00 & -.10 & -.03 & .00 & -.09 & .10 \\
\hline 10 & 1 & .00 & -.17 & .10 & .00 & .18 & -.22 & .00 & .43 & -.18 \\
\hline 11 & 6 & .00 & -.31 & -.18 & .00 & .10 & -.03 & .00 & -.09 & -.10 \\
\hline 12 & 1 & .00 & .17 & .10 & .00 & -.18 & -.22 & .00 & .43 & .18 \\
\hline 13 & 1 & .00 & .00 & .00 & .00 & .00 & .00 & .00 & .00 & .00 \\
\hline 14 & 6 & .00 & .00 & .00 & .00 & .00 & .00 & .00 & .00 & .00 \\
\hline 15 & 1 & .00 & .00 & .00 & .00 & .00 & .00 & .00 & .00 & .00 \\
\hline 16 & 1 & .00 & .00 & .00 & .00 & .00 & .00 & .00 & .00 & .00 \\
\hline 17 & 1 & .00 & .00 & .00 & .00 & .00 & .00 & .00 & .00 & .00 \\
\hline & & & 34 & & & 35 & & & 36 & \\
\hline & & & $A^{\prime}$ & & & A" & & & $A^{\prime}$ & \\
\hline Frequ & lencies & -- & 1635.7060 & & & $35.707 \varepsilon$ & & & 171.4946 & \\
\hline Red. & masses & -- & 5.5549 & & & $5.555 c$ & & & 2.0145 & \\
\hline Frc c & onsts & -- & 8.7566 & & & $8.756 \varepsilon$ & & & 5.5968 & \\
\hline IR In & ten & -- & .0000 & & & .0000 & & & 2.0484 & \\
\hline Atom & AN & $\mathrm{X}$ & Y & Z & $\mathrm{x}$ & Y & Z & $\mathrm{x}$ & Y & Z \\
\hline 1 & 6 & .00 & .15 & .00 & .00 & .00 & .34 & .00 & .00 & .00 \\
\hline
\end{tabular}




\begin{tabular}{|c|c|c|c|c|c|c|c|c|c|c|}
\hline 2 & 1 & .00 & .17 & .00 & .00 & .00 & -.41 & .00 & .00 & .00 \\
\hline 3 & 6 & .00 & -.29 & .08 & .00 & .08 & -.20 & .00 & .00 & .00 \\
\hline 4 & 1 & .00 & .26 & -.25 & .00 & -.25 & -.03 & .00 & .00 & .00 \\
\hline 5 & 6 & .00 & .29 & .08 & .00 & .08 & .20 & .00 & .00 & .00 \\
\hline 6 & 1 & .00 & -.26 & -.25 & .00 & -.25 & .03 & .00 & .00 & .00 \\
\hline 7 & 6 & .00 & -.15 & .00 & .00 & .00 & -.34 & .00 & .00 & .00 \\
\hline 8 & 1 & .00 & -.17 & .00 & .00 & .00 & .41 & .00 & .00 & .00 \\
\hline 9 & 6 & .00 & .29 & -.08 & .00 & -.08 & .20 & .00 & .00 & .00 \\
\hline 10 & 1 & .00 & -.26 & .25 & .00 & .25 & .03 & .00 & .00 & .00 \\
\hline 11 & 6 & .00 & -.29 & -.08 & .00 & -.08 & -.20 & .00 & .00 & .0 \\
\hline 12 & 1 & .00 & .26 & .25 & .00 & .25 & -.03 & .00 & .00 & .0 \\
\hline 13 & 1 & .00 & .00 & .00 & .00 & .00 & .00 & .47 & .00 & .0 \\
\hline 14 & 6 & .00 & .00 & .00 & .00 & .00 & .00 & .01 & .00 & .00 \\
\hline 15 & 1 & .00 & .00 & .00 & .00 & .00 & .00 & -.17 & .48 & .00 \\
\hline 16 & 1 & .00 & .00 & .00 & .00 & .00 & .00 & -.17 & -.24 & .4 \\
\hline \multirow[t]{3}{*}{17} & 1 & .00 & .00 & .00 & .00 & .00 & .00 & -.17 & -.24 & -.4 \\
\hline & & & 37 & & & 38 & & & 39 & \\
\hline & & & A" & & & $A^{\prime}$ & & & $A^{\prime}$ & \\
\hline \multicolumn{2}{|c|}{ Frequencies } & -- & \multicolumn{2}{|l|}{2369.7958} & \multicolumn{3}{|c|}{2369.7983} & \multicolumn{3}{|c|}{2385.1460} \\
\hline Red. & masses & -- & 2.3677 & & \multicolumn{3}{|c|}{2.3677} & \multicolumn{3}{|c|}{2.3658} \\
\hline \multicolumn{2}{|c|}{ Frc consts } & -- & 7.8341 & & \multicolumn{3}{|c|}{7.8342} & \multicolumn{3}{|c|}{7.9297} \\
\hline & -- & 8.3941 & & \multicolumn{3}{|c|}{8.3941} & \multicolumn{3}{|c|}{2.4460} \\
\hline \multicolumn{2}{|c|}{$\begin{array}{l}\text { IR Inten } \\
\text { Atom AN }\end{array}$} & $\mathrm{X}$ & $\mathrm{Y}$ & Z & $\mathrm{x}$ & $\mathrm{Y}$ & Z & $\mathrm{x}$ & $\mathrm{Y}$ & Z \\
\hline 1 & 6 & .00 & .00 & .00 & .00 & .00 & .00 & .00 & .00 & .00 \\
\hline 2 & 1 & .00 & .00 & .00 & .00 & .00 & .00 & .00 & .00 & .0 \\
\hline 3 & 6 & .00 & .00 & .00 & .00 & .00 & .00 & .00 & .00 & .00 \\
\hline 4 & 1 & .00 & .00 & .00 & .00 & .00 & .00 & .00 & .00 & .0 \\
\hline 5 & 6 & .00 & .00 & .00 & .00 & .00 & .00 & .00 & .00 & .0 \\
\hline 6 & 1 & .00 & .00 & .00 & .00 & .00 & .00 & .00 & .00 & .0 \\
\hline 7 & 6 & .00 & .00 & .00 & .00 & .00 & .00 & .00 & .00 & .00 \\
\hline 8 & 1 & .00 & .00 & .00 & .00 & .00 & .00 & .00 & .00 & .0 \\
\hline 9 & 6 & .00 & .00 & .00 & .00 & .00 & .00 & .00 & .00 & .00 \\
\hline 10 & 1 & .00 & .00 & .00 & .00 & .00 & .00 & .00 & .00 & .0 \\
\hline 11 & 6 & .00 & .00 & .00 & .00 & .00 & .00 & .00 & .00 & .0 \\
\hline 12 & 1 & .00 & .00 & .00 & .00 & .00 & .00 & .00 & .00 & .0 \\
\hline 13 & 1 & .00 & .00 & .01 & .00 & .01 & .00 & .87 & .00 & .00 \\
\hline 14 & 6 & .00 & .00 & .19 & .00 & .19 & .00 & -.19 & .00 & .00 \\
\hline 15 & 1 & .00 & .00 & .00 & .27 & -.76 & .00 & .09 & -.25 & .0 \\
\hline 16 & 1 & .23 & .33 & -.57 & -.13 & -.19 & .33 & .09 & .12 & -.2 \\
\hline \multirow[t]{3}{*}{17} & 1 & -.23 & -.33 & -.57 & -.13 & -.19 & -.33 & .09 & .12 & .2 \\
\hline & & & 40 & & & 41 & & & 42 & \\
\hline & & & $A^{\prime}$ & & & $\mathrm{A}^{\prime \prime}$ & & & $A^{\prime}$ & \\
\hline Frequ & lencies & -- & 3195.5720 & & & 06.103 & & & 206.1225 & \\
\hline Red. & masses & -- & 1.0839 & & & 1.088 & & & 1.0883 & \\
\hline Frc & onsts & -- & 6.5215 & & & 6.590 & & & 6.5909 & \\
\hline IR Ir & ten & -- & .0000 & & & .000 & & & .0003 & \\
\hline Atom & AN & X & $\mathrm{Y}$ & Z & $\mathrm{X}$ & $\mathrm{Y}$ & Z & $\mathrm{x}$ & $\mathrm{Y}$ & Z \\
\hline 1 & 6 & .00 & -.03 & .00 & .00 & .00 & .00 & .00 & -.05 & .00 \\
\hline 2 & 1 & .00 & .41 & .00 & .00 & .00 & .00 & .00 & .58 & .0 \\
\hline 3 & 6 & .00 & .02 & .03 & .00 & .02 & .04 & .00 & .01 & .0 \\
\hline 4 & 1 & .00 & -.20 & -.35 & .00 & -.25 & -.43 & .00 & -.15 & -.2 \\
\hline 5 & 6 & .00 & .02 & -.03 & .00 & .02 & -.04 & .00 & -.01 & .0 \\
\hline 6 & 1 & .00 & -.20 & .35 & .00 & -.25 & .43 & .00 & .15 & -.2 \\
\hline 7 & 6 & .00 & -.03 & .00 & .00 & .00 & .00 & .00 & .05 & .0 \\
\hline 8 & 1 & .00 & .41 & .00 & .00 & .00 & .00 & .00 & -.57 & .0 \\
\hline 9 & 6 & .00 & .02 & .03 & .00 & -.02 & -.04 & .00 & -.01 & -.0 \\
\hline 10 & 1 & .00 & -.20 & -.35 & .00 & .25 & .43 & .00 & .15 & .2 \\
\hline
\end{tabular}




\begin{tabular}{|c|c|c|c|c|c|c|c|c|c|c|}
\hline 11 & 6 & .00 & .02 & -.03 & .00 & -.02 & .04 & .00 & .01 & -.02 \\
\hline 12 & 1 & .00 & -.20 & .35 & .00 & .25 & -.43 & .00 & -.15 & .25 \\
\hline 13 & 1 & .00 & .00 & .00 & .00 & .00 & .00 & .00 & .00 & .00 \\
\hline 14 & 6 & .00 & .00 & .00 & .00 & .00 & .00 & .00 & .00 & .00 \\
\hline 15 & 1 & .00 & .00 & .00 & .00 & .00 & .00 & .00 & .00 & .00 \\
\hline 16 & 1 & .00 & .00 & .00 & .00 & .00 & .00 & .00 & .00 & \\
\hline \multirow[t]{3}{*}{17} & 1 & .00 & .00 & .00 & .00 & .00 & .00 & .00 & .00 & .0 \\
\hline & & & 43 & & & 44 & & & 45 & \\
\hline & & & A" & & & $A^{\prime}$ & & & $A^{\prime}$ & \\
\hline \multicolumn{2}{|c|}{ Frequencies } & -- & 3221.9793 & & \multicolumn{3}{|c|}{3221.9983} & \multicolumn{3}{|c|}{3231.5107} \\
\hline \multicolumn{2}{|c|}{ Red. masses } & -- & 1.0964 & & \multicolumn{3}{|c|}{1.0964} & \multicolumn{3}{|c|}{1.1012} \\
\hline \multicolumn{2}{|c|}{ Frc consts } & -- & 6.7058 & & \multicolumn{3}{|c|}{6.7059} & \multicolumn{3}{|c|}{6.7755} \\
\hline \multicolumn{2}{|c|}{ IR Inten } & -- & 25.5752 & & \multicolumn{3}{|c|}{25.5745} & \multicolumn{3}{|c|}{.1100} \\
\hline Atom & AN & $\mathrm{x}$ & $\mathrm{Y}$ & $\mathrm{Z}$ & $\mathrm{X}$ & $\mathrm{Y}$ & Z & $\mathrm{x}$ & $\mathrm{Y}$ & Z \\
\hline 1 & 6 & .00 & .00 & .00 & .00 & -.05 & .00 & .00 & -.04 & $\cdot$ \\
\hline 2 & 1 & .00 & .00 & .00 & .00 & .57 & .00 & .00 & .41 & .00 \\
\hline 3 & 6 & .00 & -.02 & -.04 & .00 & -.01 & -.02 & .00 & -.02 & -.0 \\
\hline 4 & 1 & .00 & .25 & .43 & .00 & .14 & .25 & .00 & .20 & .3 \\
\hline 5 & 6 & .00 & .02 & -.04 & .00 & -.01 & .02 & .00 & .02 & -.0 \\
\hline 6 & 1 & .00 & -.25 & .43 & .00 & .14 & -.25 & .00 & -.20 & .3 \\
\hline 7 & 6 & .00 & .00 & .00 & .00 & -.05 & .00 & .00 & .04 & .0 \\
\hline 8 & 1 & .00 & .00 & .00 & .00 & .58 & .00 & .00 & -.41 & .0 \\
\hline 9 & 6 & .00 & -.02 & -.04 & .00 & -.01 & -.02 & .00 & .02 & .0 \\
\hline 10 & 1 & .00 & .25 & .43 & .00 & .14 & .25 & .00 & -.20 & -.3 \\
\hline 11 & 6 & .00 & .02 & -.04 & .00 & -.01 & .02 & .00 & -.02 & .0 \\
\hline 12 & 1 & .00 & -.25 & .43 & .00 & .14 & -.25 & .00 & .20 & -.3 \\
\hline 13 & 1 & .00 & .00 & .00 & .00 & .00 & .00 & .00 & .00 & .0 \\
\hline 14 & 6 & .00 & .00 & .00 & .00 & .00 & .00 & .00 & .00 & \\
\hline 15 & 1 & .00 & .00 & .00 & .00 & .00 & .00 & .00 & .00 & .0 \\
\hline 16 & 1 & .00 & .00 & .00 & .00 & .00 & .00 & .00 & .00 & .0 \\
\hline 17 & 1 & .00 & .00 & .00 & .00 & .00 & .00 & .00 & .00 & \\
\hline
\end{tabular}

- Thermochemistry -

Temperature 298.150 Kelvin. Pressure 1.00000 Atm.

Atom 1 has atomic number 6 and mass 12.00000

Atom 2 has atomic number 1 and mass 1.00783

Atom 3 has atomic number 6 and mass 12.00000

Atom 4 has atomic number 1 and mass 1.00783

Atom 5 has atomic number 6 and mass 12.00000

Atom 6 has atomic number 1 and mass 1.00783

Atom 7 has atomic number 6 and mass 12.00000

Atom 8 has atomic number 1 and mass 1.00783

Atom 9 has atomic number 6 and mass 12.00000

Atom 10 has atomic number 1 and mass 1.00783

Atom 11 has atomic number 6 and mass 12.00000

Atom 12 has atomic number 1 and mass 1.00783

Atom 13 has atomic number 1 and mass 2.01410

Atom 14 has atomic number 6 and mass 12.00000

Atom 15 has atomic number 1 and mass 2.01410

Atom 16 has atomic number 1 and mass 2.01410

Atom 17 has atomic number 1 and mass 2.01410

Molecular mass: 98.10336 amu.

Principal axes and moments of inertia in atomic units:

$$
\begin{array}{cccc}
\text { EIGENVALUES -- } & 660.634581107 .360961107 .36166 \\
\text { X } & 1.00000 & .00000 & .00000
\end{array}
$$




\begin{tabular}{|c|c|c|c|c|c|}
\hline \multicolumn{6}{|c|}{$.00000 \quad 1.00000 \quad .00000$} \\
\hline \multicolumn{6}{|c|}{$.00000 \quad .00000 \quad 1.00000$} \\
\hline \multirow{2}{*}{\multicolumn{6}{|c|}{$\begin{array}{l}\text { This molecule is an asymmetric top. } \\
\text { Rotational symmetry number } 1 \text {. }\end{array}$}} \\
\hline & & & & & \\
\hline \multicolumn{6}{|c|}{$\begin{array}{c}\text { Warning -- assumption of classical behavior for rotation } \\
\text { may cause significant error }\end{array}$} \\
\hline \multicolumn{2}{|c|}{ Rotational temperatures (Kelvin) } & .13111 & \multicolumn{2}{|c|}{.07822} & .07822 \\
\hline & 2.7318 & \multicolumn{2}{|c|}{1.62977} & 1.62977 \\
\hline \multicolumn{2}{|c|}{ Zero-point vibrational energy } & $.035 .4 \quad(J$ & $\begin{array}{l}\text { oules/Mol } \\
\text { cal/Mol) }\end{array}$ & & \\
\hline \multicolumn{6}{|c|}{$\begin{array}{c}\text { Warning -- explicit consideration of } 11 \text { degrees of freedom as } \\
\text { vibrations may cause significant error }\end{array}$} \\
\hline Vibrational temperatures: & 24.81 & 54.96 & 54.97 & 111.10 & 122.58 \\
\hline$($ Kelvin $)$ & 122.58 & 531.81 & 531.81 & 675.94 & 875.90 \\
\hline & 875.90 & 959.10 & 1200.42 & 1200.43 & 1291.27 \\
\hline & 1291.28 & 1334.69 & 1450.99 & 1454.85 & 1472.28 \\
\hline & 1480.60 & 1480.60 & 1524.35 & 1524.36 & 1601.77 \\
\hline & 1601.77 & 1688.41 & 1724.08 & 1724.08 & 1967.36 \\
\hline & 2087.60 & 2165.03 & 2165.04 & 2353.41 & 2353.42 \\
\hline & 3124.29 & 3409.60 & 3409.61 & 3431.69 & 4597.71 \\
\hline & 4612.86 & 4612.89 & 4635.70 & 4635.73 & 4649.42 \\
\hline
\end{tabular}


XXVI. Harmonic frequency analysis and zero-point energy for non-deuterated methane-tetrachloromethane complex with the MP2/6-311++G(d,p) method:

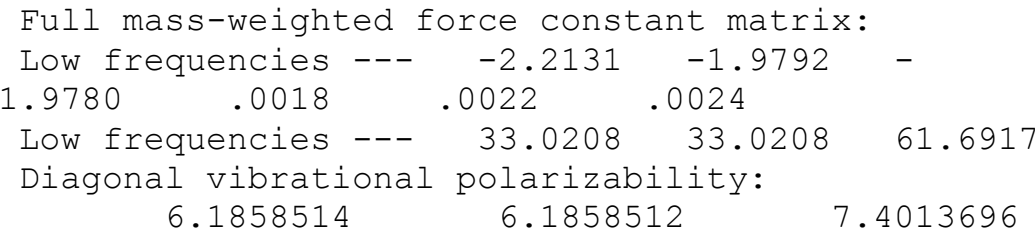

Harmonic frequencies $(\mathrm{cm} \star \star-1)$, IR intensities (KM/Mole), Raman scattering activities ( $A^{* * 4 / A M U), ~ d e p o l a r i z a t i o n ~ r a t i o s ~ f o r ~ p l a n e ~ a n d ~ u n p o l a r i z e d ~}$ incident light, reduced masses (AMU), force constants (mDyne/A), and normal coordinates:

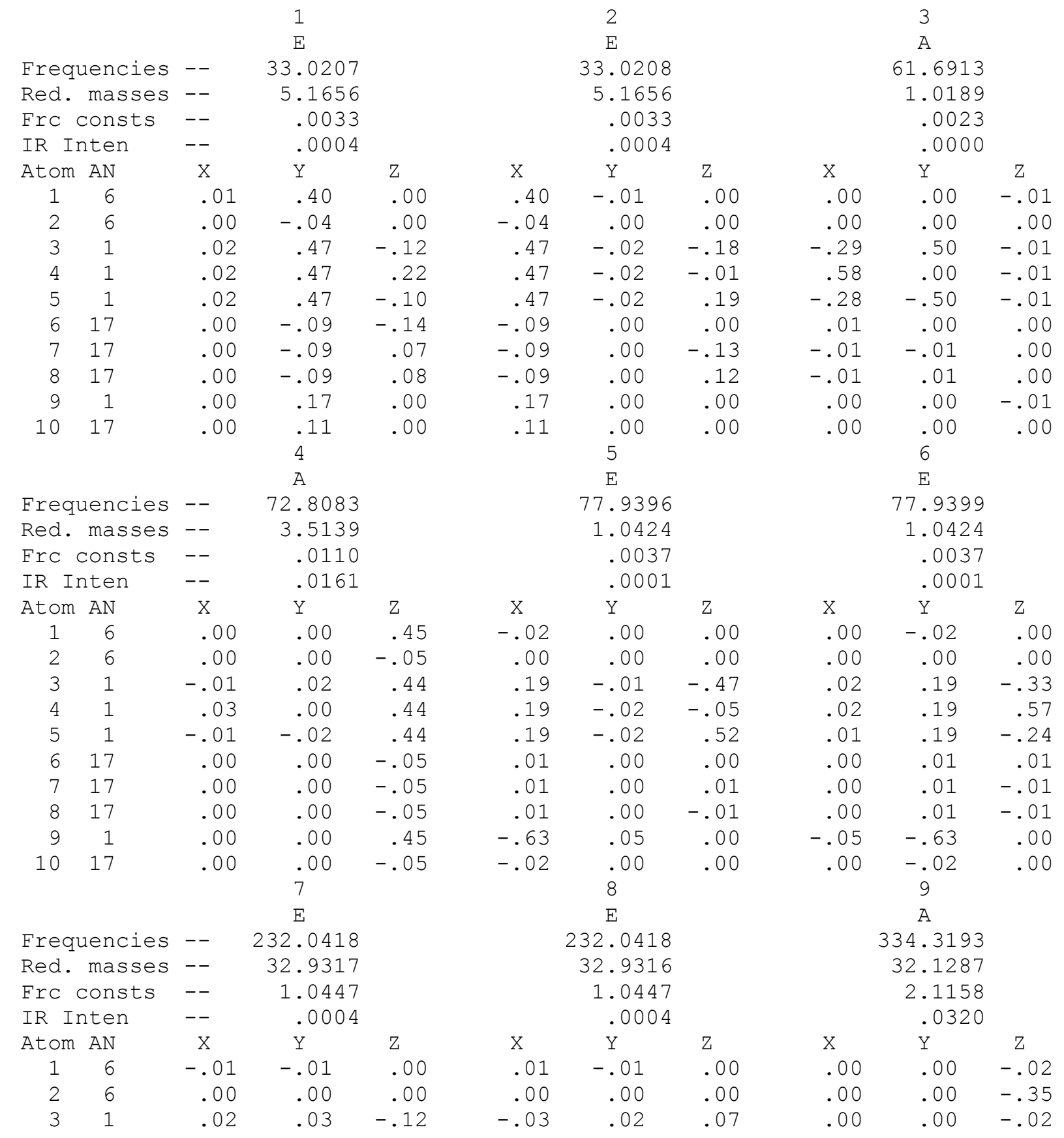




\begin{tabular}{|c|c|c|c|c|c|c|c|c|c|c|}
\hline 4 & 1 & .02 & .03 & .12 & -.03 & .02 & .07 & .00 & .00 & -.02 \\
\hline 5 & 1 & .02 & .03 & .01 & -.03 & .02 & -.14 & .00 & .00 & -.02 \\
\hline 6 & 17 & -.25 & .14 & .39 & .41 & .09 & .25 & .00 & .31 & .25 \\
\hline 7 & 17 & -.23 & -.42 & .02 & -.15 & .07 & -.46 & .27 & -.16 & .25 \\
\hline 8 & 17 & .24 & -.12 & -.41 & .15 & -.41 & .22 & -.27 & -.16 & .25 \\
\hline 9 & 1 & -.08 & -.14 & .00 & .14 & -.08 & .00 & .00 & .00 & -.02 \\
\hline \multirow[t]{3}{*}{10} & 17 & .25 & .41 & .00 & -.41 & .25 & .00 & .00 & .00 & -.63 \\
\hline & & & 10 & & & 11 & & & 12 & \\
\hline & & & $\mathrm{E}$ & & & $\mathrm{E}$ & & & A & \\
\hline \multicolumn{2}{|c|}{ Frequencies } & -- & 334.4744 & & & 334.474 & & & 475.8960 & \\
\hline \multicolumn{2}{|c|}{ Red. masses } & -- & 32.1044 & & & 32.104 & & & 34.9688 & \\
\hline \multirow{2}{*}{\multicolumn{2}{|c|}{$\begin{array}{l}\text { Frc consts } \\
\text { IR Inten }\end{array}$}} & -- & 2.1161 & & & 2.116 & & & 4.6661 & \\
\hline & & -- & .0576 & & & .057 & & & .0006 & \\
\hline \multicolumn{2}{|c|}{ Atom AN } & $\mathrm{x}$ & $\mathrm{Y}$ & Z & $\mathrm{x}$ & $\mathrm{Y}$ & Z & $\mathrm{x}$ & $\mathrm{Y}$ & Z \\
\hline 1 & 6 & .00 & .00 & .00 & .00 & .00 & .00 & .00 & .00 & .00 \\
\hline 2 & 6 & .31 & -.16 & .00 & .16 & .31 & .00 & .00 & .00 & .00 \\
\hline 3 & 1 & .00 & .00 & .00 & .00 & .00 & -.01 & .00 & .00 & .00 \\
\hline 4 & 1 & .00 & .00 & .00 & .00 & .00 & .01 & .00 & .00 & .00 \\
\hline 5 & 1 & .00 & .00 & .01 & .00 & .00 & .00 & .00 & .00 & .00 \\
\hline 6 & 17 & -.32 & -.24 & .14 & -.16 & .46 & -.28 & .00 & .47 & -.17 \\
\hline 7 & 17 & .44 & -.28 & -.31 & -.21 & -.30 & .02 & .41 & -.24 & -.17 \\
\hline 8 & 17 & .09 & .40 & .17 & .48 & .05 & .26 & -.41 & -.24 & -.17 \\
\hline 9 & 1 & -.01 & .00 & .00 & .00 & -.01 & .00 & .00 & .00 & .00 \\
\hline \multirow[t]{3}{*}{10} & 17 & -.32 & .16 & .00 & -.16 & -.32 & .00 & .00 & .00 & .5 \\
\hline & & & 13 & & & 14 & & & 15 & \\
\hline & & & $\mathrm{E}$ & & & $\mathrm{E}$ & & & A & \\
\hline \multicolumn{2}{|c|}{ Frequencies } & -- & 818.9948 & & & 318.994 & & & 820.1889 & \\
\hline \multirow{2}{*}{\multicolumn{2}{|c|}{$\begin{array}{l}\text { Red. masses } \\
\text { Frc consts }\end{array}$}} & -- & 13.0675 & & & 13.067 & & & 13.0686 & \\
\hline & & -- & 5.1642 & & & 5.1642 & & & 5.1797 & \\
\hline \multicolumn{2}{|c|}{ IR Inten } & -- & 147.4258 & & & -47.425 & & & 172.5277 & \\
\hline \multicolumn{2}{|c|}{ Atom AN } & $\mathrm{x}$ & $\mathrm{Y}$ & Z & $\mathrm{x}$ & $\mathrm{Y}$ & Z & $\mathrm{x}$ & $\mathrm{Y}$ & Z \\
\hline 1 & 6 & .00 & .00 & .00 & .00 & .00 & .00 & .00 & .00 & .0 \\
\hline 2 & 6 & -.02 & .98 & .00 & .98 & .02 & .00 & .00 & .00 & .98 \\
\hline 3 & 1 & .01 & -.01 & -.01 & .00 & .01 & -.01 & .00 & .00 & .01 \\
\hline 4 & 1 & .00 & .01 & .01 & -.02 & .00 & .00 & .00 & .01 & .01 \\
\hline 5 & 1 & -.01 & -.01 & -.01 & .00 & -.01 & .01 & .00 & .00 & .0 \\
\hline 6 & 17 & .00 & -.16 & .05 & -.04 & .00 & .00 & .00 & .05 & -.0 \\
\hline 7 & 17 & .06 & -.07 & -.02 & -.13 & .05 & .04 & .04 & -.02 & -.0 \\
\hline 8 & 17 & -.05 & -.07 & -.02 & -.13 & -.06 & -.04 & -.04 & -.02 & -.05 \\
\hline 9 & 1 & .00 & .01 & .00 & .01 & .00 & .00 & .00 & .00 & .0 \\
\hline \multirow[t]{3}{*}{10} & 17 & .00 & -.03 & .00 & -.03 & .00 & .00 & .00 & .00 & -.1 \\
\hline & & & 16 & & & 17 & & & 18 & \\
\hline & & & A & & & $E$ & & & $E$ & \\
\hline \multicolumn{2}{|c|}{ Frequencies } & -- & 1361.1383 & & & 362.163 & & & 362.1636 & \\
\hline \multirow{2}{*}{\multicolumn{2}{|c|}{$\begin{array}{l}\text { Red. masses } \\
\text { Frc consts }\end{array}$}} & -- & 1.1777 & & & 1.178 & & & 1.1788 & \\
\hline & & -- & 1.2856 & & & $1.288^{\circ}$ & & & 1.2887 & \\
\hline & -- & 22.6865 & & & 13.804 & & & 13.8041 & \\
\hline \multicolumn{2}{|c|}{$\begin{array}{l}\text { IR Inten } \\
\text { Atom AN }\end{array}$} & $\mathrm{X}$ & $\mathrm{Y}$ & Z & $\mathrm{x}$ & $\mathrm{Y}$ & Z & $\mathrm{x}$ & Y & Z \\
\hline 1 & 6 & .00 & .00 & -.12 & -.11 & -.06 & .00 & -.06 & .11 & .0 \\
\hline 2 & 6 & .00 & .00 & .00 & .00 & .00 & .00 & .00 & .00 & .06 \\
\hline 3 & 1 & -.19 & -.11 & .53 & .00 & -.04 & -.21 & .31 & -.53 & .01 \\
\hline 4 & 1 & .00 & .22 & .53 & .54 & .00 & .10 & .28 & .02 & -.1 \\
\hline 5 & 1 & .19 & -.11 & .53 & .24 & .45 & .12 & -.18 & -.28 & .1 \\
\hline 6 & 17 & .00 & .00 & .00 & .00 & .00 & .00 & .00 & .00 & .0 \\
\hline 7 & 17 & .00 & .00 & .00 & .00 & .00 & .00 & .00 & .00 & .0 \\
\hline 8 & 17 & .00 & .00 & .00 & .00 & .00 & .00 & .00 & .00 & .0 \\
\hline 9 & 1 & .00 & .00 & -.09 & .53 & .28 & .00 & .28 & -.53 & .0 \\
\hline
\end{tabular}




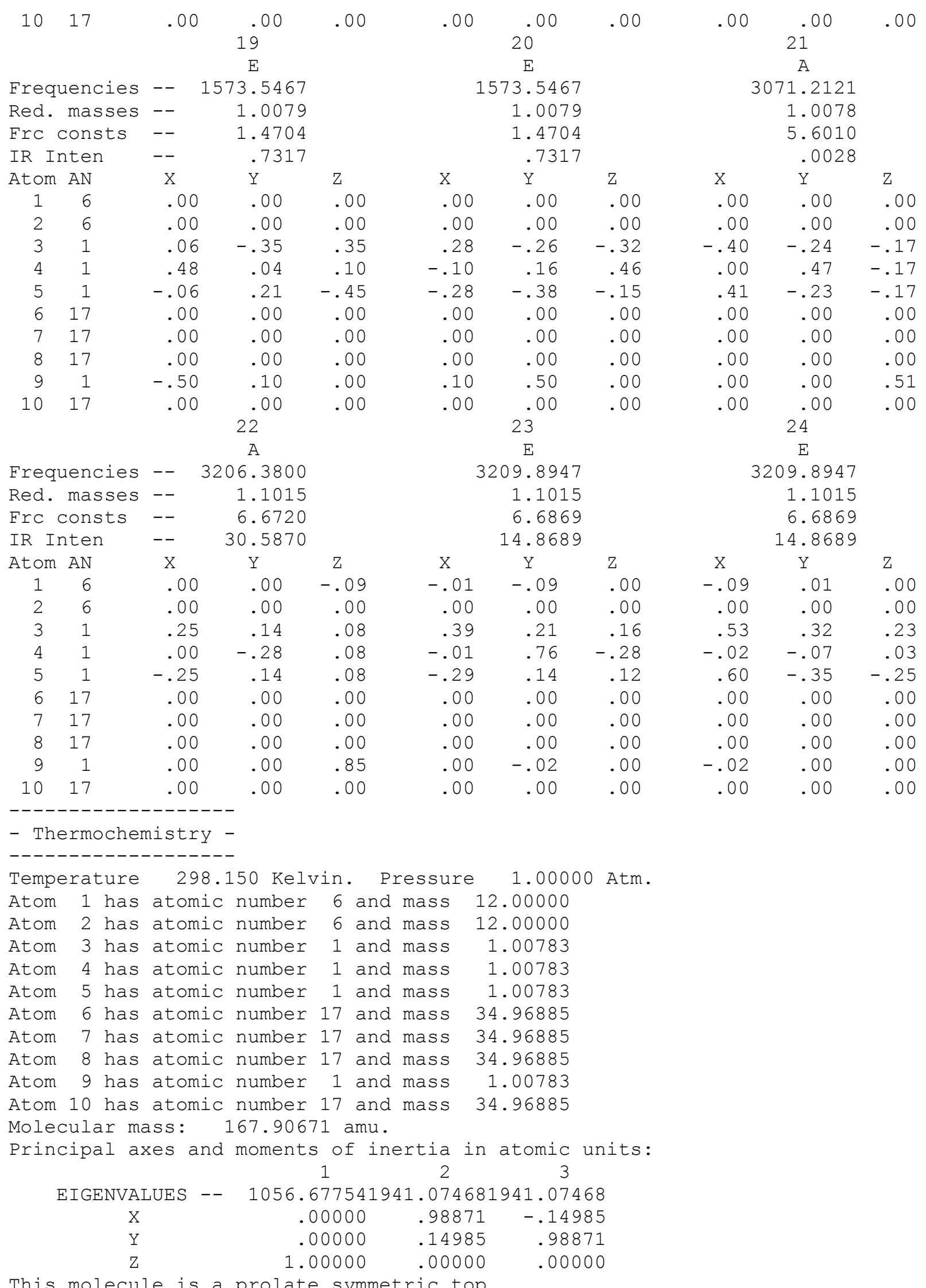




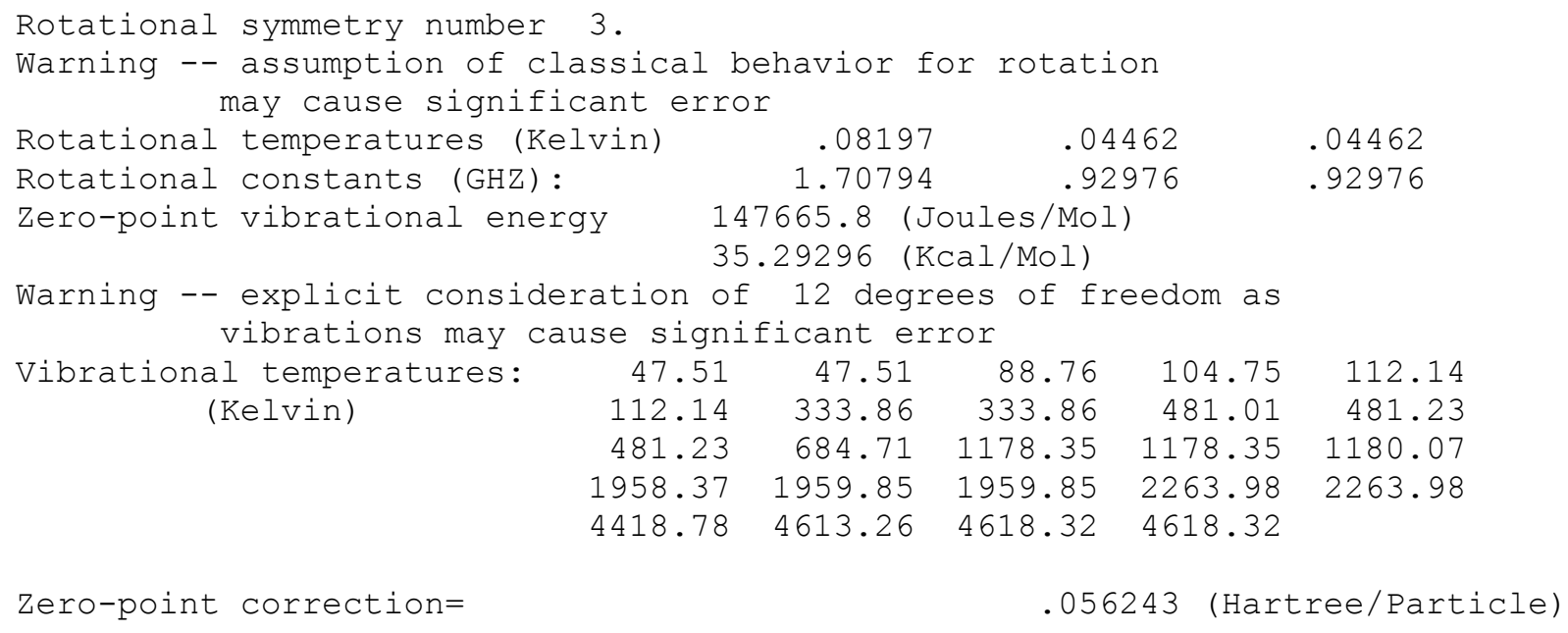


XXVII. Harmonic frequency analysis and zero-point energy for 1-monodeuterated methane-tetrachloromethane complex with the MP2/6$311++\mathrm{G}(\mathrm{d}, \mathrm{p})$ method:

Full mass-weighted force constant matrix:

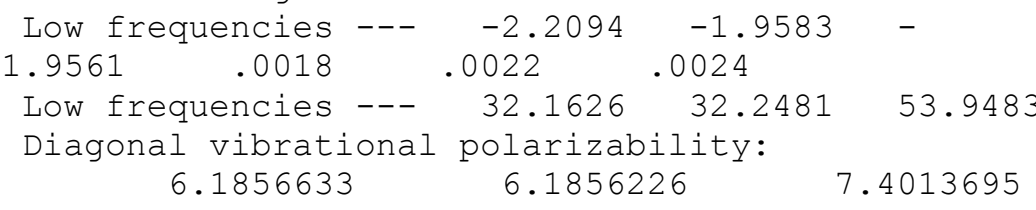

Harmonic frequencies ( $\left.\mathrm{cm}^{*} *-1\right)$, IR intensities (KM/Mole), Raman scattering activities ( $A^{* * 4 / A M U), ~ d e p o l a r i z a t i o n ~ r a t i o s ~ f o r ~ p l a n e ~ a n d ~ u n p o l a r i z e d ~}$ incident light, reduced masses (AMU), force constants (mDyne/A), and normal coordinates:

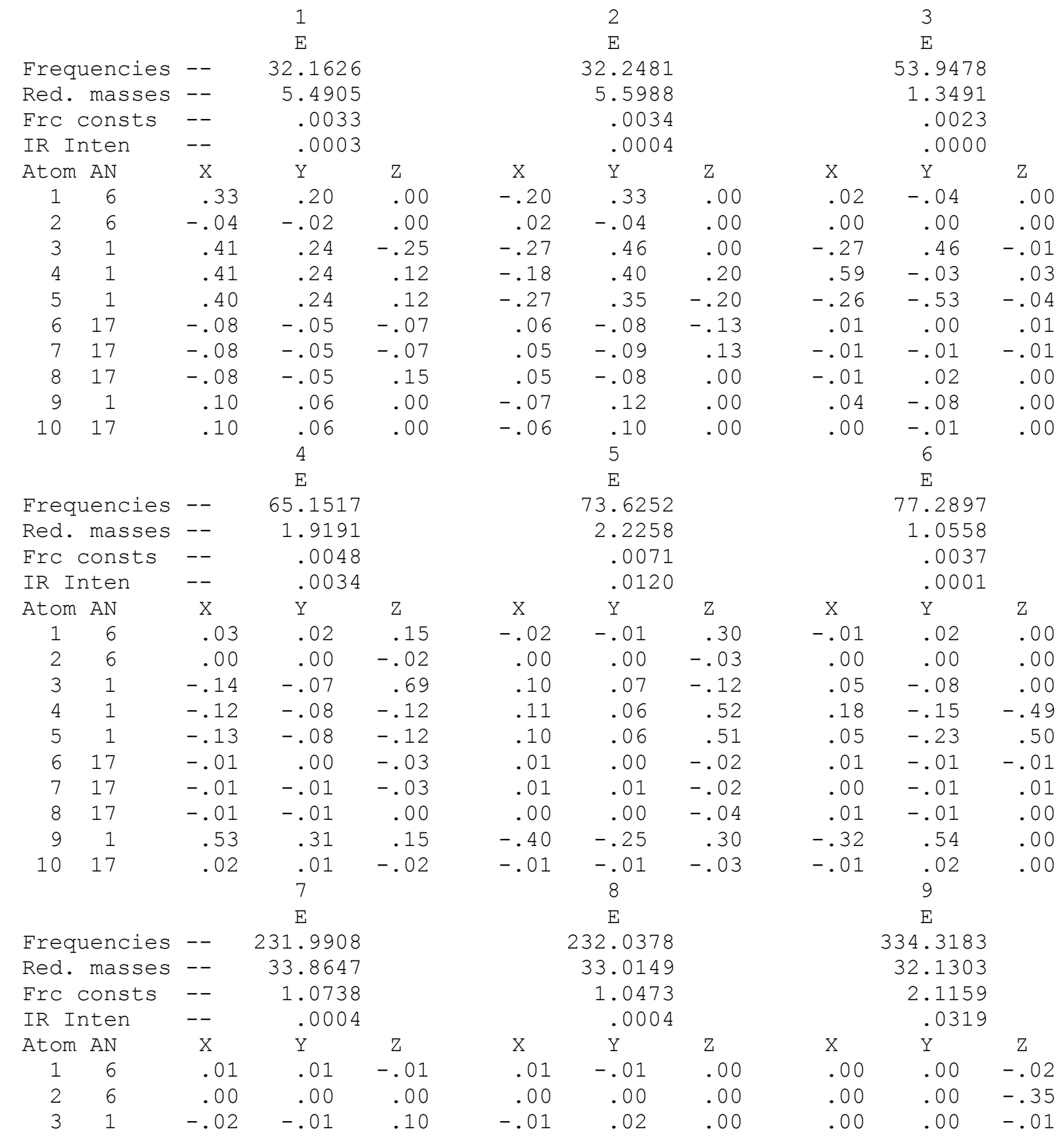




\begin{tabular}{|c|c|c|c|c|c|c|c|c|c|c|}
\hline 4 & 1 & -.02 & -.01 & -.06 & -.03 & .03 & .12 & .00 & .00 & -.02 \\
\hline 5 & 1 & -.02 & -.01 & -.06 & -.01 & .04 & -.12 & .00 & .00 & -.02 \\
\hline 6 & 17 & .42 & -.08 & -.24 & .24 & .14 & .40 & .00 & .31 & .25 \\
\hline 7 & 17 & .14 & .41 & -.23 & -.24 & -.13 & -.40 & .27 & -.16 & .25 \\
\hline 8 & 17 & -.14 & -.08 & .47 & .24 & -.42 & .00 & -.27 & -.16 & .25 \\
\hline 9 & 1 & .10 & .06 & -.01 & .08 & -.14 & .00 & .00 & .00 & -.0 \\
\hline \multirow[t]{3}{*}{10} & 17 & -.42 & -.25 & .00 & -.24 & .41 & .00 & .00 & .00 & -.63 \\
\hline & & & 10 & & & 11 & & & 12 & \\
\hline & & & $\mathrm{E}$ & & & $\mathrm{E}$ & & & A & \\
\hline \multicolumn{2}{|c|}{ Frequencies } & -- & \multicolumn{2}{|l|}{334.4741} & \multicolumn{3}{|c|}{334.4744} & \multicolumn{3}{|c|}{475.8960} \\
\hline \multicolumn{2}{|c|}{ Red. masses } & -- & 32.1070 & & \multicolumn{3}{|c|}{32.1043} & \multicolumn{3}{|c|}{34.9688} \\
\hline \multicolumn{2}{|c|}{ Frc consts } & -- & 2.1163 & & \multicolumn{3}{|c|}{2.1161} & \multicolumn{3}{|c|}{4.6661} \\
\hline \multicolumn{2}{|c|}{ IR Inten } & -- & .0575 & & \multicolumn{3}{|c|}{.0576} & \multicolumn{3}{|c|}{.0006} \\
\hline Atom & AN & $\mathrm{x}$ & $\mathrm{Y}$ & Z & $\mathrm{x}$ & $\mathrm{Y}$ & Z & $\mathrm{x}$ & $\mathrm{Y}$ & Z \\
\hline 1 & 6 & .00 & .00 & .00 & .00 & .00 & .00 & .00 & .00 & .00 \\
\hline 2 & 6 & .31 & .17 & .00 & .17 & -.31 & .00 & .00 & .00 & .00 \\
\hline 3 & 1 & .00 & .00 & -.01 & .00 & .00 & .00 & .00 & .00 & .0 \\
\hline 4 & 1 & .00 & .00 & .00 & .00 & .00 & -.01 & .00 & .00 & .00 \\
\hline 5 & 1 & .00 & .00 & .00 & .00 & .00 & .01 & .00 & .00 & .00 \\
\hline 6 & 17 & -.32 & .25 & -.15 & -.18 & -.46 & .27 & .00 & .47 & -.1 \\
\hline 7 & 17 & .08 & -.40 & -.16 & .48 & -.06 & -.27 & .41 & -.24 & -.1 \\
\hline 8 & 17 & .45 & .27 & .31 & -.19 & .31 & -.01 & -.41 & -.24 & -.1 \\
\hline 9 & 1 & .00 & .00 & .00 & .00 & .01 & .00 & .00 & .00 & .0 \\
\hline \multirow[t]{3}{*}{10} & 17 & -.31 & -.17 & .00 & -.17 & .31 & .00 & .00 & .00 & .5 \\
\hline & & & 13 & & & 14 & & & 15 & \\
\hline & & & $\mathrm{E}$ & & & $\mathrm{E}$ & & & $\mathrm{E}$ & \\
\hline Freq & uencies & -- & 818.9840 & & & 318.9905 & & & 820.1852 & \\
\hline Red. & masses & -- & 13.0631 & & & 13.065 & & & 13.0672 & \\
\hline Frc & consts & -- & 5.1624 & & & 5.1636 & & & 5.1791 & \\
\hline IR I & nten & -- & 147.3018 & & & 47.4562 & & & 172.6337 & \\
\hline Atom & AN & $\mathrm{x}$ & $\mathrm{Y}$ & Z & $\mathrm{X}$ & $\mathrm{Y}$ & Z & $\mathrm{X}$ & $\mathrm{Y}$ & Z \\
\hline 1 & 6 & .00 & .00 & .00 & .00 & .00 & .00 & .00 & .00 & .0 \\
\hline 2 & 6 & -.49 & .85 & .00 & .85 & .49 & .00 & .00 & .00 & .9 \\
\hline 3 & 1 & .01 & -.01 & .00 & .01 & .00 & -.01 & .00 & .00 & .0 \\
\hline 4 & 1 & .02 & .01 & .01 & -.02 & .00 & .00 & .00 & .01 & .0 \\
\hline 5 & 1 & -.02 & -.01 & -.01 & -.01 & -.02 & .00 & .01 & .00 & .01 \\
\hline 6 & 17 & .02 & -.14 & .04 & -.03 & -.08 & .02 & .00 & .05 & -.0 \\
\hline 7 & 17 & .11 & -.09 & -.04 & -.09 & .01 & .02 & .04 & -.02 & -.0 \\
\hline 8 & 17 & .02 & -.03 & .00 & -.14 & -.08 & -.05 & -.04 & -.02 & -.0 \\
\hline 9 & 1 & .00 & .00 & .00 & .01 & .01 & .00 & .00 & .00 &. \\
\hline 10 & 17 & .02 & -.03 & .00 & -.03 & -.02 & .00 & .00 & .00 & -.1 \\
\hline & & & 16 & & & 17 & & & 18 & \\
\hline & & & $\mathrm{E}$ & & & $\mathrm{E}$ & & & $E$ & \\
\hline Freq & uencies & -- & 1199.7028 & & & 201.0372 & & & 1356.1800 & \\
\hline Red. & masses & -- & 1.5081 & & & 1.5063 & & & 1.1643 & \\
\hline $\operatorname{Frc}$ & consts & -- & 1.2788 & & & 1.2802 & & & 1.2617 & \\
\hline IR I & nten & -- & 8.0917 & & & 14.829 & & & 15.1421 & \\
\hline Atom & AN & $\mathrm{x}$ & Y & Z & $\mathrm{x}$ & Y & Z & $\mathrm{x}$ & $\mathrm{Y}$ & Z \\
\hline 1 & 6 & .07 & -.13 & .00 & .04 & .02 & -.14 & -.09 & -.06 & -.0 \\
\hline 2 & 6 & .00 & .00 & .00 & .00 & .00 & .00 & .00 & .00 & .0 \\
\hline 3 & 1 & -.26 & .44 & .00 & -.15 & -.09 & .48 & -.09 & -.05 & -.0 \\
\hline 4 & 1 & -.51 & -.12 & -.03 & .06 & .20 & .40 & .50 & .07 & .2 \\
\hline 5 & 1 & .35 & .39 & .03 & .21 & -.05 & .40 & .30 & .40 & .2 \\
\hline 6 & 17 & .00 & .00 & .00 & .00 & .00 & .00 & .00 & .00 & .0 \\
\hline 7 & 17 & .00 & .00 & .00 & .00 & .00 & .00 & .00 & .00 & \\
\hline 8 & 17 & .00 & .00 & .00 & .00 & .00 & .00 & .00 & .00 &. \\
\hline 9 & 1 & -.21 & .35 & .00 & -.46 & -.27 & -.11 & .49 & .29 &.- \\
\hline
\end{tabular}




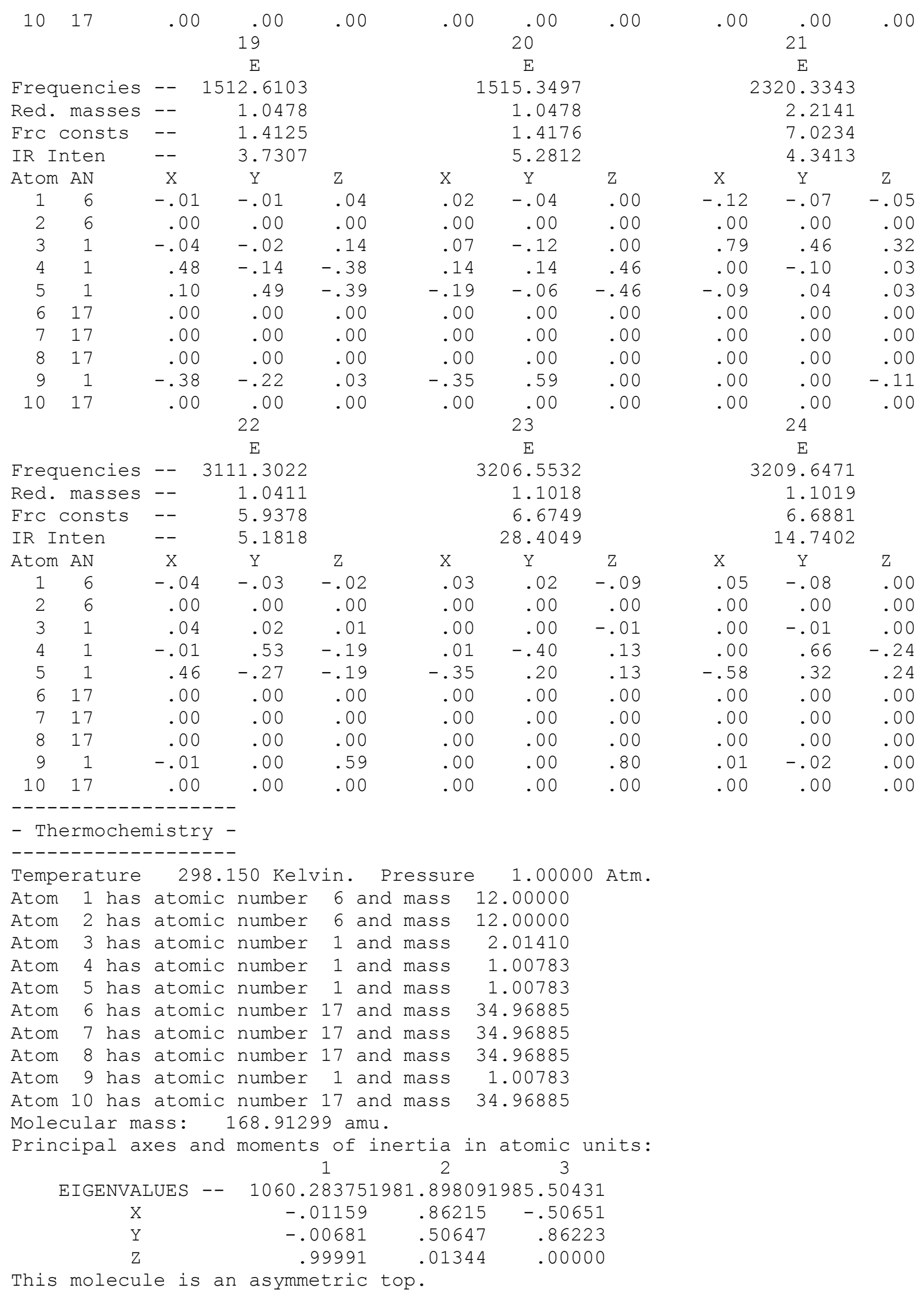




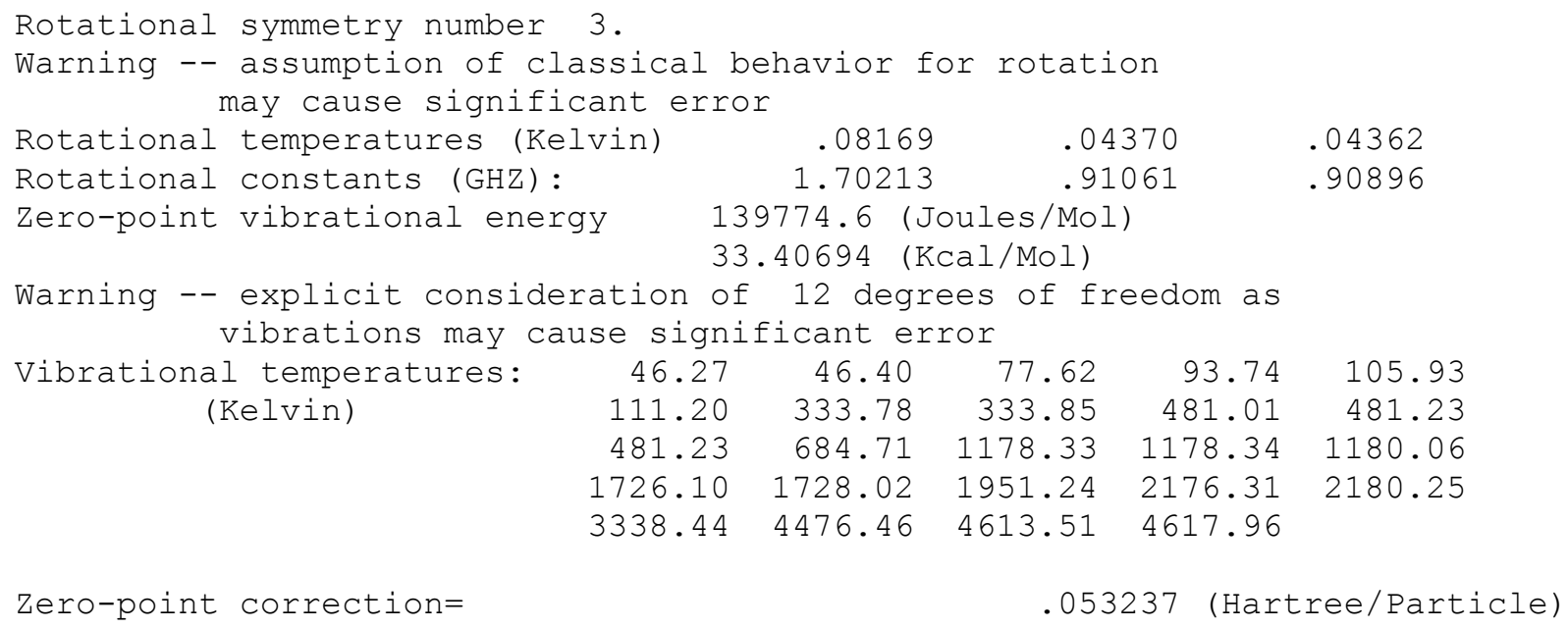


XXVIII. Harmonic frequency analysis and zero-point energy for 1,2-dideuterated methane-tetrachloromethane complex with the MP2/6$311++G(d, p)$ method:

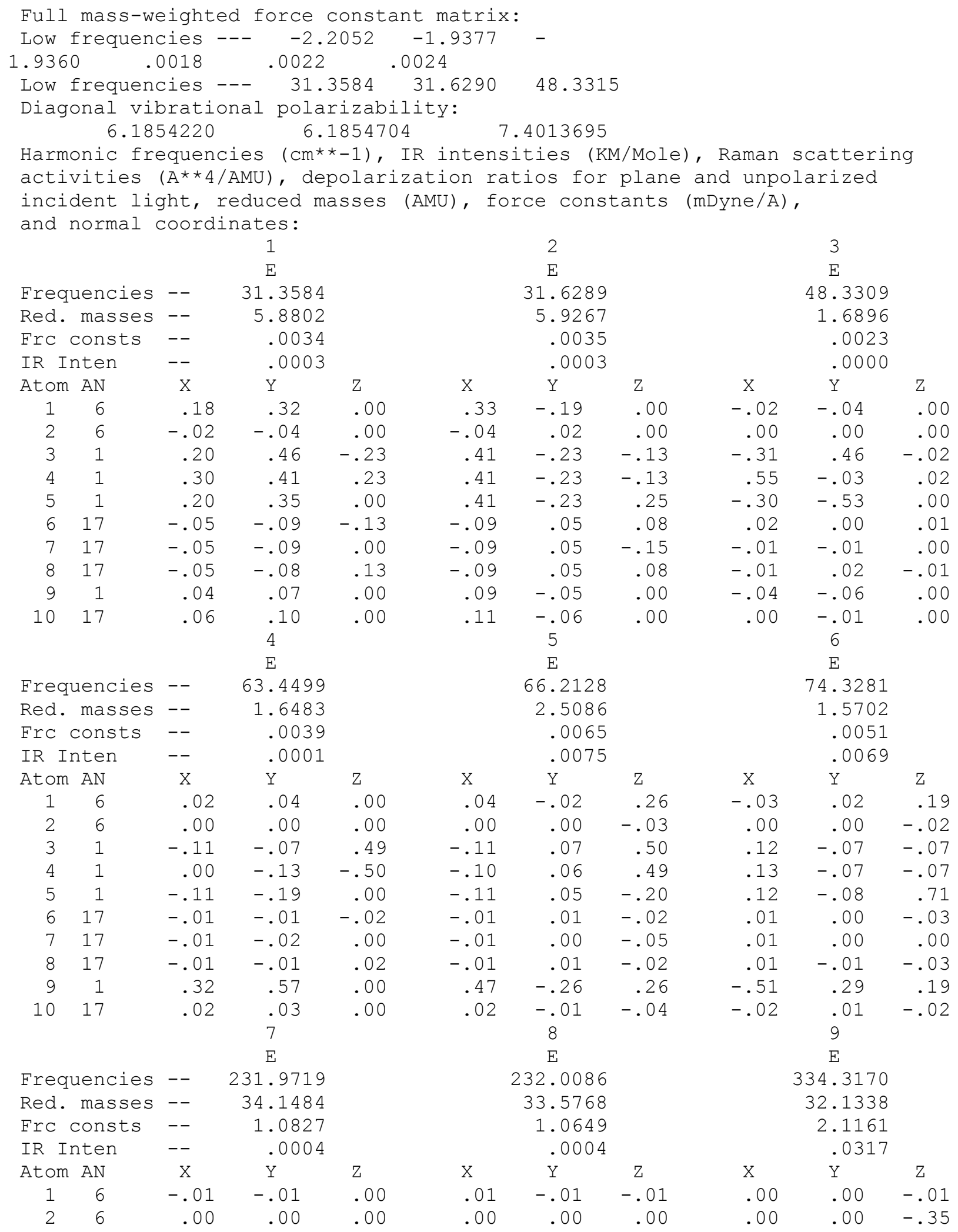




\begin{tabular}{|c|c|c|c|c|c|c|c|c|c|c|}
\hline 3 & 1 & .01 & .01 & -.08 & -.02 & .01 & .05 & .00 & .00 & -.01 \\
\hline 4 & 1 & .01 & .02 & .08 & -.02 & .01 & .05 & .00 & .00 & -.01 \\
\hline 5 & 1 & .01 & .02 & .00 & -.02 & .01 & -.12 & .00 & .00 & -.02 \\
\hline 6 & 17 & -.24 & .14 & .41 & .42 & .08 & .23 & .00 & .31 & .25 \\
\hline 7 & 17 & -.25 & -.42 & .00 & -.14 & .08 & -.47 & .27 & -.16 & .25 \\
\hline 8 & 17 & .25 & -.14 & -.41 & .14 & -.40 & .24 & -.27 & -.15 & .2 \\
\hline 9 & 1 & -.05 & -.09 & .00 & .12 & -.07 & -.01 & .00 & .00 & -.0 \\
\hline \multirow[t]{3}{*}{10} & 17 & .24 & .42 & .00 & -.42 & .24 & .00 & .00 & .00 & -.63 \\
\hline & & & 10 & & & 11 & & & 12 & \\
\hline & & & $\mathrm{E}$ & & & $\mathrm{E}$ & & & A & \\
\hline \multicolumn{2}{|c|}{ Frequencies } & -- & 334.4740 & & & 334.4742 & & & 475.8960 & \\
\hline \multicolumn{2}{|c|}{ Red. masses } & -- & 32.1077 & & & 32.1058 & & & 34.9688 & \\
\hline \multicolumn{2}{|c|}{ Frc consts } & -- & 2.1163 & & & 2.1162 & & & 4.6661 & \\
\hline \multicolumn{2}{|c|}{ IR Inten } & -- & .0575 & & & .0576 & & & .0006 & \\
\hline \multicolumn{2}{|c|}{ Atom AN } & $\mathrm{x}$ & $\mathrm{Y}$ & Z & $\mathrm{x}$ & $\mathrm{Y}$ & Z & $\mathrm{X}$ & $\mathrm{Y}$ & Z \\
\hline 1 & 6 & .00 & .00 & .00 & .00 & .00 & .00 & .00 & .00 & .00 \\
\hline 2 & 6 & .18 & .30 & .00 & .30 & -.18 & .00 & .00 & .00 & .00 \\
\hline 3 & 1 & .00 & .00 & .00 & .00 & .00 & .00 & .00 & .00 & .0 \\
\hline 4 & 1 & .00 & .00 & .00 & .00 & .00 & .00 & .00 & .00 & .0 \\
\hline 5 & 1 & .00 & .00 & .00 & .00 & .00 & .01 & .00 & .00 & .0 \\
\hline 6 & 17 & -.19 & .45 & -.27 & -.31 & -.27 & .16 & .00 & .47 & -.17 \\
\hline 7 & 17 & -.17 & -.32 & -.01 & .45 & -.25 & -.31 & .41 & -.24 & -.17 \\
\hline 8 & 17 & .48 & .08 & .27 & .06 & .40 & .15 & -.41 & -.24 & -.1 \\
\hline 9 & 1 & .00 & .00 & .00 & -.01 & .00 & .00 & .00 & .00 & .0 \\
\hline \multirow[t]{3}{*}{10} & 17 & -.18 & -.31 & .00 & -.31 & .18 & .00 & .00 & .00 & \\
\hline & & & 13 & & & 14 & & & 15 & \\
\hline & & & $E$ & & & $\mathrm{E}$ & & & $E$ & \\
\hline \multicolumn{2}{|c|}{ Frequencies } & -- & 818.9621 & & & 318.984 & & & 820.1790 & \\
\hline \multicolumn{2}{|c|}{ Red. masses } & -- & 13.0572 & & & 13.063 & & & 13.0653 & \\
\hline \multirow{2}{*}{\multicolumn{2}{|c|}{$\begin{array}{l}\text { Frc consts } \\
\text { IR Inten }\end{array}$}} & -- & 5.1597 & & & 5.162 & & & 5.1783 & \\
\hline & & -- & 147.1271 & & & 47.413 & & & 172.8055 & \\
\hline \multicolumn{2}{|c|}{ Atom AN } & $\mathrm{x}$ & $\mathrm{Y}$ & Z & $\mathrm{x}$ & $\mathrm{Y}$ & Z & $\mathrm{X}$ & $\mathrm{Y}$ & Z \\
\hline 1 & 6 & .00 & .00 & .00 & .00 & .00 & .00 & .00 & .00 & .00 \\
\hline 2 & 6 & .84 & -.49 & -.01 & .49 & .84 & .00 & .01 & .00 & .9 \\
\hline 3 & 1 & -.01 & .03 & -.01 & .01 & .00 & -.01 & .00 & -.01 & .0 \\
\hline 4 & 1 & -.03 & -.01 & -.01 & -.01 & .00 & .01 & .00 & .01 & .0 \\
\hline 5 & 1 & .01 & .00 & .01 & -.01 & -.03 & .00 & .01 & .00 & .0 \\
\hline 6 & 17 & -.03 & .08 & -.02 & -.02 & -.14 & .04 & .00 & .05 & -.0 \\
\hline 7 & 17 & -.14 & .08 & .05 & -.02 & -.03 & .00 & .04 & -.02 & -.0 \\
\hline 8 & 17 & -.09 & -.01 & -.02 & -.11 & -.09 & -.04 & -.04 & -.02 & -.0 \\
\hline 9 & 1 & .01 & .00 & .00 & .01 & .01 & .00 & .00 & .00 & .0 \\
\hline \multirow[t]{3}{*}{10} & 17 & -.03 & .02 & .00 & -.02 & -.03 & .00 & .00 & .00 & -.1 \\
\hline & & & 16 & & & 17 & & & 18 & \\
\hline & & & $\mathrm{E}$ & & & $E$ & & & $E$ & \\
\hline \multirow{2}{*}{\multicolumn{2}{|c|}{$\begin{array}{l}\text { Frequencies } \\
\text { Red. masses }\end{array}$}} & -- & 1061.0288 & & & 29.510 & & & 281.1615 & \\
\hline & & -- & 2.2751 & & & 1.7776 & & & 1.2683 & \\
\hline \multicolumn{2}{|c|}{ Frc consts } & -- & 1.5090 & & & 1.3362 & & & 1.2266 & \\
\hline \multicolumn{2}{|c|}{ IR Inten } & -- & 6.4380 & & & 13.5022 & & & 12.7614 & \\
\hline Atom & AN & $\mathrm{x}$ & Y & Z & $\mathrm{x}$ & $\mathrm{Y}$ & Z & $\mathrm{x}$ & $\mathrm{Y}$ & Z \\
\hline 1 & 6 & .12 & -.07 & .10 & -.09 & .05 & .15 & -.06 & -.11 & .0 \\
\hline 2 & 6 & -.01 & .00 & .00 & .00 & .00 & .00 & .00 & .00 & .0 \\
\hline 3 & 1 & -.12 & .61 & -.28 & .22 & -.13 & -.37 & -.14 & .12 & -.1 \\
\hline 4 & 1 & -.58 & -.21 & -.28 & .22 & -.13 & -.37 & .17 & -.06 & .1 \\
\hline 5 & 1 & .04 & -.02 & -.13 & -.25 & .14 & -.45 & .34 & .60 & .0 \\
\hline 6 & 17 & .00 & .00 & .00 & .00 & .00 & .00 & .00 & .00 & .0 \\
\hline 7 & 17 & .00 & .00 & .00 & .00 & .00 & .00 & .00 & .00 & .0 \\
\hline 8 & 17 & .00 & .00 & .00 & .00 & .00 & .00 & .00 & .00 & \\
\hline
\end{tabular}




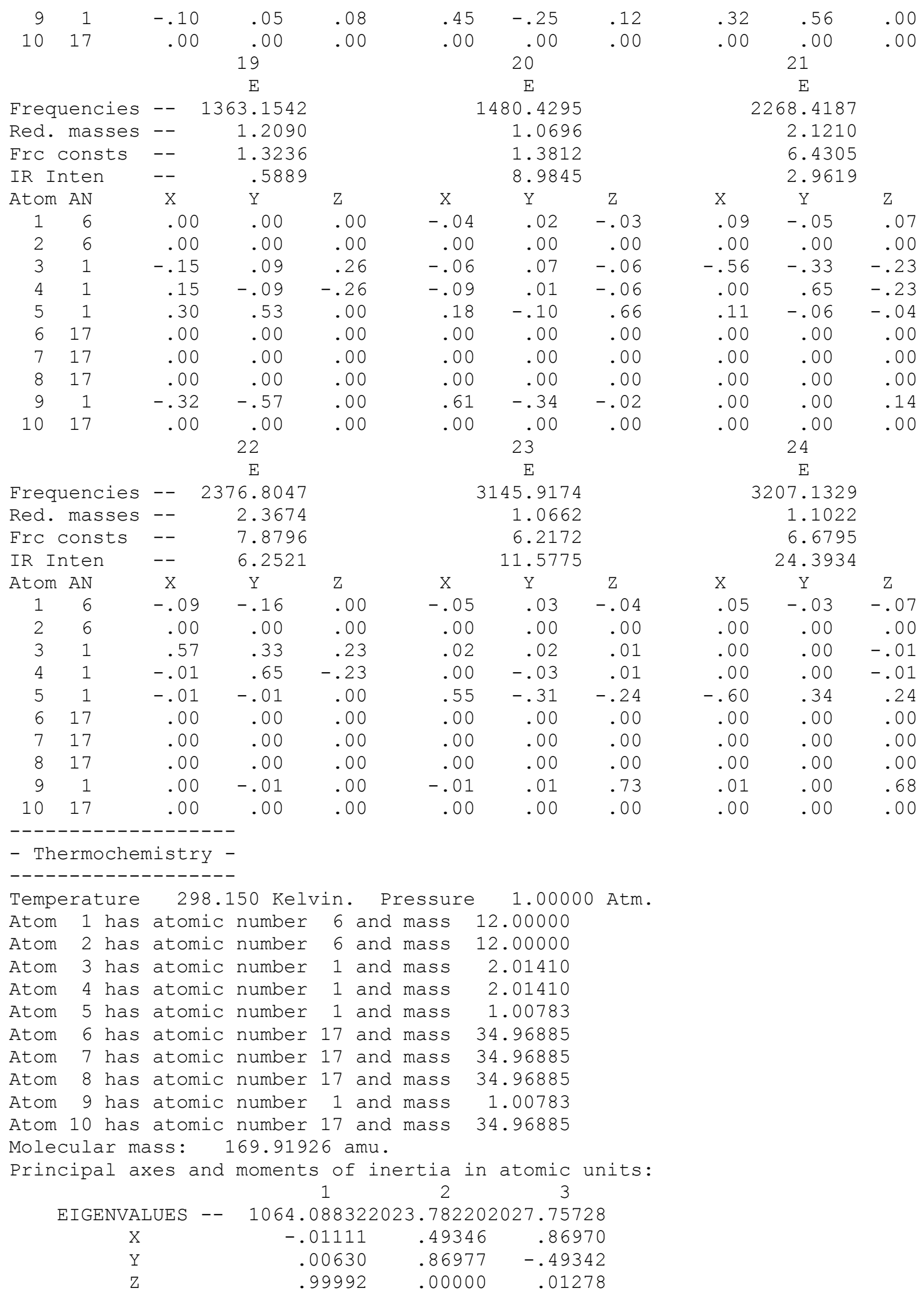


This molecule is an asymmetric top.

Rotational symmetry number 3 .

Warning -- assumption of classical behavior for rotation may cause significant error

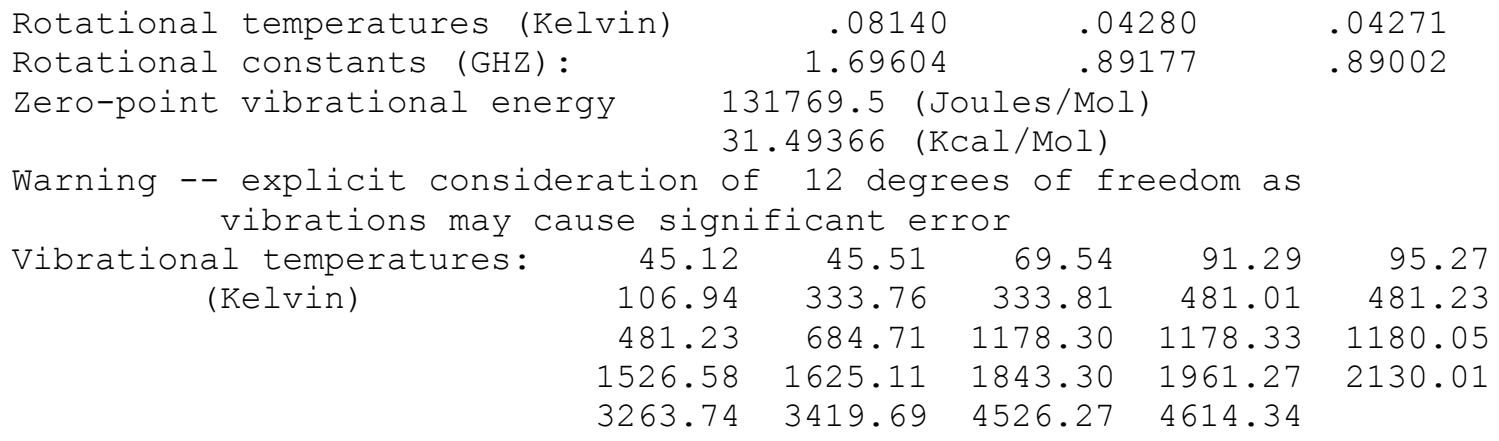

Zero-point correction=

.050188 (Hartree/Particle) 
XXIX. Harmonic frequency analysis and zero-point energy for 1,2,3-trideuterated methane-tetrachloromethane complex with the MP2/6$311++G(d, p)$ method:

Full mass-weighted force constant matrix:

$\begin{array}{rrrrl}\text { Low frequencies } & --- & -2.2004 & -1.9181 & - \\ 1.9170 \quad .0018 & .0022 & .0024 & \\ \text { Low frequencies }--- & 30.8742 & 30.8743 & 43.8938\end{array}$

Diagonal vibrational polarizability:

$$
6.1852603 \quad 6.1852600 \quad 7.4013695
$$

Harmonic frequencies $\left(\mathrm{cm}^{\star} \star-1\right)$, IR intensities (KM/Mole), Raman scattering activities $\left(A^{*} * 4 / \mathrm{AMU}\right)$, depolarization ratios for plane and unpolarized incident light, reduced masses (AMU), force constants (mDyne/A), and normal coordinates:

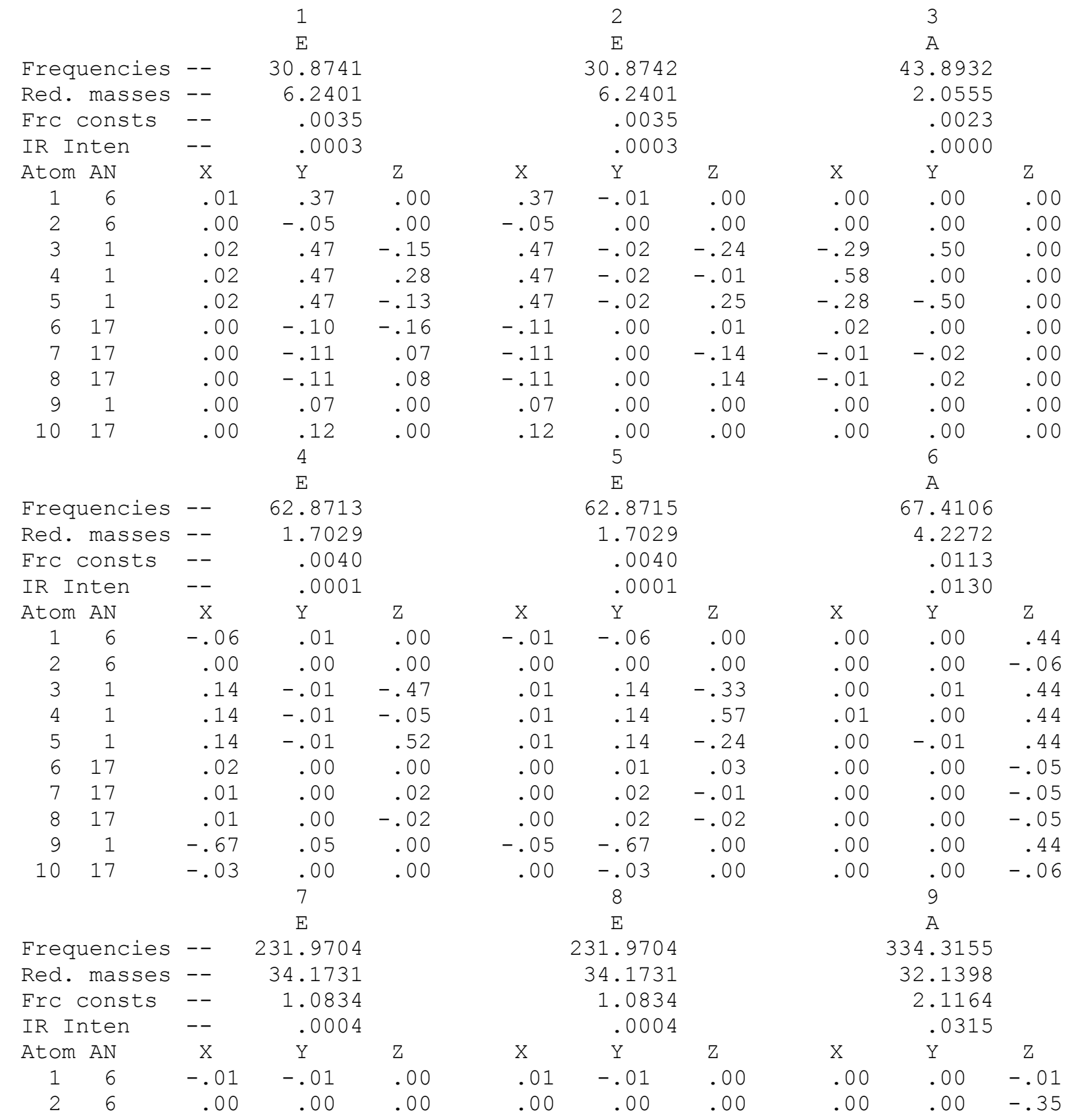




\begin{tabular}{|c|c|c|c|c|c|c|c|c|c|c|}
\hline 3 & 1 & .01 & .01 & -.08 & -.01 & .01 & .04 & .00 & .00 & -.01 \\
\hline 4 & 1 & .01 & .01 & .07 & -.02 & .01 & .05 & .00 & .00 & -.01 \\
\hline 5 & 1 & .01 & .02 & .01 & -.01 & .01 & -.09 & .00 & .00 & -.01 \\
\hline 6 & 17 & -.27 & .14 & .39 & .41 & .09 & .26 & .00 & .31 & .25 \\
\hline 7 & 17 & -.23 & -.43 & .03 & -.16 & .06 & -.47 & .27 & -.16 & .2 \\
\hline 8 & 17 & .24 & -.11 & -.42 & .16 & -.42 & .21 & -.27 & -.16 & .2 \\
\hline 9 & 1 & -.06 & -.09 & .00 & .09 & -.06 & .00 & .00 & .00 & -.01 \\
\hline \multirow[t]{3}{*}{10} & 17 & .27 & .41 & .00 & -.41 & .27 & .00 & .00 & .00 & -.63 \\
\hline & & & 10 & & & 11 & & & 12 & \\
\hline & & & $E$ & & & $E$ & & & A & \\
\hline & -- & 334.4739 & & \multicolumn{3}{|c|}{334.4739} & \multicolumn{3}{|c|}{$\begin{array}{c}\text { A } \\
475.8960\end{array}$} \\
\hline \multicolumn{2}{|c|}{$\begin{array}{l}\text { Frequencies } \\
\text { Red. masses }\end{array}$} & -- & 32.1075 & & \multicolumn{3}{|c|}{32.1075} & \multicolumn{3}{|c|}{34.9688} \\
\hline \multicolumn{2}{|c|}{ Frc consts } & -- & 2.1163 & & \multicolumn{3}{|c|}{2.1163} & \multicolumn{3}{|c|}{4.6661} \\
\hline \multicolumn{2}{|c|}{ IR Inten } & -- & .0575 & & \multicolumn{3}{|c|}{.0575} & \multicolumn{3}{|c|}{.0006} \\
\hline Atom & AN & $\mathrm{X}$ & $\mathrm{Y}$ & Z & $\mathrm{x}$ & $\mathrm{Y}$ & Z & $\mathrm{x}$ & $\mathrm{Y}$ & Z \\
\hline 1 & 6 & .00 & .00 & .00 & .00 & .00 & .00 & .00 & .00 & .0 \\
\hline 2 & 6 & .31 & -.16 & .00 & .16 & .31 & .00 & .00 & .00 & .0 \\
\hline 3 & 1 & .00 & .00 & .00 & .00 & .00 & .00 & .00 & .00 & .00 \\
\hline 4 & 1 & .00 & .00 & .00 & .00 & .00 & .00 & .00 & .00 & .00 \\
\hline 5 & 1 & .00 & .00 & .00 & .00 & .00 & .00 & .00 & .00 & \\
\hline 6 & 17 & -.32 & -.24 & .14 & -.17 & .46 & -.27 & .00 & .47 & -.17 \\
\hline 7 & 17 & .44 & -.27 & -.31 & -.20 & -.30 & .01 & .41 & -.24 & -.1 \\
\hline 8 & 17 & .09 & .40 & .17 & .48 & .05 & .26 & -.41 & -.24 & -.1 \\
\hline 9 & 1 & .00 & .00 & .00 & .00 & .00 & .00 & .00 & .00 & .00 \\
\hline \multirow[t]{3}{*}{10} & 17 & -.32 & .17 & .00 & -.17 & -.32 & .00 & .00 & .00 & \\
\hline & & & 13 & & \multicolumn{3}{|c|}{14} & & 15 & \\
\hline & & & $E$ & & & $E$ & & & A & \\
\hline Freq & uencies & -- & 818.9543 & & & 18.954 & & & 820.1693 & \\
\hline Red. & masses & -- & 13.0546 & & & 13.054 & & & 13.0630 & \\
\hline Frc & consts & -- & 5.1586 & & & 5.158 & & & 5.1773 & \\
\hline IR I & nten & -- & 147.1119 & & & 47.111 & & & 173.0769 & \\
\hline Atom & AN & $\mathrm{x}$ & Y & Z & $\mathrm{x}$ & $\mathrm{Y}$ & Z & $\mathrm{X}$ & $\mathrm{Y}$ & $\mathrm{Z}$ \\
\hline 1 & 6 & .00 & .00 & .00 & .00 & .00 & .00 & .00 & .00 & -.0 \\
\hline 2 & 6 & .01 & .98 & .00 & .98 & -.01 & .00 & .00 & .00 & .9 \\
\hline 3 & 1 & .02 & -.02 & -.01 & .00 & .02 & -.01 & -.01 & .00 & .0 \\
\hline 4 & 1 & .00 & .01 & .01 & -.03 & .00 & .00 & .00 & .01 & .0 \\
\hline 5 & 1 & -.02 & -.02 & -.01 & .00 & -.02 & .01 & .01 & .00 & $\cdot$ \\
\hline 6 & 17 & .00 & -.16 & .05 & -.04 & .00 & .00 & .00 & .05 & -.0 \\
\hline 7 & 17 & .05 & -.07 & -.02 & -.13 & .06 & .04 & .04 & -.02 & -.0 \\
\hline 8 & 17 & -.06 & -.07 & -.02 & -.13 & -.06 & -.04 & -.04 & -.02 & -.0 \\
\hline 9 & 1 & .00 & .01 & .00 & .01 & .00 & .00 & .00 & .00 & -.0 \\
\hline 10 & 17 & .00 & -.03 & .00 & -.03 & .00 & .00 & .00 & .00 & -.1 \\
\hline & & & 16 & & & 17 & & & 18 & \\
\hline & & & A & & & $E$ & & & $E$ & \\
\hline Freq & uencies & -- & 1036.9228 & & & 62.942 & & & 062.9420 & \\
\hline Red. & masses & -- & 2.5001 & & & 2.218 & & & 2.2184 & \\
\hline Frc & consts & -- & 1.5838 & & & 1.476 & & & 1.4768 & \\
\hline IR I & nten & -- & 12.7519 & & & 4.985 & & & 4.9853 & \\
\hline Atom & AN & $\mathrm{x}$ & $\mathrm{Y}$ & Z & $\mathrm{x}$ & $\mathrm{Y}$ & Z & $\mathrm{x}$ & $\mathrm{Y}$ & Z \\
\hline 1 & 6 & .00 & .00 & .23 & -.06 & -.15 & .00 & -.15 & .06 & .0 \\
\hline 2 & 6 & .00 & .00 & .01 & .00 & .01 & .00 & .01 & .00 & .0 \\
\hline 3 & 1 & .22 & .13 & -.49 & -.36 & .30 & .10 & .21 & -.58 & .0 \\
\hline 4 & 1 & .00 & -.25 & -.49 & .31 & -.18 & -.11 & .69 & .08 & .0 \\
\hline 5 & 1 & -.22 & .12 & -.49 & .39 & .64 & .01 & -.13 & .16 & -.1 \\
\hline 6 & 17 & .00 & .00 & .00 & .00 & .00 & .00 & .00 & .00 & \\
\hline 7 & 17 & .00 & .00 & .00 & .00 & .00 & .00 & .00 & .00 & . \\
\hline 8 & 17 & .00 & .00 & .00 & .00 & .00 & .00 & .00 & .00 & \\
\hline
\end{tabular}




\begin{tabular}{|c|c|c|c|c|c|c|c|c|c|c|}
\hline \multirow{4}{*}{$\begin{array}{r}9 \\
10\end{array}$} & 1 & .00 & .00 & .20 & .09 & .20 & .00 & .20 & -.09 & .00 \\
\hline & 17 & .00 & .00 & .00 & .00 & .00 & .00 & .00 & .00 & .00 \\
\hline & & & 19 & & \multicolumn{3}{|c|}{20} & \multicolumn{3}{|c|}{21} \\
\hline & & \multicolumn{3}{|c|}{$\mathrm{E}$} & \multicolumn{3}{|c|}{$E$} & \multicolumn{3}{|c|}{ A } \\
\hline \multicolumn{2}{|c|}{ Frequencies } & -- & \multicolumn{2}{|l|}{1331.0818} & \multicolumn{3}{|c|}{1331.0818} & \multicolumn{3}{|c|}{2219.7416} \\
\hline \multicolumn{2}{|c|}{ Red. masses } & -- & 1.2373 & & \multicolumn{3}{|c|}{1.2373} & \multicolumn{3}{|c|}{2.0584} \\
\hline \multicolumn{2}{|c|}{ Frc consts } & -- & 1.2917 & & \multicolumn{3}{|c|}{1.2917} & \multicolumn{3}{|c|}{5.9757} \\
\hline \multicolumn{2}{|c|}{ IR Inten } & -- & 7.2864 & & \multicolumn{3}{|c|}{7.2864} & \multicolumn{3}{|c|}{1.9633} \\
\hline Atom & $\mathrm{AN}$ & $\mathrm{x}$ & $\mathrm{Y}$ & Z & $\mathrm{x}$ & $\mathrm{Y}$ & Z & $\mathrm{x}$ & $\mathrm{Y}$ & Z \\
\hline 1 & 6 & -.07 & .01 & .00 & .01 & .07 & .00 & .00 & .00 & .08 \\
\hline 2 & 6 & .00 & .00 & .00 & .00 & .00 & .00 & .00 & .00 & .00 \\
\hline 3 & 1 & .01 & .06 & -.27 & -.06 & .04 & .19 & -.46 & -.27 & -.1 \\
\hline 4 & 1 & -.08 & -.01 & -.03 & .01 & -.05 & -.33 & .00 & .54 & -.1 \\
\hline 5 & 1 & .03 & -.05 & .30 & .05 & .05 & .14 & .47 & -.26 & -.1 \\
\hline 6 & 17 & .00 & .00 & .00 & .00 & .00 & .00 & .00 & .00 & .0 \\
\hline 7 & 17 & .00 & .00 & .00 & .00 & .00 & .00 & .00 & .00 & \\
\hline 8 & 17 & .00 & .00 & .00 & .00 & .00 & .00 & .00 & .00 & .0 \\
\hline 9 & 1 & .90 & -.08 & .00 & -.08 & -.90 & .00 & .00 & .00 & .1 \\
\hline \multirow[t]{3}{*}{10} & 17 & .00 & .00 & .00 & .00 & .00 & .00 & .00 & .00 & $\cdot v$ \\
\hline & & & 22 & & \multicolumn{3}{|c|}{23} & \multicolumn{3}{|c|}{24} \\
\hline & & \multicolumn{3}{|c|}{$E$} & \multicolumn{3}{|c|}{$\mathrm{E}$} & \multicolumn{3}{|c|}{ A } \\
\hline \multirow{2}{*}{\multicolumn{2}{|c|}{$\begin{array}{l}\text { Frequencies } \\
\text { Red. masses }\end{array}$}} & -- & 2376.7792 & & & 6.779 & & & 175.3691 & \\
\hline & masses & -- & 2.3681 & & & 2.368 & & & 1.0863 & \\
\hline FrC & consts & -- & 7.8817 & & & 7.881 & & & 6.4532 & \\
\hline IR I & hten & -- & 6.2163 & & & 6.216 & & & 23.4845 & \\
\hline Atom & AN & $\mathrm{X}$ & $\mathrm{Y}$ & Z & $\mathrm{X}$ & $\mathrm{Y}$ & Z & $\mathrm{x}$ & $\mathrm{Y}$ & Z \\
\hline 1 & 6 & .00 & -.19 & .00 & .19 & .00 & .00 & .00 & .00 & -.08 \\
\hline 2 & 6 & .00 & .00 & .00 & .00 & .00 & .00 & .00 & .00 & .0 \\
\hline 3 & 1 & .33 & .19 & .14 & -.56 & -.33 & -.23 & .02 & .01 & . \\
\hline 4 & 1 & -.01 & .76 & -.27 & .00 & .00 & .00 & .00 & -.02 & .0 \\
\hline 5 & 1 & -.33 & .18 & .13 & -.57 & .33 & .23 & -.02 & .01 & .0 \\
\hline 6 & 17 & .00 & .00 & .00 & .00 & .00 & .00 & .00 & .00 & .0 \\
\hline 7 & 17 & .00 & .00 & .00 & .00 & .00 & .00 & .00 & .00 & .0 \\
\hline 8 & 17 & .00 & .00 & .00 & .00 & .00 & .00 & .00 & .00 & .0 \\
\hline 9 & 1 & .00 & -.01 & .00 & .01 & .00 & .00 & .00 & .00 & 1.0 \\
\hline 10 & 17 & .00 & .00 & .00 & .00 & .00 & .00 & .00 & .00 & .0 \\
\hline
\end{tabular}

- Thermochemistry -

Temperature 298.150 Kelvin. Pressure 1.00000 Atm.

Atom 1 has atomic number 6 and mass 12.00000

Atom 2 has atomic number 6 and mass 12.00000

Atom 3 has atomic number 1 and mass 2.01410

Atom 4 has atomic number 1 and mass 2.01410

Atom 5 has atomic number 1 and mass 2.01410

Atom 6 has atomic number 17 and mass 34.96885

Atom 7 has atomic number 17 and mass 34.96885

Atom 8 has atomic number 17 and mass 34.96885

Atom 9 has atomic number 1 and mass 1.00783

Atom 10 has atomic number 17 and mass 34.96885

Molecular mass: 170.92554 amu.

Principal axes and moments of inertia in atomic units:

$\begin{array}{cccr}\text { EIGENVALUES -- } & 1068.063402067 .302332067 .30233 \\ \text { X } & .00000 & .17118 & .98524 \\ \text { Y } & .00000 & .98524 & -.17118 \\ \text { Z } & 1.00000 & .00000 & .00000\end{array}$


This molecule is a prolate symmetric top.

Rotational symmetry number 3 .

Warning -- assumption of classical behavior for rotation may cause significant error

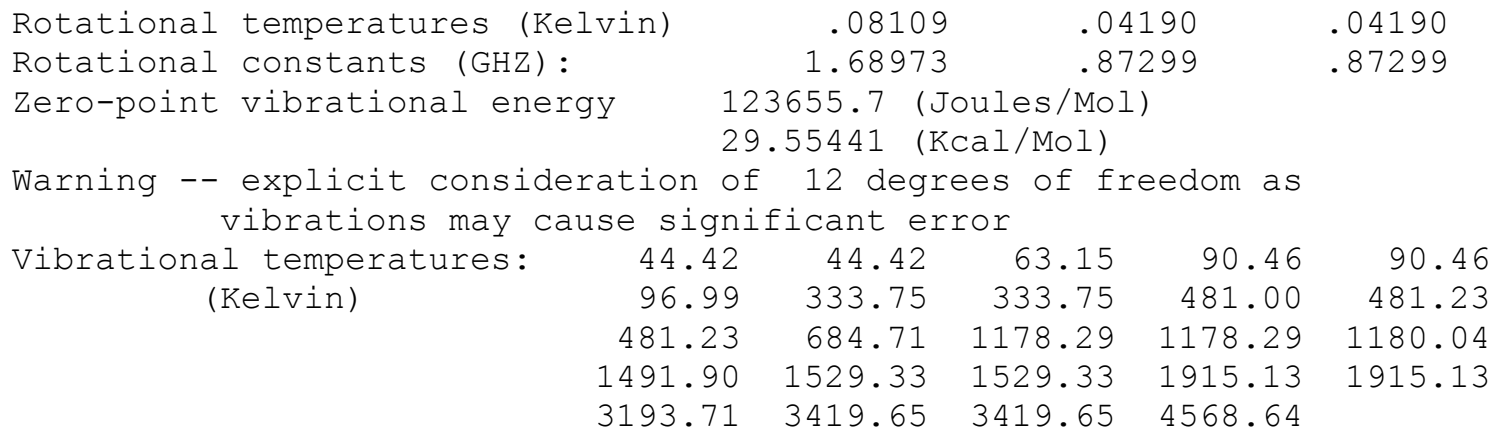

Zero-point correction=

.047098 (Hartree/Particle) 
XXX. Harmonic frequency analysis and zero-point energy for 1,2,3,4-tetradeuterated methane-tetrachloromethane complex with the MP2/6$311++G(d, p)$ method:

Full mass-weighted force constant matrix:

$\begin{array}{cccc}\text { Low frequencies --- } & -2.2004 & -1.8815 & - \\ 1.8804 \quad .0018 & .0021 & .0024 & \\ \text { Low frequencies --- } & 30.8629 & 30.8630 & 43.8937 \\ \text { Diagonal vibrational polarizability: } & \end{array}$

$$
6.1849967 \quad 6.1849965
$$

7.4013695

Harmonic frequencies ( $\mathrm{cm}^{\star \star}-1$ ), IR intensities (KM/Mole), Raman scattering activities (A**4/AMU), depolarization ratios for plane and unpolarized incident light, reduced masses (AMU), force constants (mDyne/A), and normal coordinates:

\begin{tabular}{|c|c|c|c|c|c|c|c|c|c|c|}
\hline & \multicolumn{3}{|c|}{1} & \multicolumn{3}{|c|}{2} & \multicolumn{3}{|c|}{3} \\
\hline & & \multicolumn{3}{|c|}{$\mathrm{E}$} & \multicolumn{3}{|c|}{$\mathrm{E}$} & \multicolumn{3}{|c|}{ A } \\
\hline Freq & uencies & -- & \multicolumn{2}{|l|}{30.8629} & \multicolumn{3}{|c|}{30.8629} & \multicolumn{3}{|c|}{43.8931} \\
\hline Red. & masses & -- & \multicolumn{2}{|l|}{6.3276} & \multicolumn{3}{|c|}{6.3276} & \multicolumn{3}{|c|}{2.0555} \\
\hline Frc & consts & -- & \multicolumn{2}{|l|}{.0036} & \multicolumn{3}{|c|}{.0036} & \multicolumn{3}{|c|}{.0023} \\
\hline IR I & nten & -- & \multicolumn{2}{|l|}{.0003} & \multicolumn{3}{|c|}{.0003} & \multicolumn{3}{|c|}{.0000} \\
\hline Atom & AN & X & Y & Z & $\mathrm{X}$ & Y & Z & $\mathrm{x}$ & Y & Z \\
\hline 1 & 6 & .01 & .37 & .00 & .37 & -.01 & .00 & .00 & .00 & .00 \\
\hline 2 & 6 & .00 & -.05 & .00 & -.05 & .00 & .00 & .00 & .00 & .00 \\
\hline 3 & 1 & .02 & .47 & -.15 & .47 & -.02 & -.24 & -.29 & .50 & .00 \\
\hline 4 & 1 & .02 & .47 & .28 & .47 & -.02 & -.01 & .58 & .00 & .00 \\
\hline 5 & 1 & .02 & .47 & -.13 & .47 & -.02 & .25 & -.28 & -.50 & .00 \\
\hline 6 & 17 & .00 & -.11 & -.16 & -.11 & .00 & .01 & .02 & .00 & .00 \\
\hline 7 & 17 & .00 & -.11 & .08 & -.11 & .00 & -.14 & -.01 & -.02 & .00 \\
\hline 8 & 17 & .00 & -.11 & .09 & -.11 & .00 & .14 & -.01 & .02 & .00 \\
\hline 9 & 1 & .00 & .07 & .00 & .07 & .00 & .00 & .00 & .00 & .00 \\
\hline \multirow[t]{3}{*}{10} & 17 & .00 & .13 & .00 & .13 & .00 & .00 & .00 & .00 & .00 \\
\hline & & \multirow{2}{*}{\multicolumn{3}{|c|}{$\begin{array}{l}4 \\
\mathrm{E}\end{array}$}} & \multirow{2}{*}{\multicolumn{3}{|c|}{5}} & \multicolumn{3}{|c|}{6} \\
\hline & & & & & & & & & A & \\
\hline Freq & uencies & -- & 56.1675 & & & 56.1677 & & & 65.8903 & \\
\hline Red. & masses & -- & 2.1796 & & & 2.1796 & & & 4.4692 & \\
\hline Frc & consts & -- & .0041 & & & .0041 & & & .0114 & \\
\hline IR I & nten & -- & .0001 & & & .0001 & & & .0126 & \\
\hline Atom & AN & X & $\mathrm{Y}$ & Z & $\mathrm{X}$ & $\mathrm{Y}$ & Z & $\mathrm{x}$ & $\mathrm{Y}$ & Z \\
\hline 1 & 6 & -.04 & .00 & .00 & .00 & -.04 & .00 & .00 & .00 & .44 \\
\hline 2 & 6 & .00 & .00 & .00 & .00 & .00 & .00 & .00 & .00 & -.06 \\
\hline 3 & 1 & .17 & -.01 & -.47 & .01 & .16 & -.33 & .00 & .01 & .44 \\
\hline 4 & 1 & .16 & -.01 & -.05 & .01 & .17 & .57 & .01 & .00 & .44 \\
\hline 5 & 1 & .17 & -.01 & .52 & .01 & .16 & -.24 & .00 & -.01 & .44 \\
\hline 6 & 17 & .02 & .00 & .00 & .00 & .02 & .03 & .00 & .00 & -.06 \\
\hline 7 & 17 & .02 & .00 & .03 & .00 & .02 & -.02 & .00 & .00 & -.06 \\
\hline 8 & 17 & .02 & .00 & -.03 & .00 & .02 & -.02 & .00 & .00 & -.06 \\
\hline 9 & 1 & -.64 & .05 & .00 & -.05 & -.64 & .00 & .00 & .00 & .44 \\
\hline 10 & 17 & -.04 & .00 & .00 & .00 & -.04 & .00 & .00 & .00 & -.06 \\
\hline & & & 7 & & & 8 & & & 9 & \\
\hline & & & E & & & E & & & A & \\
\hline Freq & uencies & -- & 231.9412 & & & 231.9413 & & & 334.3146 & \\
\hline Red. & masses & -- & 34.5006 & & & 34.5006 & & & 32.1425 & \\
\hline Frc & consts & -- & 1.0935 & & & 1.0935 & & & 2.1166 & \\
\hline IR I & nten & -- & .0004 & & & .0004 & & & .0316 & \\
\hline Atom & AN & X & Y & Z & $\mathrm{x}$ & $\mathrm{Y}$ & Z & $\mathrm{x}$ & $\mathrm{Y}$ & Z \\
\hline 1 & 6 & -.01 & -.01 & .00 & .01 & -.01 & .00 & .00 & .00 & -.01 \\
\hline 2 & 6 & .00 & .00 & .00 & .00 & .00 & .00 & .00 & .00 & -.35 \\
\hline
\end{tabular}




\begin{tabular}{|c|c|c|c|c|c|c|c|c|c|c|}
\hline 3 & 1 & .01 & .01 & -.06 & -.01 & .01 & .03 & .00 & .00 & -.01 \\
\hline 4 & 1 & .01 & .01 & .06 & -.01 & .01 & .04 & .00 & .00 & -.01 \\
\hline 5 & 1 & .01 & .01 & .01 & -.01 & .01 & -.07 & .00 & .00 & -.01 \\
\hline 6 & 17 & -.28 & .14 & .39 & .41 & .09 & .27 & .00 & .31 & .25 \\
\hline 7 & 17 & -.23 & -.43 & .04 & -.16 & .05 & -.47 & .27 & -.16 & .2 \\
\hline 8 & 17 & .24 & -.11 & -.43 & .16 & -.42 & .21 & -.27 & -.16 & .2 \\
\hline 9 & 1 & -.05 & -.07 & .00 & .07 & -.05 & .00 & .00 & .00 & -.01 \\
\hline \multirow[t]{3}{*}{10} & 17 & .28 & .41 & .00 & -.41 & .28 & .00 & .00 & .00 & -.63 \\
\hline & & & 10 & & & 11 & & & 12 & \\
\hline & & & $E$ & & & $E$ & & & A & \\
\hline & -- & 334.4738 & & \multicolumn{3}{|c|}{334.4738} & \multicolumn{3}{|c|}{$\begin{array}{c}\mathrm{A} \\
475.8960\end{array}$} \\
\hline \multicolumn{2}{|c|}{$\begin{array}{l}\text { Frequencies } \\
\text { Red. masses }\end{array}$} & -- & 32.1082 & & \multicolumn{3}{|c|}{32.1082} & \multicolumn{3}{|c|}{34.9688} \\
\hline \multicolumn{2}{|c|}{ Frc consts } & -- & 2.1164 & & \multicolumn{3}{|c|}{2.1164} & \multicolumn{3}{|c|}{4.6661} \\
\hline \multicolumn{2}{|c|}{ IR Inten } & -- & .0576 & & \multicolumn{3}{|c|}{.0576} & \multicolumn{3}{|c|}{.0006} \\
\hline Atom & AN & $\mathrm{X}$ & $\mathrm{Y}$ & Z & $\mathrm{x}$ & $\mathrm{Y}$ & Z & $\mathrm{x}$ & $\mathrm{Y}$ & Z \\
\hline 1 & 6 & .00 & .00 & .00 & .00 & .00 & .00 & .00 & .00 & .0 \\
\hline 2 & 6 & .31 & -.16 & .00 & .16 & .31 & .00 & .00 & .00 & \\
\hline 3 & 1 & .00 & .00 & .00 & .00 & .00 & .00 & .00 & .00 & .00 \\
\hline 4 & 1 & .00 & .00 & .00 & .00 & .00 & .00 & .00 & .00 & .00 \\
\hline 5 & 1 & .00 & .00 & .00 & .00 & .00 & .00 & .00 & .00 & \\
\hline 6 & 17 & -.32 & -.24 & .14 & -.17 & .46 & -.27 & .00 & .47 & -.1 \\
\hline 7 & 17 & .44 & -.27 & -.31 & -.20 & -.30 & .01 & .41 & -.24 & -.1 \\
\hline 8 & 17 & .09 & .40 & .17 & .48 & .05 & .26 & -.41 & -.24 & -.1 \\
\hline 9 & 1 & .00 & .00 & .00 & .00 & .00 & .00 & .00 & .00 & .0 \\
\hline \multirow[t]{3}{*}{10} & 17 & -.32 & .17 & .00 & -.17 & -.32 & .00 & .00 & .00 & \\
\hline & & & 13 & & \multicolumn{3}{|c|}{14} & & 15 & \\
\hline & & & $E$ & & & $E$ & & & A & \\
\hline Freq & uencies & -- & 818.9503 & & & 18.9503 & & & 820.1678 & \\
\hline Red. & masses & -- & 13.0534 & & & 13.0534 & & & 13.0620 & \\
\hline Frc & consts & -- & 5.1581 & & & 5.158 & & & 5.1768 & \\
\hline IR I & nten & -- & 147.2562 & & & 47.2562 & & & 173.1284 & \\
\hline Atom & AN & $\mathrm{x}$ & Y & Z & $\mathrm{x}$ & $\mathrm{Y}$ & Z & $\mathrm{X}$ & $\mathrm{Y}$ & $\mathrm{Z}$ \\
\hline 1 & 6 & .00 & .00 & .00 & .00 & .00 & .00 & .00 & .00 & -.0 \\
\hline 2 & 6 & .01 & .98 & .00 & .98 & -.01 & .00 & .00 & .00 &. \\
\hline 3 & 1 & .02 & -.02 & -.01 & .00 & .02 & -.01 & -.01 & .00 & .0 \\
\hline 4 & 1 & .00 & .01 & .02 & -.03 & .00 & .00 & .00 & .01 & .0 \\
\hline 5 & 1 & -.02 & -.02 & -.01 & .00 & -.02 & .01 & .01 & .00 & $\cdot$ \\
\hline 6 & 17 & .00 & -.16 & .05 & -.04 & .00 & .00 & .00 & .05 & -.0 \\
\hline 7 & 17 & .05 & -.07 & -.02 & -.13 & .06 & .04 & .04 & -.02 & -.0 \\
\hline 8 & 17 & -.06 & -.07 & -.02 & -.13 & -.06 & -.04 & -.04 & -.02 & -.0 \\
\hline 9 & 1 & .00 & .01 & .00 & .01 & .00 & .00 & .00 & .00 & -.0 \\
\hline 10 & 17 & .00 & -.03 & .00 & -.03 & .00 & .00 & .00 & .00 & -.1 \\
\hline & & & 16 & & & 17 & & & 18 & \\
\hline & & & A & & & $E$ & & & $E$ & \\
\hline Freq & uencies & -- & 1028.5092 & & & 29.526 & & & 029.5264 & \\
\hline Red. & masses & -- & 2.4685 & & & 2.469 & & & 2.4694 & \\
\hline Frc & consts & -- & 1.5385 & & & 1.542 & & & 1.5421 & \\
\hline IR I & nten & -- & 12.9023 & & & 8.581 & & & 8.5816 & \\
\hline Atom & AN & $\mathrm{x}$ & $\mathrm{Y}$ & Z & $\mathrm{x}$ & $\mathrm{Y}$ & Z & $\mathrm{x}$ & $\mathrm{Y}$ & Z \\
\hline 1 & 6 & .00 & .00 & .21 & .19 & .10 & .00 & -.10 & .19 & .0 \\
\hline 2 & 6 & .00 & .00 & .01 & .00 & .00 & .00 & .00 & .00 & .0 \\
\hline 3 & 1 & .21 & .13 & -.49 & .09 & .08 & .23 & .30 & -.51 & .0 \\
\hline 4 & 1 & .00 & -.25 & -.49 & -.53 & .05 & -.11 & .27 & .11 & -.2 \\
\hline 5 & 1 & -.22 & .12 & -.49 & -.19 & -.47 & -.13 & -.25 & -.23 & .2 \\
\hline 6 & 17 & .00 & .00 & .00 & .00 & .00 & .00 & .00 & .00 & \\
\hline 7 & 17 & .00 & .00 & .00 & .00 & .00 & .00 & .00 & .00 & \\
\hline 8 & 17 & .00 & .00 & .00 & .00 & .00 & .00 & .00 & .00 & \\
\hline
\end{tabular}




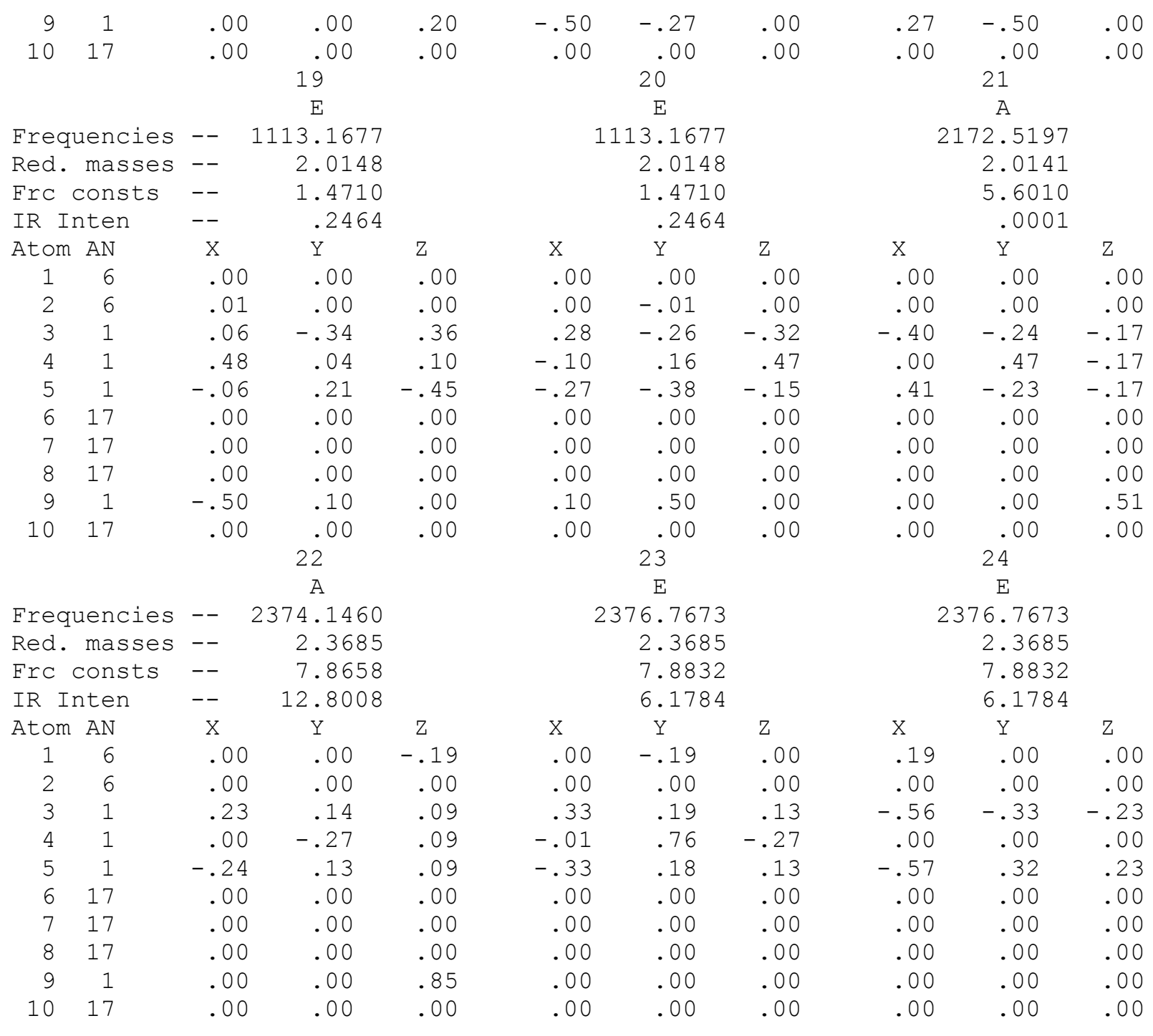

- Thermochemistry -

Temperature 298.150 Kelvin. Pressure 1.00000 Atm.

Atom 1 has atomic number 6 and mass 12.00000

Atom 2 has atomic number 6 and mass 12.00000

Atom 3 has atomic number 1 and mass 2.01410

Atom 4 has atomic number 1 and mass 2.01410

Atom 5 has atomic number 1 and mass 2.01410

Atom 6 has atomic number 17 and mass 34.96885

Atom 7 has atomic number 17 and mass 34.96885

Atom 8 has atomic number 17 and mass 34.96885

Atom 9 has atomic number 1 and mass 2.01410

Atom 10 has atomic number 17 and mass 34.96885

Molecular mass: $171.93182 \mathrm{amu}$.

Principal axes and moments of inertia in atomic units:

$\begin{array}{cccr}\text { EIGENVALUES -- } & 1068.063402148 .563942148 .56394 \\ \text { X } & .00000 & .26419 & .96447 \\ \text { Y } & .00000 & .96447 & -.26419 \\ \text { Z } & 1.00000 & .00000 & .00000\end{array}$


This molecule is a prolate symmetric top.

Rotational symmetry number 3 .

Warning -- assumption of classical behavior for rotation may cause significant error

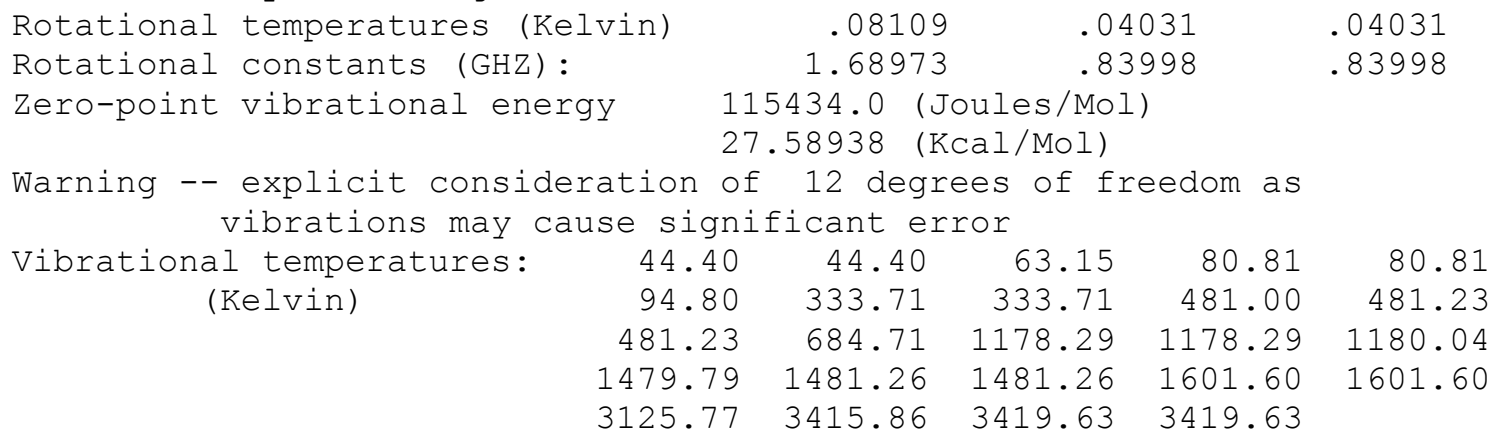

Zero-point correction=

.043966 (Hartree/Particle) 
XXXI. Calculation method for the multiple deuterium subsitutations as the following diagram, using non-deuterated methane as a reference. Because of Born-Oppenheimer approximation, the $\Delta \mathrm{E}_{\mathrm{elec}}$ in each complex is identical and isotopic fractionations between complexes can only be explained by differences in their zero point energies (ZPE).

Isotope effects for deuterations due to the formation of specific complexes:
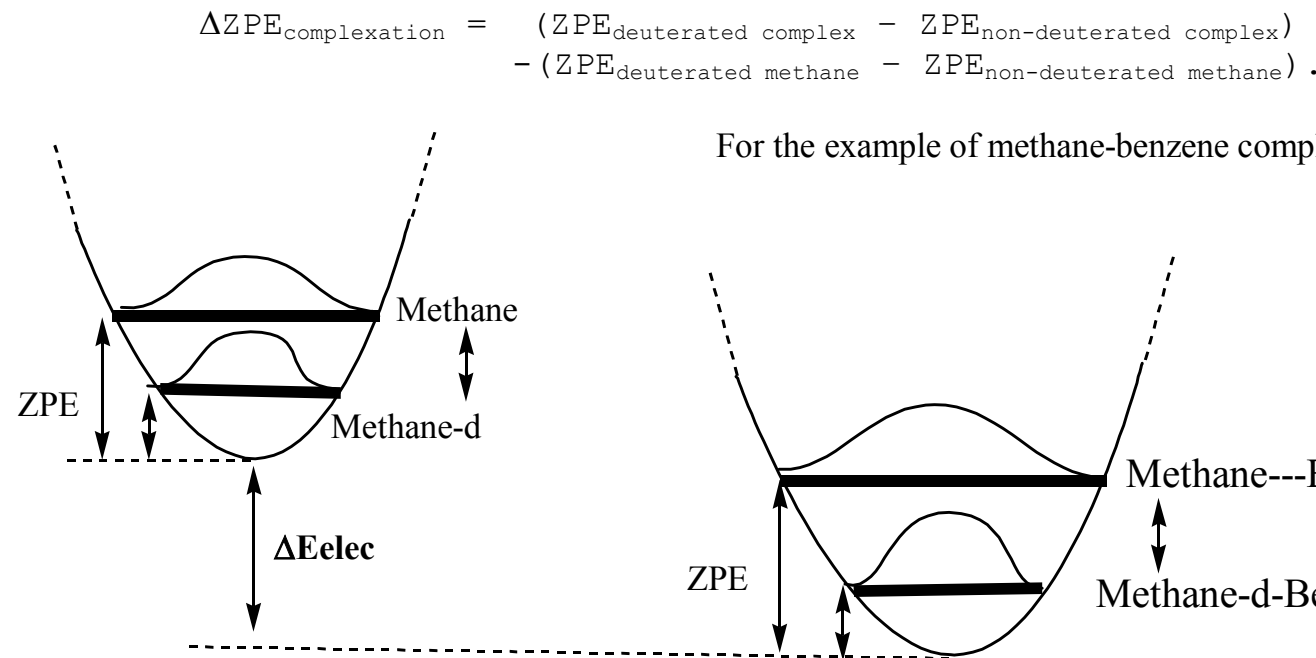

For the example of methane-benzene complex:

Isotope effects for the equilibrium between social isomers (assume that isotope effects for the interactions between methyl and phenyl are additive in the contact of methylresorcinarene):

In the case of $p$-xylene- $\mathrm{d}_{1}-\mathrm{CCl}_{4}-\mathbf{1}_{2}$ system:

$\Delta \mathrm{E}_{0}=(\Delta \mathrm{ZPE}$ methane-benzene $)-(\Delta \mathrm{ZPE}$ methane-CCl4 $)$;

for instance, if using $-\mathrm{d} 0$ and $-\mathrm{d} 1$ model, then:

$$
\begin{aligned}
& \Delta \mathrm{E}_{0}=(\Delta \mathrm{ZPE} \text { methane-benzene })-(\Delta \mathrm{ZPE} \text { methane-CCl4 }) \\
& =\left[\left(Z P E_{\text {methane-d1-benzene }}-Z P E_{\text {methane-benzene }}\right)-\left(\mathrm{ZPE}_{\text {methane-d1 }}-\mathrm{ZPE} \mathrm{E}_{\text {methane }}\right)\right] \\
& \text { - [(ZPE } \left.\left.\mathrm{Zmethane-d1-CCl4}_{-}-\mathrm{ZPE} \mathrm{E}_{\text {methane-CCl4 }}\right)-\left(\mathrm{ZPE}_{\text {methane-d1 }}-\mathrm{ZPE}_{\text {methane }}\right)\right] \\
& =\left(\mathrm{ZPE}_{\text {methane-d1-benzene }}+\mathrm{ZPE} \mathrm{E}_{\text {methane- } \mathrm{CCl} 14}\right) \\
& -\left(\mathrm{ZPE}_{\text {methane-benzene }}+\mathrm{ZPE} \mathrm{E}_{\text {methane-d1-CCl4 }}\right) \text {, }
\end{aligned}
$$

therefore, the result is identical to the direct calculation in the paper.

In the case of $p$-xylene $-\mathrm{d}_{2}-\mathrm{CCl}_{4}-\mathbf{1}_{2}$ system:

$\Delta \mathrm{E}_{0}=(\Delta \mathrm{ZPE}$ methane-benzene $) \times 2-(\Delta \mathrm{ZPE}$ methane-CCl4 $)$;

In the case of $p$-xylene $-\mathrm{d} 3-\mathrm{CCl}_{4}-\mathbf{1}_{2}$ system:

$\Delta \mathrm{E}_{0}=(\Delta \mathrm{ZPE}$ methane-benzene $) \times 3-(\Delta \mathrm{ZPE}$ methane-CCl4 $)$;

It is expected that the case involving $p$-xylene -d1 $_{1}$ best uses $\mathrm{d} 0 / \mathrm{d} 1$ models; while the cases involving $p$-xylene $e_{-\mathrm{d} 2}$ and $p$-xylene $-\mathrm{d} 3$ would best use $\mathrm{d} 0 / \mathrm{d} 2$, and $\mathrm{d} 0 / \mathrm{d} 3$, respectively.

Surprisingly, all $\mathrm{d} 0 / \mathrm{d} 1, \mathrm{~d} 0 / \mathrm{d} 2, \mathrm{~d} 0 / \mathrm{d} 3$ and even $\mathrm{d} 0 / \mathrm{d} 4$ give the similar results for $p$-xylene-d2 and $p$-xylene-d3 (see Table 2 in the paper). Our rationalization is that the EIEs are mainly due to methyl-resorcinarene interactions and not sensitive to methyl- $\mathrm{CCl}_{4}$ interactions. 
XXXII. Harmonic vibrational frequency change in the do and di models: NIST data:

Methane (gas phase): $1306 \mathrm{~cm}^{-1}$ (t2, deg. deform.); $1534 \mathrm{~cm}^{-1}$ (e, deg. deform.); $2917 \mathrm{~cm}^{-1}$ (a1, sym. str.); $3019 \mathrm{~cm}^{-1}$ (t2, deg. str.).

Deviation of $\sum$ hvi $=5 \%$ (ca./expl.)

Methane-d1 (gas phase) : $1155 \mathrm{~cm}^{-1}$ (e, CH3 rock); $1300 \mathrm{~cm}^{-1}$ (CH3 s-

deform.); $1471 \mathrm{~cm}^{-1}$ (e, CH3 d-deform.); $2200 \mathrm{~cm}^{-1}$ (C-D str.); $2945 \mathrm{~cm}^{-1}$ (e, CH3 s-str.); $3017 \mathrm{~cm}^{-1}$ (CH3 d-str.)

Deviation of $\sum$ hvi $=5 \%$ (ca./expl.)

\begin{tabular}{|c|c|c|c|c|c|}
\hline & & $-\mathrm{d} 0$ & $\sum_{-\mathrm{d} 1}$ & Change: & Persentages \\
\hline $\begin{array}{l}\text { Internal } \\
\text { (methane) }\end{array}$ & $\begin{array}{l}\text { Me-Ben } \\
\text { Me-CCl } 4\end{array}$ & $\begin{array}{l}19918.25 \\
19929.94\end{array}$ & $\begin{array}{l}18606.91 \\
18632.72\end{array}$ & -14.11 & $53 \%$ \\
\hline Intermolecular & $\begin{array}{l}\text { Me-Ben } \\
\text { Me-CCl } 4\end{array}$ & $\begin{array}{l}414.02 \\
356.42\end{array}$ & $\begin{array}{l}380.15 \\
334.42\end{array}$ & $-11 \cdot 88$ & $45 \%$ \\
\hline $\begin{array}{l}\text { Internal } \\
\text { (partner) }\end{array}$ & $\begin{array}{l}\text { Me-Ben } \\
\text { Me-CCl } 4\end{array}$ & $\begin{array}{r}43575.68 \\
4401.43\end{array}$ & $\begin{array}{r}43575.08 \\
4401.35\end{array}$ & -0.53 & $2 \%$ \\
\hline
\end{tabular}

For the social isomers involving p-xylene-dl,

Change $=\sum$ hvi for isomer deuterium/resorcinarene

- Khvi for isomer deuterium/tetrachloromethane

$\approx$ ( $\left(\right.$ hvi methane-di-Benzene $+\sum$ hvi methane-do-tetrachloromethane)

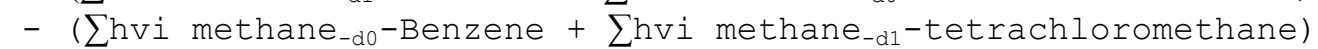

Total change $=-26.5 \mathrm{~cm}^{-1} ; \Delta \mathrm{E}_{0}=\Delta \mathrm{ZPE}=-13.3 \mathrm{~cm}^{-1}=-38 \mathrm{cal} / \mathrm{mol}$. 
XXXIII. Side-by-side vibrational frequencies $\left(\mathrm{cm}^{-1}\right)$ table (for clarity, the intramolecular vibrations of partners are omitted here, and vibrations with big isotope effects are marked.):

\begin{tabular}{|c|c|c|c|c|c|}
\hline & $\mathrm{CH}_{4}$-Benzene & $\mathrm{CH}_{4}-\mathrm{CCl}_{4}$ & & $\begin{array}{l}\mathrm{CDH}_{3}- \\
\text { Benzene }\end{array}$ & $\mathrm{CDH}_{3}-\mathrm{CCl}_{4}$ \\
\hline \multirow{8}{*}{ Symmetric } & Intramolecular & vibrational & frequencies & (methane, $\mathrm{cm}^{-1}$ ) & \\
\hline & 1353.776 & 1361.138 & & 1198.483 & 1199.703 \\
\hline & 1361.711 & 1362.164 & & 1198.485 & 1201.037 \\
\hline & 1361.711 & 1362.164 & & 1347.586 & 1356.18 \\
\hline & 1573.838 & 1573.547 & & 1511.891 & 1512.61 \\
\hline & 1573.838 & 1573.547 & & 1511.891 & 1515.35 \\
\hline & 3069.405 & 3071.212 & \multirow[t]{4}{*}{ C-D stretching } & 2332.434 & 2320.334 \\
\hline & 3200.819 & 3206.38 & & 3105.038 & 3111.302 \\
\hline \multirow[t]{7}{*}{ Degenerated } & 3200.819 & 3209.895 & & 3200.554 & 3206.553 \\
\hline & 3222.333 & 3209.895 & & 3200.554 & 3209.647 \\
\hline & \multicolumn{5}{|c|}{ Intermolecular vibrational frequencies $\left(\mathrm{cm}^{-1}\right)$} \\
\hline & 24.3372 & 33.0207 & & 24.3372 & 32.1626 \\
\hline & 43.0961 & 33.0208 & & 42.4316 & 32.2481 \\
\hline & 43.1041 & 61.6913 & & 42.4383 & 53.9478 \\
\hline & 84.5572 & 72.8083 & & 82.4998 & 65.1517 \\
\hline \multirow[b]{2}{*}{ Rocking } & 109.462 & 77.9396 & \multirow[b]{2}{*}{ Rocking } & 94.2194 & 73.6252 \\
\hline & 109.4688 & 77.9399 & & 94.2236 & 77.2897 \\
\hline
\end{tabular}

